\title{
Dimensionseffekte in
}

\section{Halbleiternanodrähten}

\author{
Dissertation
}

zur Erlangung des Doktorgrades

der Mathematisch-Naturwissenschaftlichen Fakultäten der Georg-August-Universität zu Göttingen

vorgelegt von

Daniel Stichtenoth

aus Göttingen

Göttingen 2008 
D 7

Referent:

Korreferent:

Tag der mündlichen Prüfung: 23.06.2008
Prof. Dr. Carsten Ronning

Prof. Dr. Cynthia A. Volkert 


\section{Inhaltsverzeichnis}

1 Einleitung 1

1.1 Motivation . . . . . . . . . . . . . . . . . 1

1.2 Zugänge zur Nanotechnologie . . . . . . . . . . . . . . . . 3

1.3 Nanodrähte als Bauelemente . . . . . . . . . . . . . . . . . 4

1.4 Forschungsgegenstand ................. 5

2 Experimentelles Vorgehen $\quad 9$

2.1 Synthese von Halbleiternanodrähten . . . . . . . . . . . . . . . . . 9

2.1.1 Der VLS-Mechanismus . . . . . . . . . . . . . . . . . . . . . 9

2.1.2 Abwandlungen des VLS-Mechanismus . . . . . . . . . 13

2.1.3 Nanodrahtherstellung in Göttingen . . . . . . . . . . . . . . 18

2.1.4 Nanodrahtherstellung in Kollaborationen . . . . . . . . . . 19

2.1.5 Zusammenfassung der Wachstumsprozesse . . . . . . . . . . 21

2.2 Modifikation von Halbleiternanodrähten . . . . . . . . . . . . . 23

2.2.1 Ionenimplantation . . . . . . . . . . . . . . . . . . 23

2.2.2 Dispersion von Nanodrähten . . . . . . . . . . . . 24

2.2.3 Elektrische Adressierung: Elektronenstrahllithographie . . . 25

2.3 Methoden zur Charakterisierung von Halbleiternanodrähten . . . . 25

2.3.1 Elektronenmikroskopische Verfahren . . . . . . . . . 25

2.3.2 Optische Spektroskopie . . . . . . . . . . . . . . . . 27

2.3.3 Elektrische Leitfähigkeit . . . . . . . . . . . . . . . 30

3 Optische Untersuchungen an ZnO-Nanodrähten 33

3.1 ZnO-Lumineszenz bei tiefer Temperatur . . . . . . . . . . . . . 34

3.2 Temperaturabhägigkeit der Lumineszenz . . . . . . . . . . . . . . . 37

3.3 Lumineszenz einzelner Nanodrähte . . . . . . . . . . . . . . . . . . 40

3.4 Einfluss der Oberfläche . . . . . . . . . . . . . . . . . . . . . . . . . 42

3.5 Quantumconfinement in ZnO-Nanodrähten . . . . . . . . . . . 45

3.6 Hochanregungsspektroskopie . . . . . . . . . . . . . . . 53

3.7 Donator-Akzeptor-Paarübergang in Zinkoxid . . . . . . . . . . . 57

3.8 Zusammenfassung . . . . . . . . . . . . . . . 66 
4.1 Strukturelle Veränderungen an ZnO-Nanodrähten durch Ionenimplantation . . . . . . . . . . . . . . . 70

4.1.1 Präparation der Proben . . . . . . . . . . . . . 70

4.1.2 Sputtereffekte durch Ionenbestrahlung . . . . . . . . . . 71

4.1.3 Strukturelle Veränderung des Kristallgitters durch Ionenimplantation . . . . . . . . . . . . . . . . 78

4.2 Strukturelle Veränderungen von GaAs-Nanodrähten durch Ionenbestrahlung ......................... 83

4.2.1 Präparation und strukturelle Eigenschaften der Proben . . . 83

4.2.2 Amorphisierung durch Ionenimplantation und Ausrichtung der Nanodrähte . . . . . . . . . . . . . . . 86

4.2.3 Rekristallisation durch Temperaturbehandlung . . . . . . . . 91

4.3 Zusammenfassung . . . . . . . . . . . . . . . . . . . . 92

$\begin{array}{llr}5 & \text { Leuchtzentren } & \mathbf{9 5}\end{array}$

5.1 Intra-3d-Lumineszenz im System in ZnS:Mn . . . . . . . . . . . . . 95

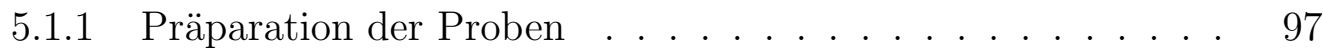

5.1.2 Optische Aktivierung des Mangans . . . . . . . . . . . . . . 99

5.1.3 Lebenszeitmessungen der Mangan-intra-3d-Lumineszenz . . 101

5.1.4 Abwandlung des Förstermodells für niedrigdimensionale Sys-

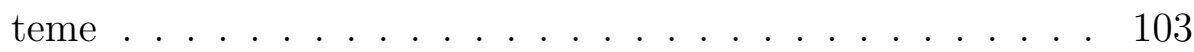

5.2 Intra-4f-Lumineszenz im System ZnO:RE . . . . . . . . . . . . . . 109

5.2.1 Aktivierung der intra-4f-Lumineszenz . . . . . . . . . . 110

5.2.2 Temperaturabhängigkeit der $\mathrm{Yb}^{3+}$-intra-4f-Lumineszenz . . . 115

5.2.3 Leistungsabhängigkeit der $\mathrm{Yb}^{3+}$-intra-4f-Lumineszenz . . . . 116

5.3 Zusammenfassung . . . . . . . . . . . . . . . . 117

6 Funktionalisierung von Nanodrähten als elektronische $\begin{array}{lr}\text { Bauelemente } & 121\end{array}$

6.1 p-Dotierung von GaAs-Nanodrähten . . . . . . . . . . . . . . . . . 121

6.1.1 Präparation der Proben . . . . . . . . . . . . . . . 121

6.1.2 Leitfähigkeitsmessungen an nichtimplantierten Galliumarsenid-Nanodrähten . . . . . . . . . . . . . . . 123

6.1.3 Leitfähigkeitsmessungen an Zink-implantierten Galliumarsenid-Nanodrähten . . . . . . . . . . . . . . . . . . 124

6.2 Feldeffekttransistoren basierend auf ZnO-Nanodrähten . . . . . . . 126

6.2.1 Herstellung der Feldeffekttransistoren . . . . . . . . . . . . . 126

6.2.2 Eigenschaften der Feldeffekttransistoren . . . . . . . . . . . 127

6.2.3 Einfluss des Nanodrahtdurchmessers . . . . . . . . . . . . 131

6.2.4 Passivierung der Nanodrahtoberfläche . . . . . . . . . . . 133 
6.2.5 Topgate-Feldeffekttransistor . . . . . . . . . . . . 135

6.3 Nanodraht-Leuchtdioden . . . . . . . . . . . . . . . . . . . . 138

6.3.1 Präparation der Proben . . . . . . . . . . . . . . 139

6.3.2 Leitfähigkeit und Elektrolumineszenz . . . . . . . . . . . . . 141

6.4 Zusammenfassung . . . . . . . . . . . . . . . . . . . 143

$\begin{array}{lll}7 & \text { Zusammenfassung und Ausblick } & 147\end{array}$

$\begin{array}{ll}\text { Literaturverzeichnis } & 151\end{array}$

$\begin{array}{ll}\text { Anhang } & 175\end{array}$ 



\section{Kapitel 1}

\section{Einleitung}

„Die Neigung der Menschen, kleine Dinge für wichtig zu halten, hat sehr viel Großes hervorgebracht."

-Georg Christoph Lichtenberg, Sudelbuch G (1742-1799)

\subsection{Motivation}

In den vergangenen Jahrzehnten war die fortschreitende Miniaturisierung von elektrischen Schaltungen die treibende Kraft des technischen Fortschrittes. Wie Gordon Moore es 1965 voraussagte, nahm die typische Größe von integrierten Schaltungen mit der Zeit exponentiell ab [Moo65]. Dies führte zu einer nie dagewesenen Integrationsdichte von nanoskaligen Bauelementen; mehr Komponenten auf weniger Raum bedeutet in der Mikroelektronik schnellere Datenverarbeitung, niedrigere Kosten und weniger Energieaufwand [Pee00] und somit auch nachhaltigerer Umgang mit Ressourcen.

Das große Interesse der Wissenschaften an Nanomaterialien ist insbesondere auf ihre außergewöhnlichen Eigenschaften zurückzuführen. Hier sind unter anderem Größenquantisierungseffekte und der dominierende Einfluss von Oberflächeneffekten zu nennen. Diese außergewöhnlichen Eigenschaften von Nanomaterialien, die Richard P. Feynman bereits 1959 in dem visionären Vortrag "There is plenty of room at the bottom" darstellte [Fey59], führten zu der Begründung eines neuen Forschungsgebietes: der Nanowissenschaft und -technologie. Die Nanowissenschaft ist ein stark interdisziplinäres Forschungsgebiet aus natur- 
wissenschaftlicher Grundlagenforschung in Physik und Chemie und angewandter Forschung in Ingenieurs- und Lebenswissenschaften. Der gemeinsame Schwerpunkt besteht in der Erforschung neuer Eigenschaften nanoskaliger Objekte sowie der Entwicklung von Methoden, um Nanomaterialien zu synthetisieren, zu charakterisieren und zu manipulieren [DB04, Sch06b, Sch06a]. Die Zielsetzungen bleibt dabei interdisziplinär.

Das Gebiet der Nanotechnologie, das oft als Schlüsseltechnologie des 21. Jahrhunderts bezeichnet wird, erweist sich als rapide wachsendes Forschungsgebiet. Während 1997 weltweit etwa 430 Mio. US\$ staatlicher Förderung in die Nanowissenschaften flossen, waren es 2005 bereits 4,1 Mrd. US\$ [Roc05].

Ursache dieses rapiden Wachstums ist vor allem der Zuspruch der Nanotechnologie, die Schlüsselprobleme des 21. Jahrhunderts zu adressieren. Dies sind allen voran die Abdeckung des weltweit zunehmenden Energiebedarfs, die Sicherung von Trinkwasser und die Verbesserung der medizinischen Versorgung [Sch06a]. Für jeden Bereich lassen sich Beispiele nennen, in denen Nanomaterialien zu entscheidenden Fortschritten führen: im Bereich der Abdeckung des wachsenden Energiebedarfs gibt es vor allem Ansätze, die zu effizienteren Bauelementen führen, wie zum Beispiel Konzepte hocheffizienter Solarzellen, die sich die große Oberfläche von Nanomaterialien zunutze machen [Law05, Ada04]. Zudem gibt es Beispiele aus der Energiespeicherung [Ari05] und Energieübertragung [Sch06a]. Im Bereich der Abdeckung der Trinkwasserversorgung können Nanomaterialien insbesondere Einsatz in Filteranlagen finden - ihre große Oberfläche bietet eine große aktive Fläche für eine effiziente Wasseraufbereitung [Sri04, Sha06]. Auch im Bereich der Medizin findet die Nanotechnologie breites Interesse - Schwerpunkte sind hier die Herstellung eines Lab-on-a-chip-Systems zur einfachen und kostengünstigen Diagnostik [Yag06] sowie die Funktionalisierung von Nanomaterialien zu Botenstoffen in einem Drugdelivery-System [Far06].

Schließlich führt die Grundlagenforschung an Nanomaterialien zu einer Reihe nie zuvor beobachteter Phänomene, wodurch sich neue Anwendungsgebiete erst erschlieBen - entsprechend wird die Nanotechnologie als enabling technology bezeichnet [Sch06a]. 


\subsection{Zugänge zur Nanotechnologie}

Zur Herstellung von Nanomaterialien werden zwei kontroverse Strategien verfolgt. Zum einen gibt es den so genannten top down-Ansatz, der vor allem in der Physik dominiert. Hierbei werden durch eine sequentielle Abfolge von Lithographie, Ätzprozess und Deposition Strukturen aus Festkörpern herausgearbeitet. Dieses Vorgehen ist in der Mikroelektronik ein etabliertes und erfolgreiches Verfahren. Um die herstellbare Strukturgröße weiter zu verkleinern, muss das Auflösungsvermögen der Lithographieanlagen verbessert werden; das bedeutet in der optischen Lithographie, entsprechend der Abbe'schen Auflösungsgrenze, die verwendete Wellenlänge zu reduzieren. In der aktuellen Prozessorgeneration von Intel werden Strukturgrößen von $45 \mathrm{~nm}$ verwendet, die mit Hilfe einer Mehrfachbelichtung (double patterning) bei einer Lichtwellenlänge von $193 \mathrm{~nm}$ erzeugt werden. Die für das kommende Jahr angekündigte Prozessorgeneration soll bereits auf $32 \mathrm{~nm}$-Strukturen basieren, die dann durch 193 nm-Immersionslithographie erreicht werden soll. Das Vorantreiben dieser Entwicklung geht mit einem exponentiellen Kostenanstieg für jede neue Strukturgröße einher, sodass die top down-Strategie nicht nur an ihre physikalische, sondern auch an ihre wirtschaftliche Grenze gerät [Mei01, Lie01].

Die zweite Strategie zur Herstellung von Nanostrukturen besteht in dem so genannten bottom up-Ansatz. Hierbei werden funktionale Strukturen gezielt aus atomaren bzw. molekularen Bausteinen zusammengesetzt, die zuvor in chemischen oder selbstorganisierten Verfahren hergestellt wurden - ähnlich wie in der Natur komplexe biologische Systeme aus Proteinen und anderen Makromolekülen zusammengesetzt werden. Dementsprechend wird dieser Ansatz bislang hauptsächlich in der Chemie und Biologie verfolgt. Die Herausforderungen dieser Strategie lassen sich sukzessiv in drei Schritte gliedern.

Die erste Herausforderung besteht darin, Bausteine mit einstellbarer Größe, Morphologie, chemischer Zusammensetzung und Struktur herzustellen. Zweitens die Charakterisierung der Strukturen, die Abwägung funktionaler Eigenschaften sowie eine gezielte Modifikation. Die dritte und zentrale Herausforderung dieser Strategie ist es, Methoden zur Anordnung der Bausteine in einer Architektur hoher Integrationsdichte und gewünschter Funktionalität zu finden [Wan04b]. Das bedeutet, dass Methoden zur gezielten Manipulation und Adressierung der Nanomaterialien 
entwickelt werden müssen.

\subsection{Nanodrähte als Bauelemente}

Ideale Bausteine für einen bottom up-Prozess sind einzelne Moleküle [Ree00, Joa00, Col99] und Quantenpunkte [Ali96, Kle97, Dev00], da diese - nach Atomen - die kleinsten Bausteine mit entsprechend großem Potential sind. Bereits 1925 erhielt der Göttinger Chemiker Richard Zsigmondy für seine bahnbrechende Erforschung der Eigenschaften von Kolloiden den Nobelpreis. In der Physik wurden Quantenpunkte im Wesentlichen in den letzten drei Jahrzehnten erforscht. Als problematisch stellte sich bei diesen Bausteinen der dritte und zentrale Schritt des Prozesses dar: die gezielte Anordnung der Bausteine zur Funktionalisierung [Wan04b].

Weitere aussichtsreiche Bausteine für einen bottom up-Prozess sind eindimensionale Strukturen [Lie01]. Ihr Potential besteht darin, dass sie das Modellsystem für thermischen und elektrischen Transport sowie für mechanische Eigenschaften niedrigdimensionaler Systeme sind [Xia03]. Darüber hinaus können eindimensionale Strukturen selbst durch gezielte Modifikation funktionelle Bauteile sein; so sind sie verbindendes und funktionelles Bauteil zugleich [Lie01, Col00]. Es wurde gezeigt, dass im Grunde jede Materialklasse auch in Form von Nanodrähten freistehend bzw. eingebettet synthetisiert werden kann. So finden sich unter anderem Berichte über metallische Nanodrähte [Mil06], organische Nanodrähte [Dou06] und Polymer-Nanodrähte [Li04]. Diese Arbeit befasst sich mit der Klasse der Halbleiternanodrähte. Hier wird meist noch zwischen Kohlenstoffnanoröhren (CNT) und Halbleiternanodrähten (SNW) unterschieden.

Seit ihrer Entdeckung durch Iijima 1991 [Iij91] werden CNT sehr intensiv erforscht. Dabei wurden hervorragende mechanische und elektrische Eigenschaften beobachtet [Col00]. In Abhängigkeit ihrer Helizität sind CNT entweder metallisch oder halbleitend [Odo98]. Ihre Funktionalisierung ist weitreichend fortgeschritten; hinsichtlich ihrer besonderen elektrischen Eigenschaften wurden beispielsweise Feldeffekttransistoren [Win02], logische Schaltungen [Der01, Bac00] und Feldemissionsdisplays [Lee00] aus CNT hergestellt. Aufgrund ihrer speziellen mechanischen Eigenschaften lassen sich CNT als Aktuatoren [Bau99] sowie als Cantileverspitzen in der Rastersondenmikroskopie [Dai96] einsetzen. Die kontrollierte Synthese von CNT 
hinsichtlich ihrer Helizität konnte bisher nicht realisiert werden; folglich ist eine nachträgliche Selektion notwendig [Col01].

Im Gegensatz zu den relativ gut erforschten CNT gibt es für SNW erst seit kurzer Zeit erfolgreiche Verfahren, diese kontrolliert und reproduzierbar, entsprechend der oben beschriebenen Anforderungen für Bausteine in einem bottom up-Prozess, zu synthetisieren [Dua00, Cui01a]. Halbleiternanodrähte lassen sich aus einer Vielzahl von Materialien in unterschiedlichen Morphologien synthetisieren, wobei die Wachstumsprozesse im Einzelnen nicht vollständig verstanden sind. Zu den Materialien gehören unter anderem bei den Elementhalbleitern Si [Wes97, Dew07] und Ge [Wu00], bei den binären Verbindungshalbleitern die Klasse der III-VHalbleiter (GaN, GaAs, GaP, InP, InAs [Joh06, Shi01]), der II-VI-Halbleiter $(Z n S, Z n S e, C d S, C d S e$ [Jia03, Sti05]) und der oxidischen Halbleiter ( $Z n O, M g O$, $\mathrm{SiO}_{2}$ [Che02, Wu01, Dua00]). Die wichtigsten Morpholgien der quasi eindimensionalen Systeme sind Drähte, Bänder, Röhren und Stäbe. Darüber hinaus gibt es eine Vielzahl zum Teil skurriler Formen, wie zum Beispiel Sägezahn- oder Spiralstrukturen [Ma03]. Die Kristallinität der Strukturen ist im Allgemeinen von hoher Güte. So vielfältig wie die Materialien und Morphologien sind auch die Verfahren zu ihrer Herstellung. Sie reichen von der chemischen Herstellung aus der Lösung über SolGel-Verfahren bis zur chemischen oder physikalischen Gasphasenabscheidung. Die Funktionalisierung der Bausteine ist ähnlich wie bei den CNT bereits relativ weit fortgeschritten. Elektrische Bauteile wie Dioden [Dua01a], Transistoren [Win02] und integrierte Schaltungen [Hua01b] wurden zum Teil durch Überkreuzen verschiedener Drähte, zum Teil aber auch durch gezielte Synthese von axialen [Gud02] und koaxialen [Lau02] Heterostrukturen erzeugt. Außerdem wurde gezeigt, dass sich Nanodrähte als Gas- und biologische Sensoren eignen [Cui01b, Pat06, Fan04].

\subsection{Forschungsgegenstand}

Diese Arbeit befasst sich mit der Charakterisierung, der gezielten Modifikation sowie der Funktionalisierung von Halbleiternanodrähten aus Zinksulfid, Zinkoxid und Galliumarsenid. Die Vorgehensweise orientiert sich an den drei zentralen Herausforderungen für die Funktionalisierung von Nanobausteinen in einem bottom upProzess (vgl. Abschnitt 1.2). Entsprechend gliedert sich auch diese Arbeit. 
Im zweiten Kapitel werden die verwendeten experimentellen Methoden und die Synthese von Halbleiternanodrähten in Göttingen sowie in verschiedenen Kollaborationen beschrieben und ein Wachstumsmodell vorgestellt. Dies adressiert die erste Herausforderung: die kontrollierte Synthese.

Die folgenden drei Kapitel befassen sich mit der Charakterisierung und der gezielten Modifikation der Strukturen und adressieren damit die zweite Herausforderung. Ein besonderer Schwerpunkt liegt hier auf dem Verständnis von Eigenschaften, durch die sich die Nanodrähte von Volumenmaterial unterscheiden.

Im dritten Kapitel werden systematische optische Untersuchungen an ZinkoxidNanodrähten vorgestellt. Hier werden insbesondere Effekte des vergrößerten Oberflächen-zu-Volumenverhältnis bei kleiner werdenden Strukturen sowie Effekte der Größenquantisierung und der Wellenleitereigenschaften hervorgehoben. Außerdem wird eine detaillierte optische Charakterisierung eines Donator-AkzeptorPaarüberganges vorgenommen, der nach dem Einbringen von Stickstoff in Zinkoxid durch Ionenimplantation und anschließender Temperaturbehandlung im Lumineszenzspektrum beobachtet werden konnte.

Das vierte Kapitel befasst sich mit der strukturellen Veränderung von Halbleiternanodrähten durch Ionenimplantation, die zur gezielten Veränderung von Eigenschaften der Nanodrähte verwendet wurde. Dabei treten insbesondere zwei Effekte auf: (1) die Veränderung der Morphologie durch das Abtragen von Oberflächenatomen und (2) die Schädigung des Kristallgitters bis hin zur Amorphisierung. Es werden Untersuchungen an zwei Systemen vorgestellt, bei denen zum einen die Veränderung der Morphologie und zum anderen die Gitterschädigung und deren Ausheilung durch eine geeignete Temperaturbehandlung im Vordergrund stehen. Im fünften Kapitel werden zwei Systeme vorgestellt, bei denen Leuchtzentren durch Ionenimplantation in Halbleiternanodrähte eingebracht und optisch aktiviert wurden: (1) das System ZnS:Mn und (2) das System ZnO:RE. An dem ersten System werden detaillierte Untersuchungen zur Lebenszeit der Mangan-intra-3d Lumineszenz als Funktion der Mangankonzentration sowie der Strukturgröße durchgeführt und ein entsprechendes Modell aufgestellt. An dem zweiten System werden die optischen Untersuchungen insbesondere hinsichtlich der lokalen Umgebung der Seltenen Erden diskutiert.

Das sechste Kapitel befasst sich mit Konzepten zur elektrischen Modifikation und 
Funktionalisierung von Halbleiternanodrähten und adressiert damit die dritte Herausforderung eines bottom up-Prozesses. Es werden zunächst Untersuchungen zur pDotierung von Galliumarsenid-Nanodrähten mittels Ionenimplantation vorgestellt. Der zweite Abschnitt des Kapitels befasst sich mit Zinkoxid-Nanodrähten, die zu Feldeffekttransistoren prozessiert wurden. Hier wird der Einfluss der Nanodrahtoberfläche sowie der Geometrie der Basis auf die Kenngrößen des Transistors untersucht und diskutiert. Im letzten Abschnitt dieses Kapitels wird eine Methode vorgestellt, mit der Zinkoxid-Nanodrähte zu UV-Leuchtdioden prozessieren wurden.

Im letzten Kapitel wird eine kurze Zusammenfassung dieser Arbeit sowie ein Ausblick gegeben. 


\section{Kapitel 2}

\section{Experimente}

\subsection{Synthese von Halbleiternanodrähten}

Die im Rahmen dieser Arbeit untersuchten Nanodrähte bestehen aus Halbleitermaterialien. Sie wurden in einem katalytisch getriebenen Wachstumsmechanismus, basierend auf dem VLS-Mechanismus [Wag64], hergestellt, der in dem ersten Abschnitt dieses Kapitels beschrieben wird. Hergestellt und untersucht wurden Nanodrähte aus Zinkoxid, Zinksulfid, Silizium, Mangansulfid, Cadmiumsulfid und Galliumarsenid. Die Wachstumsmechanismen der verschiedenen Materialien unterscheiden sich zum Teil von dem VLS-Mechanismus, der für das Wachstum von SiliziumNanodrähten konzipiert wurde. Auf diese Unterschiede wird in Abschnitt 2.1.2 eingegangen. Die Synthese der Nanodrähte erfolgte durch Gasphasenabscheidung. Dazu wurden mehrere horizontale Rohröfen verwendet, in denen das Ausgangsmaterial in der heißen Zone verdampft und durch ein Vektorgas zu dem kälteren Ende transportiert wird, wo es zur Abscheidung und zum anisotropen Kristallwachstum kommt. Lediglich die Galliumarsenid-Nanodrähte wurden in einer metallorganischen Gasphasenepitaxie in der Gruppe von Prof. Dr. F.J. Tegude/ Dr. W. Prost an der Universität Duisburg/Essen hergestellt.

\subsubsection{Der VLS-Mechanismus}

Bei dem VLS-Mechanismus (Vapor-Liquid-Solid) handelt es sich um ein Wachstumsmodell für katalytisch getriebenes, anisotropes Kristallwachstum. Das Modell wurde bereits 1964 von Wagner und Ellis für das Wachstum von einkristallinen Sili- 

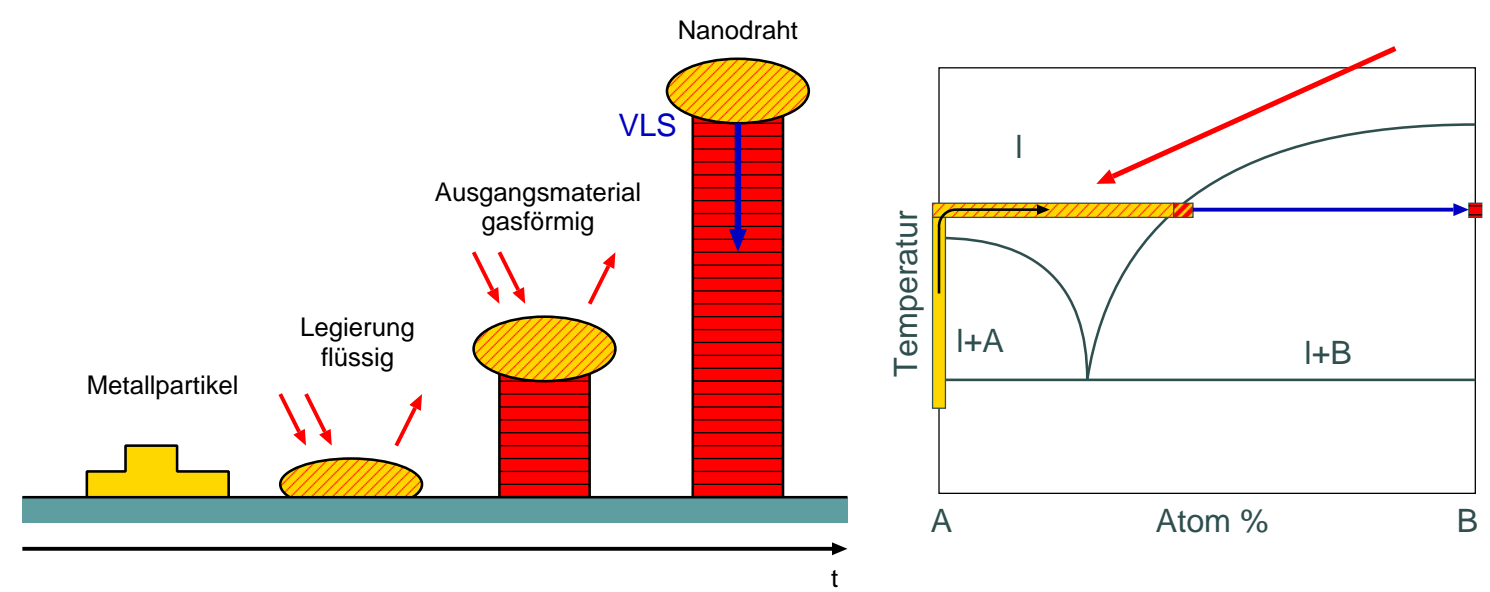

Abbildung 2.1: Wachstumsmodell für Halbleiternanodrähte. In dem VLS-Mechanismus wird das Reaktant B aus der Gasphase zugeführt, an einem Metallpartikel A präferenziell adsorbiert und geht dort in Legierung. Während weiter Ausgangsmaterial zugeführt wird unterkühlt die Legierung konstitutionell - es kommt zur Keimbildung und zum anisotropen Kristallwachstum.

ziumdrähten mit Gold als Katalysator postuliert [Wag64]. Wagner und Ellis waren damals bereits in der Lage, Drähte mit einem Durchmesser von $100 \mathrm{~nm}$ herzustellen. Auch das Wachstum nadelförmiger Zinkoxid-Kristalle wird bereits früh in der Literatur beschrieben [Sch53, Par67]. Dabei unterscheiden sich die Syntheseverfahren nicht so sehr von den heutigen.

Abbildung 2.1 zeigt schematisch das postulierte VLS-Wachstumsmodell. Ausgangspunkt des Wachstums sind Metallpartikel auf einem Substrat. Aus der Gasphase zugeführtes Ausgangsmaterial wird zunächst präferenziell an diesen adsorbiert und bildet eine Legierung. Die eutektische Temperatur der Metall-Halbleiter-Legierung ${ }^{1}$ ist dabei typischerweise niedriger als die Substrat- bzw. Wachstumstemperatur. Damit ist die Legierung mindestens bei der eutektischen Konzentration flüssig. Während fortlaufend Material aus der Gasphase zugeführt wird, unterkühlt die Legierung konstitutionell und es kommt zur Bildung eines Kristallkeims. Dies geschieht dort, wo die bei der Keimbildung aufzubringende Oberflächenenergie minimal ist: typischerweise an der Grenzfläche zum Substrat. Das stetig zugeführte Ausgangsmaterial kristallisiert an der Grenzfläche zwischen Legierungstropfen und Kristallkeim aus. Dabei ist das Metall nur katalytisch wirksam, indem es als Ort der präferenziellen

\footnotetext{
${ }^{1}$ Voraussetzung ist, dass Halbleiter und Metall ein eutektisches System bilden.
} 
Adsorption des Ausgangsmaterials agiert. Der Legierungstropfen bleibt als Kugel an dem Ende des Drahtes erhalten und wird vor der Wachstumsfront entlang getrieben. Der Kristall wächst folglich anisotrop als Draht, dessen Durchmesser durch die Größe des Legierungstropfens bestimmt wird. Der Legierungstropfen kann nach dem Wachstumsprozess an den Drähten beobachtet werden (siehe Abbildung 2.2). Über die Beschaffenheit der Metallpartikel und des Substrates lassen sich vor dem Wachstum bereits viele Eigenschaften der Nanodrähte bestimmen. Über die Größe der Metallcluster lässt sich der Drahtdurchmesser, durch die Wachstumszeit, bzw. das Angebot an Ausgangsmaterial, die Drahtlänge einstellen. Als Katalysatormetall wird zumeist Gold verwendet. Monodisperse Goldcluster unterschiedlicher Durchmesser sind kommerziell erhältlich. Werden diese als Ausgangspunkt für ein VLSWachstum verwendet, lassen sich monodisperse Nanodrähte herstellen. Mit Hilfe von Lithographieprozessen oder durch selbstorganisierte Prozesse lässt sich der Ort und die Anordnung der Nanodrähte vorausbestimmen [Fan06]. Sind zudem geeignete Epitaxiebedingungen zum Substrat erfüllt, lässt sich eine Ausrichtung der Drähte erreichen. Homoepitaktisches Wachstum von Silizium- $\mu$-Drähten wurde analog zum homoepitakischen Schichtwachstum bereits vor über 40 Jahren in der Literatur erwähnt [Wag70]. Auch heteroepitaktisches Wachstum von Halbleiternanodrähten, beispielsweise von Silizium und Germanium [Han07, Kam03], wurde bereits durchgeführt. Bei dem Wachstum von Nanodrähten ist es möglich, heteroepitaktische Übergänge zwischen Materialien mit größerer Gitterfehlpassung zu realisiern als es beim Schichtwachstum möglich ist. Der resultierende Stress an der Grenzfläche kann aufgrund der geringen lateralen Ausdehnung effektiv über eine Volumenänderung des Kristalls in den ersten Monolagen relaxieren.

Durch Kombination von Epitaxie und Strukturierung der Oberfläche mit Goldpartikeln wurde gezeigt, dass eine regelmäßige Anordnung monodisperser Nanodrähte auf großen Flächen möglich ist [Fan06, Fuh05]. Allerdings sind dazu oft niedrige Wachstumstemperaturen notwendig, da bei hohen Temperaturen die Metallcluster auf dem Substrat mobil werden können. Außerdem wurde bei hohen Wachstumstemperaturen eine Diffusion des Metalls an der Oberfläche der Drähte sowie des Substrates beobachtet, wodurch es während des Wachstums der Nanodrähte zu einer Reifung der Legierungspartikel kam [Sch04].

Auch wenn nicht alle Wachstumexperimente mit dem VLS-Modell beschrieben wer- 


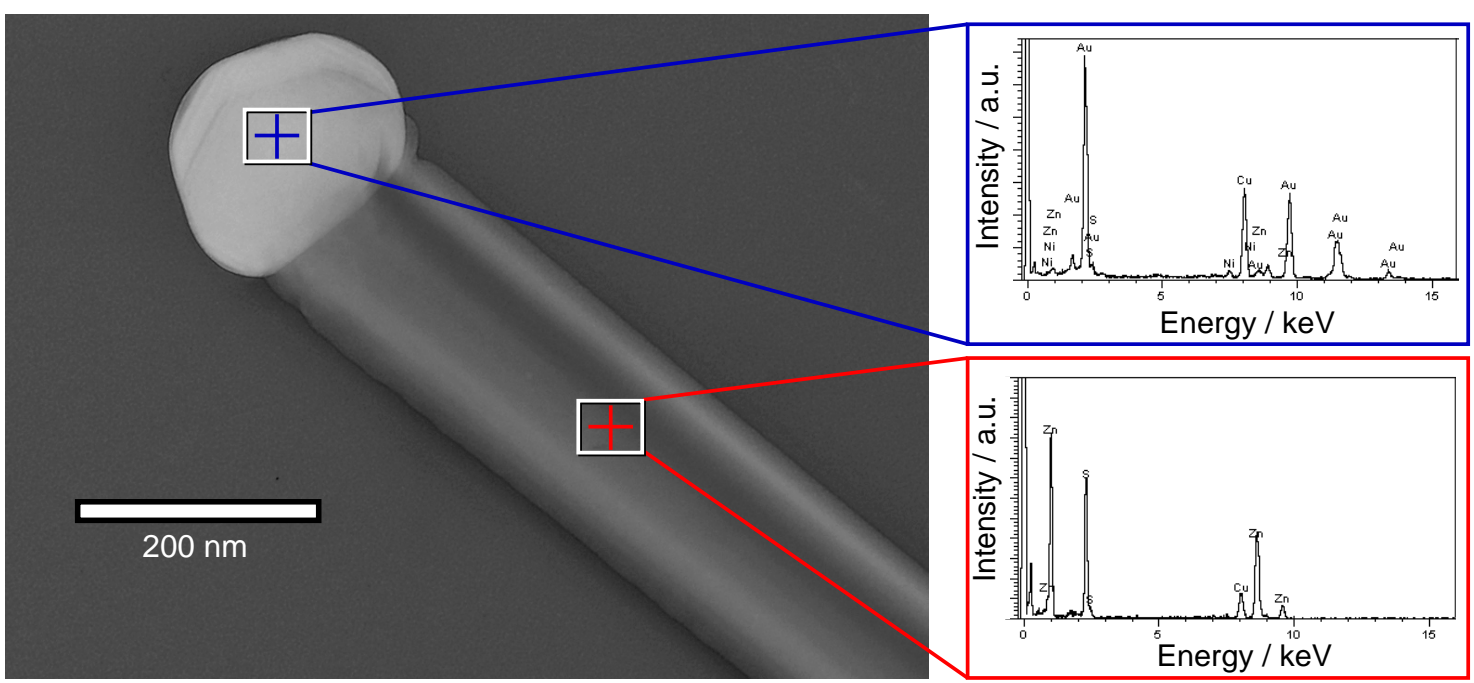

Abbildung 2.2: Transmissionselektronenmikroskopische Aufnahme eines ZinksulfidNanodrahtes. Am Ende des Drahtes ist eine facettierte Kugel zu sehen. Energiedispersive Röntgenspektroskopie zeigt, dass nur in der Kugel Gold enthalten ist, nicht aber im Draht.

den können - so wurde beispielsweise das Wachstum von Silizium-Nanodrähten mit Titan als Katalysatormetall unterhalb der eutektischen Temperatur beobachtet [Kam01] - sprechen doch viele Indizien dafür, dass das Modell zutrifft. Wie bereits erwähnt können die Legierungstropfen am Ende der Drähte beobachtet werden. Außerdem wurde der VLS-Prozess in-situ mit Hilfe eines Transmissionselektronenmikroskops (TEM) beobachtet [Ros05], wobei sich die Wachstumbedingungen deutlich von denen in üblichen Syntheseapparaturen unterscheiden.

Nanodrähte, die über einen VLS-Mechanismus hergestellt wurden, weisen oft ein großes Aspektverhältnis sowie ein hohe Kristallqualität auf. Ursache dafür ist die reinigende Wirkung des Legierungstropfens; ähnlich wie in einem Zonenschmelzprozess werden Verunreinigungen erst in den Kristall eingebaut, wenn sie in der flüssigen Phase übersättigen. Dies führt allerdings dann zu einem Problem, wenn der Halbleiter während des Wachstums kontrolliert dotiert werden soll.

Neben der Herstellung elementarer Halbleiter wurden in den vergangenen zwei Jahrzehnten, basierend auf dem VLS-Modell, Nanodrähte aus einer Vielzahl von Verbindungshalbleitern synthetisiert. Zum weiteren Verständnis des Wachstums wurde das VLS-Modell, abhängig vom Materialsystem, um einige Aspekte erweitert. 


\subsubsection{Abwandlungen des VLS-Mechanismus Sauerstoffunterstütztes Wachstum von Silizium-Nanodrähten}

Um Silizium-Nanodrähte wurde ein amorpher $\mathrm{SiO}_{2}$-Mantel beobachtet, dessen Dicke größer als eine natürliche Oxidschicht ist [Zha03]. Folglich muss dieser Mantel bereits während des Wachstums entstanden sein. Basierend auf dieser Beobachtung wurde ein Modell eingeführt, bei dem als Katalysator eine $\mathrm{Si}_{n} \mathrm{O}_{m}$-Legierung dient. Der Schmelzpunkt dieser Legierung ist niedriger als der von reinem Silizium und $\mathrm{SiO}_{2}$. An der Wachstumsfront kommt es bei der Erstarrung zu einer Phasenseparation von Silizium und Siliziumoxid. Das Silizium wächst einkristallin als Kern und wird vom Siliziumoxid umgeben. Unterstützt wird dieses Modell von der Beobachtung, dass dieses Wachstum auch ohne Metallkatalysator stattfindet [Dew07, Zha03].

\section{Wachstum von II-VI-Halbleiternanodrähten am Beispiel von Zinkoxid}

Bei der Synthese von Verbindungshalbleitern trifft die Voraussetzung eines binären, eutektischen Phasendiagramms, wie es zu der Beschreibung des VLS-Mechanismus verwendet wird, nicht zu. Die ternären Phasendiagramme, beispielsweise von AuZn-O und Au-Zn-S, sind oft nicht bekannt. In der Literartur wird zur Erklärung des Wachstums auf pseudobinäre Systeme verwiesen, wobei das Metall die eine, das Halbleitermaterial die andere Phase darstellt [Dua00].

Die Einführung eines pseudobinären Systems setzt voraus, dass Halbleitermaterial und Katalysator in der kristallinen Phase nicht mischbar sind. Für das System ZnS$\mathrm{Au}$, das zumindest aus chemischer Sicht dem System ZnO-Au ähnlich ist, kann dies zutreffen. Es gibt Hinweise aus der Geologie, wo bei der Mineralisierung von Zink und Gold eine Phasenseparation in kubisches ZnS (Sphalerit) und Gold beobachtet wurde. Gelöst in dem Sphalerit wurden metallische Verunreinigungen gefunden (Fe, $\mathrm{Cd}$, Sb und $\mathrm{Cu}$ ), allerdings kein Gold [Pal97].

Bei den Temperaturen, die im Rahmen dieser Arbeit bei der Synthese von Nanodrähten verwendet wurden ${ }^{2}$, ist Zinkoxid in der Gasphase zu einem großen Teil dissoziert [Gra76, Mak03]. Da das Ausgangsmaterial über die Gasphase zugeführt wird, ist hier eine pseudobinäre Beschreibung nicht mehr gerechtfertigt. Im Fol-

\footnotetext{
${ }^{2}$ Die Temperatur des Ausgangsmaterials beträgt hier $1350{ }^{\circ} \mathrm{C}$.
} 
genden soll, basierend auf ähnlichen Phasendiagrammen und Beobachtungen, ein ternäres Phasendiagramm für das System Au-Zn-O vorgeschlagen werden. Ausgangspunkt sind die drei binären Phasendiagramme.

Sauerstoff und Gold sind in der flüssigen Phase nur bis zu einem Verhältnis kleiner als $10^{-4}$ mischbar [Oka90]. Das komplette Phasendiagramm des Systems ist nicht bekannt. Es wird angenommen, dass ein Gleichgewichtszustand zwischen einer goldreichen, flüssigen Phase und der kristallinen Phase nahe dem Schmelzpunkt von Gold bei $1064{ }^{\circ} \mathrm{C}$ existiert [Bor06b]. Das Phasendiagramm Zn-O ist ebenfall nicht bekannt. Es werden entsprechend zwei ternäre Reaktionen angenommen: eine bei hoher Zinkkonzentration nahe dem Schmelzpunkt von reinem Zn bei $420{ }^{\circ} \mathrm{C}$ und eine weitere nahe reinem Zinkoxid. Bei mittleren Konzentrationen resultiert daraus eine Mischungslücke. Das Phasendiagramm Zn-Au ist hingegen gut bekannt [Oka90]. Neben einer Vielzahl intermetallischer Phasen gibt es zwei eutektische Punkte, von denen der bei höherer Goldkonzentration (bei $683{ }^{\circ} \mathrm{C}$ und 33,5 at.\% Zink, $\mathrm{E}_{1}$ in Abbildung 2.3 b) von besonderem Interesse für das VLS-Wachstum ist. Eine ternäre kristalline Phase aus Au-Zn-O ist nicht bekannt. Ausgehend von der Annahme, dass die intermetallischen Phasen von Gold und Zink ebensowenig mit Zinkoxid mischbar sind wie Gold und Zink selbst, wird nun ein isothermes Phasendiagramm bei etwa $550{ }^{\circ} \mathrm{C}$ vorgeschlagen, wie es in Abbildung 2.3 a) dargestellt ist.

In grau eingefärbt sind Zwei-Phasen-Bereiche mit den Nummern 1-5, wobei deren Breite dem Phasendiagramm von Au-Zn entnommen ist. Die Bereiche markieren die Phasen $\mathrm{Au}+\mathrm{ZnO}(1), \mathrm{AuZn}+\mathrm{ZnO}(2), \mathrm{AuZn}_{3}+\mathrm{ZnO}(3), \mathrm{AuZn}_{4}+\mathrm{ZnO}$ (4) und $\mathrm{L}+\mathrm{ZnO}(5)$, wobei $\mathrm{L}$ die oben beschriebene zinkreiche flüssige Legierung ist. Die Bereiche dazwischen sind Drei-Phasen-Gebiete. Da das VLS-Wachstum unter zinkreichen Bedingungen stattfindet [Wan04a], wird die obere Hälfte des ternären Phasendiagramms nicht weiter betrachtet.

Von besonderem Interesse für das VLS-Wachstum ist der Drei-Phasen-Bereich zwischen 1 und $2(\mathrm{Au}+\mathrm{AuZn}+\mathrm{ZnO})$. Ausgehend von dem binären eutektischen Punkt $\mathrm{E}_{1}$ muss eine ternäre Liquiduslinie zu einem ternären eutektischen Punkt $\mathrm{E}_{T}$ $(\mathrm{L} \leftrightharpoons \mathrm{Au}+\mathrm{AuZn}+\mathrm{ZnO})$ führen, wobei die eutektische Temperatur hier geringer sein muss als die des binären Eutektikums bei $\mathrm{E}_{1}$. Wie in Abbildung $2.3 \mathrm{~b}$ ) dargestellt ist, muss ein zweiter Pfad von einem pseudobinären Eutektikum zwischen 

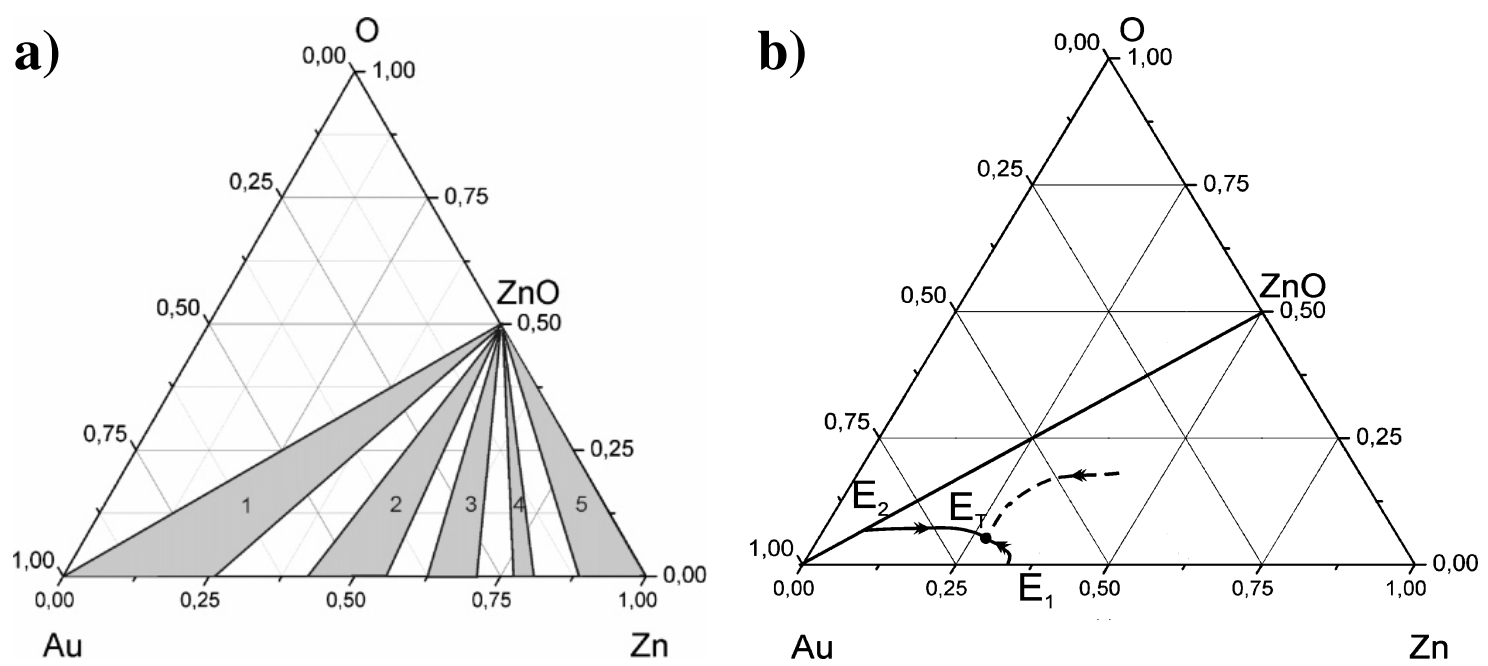

Abbildung 2.3: (a) Isothermes Phasendiagramm von Au-Zn-O bei $\approx 550{ }^{\circ} \mathrm{C}$. Zwei-PhasenBereiche sind in grau gekennzeichnet: (1) $\mathrm{Au}+\mathrm{ZnO}$, (2) $\mathrm{AuZn}+\mathrm{ZnO}$, (3) $\mathrm{AuZn}_{3}+\mathrm{ZnO}$, (4) $\mathrm{AuZn}_{4}+\mathrm{ZnO}$ und (5) L $+\mathrm{ZnO}$. Die weißen Bereiche sind Drei-Phasen-Bereiche. (b) Liquiduslinie im vorgeschlagenen ternären Phasendiagramm: von dem binären Au-AuZn Eutektikum bei $\mathrm{E}_{1}$ $\left(683{ }^{\circ} \mathrm{C}\right)$ muss die Liquiduslinie zu einem ternären Eutektikum $\mathrm{E}_{T}\left(\mathrm{Au}-\mathrm{AuZn}-\mathrm{ZnO},<683{ }^{\circ} \mathrm{C}\right)$ führen. Ebenso existiert eine Linie vom pseudobinären Eutektikum $\mathrm{E}_{2}\left(\mathrm{Au}-\mathrm{ZnO},<1069{ }^{\circ} \mathrm{C}\right) \mathrm{zu}$ $\mathrm{E}_{T}$.

$\mathrm{Au}$ und $\mathrm{ZnO}\left(\mathrm{E}_{2}\right)$ bei hoher Goldkonzentration ebenso zu diesem ternären Eutektikum führen. Ein isothermer Ausschnitt des Phasendiagramms bei $1100{ }^{\circ} \mathrm{C}$ ist in Abbildung 2.4 dargestellt. Da Zink und Zinkoxid in der flüssigen Phase nicht mischbar sind, muss eine ternäre Mischungslücke in der flüssigen Phase vorhanden sein. Mit sinkender Goldkonzentration verzweigt die goldreiche Liquidusphase L in eine zinkoxidreiche Phase L' und in eine zinkreiche Phase L". Nahe reinem Zinkoxid ist ein Zwei-Phasen-Bereich aus Zinkoxid und L', und zwischen Zinkoxid und Zink ist ein Drei-Phasen-Gebiet $(\mathrm{ZnO}+\mathrm{L}$ ' $+\mathrm{L}$ ") $)$ zu finden. Obgleich eine Entmischung der flüssigen Phase ohne Zweifel stattfindet, ist in Abbildung 2.4 die Lage des kritischen Punktes, an dem die Entmischung einsetzt, rein hypothetisch gewählt.

Treffen nun während eines VLS-Wachstums Zink und Sauerstoff auf eine Goldkugel, schmilzt die Kugel und nimmt Zink und Sauerstoff auf. Die Zusammensetzung der flüssigen Legierung startet in Abbildung 2.4 bei reinem Gold und mündet bei zunehmender Zink- und Sauerstoffkonzentration in dem Bereich L'. Schließlich übersättigt die Legierung und Zinkoxid scheidet aus. Solange Zink und Sauerstoff kontinuierlich 


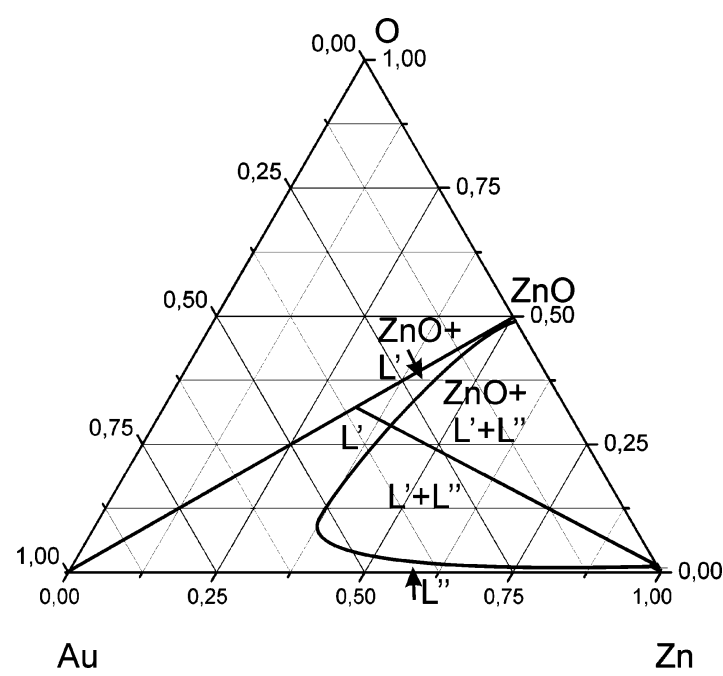

Abbildung 2.4: Isothermes Phasendiagramm von Au-Zn-O bei $1100^{\circ} \mathrm{C}$.

zugeführt werden und die Temperatur konstant bleibt, scheidet weiter Zinkoxid aus. Beim Abkühlen wird die Legierung wieder goldreich und der Legierungstropfen beginnt zu kristallisieren. Bei weiterem Angebot von Zink kann, solange die minimale Liquidustemperatur nicht unterschritten wird, ein Teil der Legierung flüssig bleiben. Sauerstoff kann von der Legierung dabei nicht mehr aufgenommen werden. So wird, wenn der Prozess im Gleichgewicht stattfindet, der ternäre eutektische Punkt erreicht. Hier entmischt die Legierung in Au, AuZn und ZnO. Aus der Literatur ist bekannt, dass bei ternären eutektischen Reaktionen oft nur zwei feste Phasen ausscheiden [Han52]. In diesem Fall ist es gut möglich, dass sich eine AuZn-Phase nicht bildet, denn in EDX-Untersuchungen konnte kaum Zink in dem Legierungstropfen nachgewiesen werden [Bor06a].

\section{Wachstum von III-V-Halbleiternanodrähten am Beispiel von GaAs}

Bei der Synthese von Heteroübergängen zwischen Indiumarsenid und Indiumphosphid [Bjö02] sowie zwischen Indiumarsenid und Galliumarsenid [Ohl02] in Nanodrähten wurden atomar scharfe Übergänge beobachtet. Wie es Persson et. al. [Per04] beschreiben, kann dies mit einem VLS-Mechanismus nicht erklärt werden. Dort würde für das erste System bei einem Austausch des zugeführten Reaktants die Konzentration des Arsens in der Legierung zunächst kontinuierlich abnehmen, 
während die Konzentration von Phosphor zunimmt. Dies würde zu einem kontinuierlichen Übergang führen - insbesondere bei diesem mischbaren System. Aus der Beobachtung, dass die Menge an Gallium, die in dem erstarrten Goldlegierungstropfen nachgewiesen wurde, gerade der Löslichkeit von Gallium in kristallinem Gold bei der Wachstumstemperatur entspricht, wird geschlossen, dass der Legierungspartikel während des Wachstums kristallin ist. Arsen konnte in dem Legierungspartikel nicht nachgewiesen werden. Basierend auf dem Vorschlag von Samuelson, dass das Gruppe-II-Element über die Oberfläche und das Gruppe-VI-Element durch den Legierungspartikel zur Wachstumsfront gelangt [Sam04], wurde ein kinetisches Wachstumsmodell aufgestellt. Mit Hilfe des Wachstumsmodells konnte unter Annahme eines kristallinen Legierungspartikels das Wachstum von GalliumarsenidNanodrähten simuliert werden [Per04].

\section{Wachstum von Nanodrähten aus der Schmelze: ein umgekehrtes VLS- Wachstum}

In einigen Untersuchungen zum Wachstum von Zinkoxid-Nanodrähten konnten keine Legierungspartikel am Ende der Drähte gefunden werden [Wan04a, Fan06]. Weiterführende Untersuchungen (u.a. TEM-Querschnittspräparationen des Wachstumssubstrates) zeigten, dass das Metall in der Grenzschicht zwischen Substrat und Draht eingelagert war. Die Ursache, dass in den beschriebenen Fällen der Legierungstropfen nicht vor der Wachstumsfront hergeschoben wird, sondern der Kristall aus der Legierung herausgeschoben wird, liegt in der benetzenden Eigenschaft des verwendeten Metalls: in einem Fall wurde Zinn verwendet, das bei den Wachstumsparametern das Substrat benetzt [Wan04a]; im anderen Fall sollte das Wachstum mit Gold als Katalysator epitaktisch auf einem Galliumnitrid-Substrat erfolgen. Bei den verwendeteten Temperaturen legierte das Metall zunächst in das Substrat ein, bevor das Wachstum der Drähte beginnt [Fan06].

\section{Wachstum an den Seitenflächen}

Zusätzlich zu dem oben beschriebenem anisotropen Wachstum kommt es in einigen Synthesemethoden zu einem Kristallwachstum an den Seitenflächen der Drähte. Die Drähte haben dann eine konische Form, deren Winkel durch das Verhältnis der Wachstumsraten gegeben ist. Über eine geeignete Modifikation des Prozesses 


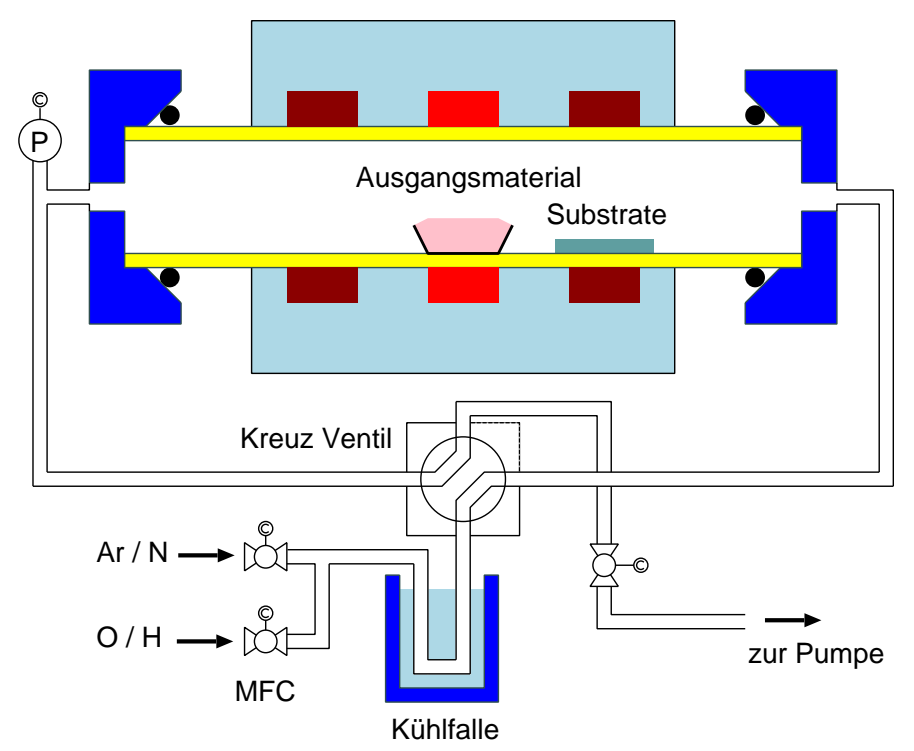

Abbildung 2.5: Apparatur zur Synthese von Halbleiternanodrähten. Die Enden eines DreiZonen-Rohrofens, die mit gekühlten Vakuumflanschen gefasst sind, können über ein Kreuzschaltventil wahlweise mit einer Vakuumpumpe oder mit einer Gasversorgung verbunden werden. Die zugeführte Gasmenge und dessen Zusammensetzung können über Massendurchflussregler eingestellt werden.

(insbesondere der Temperatur) lässt sich das Wachstum an den Seitenflächen stark unterdrücken.

\subsubsection{Nanodrahtherstellung in Göttingen}

Zur Herstellung von Halbleiternanodrähten wurden in Göttingen zwei Apparaturen aufgebaut: ein Drei-Zonen-Rohrofen, der in Abbildung 2.5 schematisch dargestellt ist, sowie ein Ein-Zonen-Rohrofen. In dem Drei-Zonen-Ofen befindet sich ein Keramikrohr (Degussit, Al23), das an den Enden mit gekühlten Vakuumflanschen gefasst ist. Die Enden des Ofens können über ein Kreuzschaltventil wahlweise mit einer Gasversorgung oder einer Vakuumpumpe verbunden werden. Zur Messung und Regulierung des Drucks stehen Vakuummesszellen und ein motorisiertes Feindosierventil zur Verfügung. Die Apparatur wurde mit einer Prozesssteuerung versehen [Sti05], in der die wesentlichen Wachstumsparameter (Temperatur, Temperaturrampen, Gasgemisch, Gasfluss, Druck und Flussrichtung) gesteuert werden können. Auf diese Weise kann eine Reproduzierbarkeit der Wachstumsbedingungen 
erreicht werden. Zur Regulierung der Gasversorgung werden zwei Massendurchflussregler verwendet, die auf Gasflüsse bis $100 \mathrm{sccm}$ und $10 \mathrm{sccm}$ kalibriert sind. Der Drei-Zonen-Ofen erlaubt Temperaturen bis $1150{ }^{\circ} \mathrm{C}$, wobei benachbarte Zonen einen Temperaturunterschied von bis zu $250^{\circ} \mathrm{C}$ haben können. Im Unterschied zu dem Drei-Zonen-Ofen ist der Ein-Zonen-Ofen nicht prozessgesteuert, dafür lassen sich Temperaturen bis $1500{ }^{\circ} \mathrm{C}$ einstellen.

Zur Synthese von Nanodrähten wird Ausgangsmaterial in der mittleren Zone des Ofens platziert, die während des Wachstums die höchste Temperatur erreicht. Die Substrate, auf denen das Wachstum der Drähte stattfinden soll, werden bei dem Drei-Zonen-Ofen in der benachbarten Temperaturzone, bei dem Ein-Zonen-Ofen am Rand des Ofens platziert. Anschließend wird das Keramik-Rohr mit Argon gespült und evakuiert. Die Enden des Keramikrohrs sind dabei so belegt, dass der Gasfluss von den Substraten zum Ausgangsmaterial gerichtet ist. Anschließend werden die gewünschten Wachstumsbedingungen eingestellt (Druck, Temperatur und Gasfluss). Sind alle Parameter erreicht wird das Kreuzschaltventil umgeschaltet, sodass ein Vektorgas das Ausgangsmaterial zu den Substraten transportieren kann. Dort befinden sich präparierte Substrate, auf denen ein VLS-Wachstum stattfindet. Das Ende der Wachstumsphase wird durch Umkehren der Flussrichtung eingeleitet. Gleichzeitig wird die Temperatur reduziert und der Druck erhöht.

Zur Präparation der Substrate wurden dünne Goldschichten $(\approx 5 \mathrm{~nm})$ auf Siliziumsubstrate thermisch aufgedampft. Die Siliziumsubstrate waren dabei von einer natürlichen Oxidschicht bedeckt. Eine Epitaxie war demnach nicht zu erwarten. Während des Aufheizens des Rohrofens zerfällt die Goldschicht typischerweise in Cluster, die als Ausgangspunkt für ein VLS-Wachstum dienen. Eine rasterelektronenmikroskopische Aufnahme eines Substrates nach der Synthese ist in Abbildung 2.6 a) zu sehen. Detaillierte Beschreibungen einzelner Wachstumsprozesse sind in [Sti05, Mü105] zu finden.

\subsubsection{Nanodrahtherstellung in Kollaborationen}

Im Rahmen dieser Arbeit wurden vergleichende Untersuchungen an Nanodrähten aus drei anderen Gruppen gemacht. Auf die Abwandlung der Wachstumsprozesse soll im Folgenden kurz eingegangen werden. 

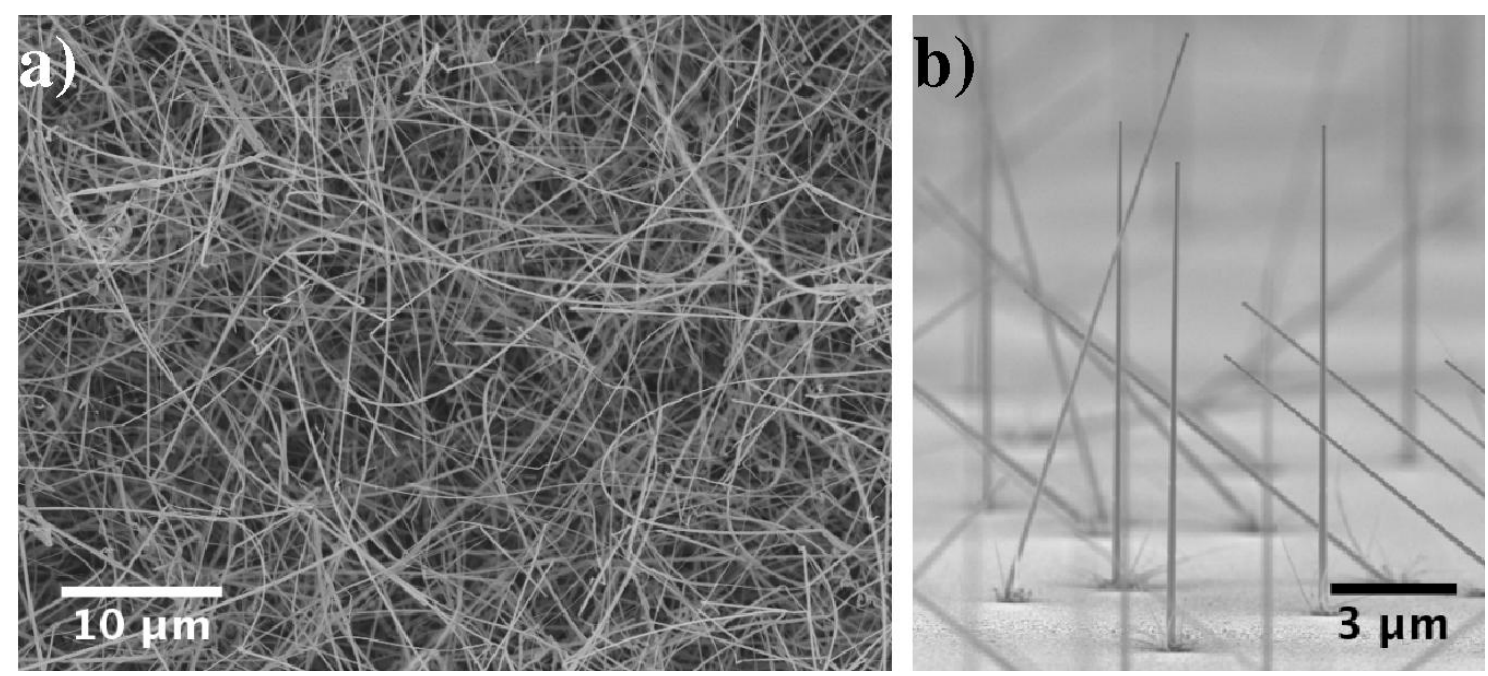

Abbildung 2.6: SEM Aufnahmen von Halbleiternanodrähten. a) Zinksulfid-Nanodrähte, wie sie in Göttingen auf einem amorphen Substrat hergestellt wurden. b) Homoepitakisch gewachsene Galliumarsenid-Nanodrähte aus der Gruppe Tegude/Duisburg.

Die in Kapitel 3.4 und 3.5 beschriebenen Experimente wurden zum Teil an Nanodrähten aus der Gruppe von Prof. Dr. Jia Grace Lu an der University of California in Irvine (UCI) bzw. an der University of Southern California (USC) gemacht. Zur Synthese von Zinkoxid-Nanodrähten wurde dort ebenfalls in einem Rohrofen durchgeführt, allerdings wurden dort zwei alternative Prozesse verwendet: (1) als Ausgangsmaterial für das Wachstum diente ein Gemisch aus Kohlenstoff und Zinkoxidpulver. Dies führt durch die Bildung eines Zinksuboxids zu einer Erhöhung des Dampfdrucks des Ausgangsmaterials und ermöglicht somit eine Synthese bei deutlich niedrigeren Temperaturen [Yao02, Cha07a]. Außerdem wurde Zinn als Katalysatormetall verwendet, was zu einem abweichenden Wachstumsprozess führt [Wan04a] (vgl. Abschnitt 2.1.2). (2) In einem alternativen Prozess, der ebenfalls in einem Rohrofen stattfand, wurde reines Zink als Ausgangsmaterial verwendet. Zinn-beschichtete Siliziumsubstrate wurden in der Öffnung einer Quarzampulle positioniert, in dessen Inneren sich das Ausgangsmaterial befand. Der notwendige Sauerstoff wurde durch Beimischung zum Trägergas aus der Gasphase zugeführt. Um zusätzlich Zink in die Gasphase zu bekommen wurde mit einem gepulsten Nd:YAGLaser die Ablation des Ausgangsmaterials unterstützt. So konnte das Wachstum von 
Zinkoxid-Nanodrähten bei einer Temperatur von nur $500{ }^{\circ} \mathrm{C}$ erreicht werden.

Analog zu dem mit (1) gekennzeichneten Prozess wurden auch Zinkoxid-Nanodrähte in der Gruppe von Prof. Dr. Quan Li an der Chinese University of Hong Kong hergestellt, die insbesondere im Zusammenhang mit der Implantation von Seltenen Erden untersucht werden (vgl. Abschnitt 4.1). Der Durchmesser dieser Nanodrähte beträgt $40 \pm 20 \mathrm{~nm}$. Näheres zu dem Herstellungsprozess ist in Ref. [Wan06] zu finden.

Die Experimente, die in Kapitel 6.1 und 4.2 beschrieben sind, wurden an Galiumarsenid-Nanodrähten durchgeführt, die in der Gruppe von Prof. Dr. F.J. Tegude/ Dr. W. Prost an der Universität Duisburg/Essen hergestellt wurden. In Duisburg wurde dazu eine kommerzielle metallorganische Dampfphasenepitaxie-Anlage (MOVPE) vom Typ AIX200RF der Firma AIXTRON verwendet. Als Ausgangsmaterial diente hier Tertiarybutylarsin (TBAs) bzw. Trimethylgallium (TMGa). Als Katalysator wurden kommerzielle monodisperse Goldnanopartikel mit einem Durchmesser von $150 \mathrm{~nm}$ (Ted Pella Inc.) verwendet. Die gewachsenen Drähte sind in Abbildung 2.6 b) abgebildet.

\subsubsection{Zusammenfassung der Wachstumsprozesse}

In den einzelnen Abschnitten wurden Modelle zum Wachstum von Halbleiternanodrähten und die Realisierung des Wachstums durch geeignete Prozesse vorgestellt. Die Modelle basieren im Grunde alle auf dem VLS-Prozess, der in den 60er Jahren für das Wachstum von Silizium-Nanodrähten postuliert wurde. Im Detail ergeben sich, beispielsweise für das Wachstum binärer Systeme, Abweichungen von dem ursprünglichen Modell. Allen Modellen gemein, und damit entscheidend für das anisotrope Kristallwachstum von Halbleiternanodrähten, ist, dass ein Ort präferenzieller Adsorption zugeführten Ausgangsmaterials vorhanden ist. Ob dieser zur Initiierung des Wachstums in Form von Metallclustern vorgegeben wird oder sich während des Wachstums selbstorganisiert ausprägt, ist unerheblich. Allerdings erlaubt die Vorgabe eines Metallclusters Kontrolle über Ort, Durchmesser und Anordnung der Nanodrähte.

Desweiteren sollte die Kinetik beim Wachstum von Nanodrähten ballistisch begrenzt sein. Bei mehrkomponentigen Materialsystemen muss dies mindestens für 
eine Komponente gelten. Folglich sind die typischen Temperaturen beim Wachstum von Nanodrähten deutlich geringer als beim Wachstum dünner Schichten, wo die Wachstumskinetik hauptsächlich diffusionsbegrenzt stattfindet. So konnte für das Wachstum von III-V-Halbleiternanodrähten gezeigt werden, dass die GruppeIII-Komponente ballistisch und durch präferenzielle Adsorption an Metallpartikeln zur Wachstumsfront gelangt und die Gruppe-V-Komponente diffusionsgetrieben über die Oberfläche dorthin gelangt. Für das Wachstum von II-VI-Halbleitern kann ähnliches gelten. Eine Betrachtung des ternären Phasendiagramms Au-Zn-O zeigt, dass die Löslichkeit von Sauerstoff in einer Au-Zn-Legierung klein ist, während zahlreiche intermetallische Au-Zn-Phasen existieren. Dies ist ein Hinweis darauf, dass auch bei diesem Wachstum die metallische Phase durch den Katalysator und die nichtmetallische Phase über die Oberfläche zur Wachstumsfront gelangt. Die Beteiligung einer weiteren Phase am Wachstum, die meist aus dem Katalysatormetall und einer der Halbleiterkomponenten besteht, führt in jedem Fall dazu, dass eine gezielte Dotierung der Halbleiternanodrähte während des Wachstums nicht möglich ist.

Die vorgestellten Wachstumsprozesse basieren darauf, dass das Ausgangsmaterial durch ein Vektorgas zum Wachstumssubstrat befördert wird. Der Unterschied besteht in den Verfahren, wie das Ausgangsmaterial in die Gasphase gebracht wird. Dies geschieht meist durch thermisches Verdampfen in einem Rohrofen. Zudem unterscheiden sich die Synthesetemperaturen. Während in Göttingen mit dem Ziel, eine hohe Kristallqualität zu erreichen, oft relativ hohe Temperaturen verwendet wurden und das Ausgangsmaterial stochiometrisch angeboten wurde, wurden in den Kollaborationen vorwiegend niedrige Temperaturen gewählt, um insbesondere monodisperse Strukturen herzustellen. Dazu war es nötig, das Ausgangsmaterial in anderen Zusammensetzungen anzubieten. In allen Prozessen wurde außerdem ein Metallkatalysator (Au bzw. Sn) auf das Wachstumssubstrat aufgebracht, wahlweise als dünne Schichten, die während des Aufheizprozesses in Cluster zerfallen, oder in Form von monodispersen Clustern.

Mit den vorgestellten Prozessen konnten Nanodrähte mit einem Durchmesser zwischen $4 \mathrm{~nm}$ und $500 \mathrm{~nm}$ und einer typischen Länge von einigen Mikrometern hergestellt werden, die, wenn Epitaxiebedingungen vorliegen, zueinander ausgerichtet sind. Dabei wurde insbesondere das Wachstum von Zinkoxid, aber auch die Mate- 
rialsysteme ZnS, GaAs, CdS, MnS und Si untersucht.

\subsection{Modifikation von Halbleiternanodrähten}

\subsubsection{Ionenimplantation}

Bei der Bestrahlung eines Festkörpers mit energetischen Ionen werden diese an den Elektronen und Kernen entlang ihrer Trajektorie gestreut. Der Wirkungsquerschnitt der beiden Streuvorgänge hängt dabei insbesondere von der Ionenenergie, der Ionenmasse sowie der Masse der Kristallatome ab. Bei hohen Ionenenergien und niedrigen Massen ist die Streuung an den Elektronen des Festkörpers, das so genannte elektronische Bremsvermögen, dominant. Bei niedrigen Ionenenergien und größeren Massen steigt der Wirkungsquerschnitt der Kerne, das nukleare Bremsvermögen, an. Ist der Energieübertrag an das Kristallsystem hinreichend groß, wird eine Streuung zur Erzeugung von Kristalldefekten führen. Üblicherweise wird eine Stoßkaskade erzeugt, bevor das Ion seine entgültige Position erreicht. Die Reichweite der eingebrachten Ionen wird durch ein Wechselspiel der beiden Bremsvorgänge bestimmt [May70]. In der Stoßkaskade kann auf die Gitteratome soviel Energie übertragen werden, dass sie für einzelne Atome in der Nähe der Oberfläche ausreichend ist, um den Festkörper verlassen zu können. Dies wird auch als Ionenzerstäubung oder Sputtereffekt bezeichnet. Mit dem Montecarlo basierten Softwarepaket TRIM lässt sich die Stoßkinetik und damit die Reichweite von Ionen, die Verteilung von Defekten und auch der Sputtereffekt in verschiedenen Materialien simulieren [Zie08]. Dabei werden Kollisionskaskaden für einzelne Ionen berechnet; der Einfluss der Kristallstruktur auf die Eindringtiefe der Ionen (Kanalisierung) wird ebensowenig berücksichtigt wie das dynamische Ausheilen der Defekte.

\section{Dotierung durch lonenimplatation}

Um die elektrischen und optischen Eigenschaften der Halbleiternanodrähte zu verändern, werden in diese durch Ionenimplantation gezielt Fremdatome eingebracht. Dazu stehen in Göttingen mehrere Beschleuniger zur Verfügung, die den Energiebereich zwischen $100 \mathrm{eV}$ und $500 \mathrm{keV}$ abdecken [Uhr05].

Der wesentliche Vorteil der Dotierung durch Ionenimplantation gegenüber anderen 
Verfahren wie der Dotierung während des Wachstums oder der Eindiffusion, ist die präzise Kontrolle über die eingebrachte Dosis. Durch eine geeignete Wahl der Ionenenergie und der Ionenfluenz lässt sich ein berechenbares Konzentrationsprofil in einem Festkörper erzeugen. Zusätzlich besteht die Möglichkeit einer Dotierung jenseits der Löslichkeitsbeschränkung. Mit der Verwendung von Masken ist zudem eine lateral strukturierte Dotierung möglich.

Allerdings geht mit dem Eindringen der Ionen in den Festkörper eine lokale Erzeugung von Kristalldefekten einher. Diese Defekte dominieren die Eigenschaften des Festkörpers und verhindern die Aktivierung des eingebrachten Dotanden, sodass ein anschließender Ausheilprozess notwendig ist.

\section{Ausheilen der Defekte}

Um die Defekte nach der Implantation auszuheilen und die Dotieratome auf Gitterplätzen zu aktivieren, wurden die Proben einer thermischen Nachbehandlung unterzogen. Abhängig vom Material wurden unterschiedliche Ausheilverfahren entwickelt. So wurden die Zinksulfid-Nanodrähte ausschließlich im Vakuum, ZinkoxidNanodrähte wahlweise auch unter Luft oder unter Sauerstoff und Galliumarseniddrähte unter Arsengegendruck getempert. Die verwendeteten Temperaturen liegen typischerweise zwischen $300{ }^{\circ} \mathrm{C}$ und $800{ }^{\circ} \mathrm{C}$, die typischen Zeiten bei $15 \mathrm{~min}$ bis $30 \mathrm{~min}$.

\subsubsection{Dispersion von Nanodrähten}

Für einige Experimente ist es notwendig, Nanodrähte vereinzelt auf einem Substrat zu untersuchen. Beispiele hierfür sind elektrische Untersuchungen, bei denen der Nanodraht auf dem Substrat liegen muss, um lithographisch Kontakte anzubringen. Auch für die Ionenimplantation ist es oft sinnvoll, die Drähte auf ein anderes Substrat zu übertragen, um Abschattungseffekte zu vermeiden.

Zur Übertragung der Drähte wurden zwei unterschiedliche Verfahren verwendet. Zum einen wurden die Nanodrähte in Isopropanol von den Wachstumssubstraten abgelöst. Die Suspension wurde dann auf gereinigte Substrate aufgeschleudert. Diese Methode hat den Vorteil, dass aus einer Mutterprobe gleiche Tochterproben erstellt werden können. Durch Wiederholung des Schleudervorgangs kann die Dichte der 
Drähte auf den Tochterproben eingestellt werden. Bei der zweiten Methode wird ein Abdruck der Mutterprobe genommen, indem das Tochtersubstrat auf dessen Oberfläche gedrückt wird. Dabei bleiben einige Drähte auf dem Tochtersubstrat zurück, ohne dass die Oberfläche der Nanodrähte durch ein Lösungsmittel potentiell kontaminiert wird.

\subsubsection{Elektrische Adressierung: Elektronenstrahllithographie}

Zur elektrischen Kontaktierung von Nanodrähten wurden mit Hilfe der Elektronenstrahllithographie Masken erstellt, durch die elektrische Leiterbahnen aufgedampft wurden. Dies ist ein vielschrittiger Prozess, der im Folgenden beschrieben wird.

Zunächst wurden die Nanodrähte wie oben beschrieben auf einem isolierenden Substrat dispergiert. Die Position der Drähte wurde relativ zu Justiermarkierungen auf dem Substrat ermittelt. Anschließend wurde ein Elektronenstrahlresist mit einer Dicke von $\approx 200 \mathrm{~nm}$ auf das Substrat aufgeschleudert. Dort, wo später die Leiterbahnen entstehen sollten, wurde der Resist mit einem Elektronenstrahl belichtet. Im folgenden Entwicklungsprozess wurden die belichteten Bereiche entfernt. Nun konnte auf die strukturierte Oberfläche ein Metall aufgedampft werden und anschließend der nicht belichtete Resist in Aceton abgelöst werden. Essentiell dabei ist, dass die belichteten Stellen einen hinreichend großen Unterschnitt haben, damit das Ätzmittel im letzten Schritt den nicht belichteten Resist angreifen kann und die Goldschicht an den Rändern der Leiterbahn begrenzt ist. Auf dem Substrat bleiben so lediglich die gewünschten Leiterbahnen zurück.

\subsection{Methoden zur Charakterisierung von Halbleiter- nanodrähten}

\subsubsection{Elektronenmikroskopische Verfahren Rasterelektronenmikroskopie (SEM)}

Rasterelektronenmikroskope haben typischerweise eine laterale Auflösung, die kleiner als $10 \mathrm{~nm}$ ist [Rei98]. Sie eignen sich dementsprechend ideal, um wesentliche Eigenschaften der Nanodrähte, wie Durchmesser, Aspektverhältnis, Ausrichtung, Morphologie, Oberflächenbedeckung und Größenverteilung, zu untersuchen. 
Zur Analyse der hergestellten Nanodrähte diente ein SEM vom Typ Leo Supra35 ${ }^{\mathrm{TM}}$ der Firma Carl Zeiss SMT AG. Das Mikroskop ist mit einer Gemini $(R)$ Säule ausgerüstet, in der neben dem Linsensystem auch ein ringförmiger Detektor zum Nachweis von Sekundärelektronen untergebracht ist. Zusätzlich verfügt es über einen klassischen Everhart-Thornley Sekundärelektronendetektor [Eve60]. Die Elektronen werden aus einer Schottky-Feldemissionskathode aus $\mathrm{ZrO} / \mathrm{W}$ extrahiert. Dieses System erlaubt Vergrößerungen zwischen 12fach und 900000fach und eine Auflösung von 2,5 nm bei $1 \mathrm{kV}$ Beschleunigungsspannung (Herstellerangabe). Ausführliche Informationen über den Aufbau des SEM sind beim Hersteller, eine Beschreibung der Detektorsysteme und der Bildentstehung in Ref. [Rei98] zu finden.

\section{Transmissionselektronenmikroskopie (TEM, HRTEM)}

Die Transmissionselektronenmikroskopie (TEM) bzw. die hochauflösende Transmissionselektronenmikroskopie (HRTEM) sind Untersuchungsmethoden, um Nanodrähte auf atomarer Skala zu untersuchen. Neben den geometrischen Eigenschaften lassen sich im TEM insbesondere Eigenschaften des Kristalls, wie dessen Kristallstruktur, die Kristallorientierung der Nanodrahtachse oder evtl. vorhandene Kristalldefekte untersuchen.

Zur Präparation der TEM-Proben wurde ein Kupfernetz, das mit einem amorphen Kohlestofffilm belegt ist, über die hergestellten Proben gestreift. Dabei blieben hinreichend viele Nanodrähte an dem Kohlestofffilm zurück.

Die Untersuchungen wurden zum Teil an einem Philips CM200-FEG-UT mit 200 kV Beschleunigungsspannung am IV. Physikalischen Institut der Universität Göttingen durchgeführt. Das Miroskop hat eine Auflösung von 0,11 nm; so ist eine atomare Auflösung möglich. Außerdem wurde ein Tecnai 20 FEG-TEM an der Chinese University in Hong Kong zur Analyse genutzt. Dieses Mikroskop verfügt zusätzlich über einen Energieanalysator der transmittierten Elektronen, sodass Energieverlustspektroskopie (EELS) betrieben werden kann. Eine Zusammenfassung von TEMAnalysetechniken an Nanostrukturen ist in Ref. [Wan03, Wan00] zu finden. 


\section{Energiedispersive Röntgenspektroskopie (EDX)}

Sowohl am TEM als auch am SEM ist energiedispersive Röntgenspektroskopie (EDX) möglich. Diese Technik ist im Prinzip eine ortsaufgelöste Röntgenfluoreszenzanalyse [Rei98]. Dabei wird der Elektronenstrahl des Mikroskops dazu genutzt, die Probe zur Emission von charakteristischen Röntgenstrahlen anzuregen. Diese werden von einem seitlich angebrachten Detektor erfasst, der die Energie und Intensität der eintreffenden Röntgenphotonen misst. Die energiedispersive Röntgenspektroskopie ermöglicht eine ortsaufgelöste, qualitative und quantitative Elementanalyse. Die laterale Auflösung einer solchen Messung wird allerdings durch die Eindringtiefe des Elektronenstrahls in das Material begrenzt. Abhängig von der Energie der Elektronen und der Morphologie der Probe wird der Durchmesser der Anregungsbirne, aus der die Röntgenstrahlen stammen, bis zu einem Mikrometer groß. Ist die angeregte Struktur dünn, wie es im TEM der Fall ist, ist die Ortsauflösung wesentlich besser $(<5 \mathrm{~nm})$. Aufgrund der erzeugten Bremsstrahlung ist die quantitative Nachweiswahrscheinlichkeit größer als $1 \%$. Leichte Elemente können nicht nachgewiesen werden.

\subsubsection{Optische Spektroskopie}

Durch extrinsische und intrinsische Defekte können Zustände in der Bandlücke eines Halbleiters erzeugt werden. Eine Standardmethode zur Charakterisierung dieser Zustände ist die Photolumineszenzspektroskopie (PL) bzw. die Kathodolumineszenzspektroskopie (CL). Diese Untersuchungsmethoden sind hinsichtlich der Art und Konzentration von Defekten, die strahlende Rekombinationszentren in der Bandlücke bilden, sehr sensitiv. Die energetische Lage dieser Zustände relativ zu den Bandkanten und die zugehörigen Aktivierungsenergien sind ebenfalls durch Lumineszenzmessungen zugänglich. Darüber hinaus bieten zeitaufgelöste Messungen einen Einblick in die Relaxaktionsprozesse [Stö00]. Indirekt lassen sich anhand der Intensitäten Aussagen über die Kristallinität ableiten. Ein defektreicher Kristall bietet eine hohe Konzentration nichtstrahlender Rekombinationszentren (Killerzentren), was zu einer Verringerung der Intensität führt. Schließlich ist die Lumineszenzspektroskopie eine berührungslose und zerstörungsfreie Untersuchungsmethode. Eine detaillierte Darstellung der Lumineszenzspektroskopie ist in Ref. [Vij98] 


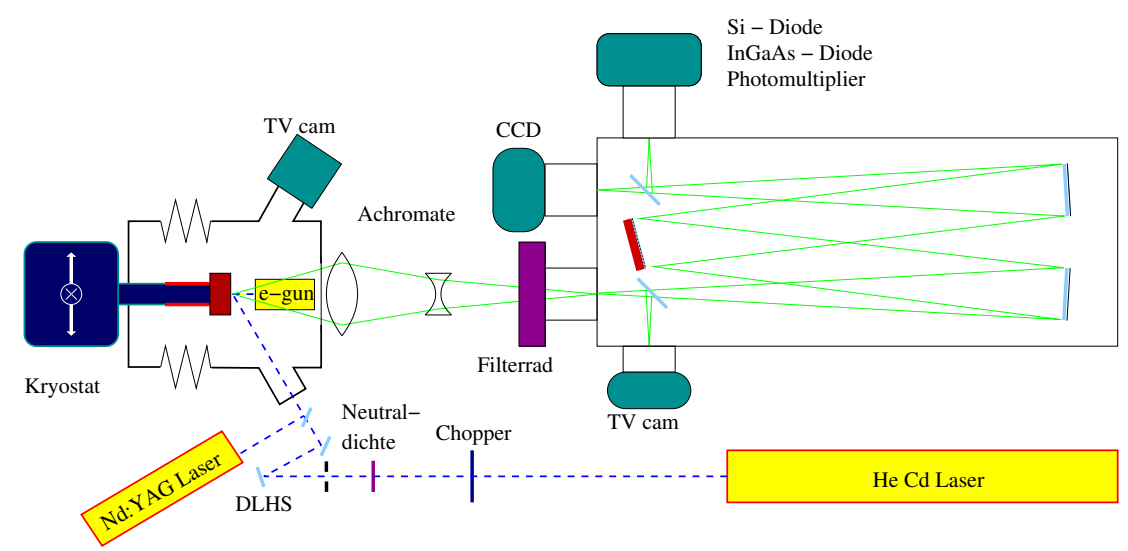

Abbildung 2.7: Schema der Lumineszenzapparatur: Anregung der Lumineszenz erfolgt über eine Elektronenkanone (CL) oder einen Laserstrahl (PL), der über dielektrische Hochleistungsspiegel (DLHS) auf die Probe gelenkt wird. Die Intensität des Lasers lässt sich über Neutraldichtefilter (ND) einstellen. Der Probenhalter ist kühlbar bis $15 \mathrm{~K}$ auf einem Manipulator in einer Vakuumkammer. Die Lumineszenz wird über ein Paar Achromate langpassgefiltert in den Spektrographen eingekoppelt. Dort wird das Licht an einem Gitter gebeugt und spektral zerlegt auf einen der beiden Austrittsspalte abgebildet. Dort stehen auswechselbare Detektoren für unterschiedliche Wellenlängenbereiche zur Verfügung.

zu finden.

\section{Die Lumineszenzapparatur}

Abbildung 2.7 zeigt eine schematische Darstellung der Lumineszenzapparatur, mit der die Untersuchungen in der vorliegenden Arbeit durchgeführt wurden. Die Apparatur ermöglicht sowohl CL- als auch PL-Untersuchungen in dem Wellenlängenbereich zwischen $190 \mathrm{~nm}$ und $1500 \mathrm{~nm}$. Die Proben befinden sich in einer Vakuumkammer auf einem Mehrfachprobenhalter, dessen Position über einen Manipulator verstellt werden kann. Der Probenhalter ist an einer zweistufigen HeliumKühlmaschine der Firma CTI Cryogenics (Modell 22C/350C) angebracht. Der Probenhalter und die zweite Kühlstufe sind von einem Aluminium-Kühlschild umgeben. Durch eine Heizwendel an der zweiten Kühlstufe lassen sich Probentemperaturen zwischen $15 \mathrm{~K}$ und $300 \mathrm{~K}$ einstellen. Zur Regulierung der Temperatur wird ein Controller der Firma LakeShore (Modell 331) verwendet, zur Temperaturmessung dienen zwei Siliziumdioden (Typ DT 430). Zur Anregung der PL wird ein HeliumCadmium-Dauerstrichlaser (Firma TOPAG Typ HCL-30Ymc, 25 mW, 325 nm) und 
ein gütegeschalteter, frequenzvervierfachter Nd:YAG-Laser (Ultra CFR der Firma Quantel/Big Sky Laser Technologies Inc., 266 nm, 5 ns, 3 MW) verwendet. Die Laserstrahlen werden über dielektrische Hochleistungsspiegel (DLHS) in die Vakuumkammer eingekoppelt, wobei die Anregungsintensität über einem Neutraldichtefilter (ND) variiert werden kann. Bei der CL wird eine Elektronenkanone vom Typ EQ22 der Firma SPECS zur Anregung verwendet (100 eV - $5 \mathrm{keV}, 0.01 \mu \mathrm{A}-250 \mu \mathrm{A})$. Über ein Paar UV-beschichteter Achromate wird die Lumineszenz auf den Eintrittsspalt des Monochromators abgebildet. Bei dem Monochromator handelt es sich um einen Czerney-Turner-Monochromator vom Typ 1000M der Firma Jobin-Yvon Horiba mit einer fokalen Länge von einem Meter und einer Auflösung von 8 pm (Herstellerangabe). Dieser ist mit einem motorisierten Filterwechsler ausgestattet, sodass während einer Messung Langpassfilter in den Strahlengang eingefahren werden können, um höhere Beugungsordnungen auszublenden. Der Eintrittsspalt lässt sich zur Justage der Linsen mit einem Periskop aus dem Inneren des Monochromators abbilden. Es gibt zwei Austrittsspalte, zwischen denen mit einem elektronisch einschwenkbarem Spiegel gewechselt werden kann. Für die Dispersion des einfallenden Lichtes gibt es drei Gitter mit unterschiedlichem Linienabstand und Blaze (1200 l/mm und $250 \mathrm{~nm}, 600 \mathrm{l} / \mathrm{mm}$ und $1 \mu \mathrm{m}, 100 \mathrm{l} / \mathrm{mm}$ und $450 \mathrm{~nm}$ bei einer Gittergröße von 110 mm x $110 \mathrm{~mm}$ ).

Als Detektoren stehen eine stickstoffgekühlte CCD-Kamera, ein Photomultiplier sowie eine Silizium- und eine Indiumgalliumarsenid-Diode zur Verfügung. Die CCDKamera (Typ Spektrum One der Firma Jobin-Yvon Horiba, 1024x128 Pixel) befindet sich ständig an dem axialen Austrittsspalt. Ihre Quanteneffizienz ist in dem Wellenlängenbereich von $220 \mathrm{~nm}$ bis $940 \mathrm{~nm}$ größer als $50 \%$. Abhängig von der Wahl des Gitters lässt sich mit der CCD-Kamera ein Wellenlängenbereich zwischen $20 \mathrm{~nm}$ beim 1200er Gitter und $250 \mathrm{~nm}$ beim 100er Gitter in einem Bild detektieren. Die übrigen Detektoren können an dem seitlichen Austrittsspalt angebracht werden. Der Photomultiplier (PMT) (TypR928 der Firma Hamamatsu) wird im Photoncounting-Modus betrieben. Dazu werden die Signale des PMT linear verstärkt und in einem Constant Fraction Discriminator in TTL Pulse umgewandelt, die an einen Digitaleingang (Spectraq 2 der Firma Jobin-Yvon Horiba) weitergeleitet werden. Der Photomultiplier hat eine hohe Quanteneffizienz zwischen $190 \mathrm{~nm}$ und $750 \mathrm{~nm}$. Die Siliziumdiode eignet sich aufgrund ihrer Quanteneffizienz 
für den Wellenlängenbereich zwischen $500 \mathrm{~nm}$ und $1200 \mathrm{~nm}$, die InGaAs-Diode für den Bereich von $800 \mathrm{~nm}$ bis $1700 \mathrm{~nm}$. Die Signale der Dioden und des Photomultipliers lassen sich einerseits bei moduliertem Anregungssignal zur Verbesserung des Signal-Rauschverhältnisses Lockin verstärken (Lockinverstärker der Firma Stanford Research System Typ SR830) oder bei nicht moduliertem Signal über einen A/D Wandler direkt einlesen.

Die Kalibrierung der Apparatur wurde bei niedriger Aufösung mit der nullten Beugungsordnung des Gitters und geschlossenem Spalt vorgenommen. Bei hoher Auflösung wurde eine Quecksilberdampflampe der Firma LOT-Oriel Instruments (Oriel Model 6035) verwendet. Hierbei handelt es sich um eine Stiftlampe, die unter vermindertem Argondruck mit Quecksilber im natürlichen Isotopenverhältnis gefüllt ist. Die Wellenlänge der Emissionslinien dieser Lampe wurde [San96] entnommen.

Neben dieser Apparatur wurden für die Untersuchungen Lumineszenzapparaturen der Gruppe Dr. T. Voss/ Prof. Dr. J. Gutowski an der Universität Bremen und der Gruppe Prof. Dr. W. Heimbrodt/ Prof. Dr. J.P. Klar an der Universität Marburg genutzt. In Bremen wurden insbesondere Untersuchungen an einzelnen Nanodrähten sowie Kurzzeitdynamik der Lumineszenz in einem Mikrophotolumineszenzaufbau durchgeführt. Zur Anregung dient hier ein frequenzverdoppelter Titan-Saphir-Laser (350 nm, 100 fs, $82 \mathrm{MHz}$ ). Der Bereich der optischen Anregung hat einen Durchmesser von $\approx 2 \mu \mathrm{m}$. Die zeitaufgelösten Messungen wurden mit der Methode des zeitkorellierten Einzelphotonennachweises durchgeführt. An der Apparatur in Marburg wurde insbesondere die Lumineszenzdynamik von langlebigen optischen Übergängen untersucht. Hierzu wurde die Lumineszenz mit der dritten harmonischen eines gütegeschalteten, gepulsten Nd:YAG-Lasers (355 nm, 3,5 ns, $10 \mathrm{~Hz}$ ) angeregt und die Relaxation mit einer lichtverstärkten CCD-Kamera (Andor, iCCD) beobachtet. Dies erlaubt Zeitauflösungen von $\approx 1,5$ ns.

\subsubsection{Elektrische Leitfähigkeit}

Zur Messung der elektrischen Leitfähigkeit wurden an den Enden der Nanodrähte, wie in Abschnitt 2.2.3 beschrieben, elektrische Leiterbahnen angebracht die zu makroskopischen Kontaktflächen führen. Diese makroskopischen Kontaktflächen wur- 
den entweder direkt in einem Spitzenmessplatz adressiert oder durch das Anbringen von Bonddrähten zu größeren Kontaktflächen weitergeführt.

Von den Nanodrähten wurden Strom-Spannungskennlinien aufgenommen. Dazu wurden unterschiedliche Geräte verwendet (Keithley 2400 source/meter, HP 4515B Semiconductor Parameter Analyzer). Die typischen, noch messbaren Ströme liegen im Bereich $\approx 100$ fA.

Neben der Leitfähigkeit der Nanodrähte wurde Untersuchungen zur Ladungsträgermobilität und -konzentration sowie zur Art der Ladungsträger durchgeführt. Dazu wurden die Nanodrähte zu Feldeffekttransistoren prozessiert. Dabei dienten Kontakte an den Enden der Nanodrähte als Emitter und Kollektor - als Basis diente entweder das Substrat oder ein zusätzlich aufgebrachter Kontakt. Eine detailierte Beschreibung zur Extraktion der Messgrößen ist in Ref. [Sze81] zu finden. 


\section{Kapitel 3}

\section{Optische Untersuchungen an Zinkoxid-Nanodrähten}

Bei dem Material Zinkoxid handelt es sich um einen II-VI-Verbindungshalbleiter. Das Material kristallisiert in der wurtzitischen Phase und hat eine direkte Bandlücke von $\mathrm{E}_{G}=3,4376 \mathrm{eV}$ bei 4,2 $\mathrm{K}$ [Mey04]. Die Bandstruktur am $\Gamma$ Punkt ist in Abbildung 3.1 gezeigt. Das Leitungsband entsteht überwiegend aus den unbesetzten 4s-Zuständen der $\mathrm{Zn}^{2+}$ Ionen und hat eine $\Gamma_{7}$-Symmetrie. Das Valenzband entsteht überwiegend aus den besetzten $2 \mathrm{p}$-Orbitalen der $\mathrm{O}^{2-}$ Ionen und ist sechsfach entartet. Durch Kristrallfeld- und Spin-Bahn-Aufspaltung wird die Entartung aufgehoben, sodass schließlich drei zweifach entartete Valenzbänder $(A, B, C)$ mit $\Gamma_{7^{-}}$, $\Gamma_{9}$ - und $\Gamma_{7}$-Symmetrie entstehen [Mey04].

Neben der großen Bandlücke werden die optischen Eigenschaften von Zinkoxid durch die hohe Exzitonbindungsenergie von $60 \mathrm{meV}$ geprägt, was effiziente exzitonische Lichtemission bei Raumtemperatur ermöglicht [Tho60]. Die Exzitonbindungsenergie in $\mathrm{ZnMgO} / \mathrm{ZnO} / \mathrm{ZnMgO}$ Quantumwells ist sogar noch größer [Sun01]. Weiterhin lassen sich Heteroübergänge erzeugen [Oht98] und durch Legierung mit Cadmium bzw. Magnesium kann die Bandlücke zwischen 2,9 eV und 4 eV stark variiert werden [Mak01]. Grundlegende optische Untersuchungen an Volumenmaterial und an dünnen Schichten erfolgten in den 1960er Jahren [Tho60]. Trotz seiner hervorragenden Eigenschaften konnte sich Zinkoxid in der Optoelektronik nicht etablieren. Dies hat insbesondere zwei Gründe. Einerseits konnte in den 1960er Jahren kein Material in der notwendigen Qualität hergestellt werden, andererseits - und dies gilt bis heute - fehlt es an einer Methode zur effektiven p-Dotierung. Diese beiden 


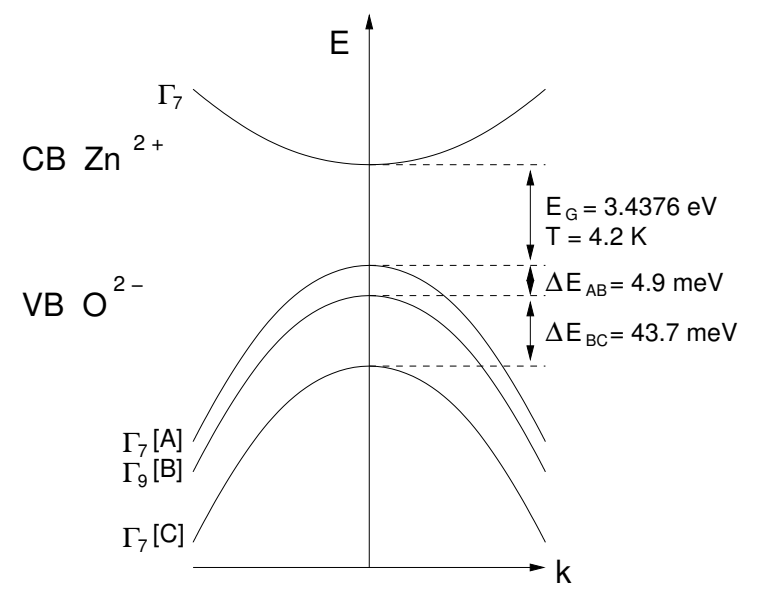

Abbildung 3.1: Bandstruktur von Zinkoxid am $\Gamma$ Punkt nach [Mey04].

Probleme sind seit etwa einer Dekade erneut Gegenstand intensiver Forschung. Eine Zusammenfassung der Eigenschaften von Zinkoxid sind unter anderem in folgenden Referenzen zu finden: [Mey04, Özg05, Kli07].

\subsection{Lumineszenz von Zinkoxid-Nanodrahtensembles bei tiefen Temperaturen}

In diesem Abschnitt sollen die grundlegenden Eigenschaften der Lumineszenz von Zinkoxid-Nanodrahtensemblen bei tiefen Temperaturen diskutiert werden. Untersucht wurden Zinkoxid-Nanodrahtproben, die in einem VLS-Prozess in Göttingen hergestellt wurden (vgl. Abschnitt 2.1.3). Abbildung 3.2 a) zeigt ein SEM-Bild eines Nanodrahtensembles direkt nach dem Wachstum. Es ist zu erkennen, dass das Wachstumssubstrat in mehreren Lagen mit Nanodrähten bedeckt ist. Die Nanodrähte haben einen Durchmesser von $\approx 150 \mathrm{~nm}$ und eine Länge von $\approx 20$ bis $50 \mu \mathrm{m}$. Unter den Nanodrähten sind im SEM-Bild mikrokristalline Strukturen zu erkennen. Der untere Teil des SEM-Bildes zeigt einen Bereich, der vor dem Wachstum nicht mit Gold bedeckt war. Hier wird kein Wachstum von Zinkoxid-Strukturen beobachtet.

Die Lumineszenzuntersuchungen wurden mit dem Aufbau durchgeführt, der in Abschnitt 2.3.2 beschrieben ist. Der Durchmesser des Laserspots auf der Probe beträgt 

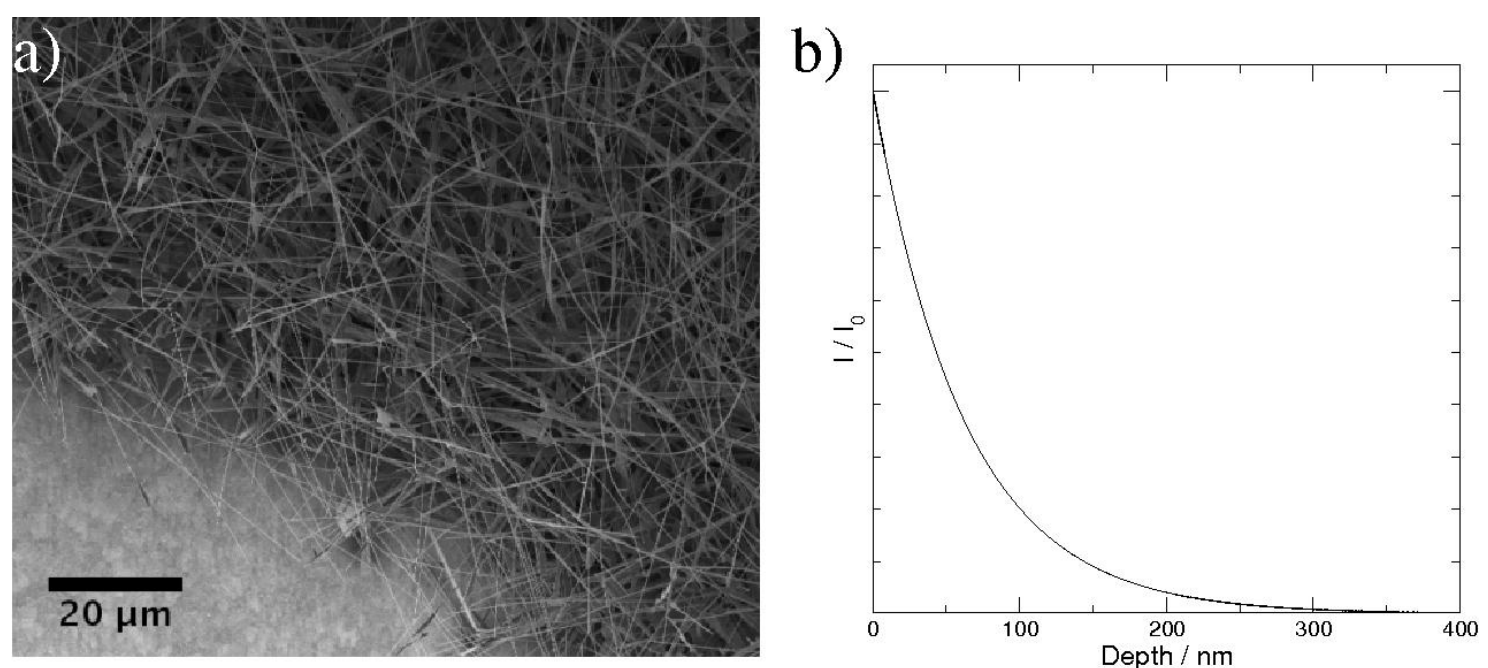

Abbildung 3.2: a) SEM-Bild eines Zinkoxid-Nanodrahtensembles nach dem Wachstum. In dem unterem Bereich befand sich vor dem Wachstum kein Gold. b) Anregungstiefe der Photolumineszenz in Zinkoxid bei $325 \mathrm{~nm}$ und $77 \mathrm{~K}$. Das Diagramm zeigt, wie die Intensität des Laserlichts entsprechend dem Lambert-Beerschen Gesetz mit der Eindringtiefe in einen Zinkoxid-Kristall exponentiell abnimmt.

etwa $3 \mathrm{~mm}$. Die Tiefe der optischen Anregung der Lumineszenz lässt sich durch die Absorption des Laserlichts annähern, die durch das Labert-Beersche Gesetz beschrieben wird (vgl. Gleichung 3.1).

$$
I(d)=I_{0} \cdot \exp (-\alpha d), \quad A=-\ln \frac{I(d)}{I_{0}}
$$

Darin bezeichnet $I(d)$ die Intensität in einer Tiefe d des Mediums, $\alpha$ den wellenlängenabhängigen Absorptionskoeffizienten und A die Extinktion. Der Absorptionskoeffizient von Zinkoxid bei $325 \mathrm{~nm}$ (Laserwellenlänge) und $77 \mathrm{~K}$ beträgt etwa $1,6 \cdot 10^{-5} \mathrm{~cm}^{-1}$ [Mut99]. Folglich ist $99 \%$ des eingestrahlten Lichts in den ersten $288 \mathrm{~nm}$ absorbiert. Abbildung 3.2 b) zeigt die Intensität des Laserlichts als Funktion der Eindringtiefe mit den angegebenen Parametern für Zinkoxid. Das angeregte Volumen (99\%) entspricht folglich dem Volumen von $\approx 10^{7}$ Nanodrähten.

In Abbildung 3.3 ist ein typisches Lumineszenz-Übersichtsspektrum eines ZinkoxidNanodrahtensembles gezeigt. Das Spektrum wurde bei einer Probentemperatur von $14 \mathrm{~K}$ mit moderater Anregungsdichte bei 3,815 eV aufgenommen. Das Spektrum zeigt, dass die gesamte Lumineszenz der Probe durch die bandkantennahen 


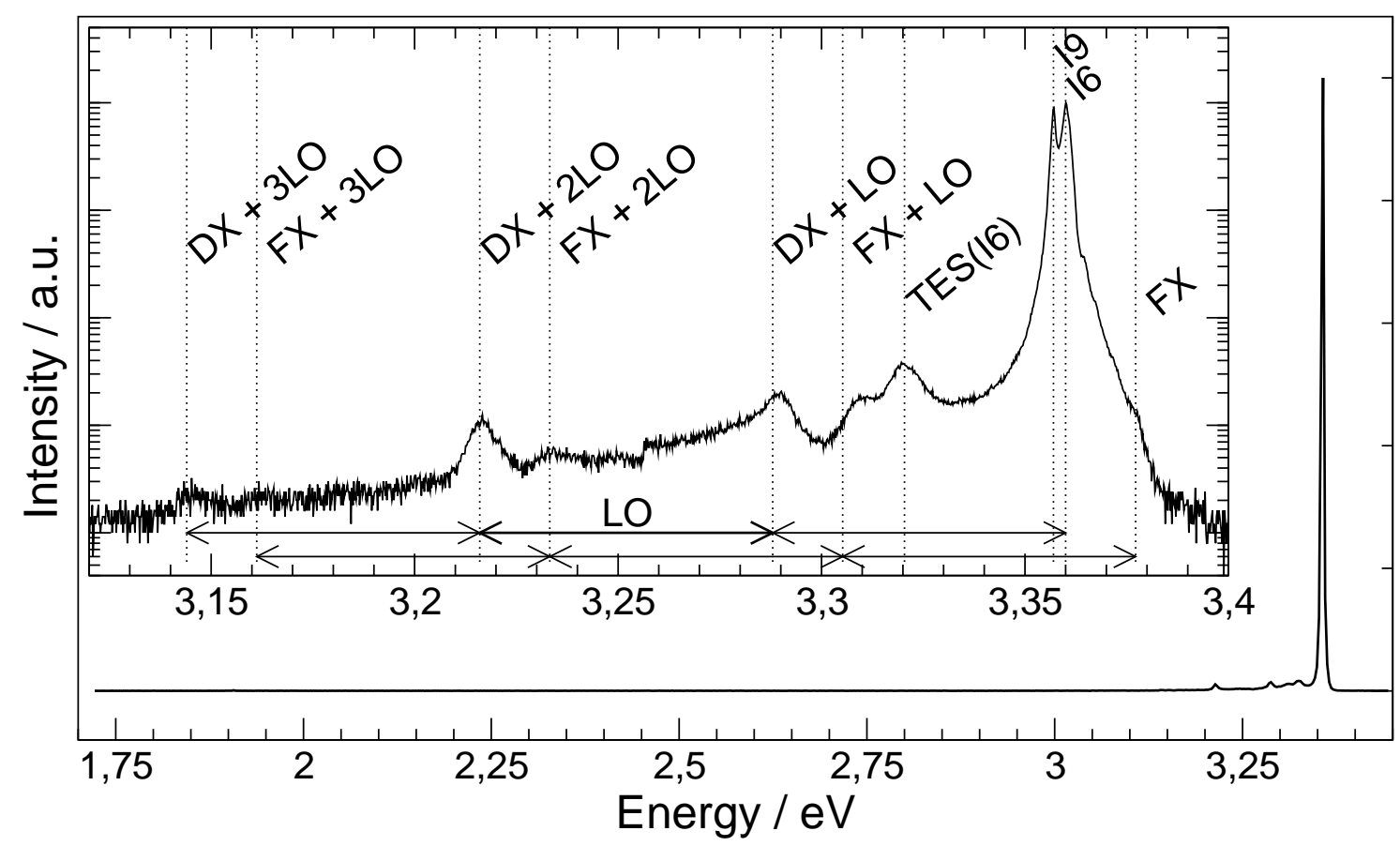

Abbildung 3.3: Lumineszenz von Zinkoxid-Nanodrähten bei tiefer Temperatur. Das Übersichtsspektrum zeigt ausschließlich Lumineszenz im bandkantennahen Bereich. Der Einsatz zeigt die bandkantennahe Lumineszenz von Zinkoxid-Nanodrähten auf logarithmischer Skala. Die Spektren wurde bei einer Probentemperatur von $14 \mathrm{~K}$ unter moderater Anregung bei 3,815 eV aufgenommen. Die einzelnen Linien können ihrer energetischen Lage nach dem freien Exziton (FX), den donatorgebundenen Exzitonen I6 und I9, dem zwei-Elektronen-Übergang von I6 (TES) und Phononenwiederholer zugeordnet werden.

Zustände geprägt ist. Lumineszenz tiefer Störstellen ist nicht zu beobachten. Dies ist ein erstes Zeichen hoher optischer Qualität der Nanodrähte.

Der Einsatz in Abbildung 3.3 zeigt den Ausschnitt des bandkantennahen Energiebereichs des Lumineszenzspektrums auf einer logarithmischen Skala. Das Spektrum wird von zwei scharfen Linien bei 3,360 eV und 3,357 eV dominiert. Diese werden in der Literatur neutralen, donatorgebundenen Exzitonen $\left(\mathrm{D}^{0} \mathrm{X}\right)$ zugeordnet und mit I6 und I9 bezeichnet [Mey04]. Mit etwa zwei bis drei Größenordnungen geringerer Intensität wird eine Linie bei 3,320 eV beobachtet, die einem zwei-ElektronenÜbergang von I6 (TES) zugeordnet wird. Dabei verbleibt das Elektron des Donatorniveaus im angeregten Zustand. Die TES-Linie tritt folglich um die Anregungsenergie zu niedrigen Energien verschoben auf. Außerdem ist an der hochenergetischen 
Flanke der I6-Linie eine ausgeprägte Schulter zu erkennen, deren energetische Position der des freien Exzitons (FX) entspricht (3,3772 eV). Mit dem Abstand der Energie longitudinal optischer Phononen (LO, $72 \mathrm{meV}$ ) folgen zu niedrigen Energien weitere Linien, die folglich den Phononenwiederholern des freien Exzitons zugeordnet werden. Gleiches gilt für die $\mathrm{D}^{0} \mathrm{X}$-Linien. Die LO-Phononenenergie ist in Abbildung 3.3 durch Pfeile angedeutet.

Neben den hier auftretenden Linien I6 und I9 werden in der Literatur bis zu neun weitere Linien genannt, die donatorgebundenen Exzitonen zugeordnet werden [Mey04]. Die chemische Natur der Donatoren ist weitgehend unklar. Für die Linie I6 wird Aluminium, für die Linie I8 Gallium genannt. Bei der Linie I9 konnte eindeutig gezeigt werden, dass ihre Intensität mit dem Indiumgehalt in der Probe skaliert [Mül07]. Die Breite der D ${ }^{0} \mathrm{X}$-Linien (FWHM) kann aus dem Spektrum zu etwa 2,1 meV bei der I6-Linie und 1,2 meV bei der I9-Linie entnommen werden, wobei insbesondere die I6-Linie durch eine Überlagerung mit weiteren Linien deutlich verbreitert ist. Dennoch sind diese Linienbreiten durchaus vergleichbar mit denen hochwertiger Einkristalle [Tek04].

\subsection{Temperaturabhägigkeit der Lumineszenz}

Um die Zuordnung der Linien, die im vorigen Abschnitt getroffen wurde, zu bestätigen wurden Lumineszenzmessungen durchgeführt, bei denen die Probentemperatur variiert wurde. Eine typische Temperaturserie von Lumineszenzspektren des bandkantennahen Bereichs ist in Abbildung 3.4 logarithmisch aufgetragen. Die Spektren sind auf die maximale Intensität normiert und zur besseren Übersicht entlang der Ordinate verschoben. Ausgehend von tiefen Temperaturen nimmt die Intensität der $\mathrm{D}^{0} \mathrm{X}$-Linien mit steigender Temperatur ab und verschiebt spektral zu niedriger Energie. Bei einer Temperatur von $120 \mathrm{~K}$ ist nahezu keine Lumineszenz donatorgebundener Exzitonen mehr zu beobachten. Simultan nimmt auch die Intensität des zwei-Elektronen-Übergangs sowie der Phononenwiederholer der $\mathrm{D}^{0} \mathrm{X}$ Linien ab. Die Lokalisierungsenergie der Exzitonen $E_{l o c}$ an den Donatoren ist gegeben durch $E_{l o c}=E_{F X}-E_{D^{0} X}$ und beträgt damit für die intensivste Linie (I6) $\approx 19 \mathrm{meV}(\approx 115 \mathrm{~K})$ [Mey04]; entsprechend lässt sich die abnehmende Intensität der $\mathrm{D}^{0} \mathrm{X}$-Linien mit steigender Temperatur darauf zurückführen, dass die Exziton- 


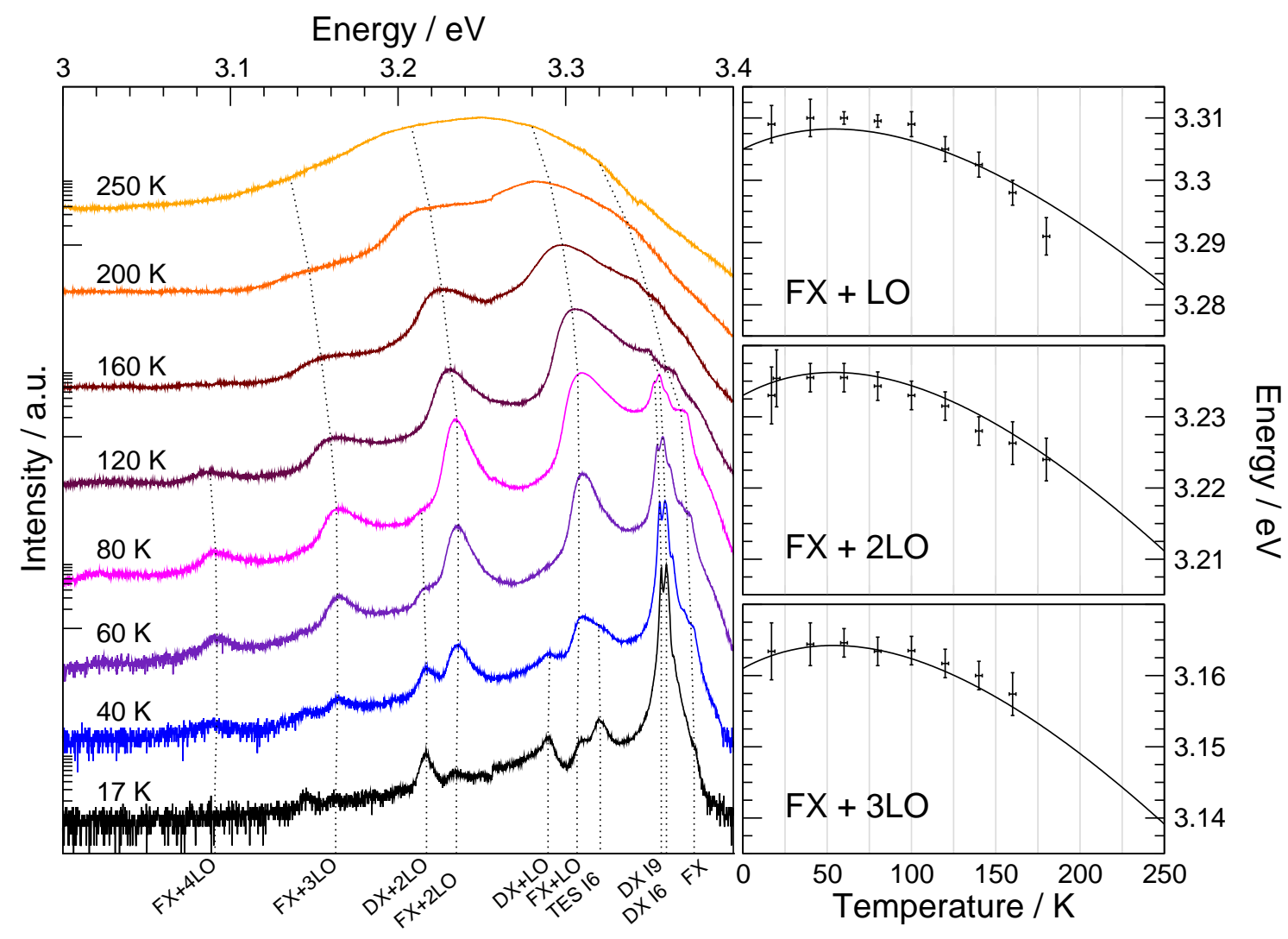

Abbildung 3.4: Temperaturabhängigkeit der Lumineszenz von Zinkoxid-Nanodrähten. Die Spektren wurden bei den angegebenen Probentemperaturen unter moderater Anregung bei 3,815 eV aufgenommen. Im rechten Teil sind die Positionen der Phononenwiederholer als Funktion der nominellen Probentemperatur aufgetragen. Die durchgezogenen Linien sind Anpassungen mit dem Varshnimodell.

Donatorkomplexe bei steigender Temperatur thermisch aktiviert dissozieren.

Während die $\mathrm{D}^{0} \mathrm{X}$-Lumineszenz mit steigender Temperatur an relativer Intensität verliert, steigt die Intensität der FX-Linie sowie die der FX-Phononenwiederholer. Bei $250 \mathrm{~K}$ ist das Spektrum typischerweise durch die FX-Linie und dessen ersten beiden Phononenwiederholer geprägt [Vos06]. Analog zu den gebundenen Exzitonen schiebt auch die FX-Lumineszenz mit steigender Temperatur spektral zu niedrigeren Energien.

Die Ursache der spektralen Verschiebung gebundener und freier Exzitonen ist mit der Temperaturabhängigkeit der Bandlücke zu verstehen. Die Bandlücke eines Halbleiters steht in einem nicht linearen Zusammenhang mit der Temperatur [Hau06c]. 
Dies liegt unter anderem darin begründet, dass auch die thermische Ausdehnung der Einheitszelle eine nicht lineare Funktion der Temperatur ist [Var67]. Die Temperaturabhängigkeit der Bandlücke kann semiempirisch mit der Varshni-Gleichung beschrieben werden (vgl. Gleichung 3.2) [Var67].

$$
E_{\text {gap }}(T)=E(0)-\frac{\alpha T^{2}}{\beta+T}
$$

Dabei bezeichnet $E(0)$ die Bandlücke bei $0 \mathrm{~K}$ und $T$ die Temperatur; $\alpha$ und $\beta$ sind empirische, materialabhängige Parameter.

Die energetische Lage des freien Exzitons ist durch die Differenz von temperaturabhängiger Bandlücke (Gleichung 3.2) und Exzitonbindungsenergie $(\approx 60 \mathrm{meV})$ gegeben. Das freie Exziton ist in Abbildung 3.4 lediglich als Schulter zu erkennen, die Phononenwiederholer hingegen sind als deutliche Linien erkennbar. Folglich wurde die Lage der Maxima der Phononenrepliken gewählt, um auf die energetische Lage der freien Exzitonen zu schließen. Die Lage des Maximums $E_{L O}^{\max }(n, T)$ der n-ten Phononenrepliken ist gegeben durch [Kli75]:

$$
E_{L O}^{\max }(n, T)=E_{g a p}(T)+\frac{3}{2} k_{b} T-E_{b}^{E x}-n \hbar \omega(L O) .
$$

Dabei bezeichnet $E_{g a p}(T)$ die temperaturabhängige Bandlücke, $k_{b}$ die Boltzmannkonstante, $E_{b}^{E x}$ die Exzitonbindungsenergie und $\hbar \omega(L O)$ die Energie longitudinal optischer Phononen. Im rechten Teil der Abbildung 3.4 ist die energetische Lage der ersten drei Phononenrepliken des freien Exzitons, wie sie den einzelnen Spektren entnommen wurde, als Funktion der Temperatur aufgetragen. Der Fehler in der Temperatur ist messtechnisch bedingt, der Fehler in der Energie ein Ablesefehler. Die durchgezogene Linie ist eine simultane Angleichung der Messwerte mit Gleichung 3.3 an die Messwerte. Dabei wurden die empirischen Faktoren $\alpha\left(=0,52 \cdot 10^{-3} \mathrm{eV} / \mathrm{K}\right)$ und $\beta(=350 \mathrm{~K})$ angeglichen, alle übrigen Parameter sind fest. Die angepassten Werte sind in guter Übereinstimmung mit der Literatur [Pri04, Wis07]. Die mit den angepassten Werten für $\alpha$ und $\beta$ berechneten Positionen der Maxima der Phononenrepliken und des freien Exzitons sind im linken Teil der Abbildung als gestrichelte Linien in die Lumineszenzspektren eingetragen. 


\subsection{Lumineszenzuntersuchungen an einzelnen Nanodrähten}

Die Lumineszenzuntersuchungen in den vorigen Abschnitten wurden an Nanodrahtensemblen durchgeführt. Wie das SEM-Bild in Abbildung 3.2 a) zeigt, befinden sich unter den Nanodrähten oft mikrokristalline Strukturen. Wird an die Verwendung der Nanodrähte als funktionelle Bausteine gedacht, so sind die Eigenschaften einzelner Nanodrähte von Interesse. Inwiefern sich die optischen Eigenschaften einzelner Nanodrähte von denen eines Ensembles unterscheiden, soll in diesem Abschnitt untersucht werden.

Die Lumineszenzmessungen an einzelnen Nanodrähten wurden in einem $\mu$-PL Aufbau an der Universität Bremen durchgeführt. Der Strahldurchmesser beträgt dort $\approx 2 \mu \mathrm{m}$. Um einzelne Nanodrähte zu untersuchen, müssen die Drähte folglich vereinzelt werden. Dazu wurden, wie im Abschnitt 2.2.2 beschrieben, einige Nanodrähte auf ein sauberes Substrat übertragen. Abbildung 3.5 zeigt die Lumineszenz einzelner Nanodrähte im Vergleich mit der Lumineszenz der gesamten Probe. Der Vergleich der Spektren zeigt keine wesentlichen Unterschiede. So werden alle Spektren von den donatorgebundenen Exzitonen dominiert. Die Linien I6 und I9, die das Ensemblespektrum dominieren, sind auch in den Spektren der einzelnen Drähte dominant. In den Spektren einzelner Drähte können bis zu fünf Donatorkomplexe identifiziert werden. Es zeigt sich, dass die entsprechenden Linien bei den Einzeldrahtspektren zum Teil unterschiedlich intensiv ausgeprägt sind, was bei dem Ensemblespektrum zu einem strukturlosen Untergrund zwischen der I6-Linie und dem freien Exziton (FX) führt. Die FX-Bande ist bei dem Spektrum des Ensembles etwas deutlicher ausgeprägt, was vermutlich auf die höhere Probentemperatur zurückzuführen ist. In dem niederenergetischen Teil des Spektrums sind sowohl bei den einzelnen Nanodrähten, als auch beim Ensemble die Phononenrepliken der gebundenen Exzitonen relativ schwach zu erkennen. Bei 3,333 eV ist in dem Spektrum des Nanodrahtensembles eine scharfe Linie zu erkennen, die in der Literatur als YLinie bezeichnet und im Zusammenhang mit strukturellen Defekten beobachtet wird [Mey04]. Diese tritt in den Spektren einzelner Drähte nicht auf, was wiederum ein Hinweis für die hohe Qualität der Nanodrähte ist.

Das Spektrum von Nanodraht 1 unterscheidet sich relativ deutlich von dem der 


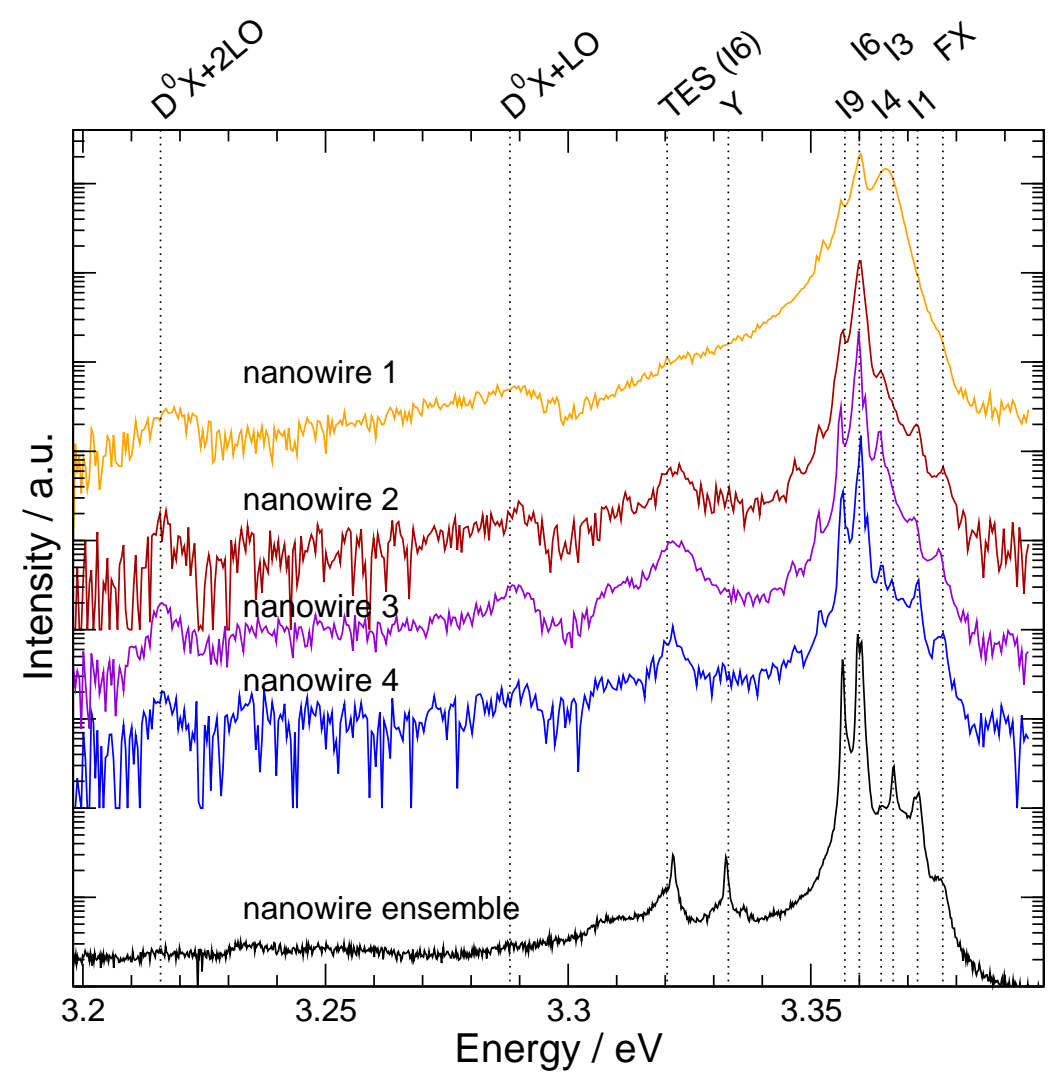

Abbildung 3.5: Lumineszenz von Zinkoxid-Nanodrahtensemblen im Vergleich zur Lumineszenz einzelner Drähte. Die Lumineszenz des Ensembles wurde bei $15 \mathrm{~K}$, die der einzelnen Drähte bei $4 \mathrm{~K}$ unter einer Anregung bei 3,815 eV gemessen.

weiteren Nanodrähte. Im Bereich der mit I3 gekennzeichneten Bande tritt bei diesem Draht eine intensive Bande mit vergleichsweise großer Halbwertsbreite auf. Diese wurde in der Literatur mit der Oberfläche von Zinkoxid in Zusammenhang gebracht, was im folgenden Abschnitt näher diskutiert werden soll.

Insgesamt zeigt der Vergleich der Photolumineszenzspektren von einzelnen Drähten mit dem Ensemble, dass die Messungen am Ensemble die wesentlichen Strukturen wiedergeben. Insofern ist es gerechtfertigt, die optischen Eigenschaften des Ensembles als Eigenschaft der Nanodrähte zu bezeichnen. Dies zeigt zudem, dass die Eigenschaften der Drähte relativ homogen sind, was ebenso in der Literatur beschrieben wird [Wis06a]. 

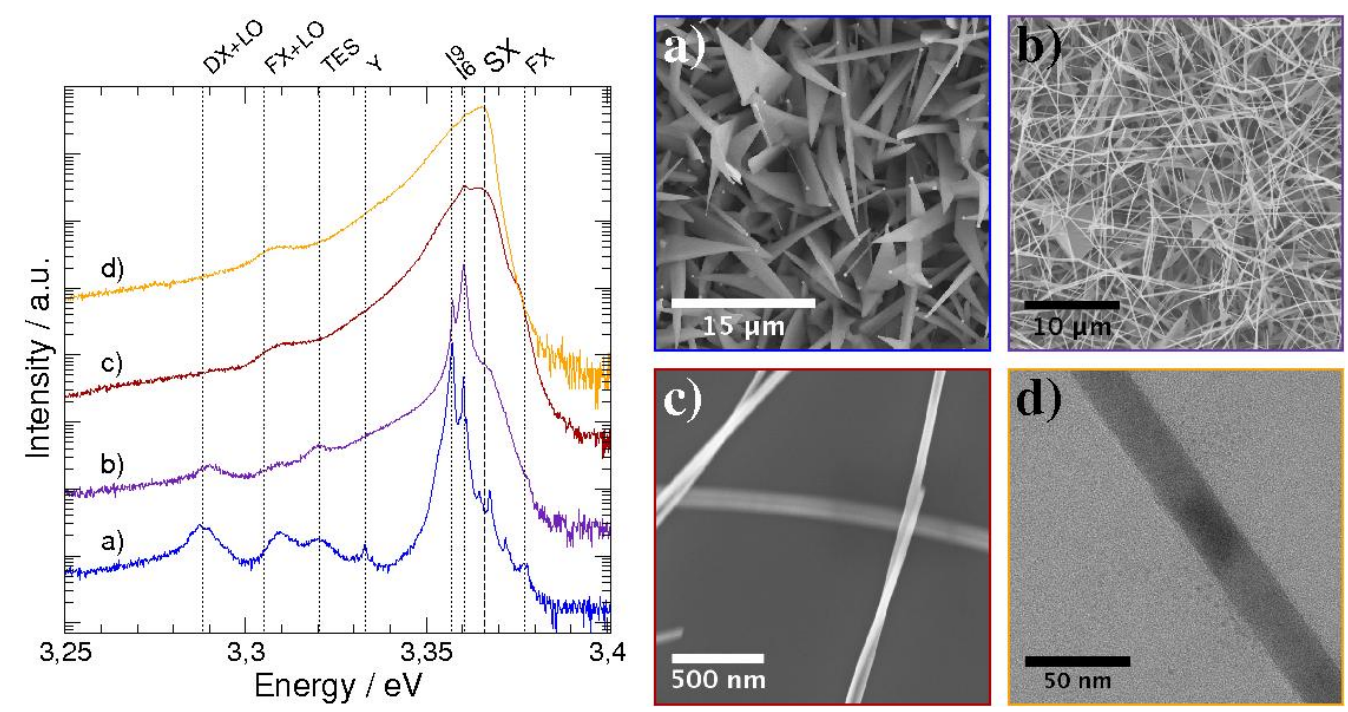

Abbildung 3.6: Lumineszenz von Zinkoxid-Nanostrukturen unterschiedlicher Größe. Die Spektren wurden bei einer Temperatur von $15 \mathrm{~K}$ unter Anregung bei 3,815 eV(cw) aufgenommen. Die Lumineszenz der Nanostrukturen im Bereich der Bandkante ändert sich stark, wenn die Strukturgröße verändert wird. Insbesondere die SX-Bande bei 3,366 eV wird bei kleiner werdenden Strukturen zunehmend dominant. Die SEM- und TEM-Bilder zeigen die untersuchten Strukturen.

\subsection{Einfluss der Oberfläche}

Ein Vergleich der Lumineszenzeigenschaften von Zinkoxid-Nanodrähten, wie sie in den vorigen Abschnitten erläutert wurden, mit der Lumineszenz von ZinkoxidVolumenmaterial, wie sie in der Literatur dargestellt ist [Mey04], zeigt im Wesentlichen keine Unterschiede. Dies ist auch nicht erstaunlich, denn der Exzitonbohrradius in Zinkoxid beträgt etwa 1,8 bis $2 \mathrm{~nm}$ [LaB99], während der Durchmesser der Nanodrähte im Bereich 50 bis $200 \mathrm{~nm}$ liegt.

Ein erster Hinweis auf eine Abweichung zeigt das Spektrum von Nanodraht 1 in Abbildung 3.5. Hier tritt eine breite Bande im Bereich der donatorgebundenen Exzitonen bei 3,366 eV auf. In der Literatur wird diese Bande mit der Oberfläche von Zinkoxid in Verbindung gebracht [Tra90, Gra05]. Um diesen Zusammenhang zu untersuchen, wurden Lumineszenzmessungen an Zinkoxid-Strukturen unterschiedlicher Größe durchgeführt, bei denen sich das Oberflächen-Volumen-Verhältnis entsprechend ändert [Sti07]. 
Abbildung 3.6 zeigt Lumineszenzspektren unterschiedlicher Nanostrukturen, die bei einer nominellen Probentemperatur von $15 \mathrm{~K}$ und bei moderater Anregung bei 3,815 eV aufgenommen wurden. Die Spektren sind normiert und zur besseren Übersicht entlang der Ordinate verschoben aufgetragen. Die hier gezeigten Spektren spiegeln ein charakteristisches Verhalten wider, das an einer Vielzahl von untersuchten Proben beobachtet wurde. Es wurden hier explizit Proben ausgewählt, die auf unterschiedliche Weise in verschiedenen Gruppen hergestellt wurden. Elektronenmikroskopische Aufnahmen der untersuchten Proben sind im rechten Teil von Abbildung 3.6 dargestellt. So zeigen Abbildung a) und b) SEM-Aufnahmen von Proben, die in Göttingen hergestellt wurden (vgl. Abschnitt 2.1.3). Bei Probe a) wurde eine etwas dickere Goldschicht auf das Wachstumssubstrat aufgedampft. Die Strukturen haben eine bandartige, dreieckige Morphologie. Die Dicke der Strukturen beträgt einige $100 \mathrm{~nm}$, die Fläche etwa $5 \times 10 \mu \mathrm{m}$. Am Ende der Bänder befindet sich der Goldkatalysator. Probe b) ist ein typisches Nanodrahtensemble. Die Nanodrähte haben einen mittleren Durchmesser von $\approx 150 \mathrm{~nm}$ und eine Länge von 5 - $10 \mu \mathrm{m}$. Probe c) wurde in der Gruppe Li/Hong Kong hergestellt und zeigt Drähte mit einem relativ monodispersem Durchmesser von $\approx 40 \mathrm{~nm}$. Die Drähte wurden vor der Messung vom Wachstumssubstrat entfernt und auf ein sauberes Siliziumsubstrat übertragen. Eine TEM-Aufnahme von Probe d) zeigt einen Zinkoxid-Nanodraht, der in der Gruppe Lu/UCI hergestellt wurde. Der Durchmesser dieser Drähte beträgt in etwa 10 - 30 nm. Die Drähte wurden mit Zinn als Katalysator bei niedrigen Temperaturen synthetisiert (vgl. Abschnitt 2.1.4).

Das Lumineszenzspektrum von Probe a) zeigt die typischen Strukturen, die in Abschnitt 3.1 dem freien Exziton (FX) und Komplexen gebundener Exzitonen zugeordnet wurden. Im Unterschied zu diesem Spektrum zeigt das Spektrum von Probe b) im Bereich des SX keine scharfen Linien mehr. Dort findet sich ein breiter Untergrund. Das Spektrum von Probe c) wird bereits von der SX-Linie dominiert. Die Linie hat eine deutlich asymmetrische Form, die zu hoher Energie steil abfällt und zu niedriger Energie inhomogen verbreitert ist. An der Flanke der SX-Linie zu hoher Energie findet sich eine Schulter, die dem freien Exziton zugeordnet werden kann. $\mathrm{Zu}$ niedrigen Energien ist auf der SX-Bande noch schwach die I6-Linie zu erkennen, die im Spektrum der Probe d) nicht mehr zu beobachten ist. Bei Probe d) lässt sich auch das FX nicht beobachten, allerdings dessen Phononenwiederholer. 
Die SX-Bande wurde zunächst an in Vakuum gespaltenen Zinkoxid-Einkristallen [Tra90], später an Zinkoxid-Nanodrähten beobachtet [Gra05, Wis06b]. Eine Untersuchung an einzelnen Nanodrähten unterschiedlichen Durchmessers d zeigte, dass das Intensitätsverhältnis zwischen SX-Bande und den Banden übriger gebundener Exzitonen einer 1/d Abhängigkeit folgt, was das Oberflächen zu Volumenverhältnis wiederspiegelt [Vos08]. Dies ist bereits ein gutes Indiz für die Identifizierung der SX-Bande als Oberflächeneffekt. Ein weiteres Indiz wurde in der Linienform der SX-Bande gesucht. Analog zu Untersuchungen an oberflächengebundenen Exzitonen in CdS wurde zunächst vermutet, dass die Ursache der zu niedrigen Energien inhomogen verbreiterten Linienform ein variierender Abstand eines Exzitonkomplexes zur Oberfläche ist [Tra90]. Messungen von Transienten an unterschiedlichen spektralen Positionen in der SX-Bande zeigten allerdings deutlich verschiedene Lebenszeiten, die sich mit einem Exzitonkomplex nicht erklären lassen [Wis06b]. Entsprechend wird die typische Linienform auf lokalisierte Exzitonen nahe der Oberfläche zurückgeführt, wobei unterschiedliche lokale Umgebungen (z.B. ungebundene Zustände, Adatome, Rekonstruktionen, etc.) zu einer Vielzahl schwach lokalisierter exzitonischer Zustände führt. Durch die schwache Lokalisierung der Exzitonen von $\approx 9 \mathrm{meV}$ [Wis06b] dissoziert der Exzitonkomplex ab etwa $50 \mathrm{~K}$; eine SXBande wird im Photolumineszenzspektrum nicht weiter beobachtet. Entsprechend dieser Argumentation ist es sinnvoll anzunehmen, dass die Exzitonen nicht an der Oberfläche selbst, sondern vielmehr in eine Mantelschicht lokalisieren, die somit Ursprung der SX-Bande ist. Mit Hilfe eines zylindrischen Modells [Sha04] konnte in Ref. [Vos08] aus dem oben beschriebenen Intensitätsverhältnis von SX- und übrigen BEC-Banden eine Dicke k der Mantelschicht bestimmt werden, die im Bereich $0 \mathrm{~nm}<k<25 \mathrm{~nm}$ liegt. Wird dieses Ergebnis mit den Spektren in Abbildung 3.6 verglichen, so sollte Probe c) mit einem Drahtdurchmesser von $\approx 40 \mathrm{~nm}$ gerade nur noch SX-Lumineszenz zeigen. Das entsprechende Spektrum wird wie erwartet von der SX-Bande dominiert, zeigt allerdings mit sehr geringer Intensität auch die I6-Linie. Dies bestätigt, dass der Radius des Drahtes sehr nah an der Dicke der Mantelschicht liegt. Bei den dünneren Drähten der Probe d) wird dann tatsächlich ausschließlich SX-Lumineszenz beobachtet. 


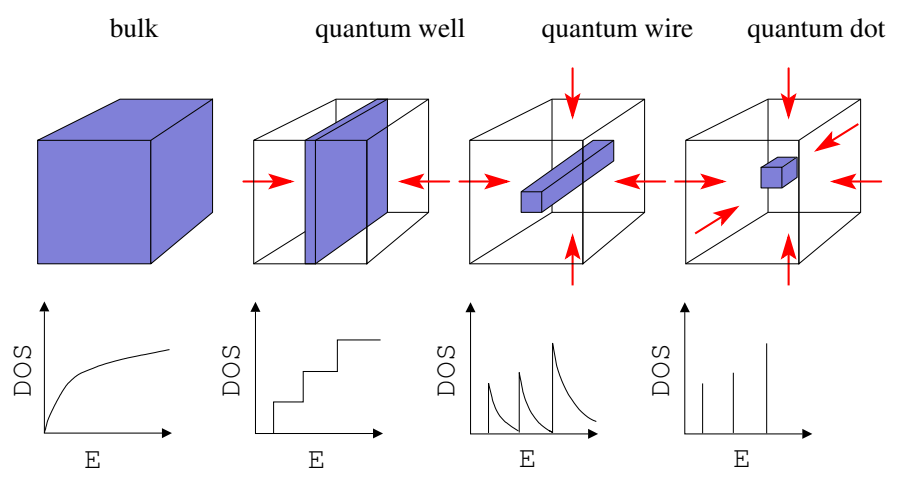

Abbildung 3.7: Reduzierung der Dimensionalität vom Volumenkristall (bulk) zum Quantenpunkt (quantum dot) mit der entsprechenden Diskretisierung der Zustandsdichte (DOS) in ihren Energiewerten nach [Pro00].

\subsection{Quantum confinement in ultra-dünnen Zinkoxid- Nanodrähten}

Eindimensionale Halbleiter können andere optische Eigenschaften als Volumenkristalle haben. Durch die Einschränkung der Elektronenbewegung auf eine Dimension kommt es zu einer charakteristischen Veränderung der Zustandsdichte [Kay88] (siehe Abbildung 3.7). Dieser Effekt wird als Größenquantisierung oder quantum confinement bezeichnet. Dieser Effekt tritt auf, wenn die Strukturgröße in den Bereich des Exzitonbohrradius gelangt. Die Strukturbegrenzung wirkt dabei wie ein beschränkendes Potential, durch das eine Quantisierung der Zustände und eine Vergrößerung der Bandlücke erfolgt. Der Exzitonbohrradius in Zinkoxid beträgt etwa 1,8 bis 2 nm [LaB99]. Entsprechend werden Quantisierungseffekte erst bei ZinkoxidNanodrähten mit sehr geringem Durchmesser erwartet. Im Folgenden werden Untersuchungen an Zinkoxid-Nanodrähten gezeigt, deren mittlerer Durchmesser $\approx 4 \mathrm{~nm}$ beträgt [Sti07].

Die Nanodrähte wurden in einem VLS-basierten Prozess in der Kollaboration Lu/UCI hergestellt, wie er in Abschnitt 2.1.4 beschrieben ist. Dabei wurde metallisches Zink als Ausgangsmaterial und eine $5 \mathrm{~nm}$ dicke Zinnschicht auf Siliziumsubstraten als Katalysator verwendet. Nach einer Wachstumszeit von einer Stunde bei $500{ }^{\circ} \mathrm{C}$ konnte ein grauer Niederschlag auf den Substraten beobachtet werden. Die Morphologie der Probe wurde im SEM untersucht. Auf dem SEM-Bild in Ab- 

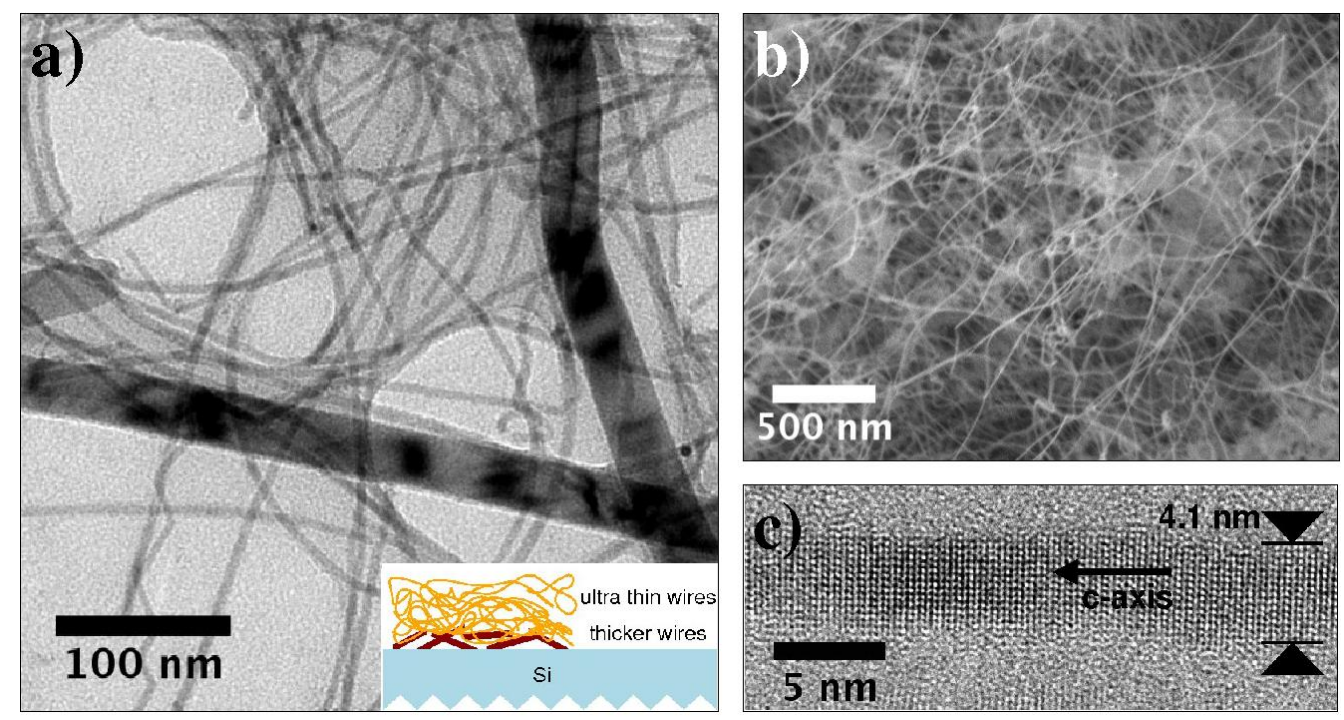

Abbildung 3.8: Morphologie von ultradünnen Nanodrähten. a) TEM-Hellfeldaufnahme bei geringer Vergrößerung. Es sind sowohl Nanodrähte mit einem mittleren Durchmesser von $\approx 30 \mathrm{~nm}$, als auch ultradünne Drähte mit einem Durchmesser von $\approx 4 \mathrm{~nm}$ zu erkennen. b) SEM-Aufnahme des Nanodrahtensembles. Die Länge der Drähte beträgt einige Mikrometer. Unter den Drähten sind helle Bereiche erkennbar. c) HRTEM-Aufnahme eines einzelnen ultradünnen Nanodrahtes. Die Aufnahme bestätigt die hohe Kristallinität der Probe. Die Drahtachse verläuft entlang der kristallographischen c-Achse.

bildung 3.8 b) ist eine dichte Verteilung von Nanodrähten zu erkennen, die keine präferenzielle Ausrichtung haben. Die typische Länge der Drähte beträgt einige Mikrometer, während der Durchmesser der Drähte kleiner als $20 \mathrm{~nm}$ ist. Eine genaue Bestimmung des Durchmessers ist im SEM nicht möglich, da die freistehenden Drähte durch Aufladung zu schwingen beginnen. Desweiteren sind in dem SEM-Bild größere, helle Bereiche diffus durch die Nanodrähte hindurch erkennbar. Aufgrund ähnlicher Beobachtungen wurde in [Wan04a] ein Wachstumsmodell vorgeschlagen, bei dem, wie in Abschnitt 2.1.2 beschrieben, der Zinn-Katalysator auf dem Substrat zurückbleibt und die Drähte aus der Legierung herauswachsen. Die hellen Bereiche wurden Zinn-Inseln auf dem Substrat zugeordnet. Dieses Modell wird durch die Beobachtung gestützt, dass weder an den Nanodrähten in [Wan04a] noch an diesen Drähten Legierungskugeln beobachtet werden konnten, wie es nach einem typischen VLS-Mechanismus zu erwarten ist.

Um weiteren Aufschluss über die Größenverteilung und die Kristallinität der 
Nanodrähte zu bekommen, wurden TEM-Untersuchungen durchgeführt. Dazu wurde zunächst eine Suspension aus Nanodrähten und Isopropanol erstellt (vgl. Abschnitt 2.2.2), die dann auf ein TEM-Netz gegeben wurde. Abbildung 3.8 a) zeigt eine TEM-Hellfeldaufnahme mit niedriger Vergrößerung. $\mathrm{Zu}$ erkennen sind sowohl Nanodrähte mit einem mittleren Durchmesser von $\approx 4 \mathrm{~nm}$, als auch dickere Nanodrähte mit einem Durchmesser von $\approx 30 \mathrm{~nm}$. In dem Bereich dazwischen konnten keine Nanodrähte beobachtet werden. Da die dickeren Nanodrähte auf dem SEM-Bild in Abbildung 3.8 b) nicht zu erkennen sind, ist anzunehmen, dass sie sich auf der Orginalprobe unter den dünnen Nanodrähten befunden haben, wie es in der Schemazeichnung im Einsatz von Abbildung 3.8 a) angedeutet ist.

Abbildung 3.8 c) zeigt eine HRTEM-Aufnahme eines ultradünnen Nanodrahtes. Durch Ausmessen der atomaren Ebenenabstände lässt sich die Drahtachse der kristallographischen c-Achse zuordnen. Es können nur wenige Kristalldefekte in Form von Stapelfehlern beobachtet werden. Dies bestätigt erneut die hohe Qualität selbst dieser ultradünnen Nanodrähte. Durch Ausmessen mehrerer Nanodrähte konnte ein mittlerer Durchmesser von 4 - 5 nm ermittelt werden.

Um die optischen Eigenschaften der ultradünnen Drähte zu untersuchen, wurden PL-Untersuchungen an der gewachsenen Probe durchgeführt, auf der sich allerdings, wie oben beschrieben, auch dickere Nanodrähte $(\approx 30 \mathrm{~nm})$ befanden. Entsprechend wird erwartet, dass auch das Lumineszenzspektrum Eigenschaften der ultradünnen und dickeren Drähte zeigt. Die Lumineszenzspektren wurden unter moderater Anregung der Probe bei 3,815 eV aufgenommen. Abbildung 3.9 zeigt ein logarithmisch aufgetragenes Übersichtsspektrum, das bei einer nominellen Probentemperatur von $15 \mathrm{~K}$ gemessen wurde. Das Spektrum wird von einer asymmetrischen Bande bei 3,366 eV dominiert, die in Abschnitt 3.4 einem nahe der Oberfläche lokalisiertem Exziton zugeordnet wurde und als SX bezeichnet wird. Außerdem wird im Bereich der Bandkante eine Bande bei 3,327 eV beobachtet, auf die zu niedriger Energie Phononenwiederholer folgen. Der Abstand der Phononenwiederholer beträgt, wie in Volumenmaterial und dickeren Nanodrähten für longitudinal optische (LO) Phononen beobachtet, $72 \mathrm{meV}$. Eine größenbedingte Änderung der Phononenenergie kann nicht beobachtet werden [Sen03]. Die grüne Lumineszenzbande bei 2,6 eV, die in der Literatur meist Sauerstoffvakanzen zugeordnet wird [Lei01], ist sehr schwach ausgeprägt. Die Beobachtung, dass diese Bande bei kleineren Strukturen stärker 


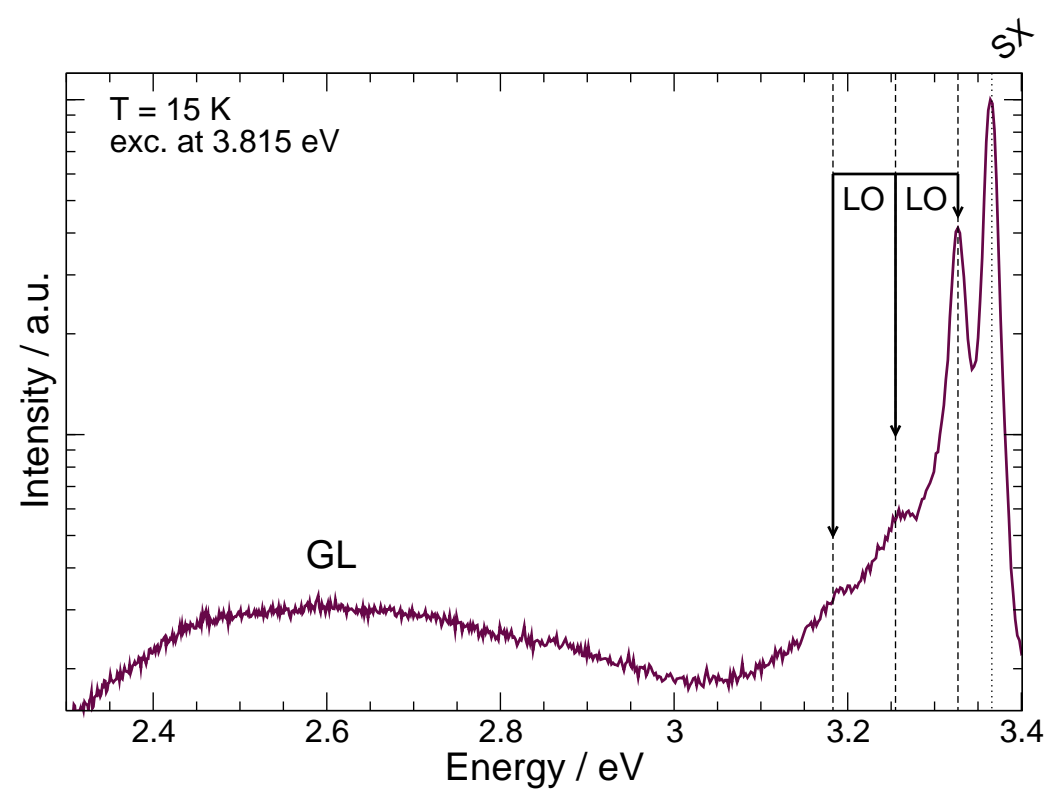

Abbildung 3.9: Übersichts-Photolumineszenzspektren von ultradünnen Zinkoxid-Nanodrähten bei tiefer Temperatur. Das Spektrum zeigt neben der charakteristischen SX-Bande eine Lumineszenz bei 3,327 eV sowie dazu passende Phononenwiederholer. Die grüne Lumineszenz (GL) ist nur schwach ausgeprägt.

ausgeprägt ist und in Zusammenhang mit der Oberfläche auftritt [Sha04], bestätigt sich hier nicht. Da die untersuchten Strukturen freistehend und besonders dünn sind, kann die Wärmedissipation stark eingeschränkt sein. Dies kann durchaus zu einer lokalen Erwärmung der Nanodrähte durch die Laseranregung führen. Entsprechend können lokal deutlich andere Temperaturen herrschen als auf dem Probenträger gemessen werden.

Um insbesondere die Lumineszenzbande bei 3,327 eV besser $\mathrm{zu}$ verstehen, ist in Abbildung 3.10 a) der bandkantennahe Energiebereich des Spektrums zusammen mit dem Spektrum eines Nanodrahtensembles mit größerem mittleren Durchmesser von $\approx 10 \mathrm{~nm}$ (vgl. Abschnitt 3.4) aufgetragen. Die Spektren wurden bei einer nominellen Probentemperatur von $13 \mathrm{~K}$ aufgenommen. Beide Spektren werden von der SX-Bande dominiert, die bei dem Spektrum der ultradünnen Drähte eine Schulter an der hochenergetischen Flanke hat. Das Spektrum der Nanodrähte mit $\approx 10 \mathrm{~nm}$ Durchmesser zeigt außerdem eine Bande bei 3,308 eV, die der ersten Phononenreplik des freien Exzitons zugeordnet wird. Mit Gleichung 3.3 berechnet sich die 

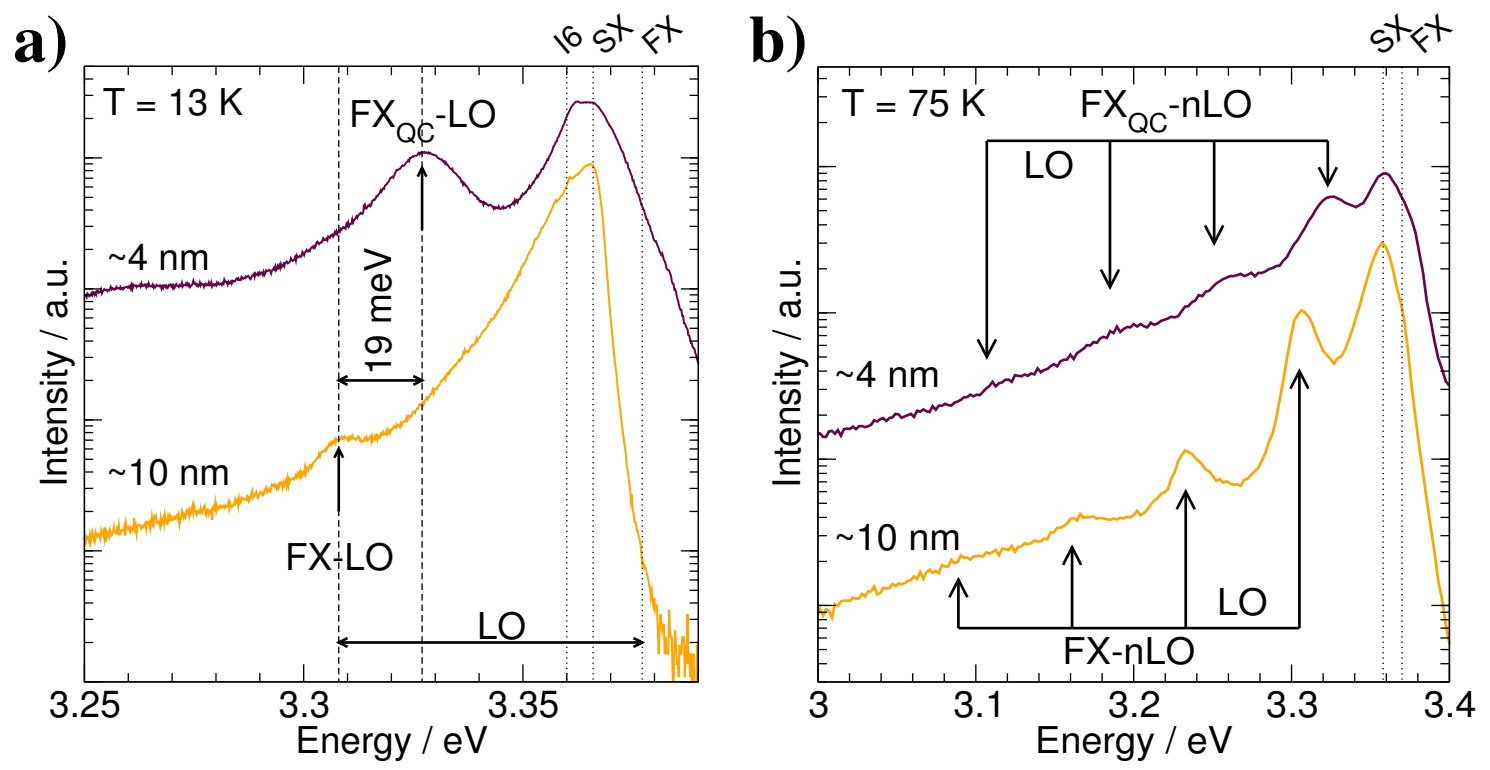

Abbildung 3.10: Vergleich von Photolumineszenzspektren von ultradünnen ZinkoxidNanodrähten mit Drähten etwas größeren Durchmessers. a) Die PL im bandkantennahen Bereich wird bei beiden Drahtensemblen von der SX-Bande geprägt. Der Phononenwiederholer des freien Exzitons (FX-LO) tritt bei den dünnen Drähten nicht auf, dafür aber eine Bande bei $3,327 \mathrm{eV}$ $\left(F X_{Q C}-L O\right)$, die ähnliche Form hat. b) Bei etwas höherer Temperatur $(75 \mathrm{~K})$ zeigt sich, dass sowohl die FX-LO-Bande als auch die $F X_{Q C}-L O$ Bande ausgeprägte Phononenwiederholer haben.

Energie des freien Exzitons zu 3,378 eV, was in guter Übereinstimmung mit Werten von Volumenkristallen ist [Mey04]. Eine Bande bei 3,308 eV tritt im Spektrum der ultradünnen Nanodrähte nicht auf, dafür eine Bande bei 3,327 eV, die ähnliche Form hat und ebenso charakteristisch Phononenwiederholer zeigt. Dies wird insbesondere bei etwas höheren Temperaturen deutlich, wie ein Vergleich der Spektren in Abbildung 3.10 b) zeigt, die bei einer Probentemperatur von $75 \mathrm{~K}$ aufgenommen wurden. Diese Ähnlichkeit der Banden legt die Vermutung nahe, dass es sich bei der Bande bei 3,327 eV um einen Phononenwiederholer eines blauverschobenen freien Exzitons handelt.

Um diese Vermutung weiter zu bestätigen wurden Lumineszenzspektren des bandkantennahen Energiebereichs bei unterschiedlichen Probentemperaturen aufgenommen. In Abbildung 3.11 a) sind diese Spektren normiert und auf einer logarithmischen Skala entlang der Ordinate verschoben aufgetragen. Aus der charakteris- 

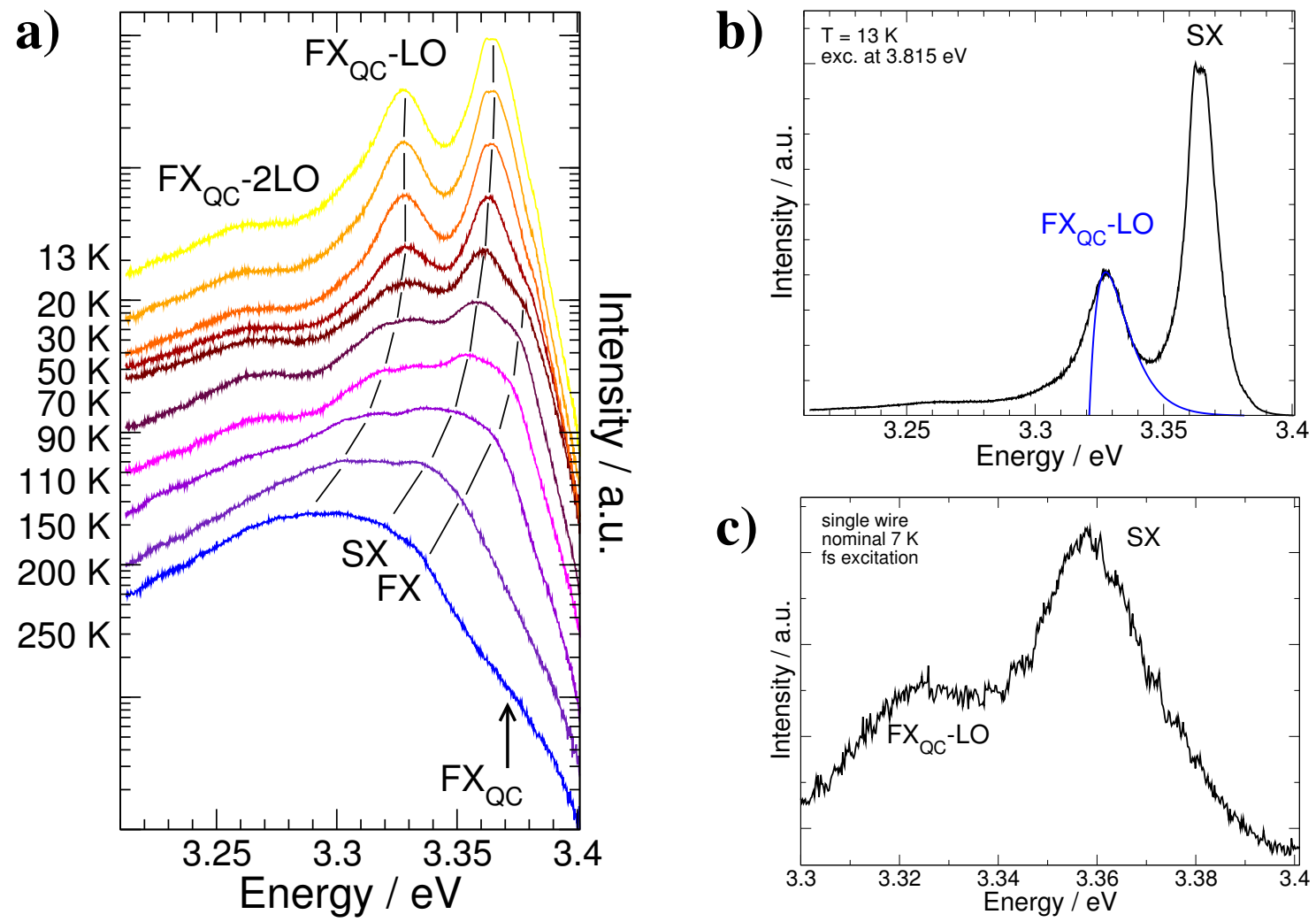

Abbildung 3.11: a) Temperaturabhängigkeit der Lumineszenz ultradünner Nanodrähte im bandkantennahen Bereich. b) Numerische Anpassung der Linienform des Phononenwiederholers. c) Mikrophotolumineszenzspektrum eines einzelnen ultradünnen Nanodrahtes.

tischen Veränderung der Spektren mit steigender Temperatur lassen sich vier wesentliche Erkenntnisse gewinnen. (1) Die SX-Bande wird mit steigender Temperatur breiter und verschiebt zu niedriger Energie. Anders als bei dickeren Nanodrähten [Wis06b], trägt die SX-Bande auch bei $250 \mathrm{~K}$ noch zum Spektrum bei. Durch den geringen Durchmesser der Drähte lässt sich im Grunde der gesamte Draht als Oberfläche beschreiben (vgl. Abschnitt 3.4, [Vos08]), was entsprechend zu einer Reduktion der volumenartigen Zustände führt. Folglich ist auch die thermisch aktivierte Übergangswahrscheinlichkeit eines Exzitons aus einem gebundenen Zustand in den freien Zustand reduziert, was effektiv zu einer starken Erhöhung der Lokalisierungsenergie des SX-Exzitonkomplexes führt. Desweiteren führt möglicherweise das stark erhöhte Verhältnis von Oberfläche zu Volumen dazu, dass Exzitonen mit mehr als 
einem Defekt in Wechselwirkung treten, was ebenso zu einer effektiven Erhöhung der Lokalisierungsenergie führt. (2) Die Bande bei 3,327 eV wird ebenfalls mit steigender Temperatur breiter, verschiebt spektral zu niedriger Energie und ist bei $250 \mathrm{~K}$ noch im Spektrum erkennbar. Die relative Intensität der Linie steigt mit zunehmender Temperatur leicht an. Dies ist ein weiteres Indiz dafür, dass die Bande im Zusammenhang mit Phononen steht. Die energetische Lage bei 3,327 eV unterstützt die Vermutung, dass es sich bei der Bande um den ersten Phononenwiederholer des freien Exzitons handelt, das durch Quantumconfinement um $19 \mathrm{meV}$ blauverschoben auftritt. (3) Bereits bei einer Temperatur von $50 \mathrm{~K}$ wächst schwach ausgeprägt bei etwa 3,377 eV eine Schulter aus der hochenergetischen Flanke der SX-Bande. Die spektrale Position stimmt mit der Position des freien Exzitons in Zinkoxid-Volumenkristallen überein. (4) Bei hoher Temperatur (250 K) kommt bei $\approx 3,370$ eV eine weitere Schulter an der hochenergetischen Flanke der Lumineszenzbande auf. Diese spektrale Position liegt jenseits der Energie eines freien Exzitons bei dieser Temperatur und ist in Abbildung 3.11 a) entsprechend mit $\mathrm{FX}_{\mathrm{QC}}$ gekennzeichnet.

Um diese Beobachtungen zu verstehen muss berücksichtigt werden, dass sich auf der Probe neben den ultradünnen auch dickere Nanodrähte befinden, was auch das Lumineszenzspektrum widerspiegeln sollte. So lassen sich die $\mathrm{FX}_{\mathrm{QC}^{-}}$(4) und die $\mathrm{FX}_{\mathrm{QC}}$-LO-Bande (2) auf die ultradünnen Drähte, die gewöhnliche FX-Bande auf die dickeren Drähte zurückführen.

Da die $\mathrm{FX}_{\mathrm{QC}}$ Bande nur mit geringer Intensität als Schulter auftritt, lässt sich die Stärke der Blauverschiebung nicht genauer quantifizieren. Da angenommen wird, dass die Bande bei 3,327 eV der Phononenwiederholer eines blauverschobenen freien Exzitons ist wird diese Bande zur quantitativen Analyse näher untersucht, indem die Linienform mit einem Modell numerisch angeglichen wird. Die Linienform des ersten Phononenwiederholers ist gegeben durch [Kli75]

$$
\begin{aligned}
S(\hbar \omega) & =A \cdot X^{\frac{3}{2}} \cdot \exp \left(\frac{-X}{k_{b} T}\right) \\
X & =\hbar \omega+\hbar \omega_{\mathrm{LO}}-E_{G}(T)+E_{E x}^{b} .
\end{aligned}
$$

Die Exzitonbindungsenergie $E_{E x}^{b}$ wurde auf $60 \mathrm{meV}$, die LO-Phononenenergie auf 72 meV gesetzt. Die Amplitude, die Temperatur und die temperaturabhängige 
Bandlücke $E_{G}(T)$ werden numerisch angepasst. Die Anpassung der Linienform ist in Abbildung 3.11 b) als blaue Linie aufgetragen. Aus der Anpassung ergibt sich eine Bandlücke von 3,435 eV, was eine Blauverschiebung um 19 meV bedeutet (vgl. Abschnitt 3.2). Dieses Ergebnis ist als untere Abschätzung des quantum confinements zu verstehen, da die Exzitonbindungsenergie in niedrigdimensionalen Systemen deutlich zunehmen kann [Sen03, Sun01]. Eine ähnliche Vergrößerung der Bandlücke wurde an Zinkoxid-Nanopartikeln vergleichbaren Durchmessers in Absorptionsmessungen [Mäd02] und Photolumineszenzmessungen [Lin06] beobachtet sowie durch theoretische Berechnungen mit einer tight-binding-Methode vorhergesagt [Vis03]. Als weiterer Parameter wurde die Temperatur mit Gleichung $3.5 \mathrm{zu}$ $74 \mathrm{~K}$ angepasst. Dieser Wert weicht stark von der auf dem Probenhalter gemessen Temperatur ab, was auf die relativ schlechte thermische Ankopplung der Drähte zurückzuführen ist. Weiterhin wird die Größenverteilung der Drähte zu einer Verbreiterung der Linien im Spektrum des Nanodrahtensembles führen, was auch der Grund für die Abweichung zwischen den Messdaten und der Anpassung an der niederenergetischen Flanke sein kann.

Um weiteren Aufschluss über die Zusammensetzung der Lumineszenz zu bekommen, ist die Untersuchung an ausschließlich ultradünnen Nanodrähten notwendig. Dazu müssen die ultradünnen Drähte von den dickeren getrennt werden. Dies wurde versucht, indem die Nanodrähte mit geringer Dichte auf ein zuvor im FIB markiertes TEM-Netzchen mit geringer Dichte übertragen wurden. Im TEM wurde die Position verschiedener Nanodrähte relativ zu den Markierungen kartiert. Die Lumineszenzuntersuchungen erfolgten in einem Mikrophotolumineszenzaufbau in der Kollaboration Voss/Bremen, wo die Positionen der Drähte entsprechend angefahren werden konnten. Zur Anregung diente hier ein frequenzverdoppelter Ti:SaphirLaser. Das Spektrum eines ultradünnen Drahtes bei einer nominellen Temperatur von $7 \mathrm{~K}$ ist in Abbildung $3.11 \mathrm{c}$ ) gezeigt. Das Spektrum ist vergleichbar mit den Messungen an dem Nanodrahtensemble (vgl. Abbildung 3.10 b)). Eine spektrale Verschiebung des Spektrums in Abbildung $3.11 \mathrm{c}$ ) um $\approx 4 \mathrm{meV}$ zu niedriger Energie ist auf eine laserinduzierte lokale Erwärmung der Drähte zurückzuführen, die sich aufgrund der unterschiedlichen Anregung von den Messungen am Ensemble unterscheidet. Diese Messungen konnten bestätigen, dass auch bei den ultradünnen Drähten die Lumineszenzeigenschaften des Ensembles die der einzelnen Drähte gut 
wiedergeben.

\subsection{Hochanregungsspektroskopie}

Wie die Untersuchungen in den oberen Abschnitten gezeigt haben, sind die optischen Eigenschaften von Zinkoxid bei moderaten Anregungsdichten durch gebundene und freie Exzitonen geprägt. Da der Abstand der Exzitonen groß gegenüber dem Exzitonbohrradius ist sind die Eigenschaften der Lumineszenz linear von der Anregungsdichte abhängig. Wird nun die Anregungsdichte sukzessiv erhöht, so wird auch die Dichte der Exzitonen größer, sodass diese in Wechselwirkung treten können. Dies hat nichtlineare optische Effekte zur Folge.

Abbildung 3.12 a) zeigt eine Reihe von Lumineszenzspektren eines ZinkoxidNanodrahtensembles, die unter steigender Anregungsdichte aufgenommen wurden. Die Spektren sind zur besseren Übersicht entlang der Ordinate verschoben aufgetragen und normiert. Alle Spektren wurden bei einer Probentemperatur von $16 \mathrm{~K}$ mit einem CCD-Detektor aufgenommen. Das unterste Spektrum zeigt die Lumineszenz unter moderater Anregung durch einen HeCd-Laser (cw). Die Anregungsdichte beträgt $\approx 0,5 \mathrm{Wcm}^{-2}$. Das Spektrum ist durch die Lumineszenz gebundener Exzitonen (DX) dominiert. Die Position des freien Exzitons (FX) ist mit einer gestrichelten Linie markiert. Die übrigen Spektren wurden unter Anregung durch einen gepulsten, frequenzvervierfachten Nd:YAG-Laser aufgenommen. Das Spektrum, das mit der geringsten Anregungsdichte aufgenommen wurde zeigt bereits eine deutlich andere Charakteristik als bei cw-Anregung. An der niederenergetischen Flanke des DX-Peaks ist bei 3,357 eV eine weitere Lumineszenzbande zu erkennen, deren Breite (FWHM) etwa 5 meV beträgt. Bei steigender Anregungsdichte wird diese Linie deutlich intensiver, breiter und verschiebt um etwa $5 \mathrm{meV}$ zu niedrigeren Energien. Diese Linie ist entsprechend der Literatur als M-Bande bezeichnet und taucht typischerweise $\approx 25 \mathrm{meV}$ unterhalb des freien Exzitons auf. Als Ursache für diese Bande wird in der Literatur meist die strahlende Rekombination von Biexzitonen genannt [Hva83]. Die beobachtete Biexzitonbindungsenergie beträgt in Zinkoxid etwa 12 - $20 \mathrm{meV}$ [Yam01]. Auch die leichte Verschiebung der M-Bande mit steigender Anregungsdichte wird in der Literatur beschrieben [But98].

Ab einer Anregungsdichte von 0,2 $\mathrm{MW} \mathrm{cm}^{-2}$ taucht bei etwa $3,328 \mathrm{eV}$ eine weitere 

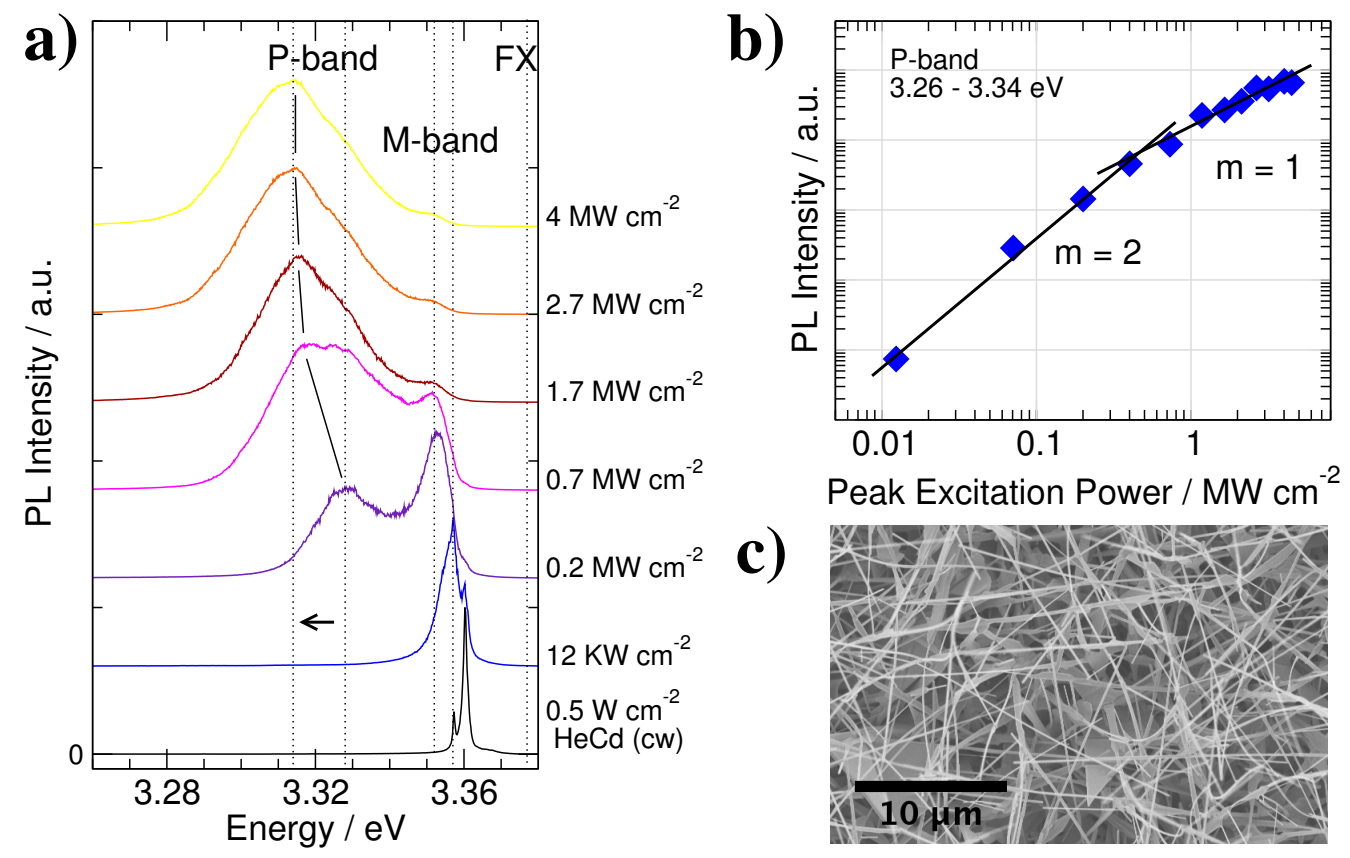

Abbildung 3.12: Lumineszenz eines Zinkoxid-Nanodrahtensembles unter Hochanregung. a) Bei steigender Anregungsdichte sind zwei neue Linie im Spektrum zu erkennen, die mit M- und P-Band gekennzeichnet sind. b) Die Intensität der P-Bande als Funktion der Anregungsdichte $I_{\text {exc }}$. Die PL-Intensität steigt zunächst superlinear $\propto I_{e x c}^{2}$. Ab einem Schwellwert von etwa $0,3 \mathrm{MW} \mathrm{cm}{ }^{-2}$ steigt die PL-Intensität dann $\propto I_{e x c}$ an. c) SEM-Aufnahme des untersuchten Nanodrahtensembles. Unter den Nanodrähten sind mikrokristalline, bandartige Strukturen zu erkennen.

Lumineszenzbande auf, die als P-Bande bezeichnet wird. Bei der P-Bande ist mit steigender Anregungsdichte eine Rotverschiebung um etwa $14 \mathrm{meV}$ zu beobachten. Bei hohen Anregungsdichten dominiert die P-Bande das Spektrum. Als Ursprung der P-Bande wird in der Literatur die inelastische Exziton-Exziton-Streuung genannt. Bei der inelastischen Streuung zweier Exzitonen im Grundzustand $n=1$ wird das eine Exziton in einen höheren Zustand $(\mathrm{n}=2,3, . ., \infty)$ angeregt, während das zweite unter der Emission von Licht zerfällt. Die Energie des emittierten Lichtes ist dabei gegeben durch [Kli75]

$$
E_{P}(n)=E_{g a p}(T)-E_{b}^{E x}\left(2-\frac{1}{n^{2}}\right)-\frac{3}{2} k_{B} T .
$$

Dabei bezeichnet $E_{\text {gap }}(T)$ die Bandlücke (vgl. Gleichung 3.2) und $E_{b}^{E x} \approx 60 \mathrm{meV}$ die Exzitonbindungsenergie. Eine Berechnung für $n=2$ führt zu einem Wert von 
3,330 eV bei $16 \mathrm{~K}$, was in guter Übereinstimmung mit der Position der P-Bande bei einer Anregungsdichte von 0,2 $\mathrm{MW} \mathrm{cm}^{-2}$ ist. Eine Erhöhung der Anregungsdichte führt entsprechend zu einer größeren Wahrscheinlichkeit in höhere Zustände $\mathrm{zu}$ streuen, was eine spektrale Rotverschiebung von maximal $\frac{1}{4} \cdot E_{b}^{E x}=15 \mathrm{meV}$ für $\mathrm{n}=\infty$ bedeutet. Die beobachtete Verschiebung bei der höchsten Anregungsdichte beträgt $14 \mathrm{meV}$ und ist damit ebenfalls in guter Übereinstimmung mit Gleichung 3.5. Wird die Anzahl der Exzitonen im Anregungsvolumen erhöht, so steigt die Wahrscheinlichkeit für einen Streuprozess quadratisch dazu an [Wün80, Pri04]. Folglich sollte auch die Intensität der P-Bande eine quadratische Funktion der Anregungsintensität sein. In Abbildung 3.12 b) ist die integrale Intensität der PL im Bereich der P-Bande (3,26 - 3,34 eV) als Funktion der Anregungsdichte doppelt logarithmisch aufgetragen. Bis zu einer Anregungsdichte von $\approx 1 \mathrm{MW} \mathrm{cm}^{-2}$ liegen die gemessenen Werte auf einer Geraden mit der Steigung 2; folglich ist die Intensität der Lumineszenz in diesem Bereich eine quadratische Funktion der Anregungsdichte. Bei höheren Anregungsdichten wächst die Intensität weiter linear an, was vermutlich auf eine Sättigung zurückzuführen ist.

Auch wenn die Intensität der P-Bande stärker zunimmt als die Anregungsintensität, ist dieser Emissionsprozess als spontane Emission zu verstehen [Wün80]. Allerdings bietet dieser Rekombinationskanal durchaus Potential für stimulierte Emission. Eine genauere Betrachtung der inelastischen Exzitonstreuung zeigt, dass sich dieser Prozess energetisch als Vierniveausystem auffassen lässt. Ausgangspunkt sind zwei Exzitonen im Grundzustand (1). Nach dem Streuprozess befindet sich ein Exziton in einem energetisch niedrigerem Zustand (2) und rekombiniert unter Aussendung von Licht (3). Das zweite Exziton befindet sich nach dem Streuprozess in einem angeregtem Zustand (4) aus dem es direkt wieder in den Grundzustand (1) übergeht. Damit ist eine Besetzungsinversion gegeben, sobald zwei Exzitonen im Grundzustand und die angeregten exzitonischen Zustände unbesetzt sind [Kli07]. Dieses Verhalten, dass sich durch eine Verschmälerung der P-Bande und ein mehr als kubisches Wachstum der PL-Intensität abzeichnet, wurde an dünnen Zinkoxidschichten beobachtet [Pri04].

Ein vergleichsweise intensives und dominantes Auftreten der P-Bande, wie es Abbildung 3.12 a) zeigt, wird in der Literatur fast ausschließlich von Zinkoxidschichten und Einkristallen hoher Qualität berichtet. Entsprechend stellt sich die Frage, wie 

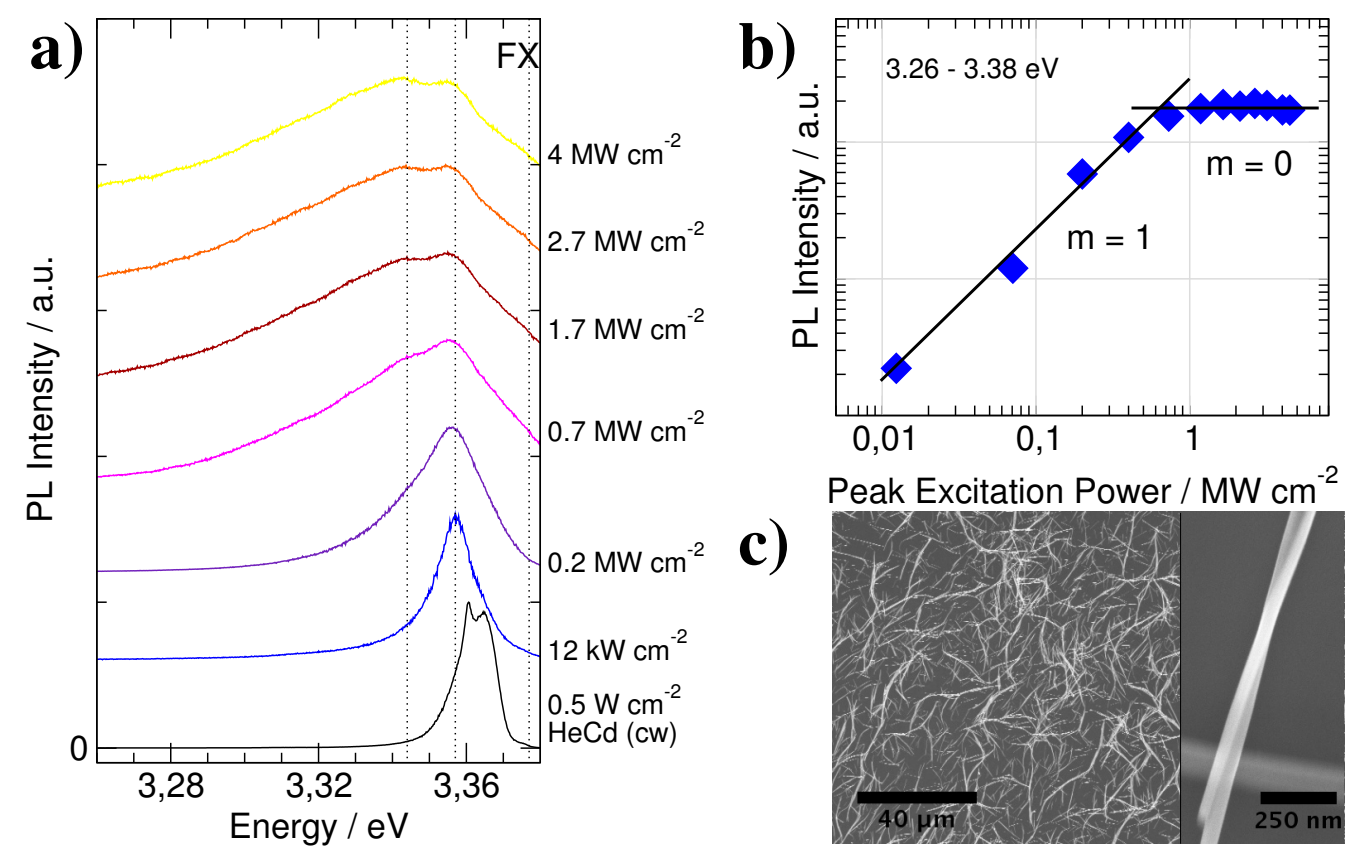

Abbildung 3.13: Lumineszenz dünner Zinkoxid-Nanodrähte unter Hochanregung. a) Bei steigender Anregungsintensität verbreitert die Lumineszenzbande leicht. Es sind im Wesentlichen zwei Schultern erkennbar. b) Die Intensität der Lumineszenz als Funktion der Anregungsintensität $I_{\text {exc }}$. Die PL-Intensität steigt zunächst linear an und geht dann bei etwa $0,7 \mathrm{MW} \mathrm{cm}^{-2}$ in Sättigung. c) SEM-Aufnahme des untersuchten Nanodrahtensembles.

sich die Hochanregungsspektren verändern, wenn die Strukturgröße verringert wird. Untersucht wurden im Folgenden Zinkoxid-Nanodrähte mit einem mittleren Durchmesser von $\approx 50 \mathrm{~nm}$, die nach der Synthese über eine Suspension auf ein sauberes Substrat übertragen wurden (vgl. Abbildung 3.13 c)). Die Nanodrähte wurden in der Gruppe Li/Hong Kong hergestellt. Die leistungsabhängigen Lumineszenzspektren der Probe in Abbildung 3.13 a) zeigen ein deutlich anderes Verhalten als in Abbildung 3.12 a). Bei niedrigen Anregungsdichten ist die Lumineszenz dieser Probe ebenfalls durch donatorgebundene Exzitonen geprägt. Bei mittleren Anregungsdichten dominiert eine Bande bei 3,357 eV was im Bereich der M-Bande liegen würde. Bei hohen Anregungsdichten gibt es $\mathrm{ab} \approx 0,7 \mathrm{MW}^{-2}$ eine weitere Schulter bei 3,344 eV. Beide Banden zeigen keine spektrale Verschiebung mit zunehmender Leistung. Auch der Intensitätsverlauf der Lumineszenz in Abbildung 3.13 b) unterscheidet sich deutlich von dem in Abbildung 3.13 b). Die Lumineszenz der 
dünnen Nanodrähte nimmt zunächst nahezu linear mit der Anregungsdichte zu. $\mathrm{Ab}$ einer Anregungsdichte von etwa $\approx 0,7 \mathrm{MW} \mathrm{cm}^{-2}$ bleibt die Lumineszenz dann unverändert. Die Ursache für das Ausbleiben der P-Bandenlumineszenz in dünnen Nanodrähten fand Hauschild in den photonischen Eigenschaften der Nanodrähte begründet [Hau06a]. Er konnte rechnerisch zeigen, dass Zinkoxid-Nanodrähte unterhalb von $130 \mathrm{~nm}$ im Energiebereich der P-Bande (3,32 eV) single mode-Wellenleiter sind [Hau06b]. Die geführten Moden lassen sich dann einer von der Drahtlänge abhängigen, diskreten Energie zuordnen. Ein durch inelastische Exziton-ExzitonStreuung erzeugtes Photon kann nur dann emittiert werden, wenn seine Energie mit der Energie einer geführten Mode übereinstimmt. Die Anzahl der geführten Moden nimmt mit dem Drahtdurchmesser quadratisch ab. Damit ist die P-Bande für dünne Nanodrähte effektiv unterdrückt.

\subsection{Donator-Akzeptor-Paarübergang in Zinkoxid}

Wie bereits in der Einleitung erläutert, fehlt es an einer Methode zur effektiven und reproduzierbaren p-Dotierung von Zinkoxid [Fon06]. Dies gilt für Nanodrähte gleichermaßen wie für Volumenmaterial. Üblicherweise zeigen nominell intrinsisch hergestellte Zinkoxid-Kristalle n-typ Verhalten mit einer Donatorhintergrundkonzentration im Bereich von $10^{16} \mathrm{~cm}^{-3}$. Diese muss überwunden werden, bevor eine effektive p-typ Leitung einsetzen kann. In der Literatur sind einige Berichte über erfolgreiche p-Dotierung zu finden [Lu07, Loo04]. Es hat sich sowohl durch theoretische Berechnungen als auch durch die erfolgreiche Verwendung in anderen IIVI-Halbleitern herausgestellt, dass Stickstoff der vielversprechendste Akzeptor in Zinkoxid ist [Fon06]. Insbesondere weil Sauerstoff und Stickstoff ähnliche ionische Radien haben, ist Stickstoff potentiell der flachste Gruppe-V-Akzeptor in Zinkoxid. Gruppe-I-Elemente sind weniger geeignete Akzeptoren, da sie, abhängig davon, ob sie auf einem Zink- oder Sauerstoffplatz eingebaut werden, ein amphoteres Verhalten zeigen [Loo04, Kli07, Par02].

In diesen Untersuchungen wurde Stickstoff durch Ionenimplantation in ein ZinkoxidNanodrahtensemble implantiert, wie es in Abbildung 3.2 a) dargestellt ist. Die Implantation wurde an dem Beschleuniger Ionas (vgl. Abschnitt 2.2.1) mit acht unterschiedlichen Ionenenergien durchgeführt. So konnte eine homogene Stick- 
a)

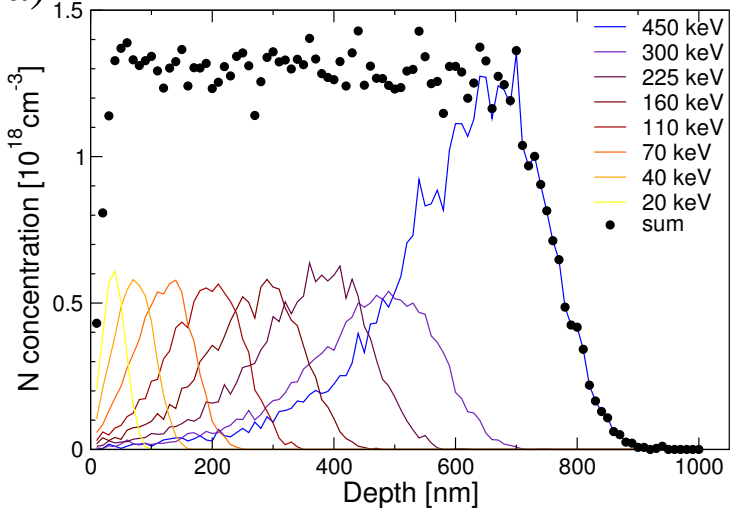

b)

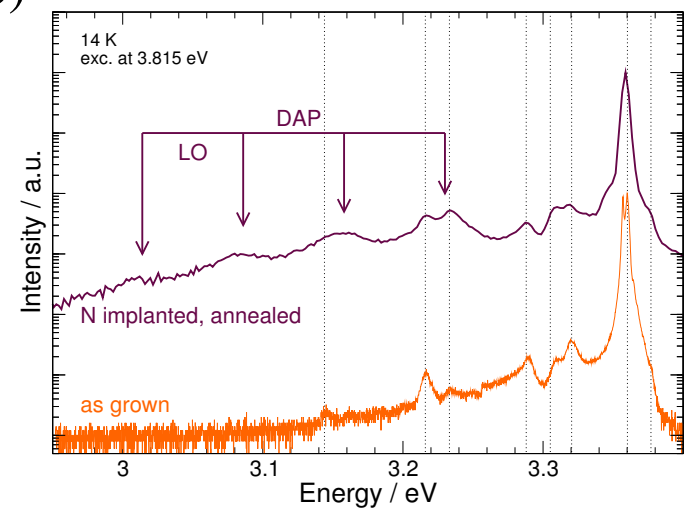

Abbildung 3.14: a) Implantationsprofil für Stickstoff in Zinkoxid, wie es mit der Software TRIM berechnet wurde. Eine Überlagerung von acht Ionenenergien resultiert bei der gewählten Fluenz in einer homogenen Stickstoffkonzentration bis zu einer Tiefe von $700 \mathrm{~nm}$. b) Ein Vergleich der Lumineszenz der Probe vor und nach der Implantation. Bei einer Energie von 3,230 eV tritt eine breite Linie auf (DAP), der zu niedrigen Energien Phononenwiederholer folgen.

stoffkonzentration in den teils überlagerten Nanodrähten erreicht werden. Abbildung 3.14 a) zeigt das Stickstoffkonzentrationsprofil, wie es mit dem Monte-Carlo Code TRIM [Zie08] berechnet wurde. Die Stickstoffkonzentration beträgt entsprechend der Simulation $\approx 1,3 \cdot 10^{18} \mathrm{~cm}^{-3}$ über eine effektive Tiefe von $700 \mathrm{~nm}$. So ist gewährleistet, dass nahezu das gesamte in der PL angeregte Volumen homogen implantiert wurde (vgl. Abbildung 3.2 b)). Um die Kristalldefekte auszuheilen, die während der Implantation entstehen, wurde die Probe bei $600{ }^{\circ} \mathrm{C}$ für $30 \mathrm{~min}$ in Vakuum $\left(1 \cdot 10^{-6} \mathrm{mbar}\right)$ getempert.

Abbildung 3.14 b) zeigt die Lumineszenzspektren der Probe vor und nach der Implantation. Die Spektren wurden bei einer Temperatur von $14 \mathrm{~K}$ aufgenommen und sind normiert, sowie entlang der Ordinate verschoben aufgetragen. Das Spektrum der unimplantierten Nanodrähte zeigt die typischen Banden des freien und gebundener Exzitonen sowie deren Phononenwiederholer (vgl. Abschnitt 3.1). Das Spektrum der implantierten und getemperten Probe wird ebenfalls von der Lumineszenz gebundener Exzitonen dominiert. Die Halbwertsbreite der Bande ist leicht verbreitert, was darauf hinweist, dass die Kristalldefekte noch nicht vollkommen ausgeheilt sind. Die Bande des freien Exzitons ist zudem etwas stärker 
ausgeprägt als dessen erster Phononenwiederholer. Bei einer Energie von 3,230 eV taucht im Spektrum der implantierten Drähte mit sehr geringer Intensität eine Bande auf, die bei der nicht implantierten Probe nicht zu beobachten ist. Zu niedriger Energie folgen auf diese Bande mit LO-Abstand Phononenwiederholer. Da dies charakteristisch für Donator-Akzeptor-Paarübergänge ist und auch in der Literatur eine solche Zuordnung durchgeführt wurde, wird diese Bande im Folgenden als DAP bezeichnet. Ein Vergleich mit Untersuchungen an Zinkoxid-Kristallen, die während des Wachstums bzw. anschließend z.B. durch Ionenimplantation mit Stickstoff dotiert wurden [Sti08a, Rom03, Tsu05, Mey05, Xio05], zeigt, dass in den meisten dieser Veröffentlichungen [Mey05, Xio05, Zeu02, Loo02] eine Bande bei 3,235 eV beobachtet wurde. Diese Bande wird meist einem DAP zugeordnet, allerdings wird auch ein Phononenwiederholer eines neutralen akzeptorgebundenen Exzitons $\left(\mathrm{A}^{0} \mathrm{X}\right)$ [Loo02] sowie der Übergang eines freien Elektrons zu einem Akzeptorniveau (e,A) [Rom03, Tho01] als mögliche Ursache genannt. Zusätzlich wird in einigen Veröffentlichungen auch eine Bande bei 3,315 eV(3,309 eV) [Rom03, Loo02] beobachtet, die ebenfalls einem $\mathrm{A}^{0} \mathrm{X}$ zugeordnet wird. Da der Ursprung der DAPBande noch kontrovers diskutiert wird und neben den vorgeschlagenen Identifizierungen diese Bande spektral nahezu an der selben Position auftritt wie die zweiten Phononenwiederholer des freien und der donatorgebundenen Exzitonen, soll diese Bande näher untersucht werden. Um diese Untersuchungen durchzuführen sind allerdings größere Intensitäten der Lumineszenz notwendig. Dies konnte auch durch eine Veränderung der Stickstoffkonzentration und durch Variieren der Temperbedingungen nicht erreicht werden. Entsprechend wurde versucht, eine höhere Intensität der Bande an kommerziell erhältlichen ${ }^{1}$, hydrothermal gezüchteten ZinkoxidEinkristallen zu erzielen [Sti08a]. An diesen Kristallen wurden die folgenden Untersuchungen durchgeführt.

Die Kristalle wurden mit dem gleichen Implantationsprofil, wie es in Abbildung 3.14 a) dargestellt ist, implantiert. Anschließend wurde der Ausheilprozess untersucht. Dazu wurde die Probe bei unterschiedlichen Temperaturen $\left(300{ }^{\circ} \mathrm{C}\right.$ $600{ }^{\circ} \mathrm{C}$ ) für $30 \mathrm{~min}$ im Vakuum $\left(1 \cdot 10^{-6}\right.$ mbar) getempert, wobei nach jedem Temperaturschritt Photolumineszenzuntersuchungen durchgeführt wurden. Abbildung 3.15 zeigt die normierten Lumineszenzspektren zusammen mit dem Spektrum

\footnotetext{
${ }^{1}$ CrysTec GmbH, Berlin
} 

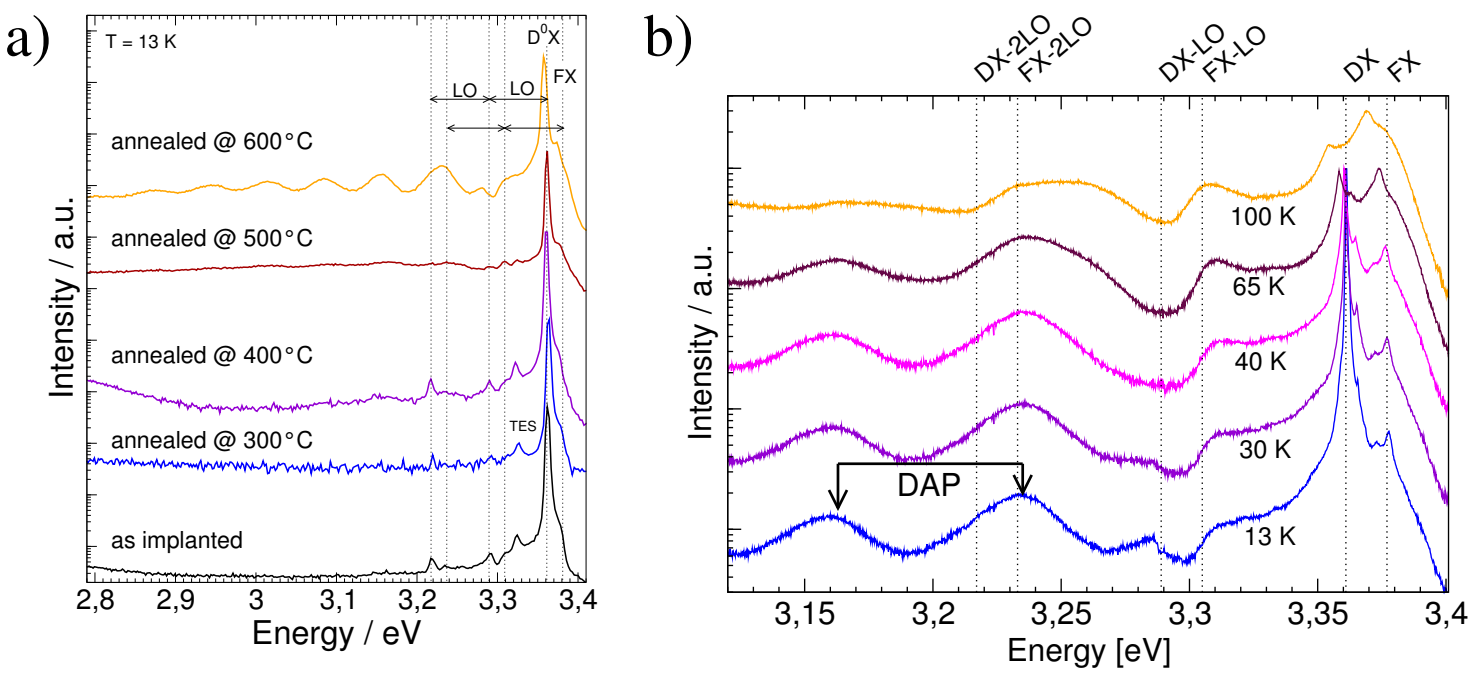

Abbildung 3.15: a) Lumineszenz von Stickstoff implantierten Zinkoxid-Einkristallen bei unterschiedlichen Ausheilbedingungen. Ab einer Temperatur von $500{ }^{\circ} \mathrm{C}$ tritt eine Bande bei $3,230 \mathrm{eV}$ auf, die nach weiterem Tempern bei $600^{\circ} \mathrm{C}$ bis zu fünf Phononenwiederholer zeigt. b) Temperaturabhägigkeit der Lumineszenz eines Stickstoffimplantierten Zinkoxid-Kristalls, der bei $600{ }^{\circ} \mathrm{C}$ getempert wurde. Die Anregung erfolgte moderat bei 3,815 eV.

der Probe direkt nach der Implantation auf einer logarithmischen Skala und entlang der Ordinate verschoben. Die Lumineszenz wurde bei moderater Anregung $(3,815 \mathrm{eV})$ bei einer Temperatur von $13 \mathrm{~K}$ aufgenommen. Das Spektrum der Probe wird direkt nach der Implantation noch immer durch die Lumineszenz gebundener Exzitonen dominiert, was darauf hinweist, dass das Material eine große Strahlenhärte hat [Nag75, Kuc03]. Im Detail zeigt das Spektrum, ähnlich wie nicht implantierte Nanodrähte, die FX- und $\mathrm{D}^{0} \mathrm{X}$-Lumineszenz sowie deren Phononenwiederholer. Die Positionen der einzelnen Banden sind durch gestrichelte Linien in Abbildung 3.15 gekennzeichnet. Nach den ersten beiden Temperschritten bei $300{ }^{\circ} \mathrm{C}$ und $400{ }^{\circ} \mathrm{C}$ lassen sich keine signifikanten Änderungen im Spektrum erkennen. Eine weiter Temperaturbehandlung bei $500{ }^{\circ} \mathrm{C}$ führt zu einer leichten Erhöhung des Hintergrunds zwischen 2,9 eV und 3,2 eV. In diesem Spektralbereich kommt nach einem weiteren Temperschritt bei $600{ }^{\circ} \mathrm{C}$ eine periodische Bande auf, deren erste Linie bei 3,230 eV liegt. Auf diese Linie folgen zu niedriger Energie mit Abständen von $72 \mathrm{meV}$ fünf weitere Linien mit geringerer Intensität, ähnlich wie es im Spektrum der implantierten Nanodrähte beobachtet wurde. Die Intensität der Linie ist in der 
Tat deutlich höher, wodurch weitere Untersuchungen an der Bande möglich sind. Zunächst soll ausgeschlossen werden, dass es sich bei der DAP-Bande um die zweiten Phononenwiederholer des freien oder gebundener Exzitonen handelt, die spektral an der selben Position erwartet werden. Ein erster Hinweis darauf ist, dass die Intensität der Bande höher ist als die der ersten Phononenwiederholer von dem freien und der gebundenen Exzitonen. Allerdings wurde in der Literatur gezeigt, dass die zweiten Phononenwiederholer durchaus höhere Intensität im Lumineszenzspektrum zeigen können als die ersten [Per82, Luc02]. Im Folgenden werden temperaturabhängige PL-Messungen vorgestellt, aufgrund derer auszuschließen ist, dass es sich bei der DAP-Bande um Phononenwiederholer handelt. Abbildung 3.15 b) zeigt eine Reihe von Lumineszenzspektren, die bei Probentemperaturen zwischen $13 \mathrm{~K}$ und $100 \mathrm{~K}$ aufgenommen wurden. Die Spektren werden von der Lumineszenz des freien und gebundener Exzitonen dominiert, wobei die relative Intensität der freien Exzitonen mit höheren Temperaturen zunimmt. Dies wurde in Abschnitt 3.2 auf eine zunehmende thermische Dissoziierung der donatorgebundenen Exzitonen zurückgeführt. Ensprechend wird erwartet, dass auch die Intensität der Phononenwiederholer der gebundenen Exzitonen geringer wird. Dieser Effekt wird auch beobachtet: bereits bei $40 \mathrm{~K}$ ist der erste Phononenwiederholer der gebundenen Exzitonen nicht mehr im Spektrum zu erkennen. Dabei wird keine Veränderung der DAP-Bande beobachtet und so ausgeschlossen, dass der zweite Phononenwiederholer großen Beitrag zu der Bande hat. Gleichzeitig nimmt die Intensität der FXLumineszenz und somit auch derer Phononenwiederholer zu. Ein Wachstum der Intensität kann am ersten Phononewiederholer deutlich beobachtet werden, während sich an der hochenergetischen Flanke der DAP-Bande eine Schulter ausbildet $(65 \mathrm{~K})$, die dem zweiten Phononenwiederholer zuzuordnen ist. Dies bedeutet, dass der zweite Phonenwiederholer spektral etwas höherenergetisch als die DAP-Bande auftritt. Somit konnte gezeigt werden, dass es sich bei der DAP-Bande weder um den Phononenwiederholer des freien, noch der gebundenen Exzitonen handelt.

Um die Identifikation der DAP-Bande weiter zu bestärken, wurden die folgenden zeitabhängigen und leistungsabhängigen Lumineszenzmessungen an dieser Bande durchgeführt, die eine eindeutige Identifikation als Donator-AkzeptorPaarübergang zulassen. Die Energie der Photonen, die durch einen DonatorAkzeptor-Paarübergang erzeugt werden, ist durch die Differenz von Akzeptor- und 
a)

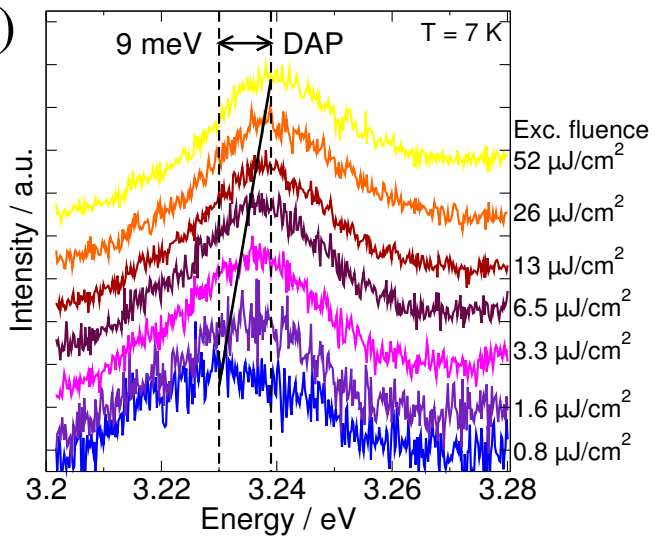

b)

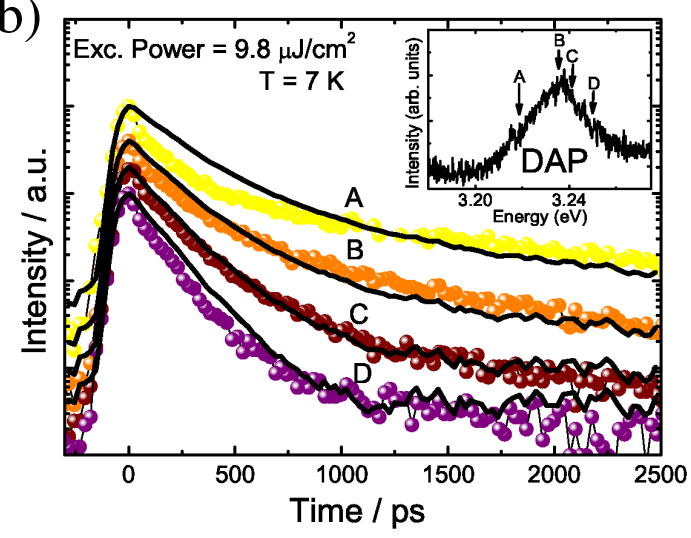

Abbildung 3.16: a) Leistungsabhängigkeit der DAP-Bande in stickstoffimplanierten ZinkoxidKristallen. Mit steigender Anregungsdichte verschiebt die DAP-Bande zu höheren Energien b) Transienten an verschiedenen spektralen Positionen in der DAP-Bande. Die Transienten zeigen einen multiexponentiellen Zerfall.

Donatorniveau und durch die Coulombenergie der ionisierten Zuständen bestimmt [Bäu00, Kli05]:

$$
E_{D A P}=E_{g a p}-E_{D}-E_{A}+\frac{e^{2}}{4 \pi \epsilon_{0} \epsilon(0) r_{D A}},
$$

dabei bezeichnen $E_{D}$ und $E_{A}$ die Donator- und Akzeptorbindungsenergie, $E_{\text {gap }}$ die Bandlücke, $\epsilon(0)$ die Dielektrizitätskonstante und $r_{D A}$ den Abstand zwischen Donator und Akzeptor. Nach dem Standardmodell für Donator-Akzeptor-Paarübergänge [Tho65] werden Donator-Akzeptor-Paare mit großem Abstand bei geringen Anregungsdichten zuerst besetzt. Mit zunehmender Anregungsdichte nimmt die Anzahl der freien Ladungsträger zu, was dazu führt, dass auch Donator-Akzeptor-Paare mit geringerem Abstand besetzt werden. Entsprechend wird eine Blauverschiebung der DAP-Bande mit zunehmender Anregungsdichte erwartet.

Abbildung 3.16 a) zeigt Spektren der DAP-Bande bei zunehmender Anregungsdichte, wie sie in der Bremer Kollaboration gemessen werden konnten. Die Spektren wurden bei einer Temperatur von $7 \mathrm{~K}$ unter Anregung mit einem Titan:SaphirLaser gemessen. Mit steigender Anregungsdichte $\left(0,8 \mu \mathrm{J} / \mathrm{cm}^{2}-52 \mu \mathrm{J} / \mathrm{cm}^{2}\right)$ wird in Übereinstimmung mit dem Modell eine spektrale Verschiebung des Maximums der DAP-Bande um 9 meV beobachtet, was die Identifizierung als DAP-Bande untermauert. 
Eine weitere Möglichkeit die DAP als solche zu identifizieren besteht darin, die Dynamik der Lumineszenz an verschiedenen Positionen in der DAP-Bande zu untersuchen [Tho65]. Entsprechend wurden in der Bremer Kollaboration Transienten an vier unterschiedlichen Stellen in der DAP gemessen. Die Transienten wurden mit der Methode des zeitkorellierten Einzelphotonennachweis gemessen [Wis07]. Die gemessenen Transienten sind in Abbildung 3.16 b) normiert und zur besseren Übersicht entlang der Ordinate verschoben aufgetragen. Im Einsatz der Abbildung ist das zeitintegrierte Spektrum der DAP-Bande gezeigt. Hier sind die vier Positionen markiert, an denen die Transienten gemessen wurden (A: 3,220 eV, B: 3,238 eV, C: 3,241 eV, D: 3,255 eV). Die Transienten können nun mit dem Modell von Thomas et. al. beschrieben werden [Tho65]. Es wird zunächst angenommen, dass Donatoren Majoritätsstörstellen im Kristall sind. Aufgrund ihrer statistischen Verteilung haben dann insbesondere die Akzeptoren verschiedene lokale Umgebungen, was zu verschiedenen Lebenszeiten führt. Der zeitliche Verlauf der Intensität der Lumineszenz an einer energetischen Position E in der DAP ist dann gegeben durch [Tho65]:

$$
I_{E}(t)=W\left(r_{D A}\right) \cdot \exp \left(-W\left(r_{D A}\right) t\right) \exp \left[4 \pi N_{D} \int_{0}^{\infty}(\exp (-W(r) t)-1) r^{2} d r\right]
$$

Die Übergangsrate $W\left(r_{D A}\right)=W_{0} \exp \left(-2 r_{D A} / a_{D}\right)$ wird durch den Abstand zwischen Akzeptor und Donator sowie den Bohrradius des Donators bestimmt. Folglich wird ein multiexponentieller Zerfall erwartet der bei höheren Energien schneller wird, da bei kleinerem Doantor-Akzeptor-Abstand $r_{D A}$ die Überlappung zwischen den Wellenfunktionen von Elektron und Loch größer wird (vgl. Gleichung 3.6). Alle Transienten zeigen das erwartete multiexponentielle Verhalten sowie die erwartete Abhängigkeit von der spektralen Position. Es werden mehrere unabhängige Konstanten benötigt, um die Messwerte mit Gleichung 3.7 anzupassen. Da die Akzeptorkonzentration sehr klein und die Bindungsenergie der beteiligten Donatoren und Akzeptoren nicht bestimmt werden kann, wird eine Akzeptorbindungsenergie von $E_{A}=165 \mathrm{meV}$ und eine Donatorbindungsenergie von $E_{D}=53 \mathrm{meV}$ aus der Literatur übernommen [Zeu02]. Die Bandlücke wird zu 3,345 eV [Mey04], die Dielektrizitätskonstante zu $\epsilon(0)=8$ angenommen [LaB99]. Der Abstand von 


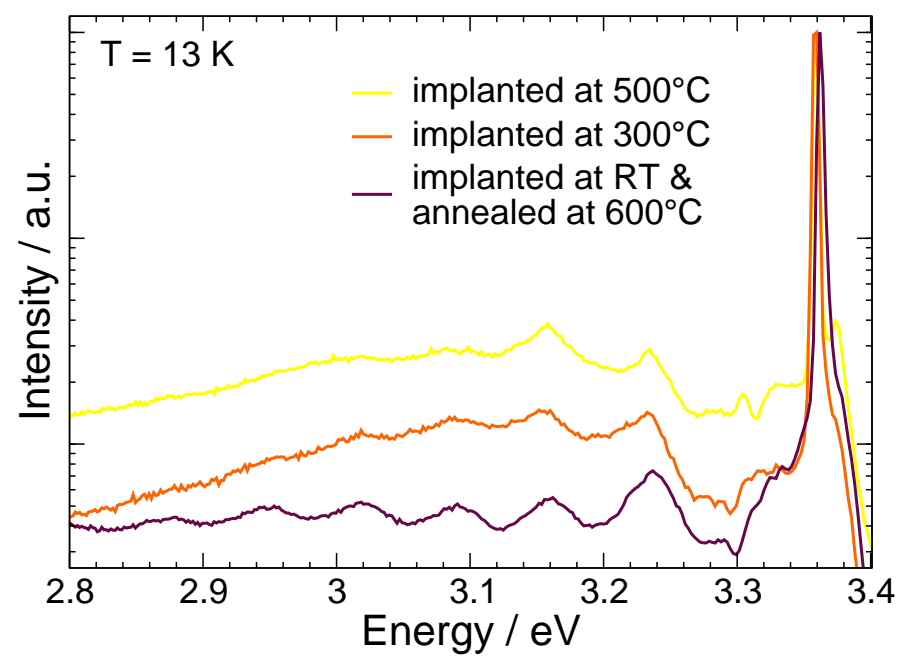

Abbildung 3.17: Lumineszenzspektren von Zinkoxid-Kristallen, die bei hohen Temperaturen mit $\mathrm{N}$ implantiert wurden. Im Vergleich dazu ist das Spektrum eines Kristalls gezeigt, der bei Raumtemperatur implantiert und anschließend bei $600{ }^{\circ} \mathrm{C}$ getempert wurde.

Donator und Akzeptor $r_{D A}$ wurde numerisch mit Gleichung 3.6 berechnet. Die durchgezogenen Linien in Abbildung 3.16 zeigen die mit Gleichung 3.7 berechneten Transienten. Dabei wurde eine Donatordichte von $\mathrm{N}_{\mathrm{D}}=(4,7 \pm 0,3) \cdot 10^{17} \mathrm{~cm}^{-3}$, ein Donator Bohrradius von $\mathrm{a}_{\mathrm{D}}=(2,0 \pm 0,1) \mathrm{nm}$ und eine Übergangsrate von $\mathrm{W}_{0}=(2,9 \pm 0,1) \cdot 10^{11} \mathrm{~s}^{-1}$ eingesetzt. Diese Werte sind in guter Übereinstimmung mit Ref. [Tsu05]. Bei den spektralen Positionen B-D liegen die berechneten und gemessenen Werte eng beieinander, was die Identifizierung als Donator-AkzeptorPaarübergang zusätzlich bestätigt. Die Abweichung an Position A bei Zeiten bis 750 ps lässt sich durch den Überlapp mit den zweiten Phononenwiederholern erklären (vgl. Abbildung 3.15 b)). Durch deren spektralen Überlapp sind auch die Transienten überlagert, was schließlich zu der beobachteten Abweichung führt.

Es konnte also gezeigt werden, dass eine Bande, die nach dem Tempern von stickstoffimplanierten Zinkoxid-Kristallen in deren Lumineszenzspektrum bei $\approx 3,230 \mathrm{eV}$ auftritt, alle Eigenschaften eines Donator-Akzeptor-Paarübergangs erfüllt. Lediglich die Intensität der Bande ist noch sehr gering, was darauf hinweist, dass die Effektivität, d.h. der Prozentsatz optisch aktivierter Akzeptoren, bei diesem Dotierprozess sehr gering ist. Um diese zu erhöhen ist eine Verbesserung des Temperprozesses entscheidend. Ein erster Schritt wäre es, die Temperatur weiter zu erhöhen. Allerdings 
würde dies, wie bei den meisten Verbindungshalbleitern, zu einer Zersetzung führen. Der flüchtigere Bestandteil der Verbindung, in diesem Fall Sauerstoff, würde zuerst verdampfen, wodurch der Kristall nicht mehr stochiometrisch bliebe. Eine mögliche Lösung besteht darin, unter Sauerstoffgegendruck bzw. unter Luft zu tempern. In diesem Fall kann allerdings Wasserstoff von der Oberfläche in den Kristall diffundieren oder zumindest bleibt der Wasserstoff, der sich wachstumsbedingt im Kristall befindet, dort. Da Wasserstoff in Zinkoxid als Donator agiert[Wal00], ist es wahrscheinlich, dass der Wasserstoff durch eine Coulombwechselwirkung die Stickstoffakzeptoren neutralisiert, indem sich N-H-Paare bilden. Es konnte in diesem Zusammenhang beobachtet werden, dass ein identisch präparierter Kristall, der unter Luft statt im Vakuum getempert wurde, eine wesentlich geringere Intensität der DAPBande zeigt. Dies ist außerdem eine mögliche Erklärung für die geringe Intensität der DAP-Bande in Nanodrähten, wo eine potentielle Eindiffusion des Wasserstoffs nach dem Tempern durch kurze Diffusionswege in Nanomaterialien schneller erfolgen kann. Eine Möglichkeit, die Effizienz der Ionenstrahldotierung dennoch zu erhöhen besteht darin, die Implantation bei höheren Temperaturen durchzuführen. Eine höhere Temperatur während der Implantation führt durch einen dynamischen Ausheilprozess zu einer starken Verringerung der zurückbleibenden Kristalldefekte, wodurch eine anschließende Temperaturbehandlung überflüssig wird. Ursache dieses Effekts ist, dass die Energie der eintreffenden Ionen bei höheren Temperaturen langsamer dissipiert. Dies führt dazu, dass Defekte instantan ausheilen können [McC95].

Abbildung 3.17 zeigt in logarithmischer Auftragung Lumineszenzspektren von Zinkoxid-Kristallen, die bei verschiedenen Temperaturen $\left(300{ }^{\circ} \mathrm{C}\right.$ und $\left.500{ }^{\circ} \mathrm{C}\right) \mathrm{im}$ plantiert wurden, zusammen mit dem Spektrum eines Kristalls, der bei Raumtemperatur implantiert und anschließend bei $600{ }^{\circ} \mathrm{C}$ getempert wurde. Die Anregung erfolgte bei moderater Anregungsdichte bei 3,815 eV und einer Temperatur von 13 K. Wie erwartet nimmt die Intensität der DAP-Bande sowie derer Phononenwiederholer mit steigender Implantationstemperatur zu. Die Phononenwiederholer höherer Ordnung sind bei höheren Implantationstemperaturen nicht mehr als individuelle Banden im Lumineszenzspektrum zu erkennen. Dies ist möglicherweise darauf zurückzuführen, dass die verwendeten Proben aus unterschiedlichen Kristalen geschnitten wurden. Es ist bekannt, dass die Kristalle eine unterschiedliche Hinter- 
grundkonzentration von Verunreinigungen, insbesondere durch Übergangsmetalle, haben [Cry]. Übergangsmetalle zeigen eine starke phononische Kopplung in Zinkoxid was schließlich zu einer Verbreiterung der Phononenwiederholer führen kann. Die Intensität der DAP-Bande ist, obgleich sie durch diesen Prozess deutlich erhöht werden konnte, noch immer mehr als eine Größenordnung kleiner als die der donatorgebundenen Exzitonen.

\subsection{Zusammenfassung}

In diesem Kapitel wurden Ergebnisse systematischer optischer Untersuchungen an Zinkoxid-Nanodrähten gezeigt, die in Göttingen bzw. in verschiedenen Kollaborationen hergestellt wurden. Es wurde gezeigt, dass die Lumineszenz von Nanodrähten mit einem Durchmesser von einigen $100 \mathrm{~nm}$ bei tiefen Temperaturen durch donatorgebundenen Exzitonen und deren Phononenwiederholer und bei Raumtemperatur durch freie Exzitonen und deren Phonenwiederholer geprägt ist. Damit unterscheidet sich die Lumineszenz dieser Nanodrähte nicht von Volumenkristallen. Auch die Halbwertsbreite der Linien gebundener Exzitonen ist vergleichbar mit hochwertigen Volumenkristallen, was auf eine hohe Kristallqualität der vergleichsweise einfach hergestellten Drähte hinweist. Weiterhin konnte gezeigt werden, dass sich das Lumineszenzspektrum eines Nanodrahtensembles nicht wesentlich vom Spektrum einzelner Drähte unterscheidet, was auf ein homogenes Wachtum der Nanodrähte über die Probenfläche hinweist.

An dünneren Zinkoxid-Nanodrähten konnten spezifische Eigenschaften der Nanodrähte gezeigt werden. (1) Effekte der Oberfläche, die aufgrund der Geometrie bei kleiner werdendem Durchmesser relativ gesehen zunimmt, (2) Effekte der dimensionalen Begrenzung der Elektronen-Lochpaare, was zu einem quantum confinement führt und (3) Wellenleitereffekte, wodurch es zu einer Ausbildung bestimmter optischer Moden kommt.

Es wurde im Detail gezeigt, dass eine Bande bei 3,366 eV bei kleiner werdenden Strukturen an relativer Intensität gewinnt. Diese Bande wurde bereits in der Literatur als Oberflächeneffekt (1) behandelt, was durch die Ergebnisse im Rahmen der Untersuchungen unterstützt werden kann. An sehr dünnen Zinkoxid-Nanodrähten mit einem Durchmesser von nur $\approx 4 \mathrm{~nm}$ konnte indirekt, durch blauverschoben 
auftretende Phononenwiederholer, ein quantum confinement-Effekt (2) nachgewiesen werden, der vergleichbar bisher nur bei Zinkoxid-Quantenpunkten beobachtet wurde. Eine Bande, die bei größeren Zinkoxid-Strukturen unterhalb von 3,33 eV auftritt und von der gezeigt wurde, dass sie auf inelastische Exziton-ExzitonStreuung zurückzuführen ist, tritt bei dünneren Drähten nicht auf. Dies wurde darauf zurückgeführt, dass die Anzahl der Zustände, die durch einen Exziton-ExzitonStreuprozess besetzt würden, aufgrund der Form der Nanodrähte stark reduziert ist. Da der Wirkungsquerschnitt für einen solchen Streuprozess auch von der Zustandsdichte unbesetzter Endzustände abhängt, wird der Streuprozess effektiv unterdrückt. Die Form der Nanodrähte beeinflusst die Zustandsdichte insofern, als dass sich bei Nanodrähten mit einem Durchmesser unter $130 \mathrm{~nm}$ nur noch eine Mode in diesem Wellenlängenbereich ausbilden kann, sodass Länge und Durchmesser in einer festen Beziehung stehen müssen, damit sich Licht dieser Wellenlänge in ihnen ausbreiten kann. Dies ist statistisch bedingt nur in seltenen Fällen gegeben. Das Ausbleiben der Bande bei 3,33 eV als makroskopische Eigenschaft konnte folglich auf die Wellenleiterstruktur (3) der Nanodrähte zurückgeführt werden.

Neben den Untersuchungen an den gewachsenen Strukturen wurden diese auch gezielt verändert. Es wurde Stickstoff in die Nanodrähte implantiert, der, optisch aktiviert, zu einem Donator-Akzeptor-Paarübergang bei $\approx 3,230 \mathrm{eV}$ im Lumineszenzspektrum geführt hat. Dieser wurde, da er in der Literatur kontrovers diskutiert wurde, in Untersuchungen an Volumenkristallen eindeutig als solcher identifiziert. 


\section{Kapitel 4}

\section{Modifikation von Halbleiternanodrähten}

Die Veränderung der Eigenschaften von Halbleitermaterialien durch das Einbringen von Fremdatomen ist essentiell für deren Funktionalisierung als elektrische, optoelektronische und photonische Bauteile. Dies gilt für Nanodrähte gleichermaßen wie für dünne Schichten und Volumenkristalle. Da sich der Einbau von Fremdatomen in Nanodrähte während des Wachstums aufgrund des Wachstumsmechanismus als besonders schwierig erweist, besteht eine vielversprechende Alternative in dem nachträglichen Einbringen durch Ionenimplantation. Neben den vielen Vorteilen dieses Prozesses führt die Ionenimplantation nahezu immer zu einer strukturellen Veränderung des Materials. Zum einen erzeugen die energetischen Ionen Defekte im Kristall, zum anderen kommt es zu einem Abtrag von Oberflächenatomen, was als Sputtereffekt bezeichnet wird (vgl. Abschnitt 2.2.1). Beide Effekte hängen stark von den experimentellen Gegebenheiten ab, beispielsweise vom verwendeten Halbleitermaterial, der Ionenart, -energie und -fluenz.

In den folgenden beiden Abschnitten werden Untersuchungen bezüglich der strukturellen Veränderungen an zwei Systemen präsentiert, die sich hinsichtlich dieser beiden Effekte stark unterscheiden. Bei dem ersten System handelt es sich um ZinkoxidNanodrähte, die mit schweren Ionen (Seltenen Erden) und hoher Fluenz bestrahlt wurden, mit dem Ziel, die Seltenen Erden optisch zu aktivieren (siehe Kapitel 5). Bei dem zweiten System handelt es sich um epitaktisch gewachsene GalliumarsenidNanodrähte, die mit einer moderaten Fluenz von Zinkionen implantiert wurden, mit dem Ziel einer elektrischen Dotierung des Halbleiters (siehe Kapitel 6). 


\subsection{Strukturelle Veränderungen an Zinkoxid- Nanodrähten durch Ionenimplantation}

Das Material Zinkoxid verhält sich relativ unempfindlich gegenüber der Bestrahlung durch energetische Teilchen. Dies deutete sich bereits in Abschnitt 3.7 an, wo gezeigt wurde, dass die bandkantennahe Lumineszenz von Zinkoxid-Kristallen nach einer Implantation mit Stickstoff nahezu unverändert bleibt. Diese Strahlenhärte würde Zinkoxid basierte Bauteile potenziell interessant für den Einsatz in strahlenbelasteten Bereichen, wie zum Beispiel dem Weltraum, machen. Die Strahlenhärte des Materials ist insbesondere auf die große Ionizität der Bindung von 0,62 auf der Phillipsskala zurückzuführen [Nag75]. Nach dem Kriterium der Bindungsart amorphisieren keine Substanzen mit einer Ionizität größer als 0,47 [Nag75]. In der Literatur wurde die Strahlenhärte insbesondere durch die Bestrahlung mit leichten, hochenergetischen Teilchen wie Protonen [Aur01] und Elektronen [Loo99] untersucht. Im Folgenden werden Untersuchungen an dünnen Zinkoxid-Nanodrähten gezeigt, die mit schweren Elementen (Seltenen Erden) und hoher Dosis bestrahlt wurden. Es werden zunächst die strukturellen Veränderungen durch den Sputtereffekt, anschließend die Veränderung des Kristallgitters betrachtet.

\subsubsection{Präparation der Proben}

Im Experiment wurden Zinkoxid-Nanodrähte mit einem mittleren Durchmesser von $\approx 40 \mathrm{~nm}$ mit Seltenen Erden und Xenon bestrahlt. Die in der Kollaboration Li/Hong Kong hergestellten Drähte wurden dafür zunächst mit hoher Dichte auf mehrere saubere Siliziumsubstrate übertragen, wie es in Abschnitt 2.2.2 beschrieben ist. Abbildung 3.13 c) zeigt ein typisches SEM-Bild der präparierten Proben. Die Implantationsparameter der zwölf untersuchten Proben sind in Tabelle 4.1.1 aufgeführt.

\begin{tabular}{c|cccc}
$\begin{array}{c}\text { Probenbezeichnung } \\
\mathrm{Eu}_{-} \mathrm{Tm}_{-} \mathrm{Yb}_{-} \mathrm{Xe}_{-}\end{array}$ & $\begin{array}{c}\text { Energie } \\
\mathrm{keV}\end{array}$ & $\begin{array}{c}\text { Gesamtfluenz } \\
\mathrm{cm}^{-2}\end{array}$ & $\begin{array}{c}\text { Konzentration } \\
\text { at. } \mathrm{cm}^{-3}\end{array}$ & $\begin{array}{c}\text { Konzentration } \\
\text { at. \% }\end{array}$ \\
\hline 1 & $5-55$ & $5,2 \cdot 10^{14}$ & $2,7 \cdot 10^{20}$ & 0,3 \\
2 & $5-55$ & $1,7 \cdot 10^{15}$ & $8,8 \cdot 10^{20}$ & 1,1 \\
3 & $5-55$ & $5,2 \cdot 10^{15}$ & $2,7 \cdot 10^{21}$ & 3,3
\end{tabular}

Tabelle 4.1: Probenbezeichnung und Implantationsparameter der untersuchten Proben. 

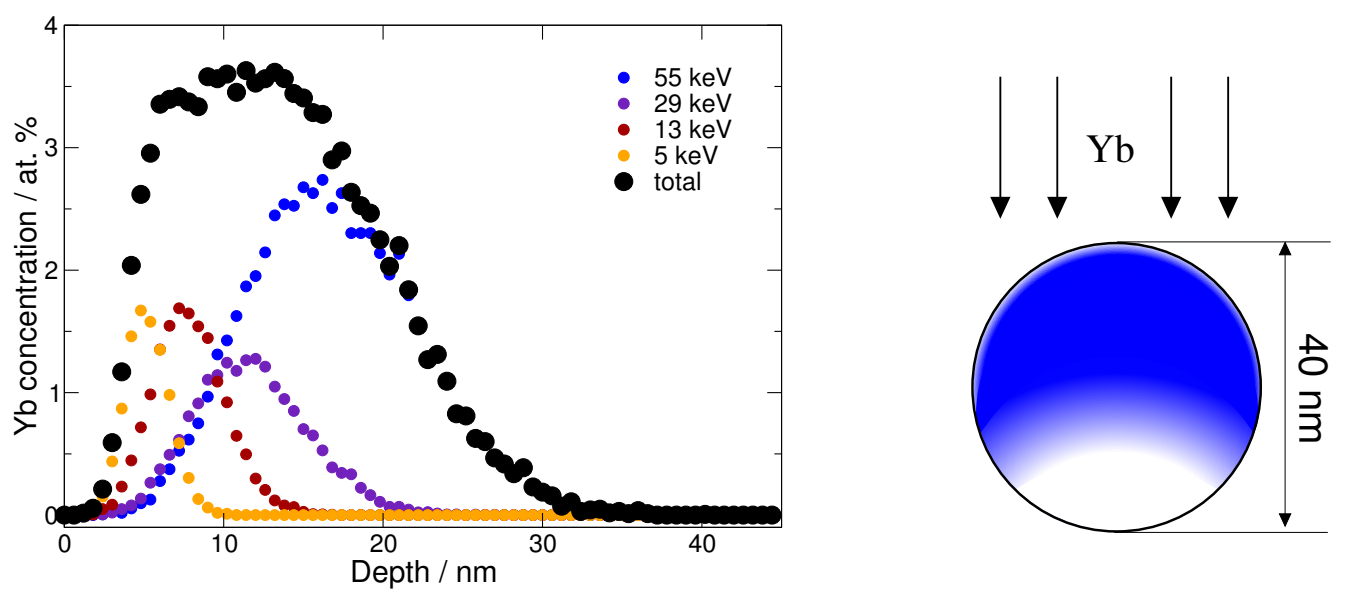

Abbildung 4.1: Implantationsprofil der Seltenen Erden in Zinkoxid-Nanodrähten exemplarisch für die Probe Yb_3. Die Ionenreichweite wurde für vier verschiedene Energien mit TRIM berechnet. Die schwarzen Punkte zeigen das resultierende Konzentrationsprofil.

Es wurden vier verschiedene Ionenspezies (Europium, Thulium, Ytterbium und Xenon) mit drei verschiedenen Fluenzen implantiert. Um in den Nanodrähten einen Bereich mit homogener Konzentration der implantierten Spezies zu erzeugen, wurden Ionen mit vier verschiedenen Energien zwischen 5 und $55 \mathrm{keV}$ verwendet. Das resultierende Implantationsprofil, wie es zuvor mit TRIM berechnet wurde, ist exemplarisch für die Probe Yb_3 in Abbildung 4.1 dargestellt. Die oberen $20 \mathrm{~nm}$ des Nanodrahtes haben demnach eine homogene Ytterbiumkonzentration von 3,3 at.\%. Im rechten Teil der Abbildung ist schematisch die Implantationsbedingung für einen Nanodraht mit einem Durchmesser von $40 \mathrm{~nm}$ im Querschnitt dargestellt. Die blaue Einfärbung stellt dabei die berechnete Konzentration der Seltenen Erden dar. Nach der Implantation wurden die Proben für 30 min bei $700{ }^{\circ} \mathrm{C}$ unter Sauerstoff-Gasfluss getempert.

\subsubsection{Sputtereffekte durch lonenbestrahlung}

Abbildung 4.2 zeigt SEM-Aufnahmen von Zinkoxid-Nanodrähten, die mit verschiedenen Fluenzen Seltener Erden bestrahlt und anschließend bei $700{ }^{\circ} \mathrm{C}$ getempert wurden. Mit zunehmender Ionenfluenz ist eine deutliche Veränderung der Morphologie zu beobachten. Bereits bei der geringsten Dosis sind lokale Vertiefungen in den 
0.3 at. $\%$
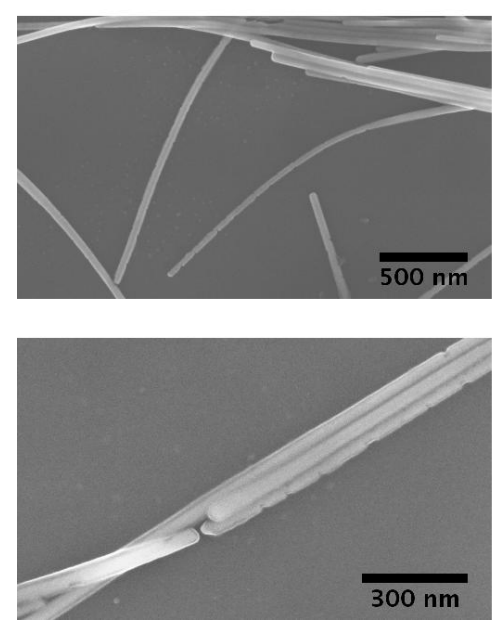

1.1 at. $\%$
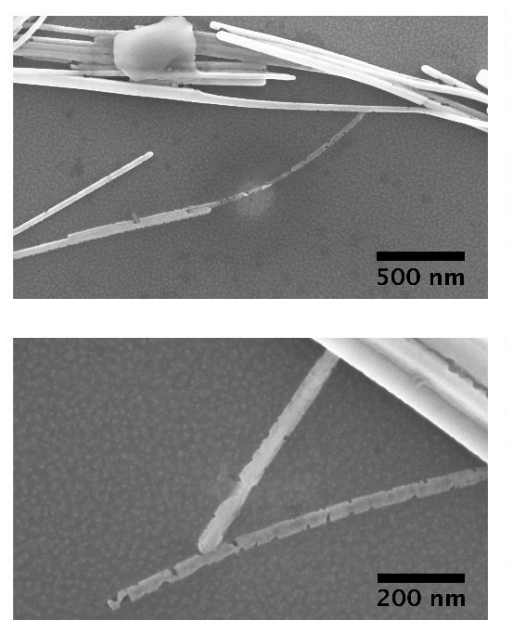

3.3 at. $\%$
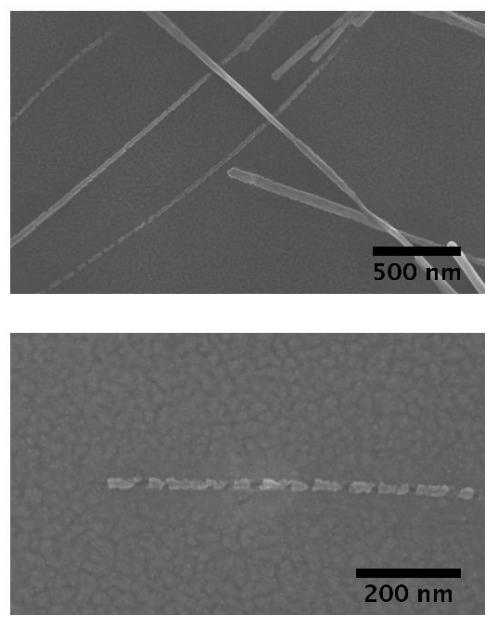

Abbildung 4.2: SEM-Aufnahmen von Zinkoxid-Nanodrähten, die mit verschiedenen Fluenzen von Seltenen Erden bestrahlt wurden. Von links nach rechts sind steigende Fluenzen aufgetragen. Die untere Reihe zeigt SEM-Aufnahmen mit höherer Vergrößerung.

Nanodrähten zu beobachten, wobei die Oberflächen der Drähte noch relativ eben wirkt. Bei steigender Fluenz raut die Oberfläche zunehmend auf; auch starke Vertiefungen werden häufiger und sind deutlicher ausgeprägt. Bei hoher Fluenz sind die Nanodrähte an den Stellen der Vertiefungen zum Teil sogar zertrennt. Diese Beobachtungen sind unabhängig von der Spezies der Seltenen Erden und auch an den mit Xenon bestrahlten Nanodrähten zu beobachten.

Die strukturelle Veränderung der Drähte lässt sich noch deutlicher in SEMAufnahmen unter flachem Winkel erkennen, wie sie für die Proben Yb_2 und Yb_3 in Abbildung $4.3 \mathrm{zu}$ sehen sind. Im Mittel werden die Drähte mit zunehmender Ionenfluenz dünner, was ein Vergleich der Abbildungen 4.3 a) und c) zeigt. Außerdem nimmt der Grad der strukturellen Veränderung zu. Bei stärkerer Vergrößerung (vgl. Abbildung 4.3 b)) deutet sich an, dass die Nanodrähte keine runde Querschnittsform mehr haben. Die Substratoberfläche ist entlang der Nanodrähte nach oben gewölbt, sodass die Nanodrähte exponiert auf Inseln liegen.

Die strukturelle Veränderung der Nanodrähte durch den Ionenbeschuss ist auf das Abtragen von Oberflächenatomen zurückzuführen. Dieser Prozess wird durch den Sputtereffekt beschrieben (vgl. Abschnitt 2.2.1). Die mittlere Anzahl abge- 
1.1 at. $\%$
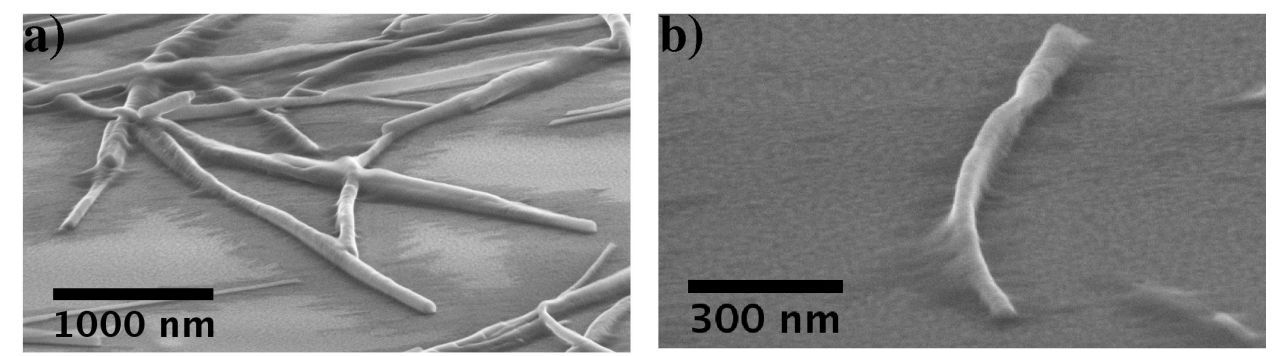

3.3 at. $\%$
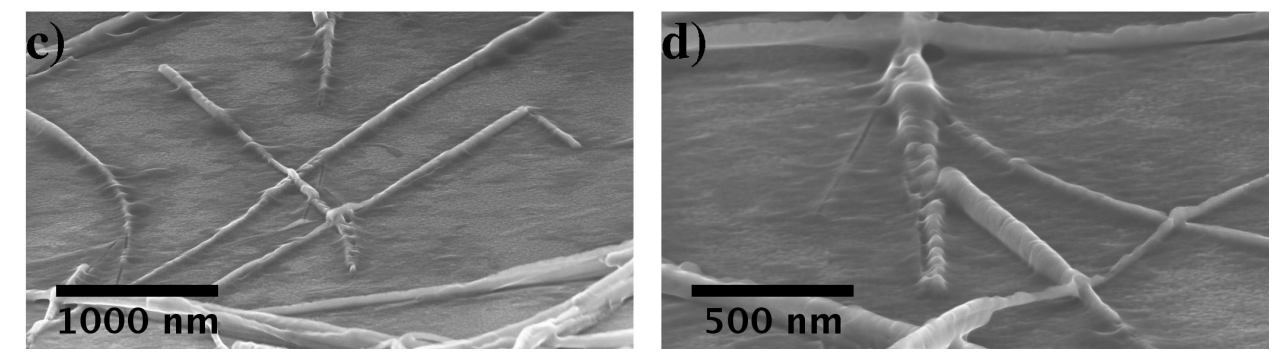

Abbildung 4.3: SEM-Aufnahmen unter flachem Winkel von Zinkoxid-Nanodrähten, die mit verschiedenen Fluenzen von Seltenen Erd-Ionen bestrahlt wurden. a) und b) zeigen Aufnahmen der Probe Yb_2, c) und d) der Probe Yb_3.

tragener Oberflächenatome pro einfallendem Ion (Sputterkoeffizient) lässt sich mit TRIM simulieren und beträgt beispielsweise für Ytterbiumionen mit einer Energie von $55 \mathrm{keV}$ bei senkrechtem Einfallswinkel $\approx 12$. Bei der höchsten verwendeten Fluenz von $5,2 \cdot 10^{15} \mathrm{~cm}^{-2}$ führt dieser Sputterkoeffizient zu einem Abtrag von $6,2 \cdot 10^{16}$ at. $\mathrm{cm}^{-2}$, was etwa 60 Monolagen entspricht. Dies sind entlang der c-Richtung eines Zinkoxid-Kristalls etwa $30 \mathrm{~nm}$, was zu der Annahme führt, dass dünne Nanodrähte durch die Bestrahlung bereits vollständig abgetragen sind. Tatsächlich sind auf den Siliziumsubstraten inselartige Terrassen zu erkennen, die vermutlich auf eine Abschattung des Substrats durch bereits vollständig abgetragene Nanodrähte zustande gekommen sind (vgl. Abbildung 4.3 d)).

Der Sputterkoeffizient hängt von vielen Parametern ab, unter anderem von der Masse der eintreffenden Ionen und der Targetatome, von der Ionenenergie, dem Einfallswinkel und der Targettemperatur [Kim07a]. Die Ionenmassen im Experiment variieren nicht sehr stark und das Targetmaterial ist identisch, sodass hier keine starke Abhängigkeit erwartet wird. Für die verwendeten Energien sind die Sputterkoeffizienten für Ytterbium, Europium und Xenon bei senkrechtem Einfall in Abbildung 4.4 a) aufgetragen. Der Sputterkoeffizient nimmt mit der Energie der 

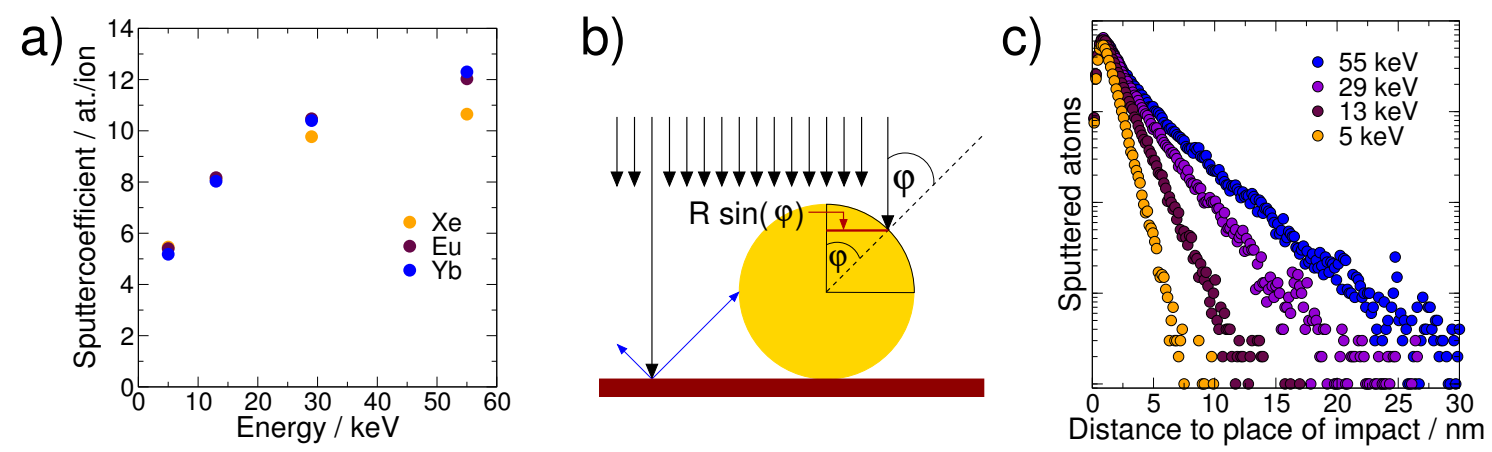

\begin{abstract}
Abbildung 4.4: a) Sputterkoeffizient von Ytterbium, Europium und Xenon auf Zinkoxid bei senkrechtem Einfall als Funktion der Ionenenergie. b) Schema zum Sputterprozess. c) Statistische Verteilung des Abstands abgetragener Oberflächenatome vom Ort, an dem das verursachende Ion in den Festkörper einschlug. Die Grafik zeigt die Verteilung für Ytterbiumionen mit vier unterschiedlichen Energien bei senkrechtem Einfallswinkel auf Zinkoxid.
\end{abstract}

Ionen deutlich zu - für schwere Ionen stärker als für leichtere. Zur Berechnung wurden Verlagerungsenergien von $18 \mathrm{eV}$ für Zink und $45 \mathrm{eV}$ für Sauerstoff angesetzt [Loo99, Vec80]. In der Tat unterscheiden sich die Sputterkoeffizienten der Ionenspezies nur geringfügig. Dies erklärt die Beobachtung, dass alle Proben, die mit der gleichen Fluenz unterschiedlicher Ionen (Xenon, Europium, Thulium, Ytterbium) bestrahlt wurden, ähnliche strukturelle Veränderungen zeigen.

Eine Betrachtung der Implantationsbedingungen zeigt, dass der Einfallswinkel $\varphi$ der Ionen auf dem Nanodraht ortsabhängig ist (vgl. Abbildung 4.4 b)). Mit TRIM lässt sich der Sputterkoeffizient nur für ebene Schichten simulieren. Dabei zeigt sich, dass der mittlere Abstand zwischen den abgetragenen Atomen und dem Ort, an dem das Ion eingetroffen ist, klein gegenüber der Ionenreichweite und dem Drahtdurchmesser ist. Abbildung $4.4 \mathrm{c}$ ) zeigt eine Statistik über diesen Abstand für senkrecht einfallende Ytterbiumionen unterschiedlicher Energie auf einer logarithmischen Skala. Der mittlere Abstand beträgt für Ytterbium-Ionen mit $55 \mathrm{keV}$ etwa 3,4 nm. Bei nicht senkrechtem Einfallswinkel ist die Verteilung abgetragener Atome nicht mehr symmetrisch um den Ort, an dem das Ion eintrifft, sondern entlang der projizierten Einfallsrichtung verbreitert. In jedem Fall führen nur oberflächennahe Kollisionskaskaden zu einem Sputterprozess [Zie08]. Für den vorliegenden Fall gilt nach Sigmund, dass die Dicke einer Schicht, die essenziell zu dem Sputterprozess beiträgt, kleiner als die halbe mittlere Ionenreichweite ist [Sig69]. Dies spricht dafür, dass die mit 
TRIM berechneten Sputterkoeffizienten lokal auf Nanodrähte zumindest dann anwendbar sind, wenn die Ionenreichweite nicht deutlich größer als der Radius der Drähte gewählt wird.

Die Änderung der Oberflächentopographie ist ein stark dynamischer Prozess und auch die Zusammensetzung des Kristalls ändert sich durch die eingebrachten Ytterbiumionen. Beide Faktoren beeinflussen wiederum den Sputterkoeffizienten [Kim07a]. Die Änderung der Zusammensetzung des Kristalls wird in der Softwareentwicklung TRIDYN [Moe02] berücksichtigt, was im Vergleich zu TRIM zu einer deutlich besseren Übereinstimmung zwischen experimentell gemessenem und simuliertem Sputterkoeffizienten führt [Kim07a]. Die dynamische Veränderung der Oberflächentopologie wird neben dem Sputterprozess selbst auch merklich durch die Redeposition abgetragener Oberflächenatome geprägt; in dem betrachteten Fall zusätzlich durch die Redeposition abgetragener Siliziumatome des Trägersubstrats (vgl. Abbildung 4.4 b)). Beide Prozesse sind in der Softwareentwicklung AMADEUS 3D [Kim07a, Kim07b] berücksichtigt, die allerdings nicht frei zugänglich ist.

Im Folgenden wird in einer ersten Näherung angenommen, dass die Sputterkoeffizienten aus TRIM auf die Nanodrähte übertragbar sind. Weiterhin wird angenommen, dass die Nanodrähte ursprünglich eine kreisförmige Querschnittsfläche haben. Die dynamische Veränderung der Oberflächentopographie wird darauf reduziert, dass jeder Ort auf der Oberfläche von einem Ion getroffen wird und dadurch der berechnete Sputterkoeffizient an diesem Ort abgetragen wird.

In Abbildung 4.5 a) ist der Sputterkoeffizient von Ytterbiumionen mit unterschiedlicher Energie als Funktion des Ortes $x \cdot R_{0}$ aufgetragen, wie er mit TRIM berechnet wurde. Nach einem monotonen Anstieg des Sputterkoeffizienten folgt bei großem $x\left(x=0,985 \cdot R_{0}, \varphi \approx 80^{\circ}\right)$ ein Maximum. Anschließend fällt der Sputterkoeffizient steil ab, was vorwiegend auf eine zunehmende Anzahl von elastisch gestreuten Primärionen bei großen $\varphi$ zurückzuführen ist. Für kleine Einfallswinkel $\varphi$ lässt sich der Sputterkoeffizient Y beschreiben durch [Sig69]

$$
Y(\varphi)=a \cdot(\cos (\varphi))^{-b}
$$

Die durchgezogene Linie in Abbildung 4.5 a) zeigt eine Anpassung an die mit TRIM berechneten Werte für $55 \mathrm{keV}$ Ytterbium. Es ergibt sich mit a=12,5 At./Ion und $\mathrm{b}=2,1$ eine gute Übereinstimmung zwischen den berechneten Werten und der An- 
a)

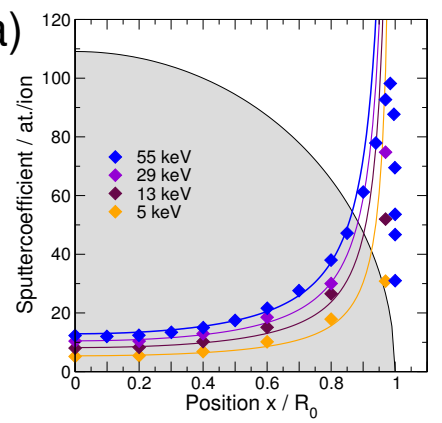

b)

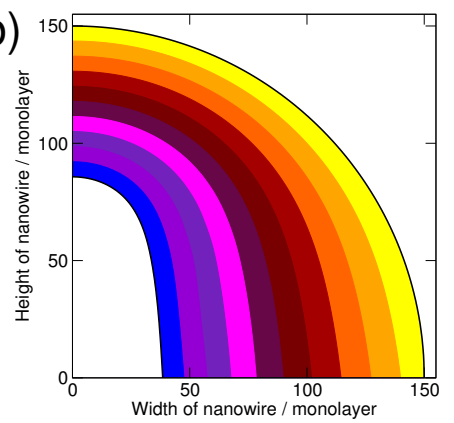

c)

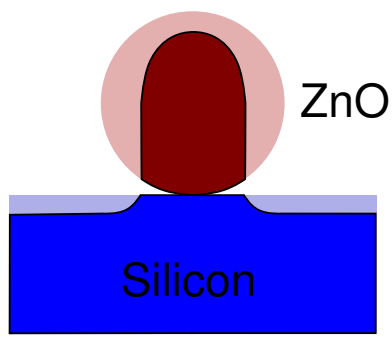

Abbildung 4.5: a) Sputterkoeffizient von Ytterbium auf Zinkoxid als Funktion des Ortes, an dem das Ion auf dem Nanodraht einschlägt. Die durchgezogene Linie wurde mit Gleichung 4.1 angepasst.b) Veränderung der Querschnittsfläche durch iterative Beschreibung des Sputterprozesses am Beispiel eines Kreises (oberer Quadrant). Farbig gekennzeichnet ist die Querschnittsfläche nach jeweils einem Iterationsschritt. c) Schematische Darstellung der erwarteten strukturellen Veränderung von Nanodraht und Substrat im Querschnitt.

passung im Bereich von $x=0$ bis $x=0,85$. Da bei den betrachteten Drähten die Anzahl der Atomlagen bei großem $x$ vergleichbar mit dem berechneten Sputterkoeffizienten ist, wird die Abweichung zunächst vernachlässigt. Dies ist auch gerechtfertigt, wenn es das Ion an dieser Stelle die Probe durchstrahlt, da der Sputterkoeffizient insbesondere durch oberflächennahe Kollisionskaskaden bestimmt wird [Sig69]. Nun lässt sich Gleichung 4.1 verwenden, um, wie oben beschrieben, iterativ die dynamische Veränderung des Drahtquerschnitts zu berechnen. Ein Iterationsschritt entspricht dann der Bestrahlung mit einer Monolage, also etwa mit $5 \cdot 10^{14}$ Ionen $/ \mathrm{cm}^{2}$. Die Form $\bar{F}$ des Drahtes nach einem Iterationsschritt ergibt sich $\mathrm{zu}$

$$
\bar{F}(x)=F(x)-Y(\varphi), \quad \varphi=\arctan \frac{d F}{d x} .
$$

Dabei bezeichnet F die Form vor dem Iterationsschritt und Y ist gegeben durch Gleichung 4.1. Abbildung 4.5 b) zeigt nun, wie sich die Querschnittsform eines Kreises mit einem Durchmesser von 300 Monolagen ( $\approx 90 \mathrm{~nm}$ ) in 10 Iterationsschritten (in etwa die Fluenz der Probe Yb_3) verändert.

Wie nach Abbildung 4.5 a) zu erwarten ist, kommt es zu einer Vergrößerung der Flankensteilheit, so dass die Querschnittsform des Nanodrahtes durch die Ionenbestrahlung zunehmend rechteckig/oval wird. Abbildung $4.5 \mathrm{c}$ ) zeigt nun schematisch, wie sich entsprechend dem Modell die Geometrie des Drahtquerschnitts verändern 


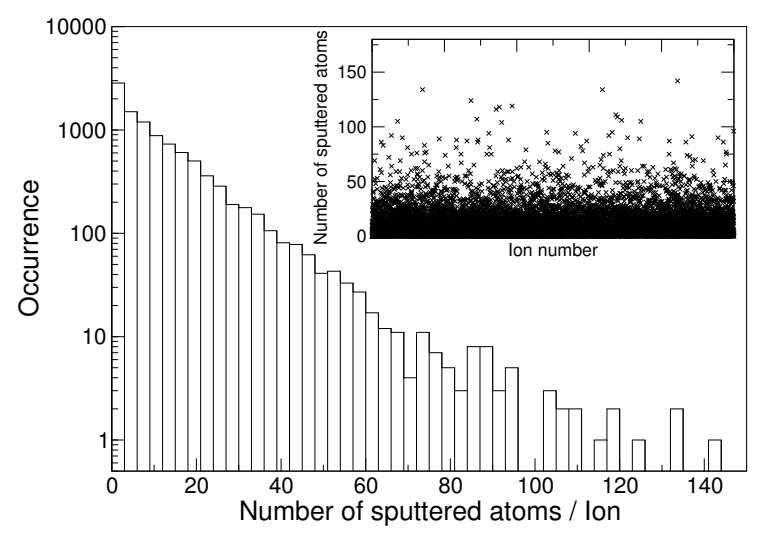

Abbildung 4.6: Statistik über die Sputterkoeffizienten von $55 \mathrm{keV}$ Ytterbiumionen auf Zinkoxid bei senkrechtem Einfall. Die Häufigkeit ist logarithmisch dargestellt. Der Mittelwert liegt bei 12,3 At./Ion. Im Einsatz sind die Sputterkoeffizienten einzelner Ionen aufgetragen.

sollte, wobei die aufgehellten Bereiche die Situation vor der Ionenbestrahlung darstellen. Trotz der starken Näherung ist durchaus eine gewisse Ähnlichkeit mit den beobachteten strukturellen Veränderungen in Abbildung 4.3 b) und d) zu erkennen.

Über die beobachteten Vertiefungen an den Nanodrähten kann diese Betrachtung keinen Aufschluss geben. Hier ist es sinnvoll, einen Blick auf die Verteilung der Sputterkoeffizienten einzelner Ionen zu werfen. Dies ist im Einsatz von Abbildung 4.6 für $55 \mathrm{keV}$ Ytterbium-Ionen bei senkrechtem Einfall aufgetragen. Es zeigt sich, dass der Sputterkoeffizient durchaus stark variieren kann. Obgleich der mittlere Sputterkoeffizient etwa 12 at./Ion ist, können einzelne Ionen durchaus bis zu 140 Atome aus dem Festkörper herausschlagen. Dies führt dazu, dass sich die Oberflächenkrümmung lokal vergrößert, was wiederum zu einem höheren mittleren Sputterkoeffizienten führt. Wenn also durch einen solchen Ioneneinschlag eine Vertiefung entstanden ist, so wird diese größer werden. Eine Häufigkeitsstatistik über die Sputterkoeffizienten von 10000 Ionen zeigt Abbildung 4.6 auf einer logarithmischen Skala. In etwa jedes 1000ste Ion hat einen um Faktor 10 größeren Sputterkoeffizienten als den Mittelwert. Zusätzlich führt der starke ionische Bindungsanteil in Zinkoxid dazu, dass das Material bei Ionenbestrahlung nicht amorphisiert [Nag75], was die Untersuchungen im nächsten Abschnitt zeigen. Entsprechend kann auch die ständige instantane Rekristallisation während einer Ionenbestrahlung zur Ausformung lokaler Vertiefungen 

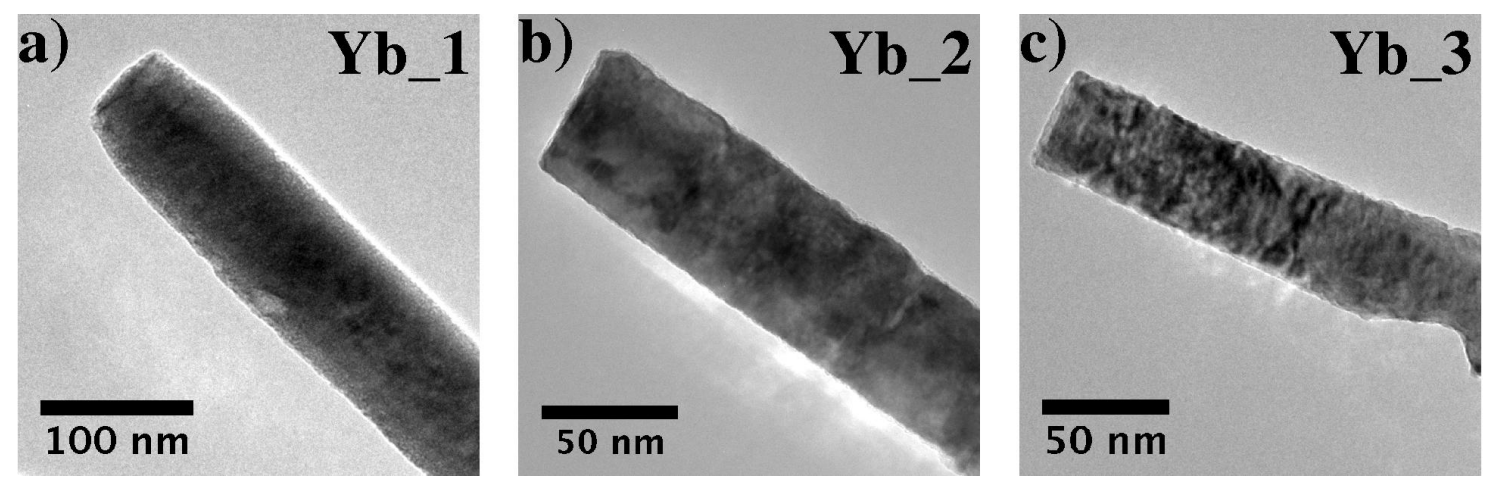

Abbildung 4.7: Hellfeld TEM-Aufnahmen von Zinkoxid-Nanodrähten, die mit unterschiedlichen Fluenzen von Ytterbium implantiert wurden. a) Proben Yb_1, bestrahlt mit einer Dosis von $\left.5,2 \cdot 10^{14} \mathrm{~cm}^{-2}, \mathbf{b}\right)$ Proben Yb_2, bestrahlt mit einer Dosis von $1,7 \cdot 10^{15} \mathrm{~cm}^{-2}$ und c) Proben Yb_3, bestrahlt mit einer Dosis von $5,2 \cdot 10^{15} \mathrm{~cm}^{-2}$.

an der Oberfläche beitragen. Ein Anhaltspunkt dafür ist, dass die Vertiefungen eine charakteristische, sägezahnartige Form haben, wie es in Abbildung 4.2 zu sehen ist.

\subsubsection{Strukturelle Veränderung des Kristallgitters durch lonen- implantation}

Um die strukturelle Veränderung des Kristallgitters und der Oberfläche, sowie den Einbau der Seltenen Erden in das Kristallgitter näher zu untersuchen, wurden TEM-Untersuchungen an den implantierten und getemperten ZinkoxidNanodrähten durchgeführt. Abbildung 4.7 zeigt TEM-Hellfeldaufnahmen von Zinkoxid-Nanodrähten mit vergleichbarem Durchmesser, die mit unterschiedlichen Fluenzen von Ytterbium implantiert wurden. Die verwendete Ionenfluenz steigt von links nach rechts von 5,2 $10^{14} \mathrm{~cm}^{-2}$ für die Probe Yb_1 auf 5,2 $10^{15} \mathrm{~cm}^{-2}$ für die Probe Yb_3, was entsprechend den Implantationsparametern YtterbiumKonzentrationen von 1,1 at.\% bis 3,3 at.\% entspricht. Abbildung 4.8 zeigt analog hochaufösende TEM-Aufnahmen mit den zugehörigen Fouriertransformationen (FFT).

Aus den Untersuchungen ergeben sich im Wesentlichen drei wichtige Ergebnisse. (1) Sowohl die Hellfeldaufnahmen als auch die hochauflösenden Aufnahmen bestätigen die Beobachtungen einer zunehmenden Aufrauung der Nanodrahtoberfläche mit steigender Ionenfluenz. Bei der geringsten Ytterbiumdosis (vgl. Abbildung 4.7 a)) 

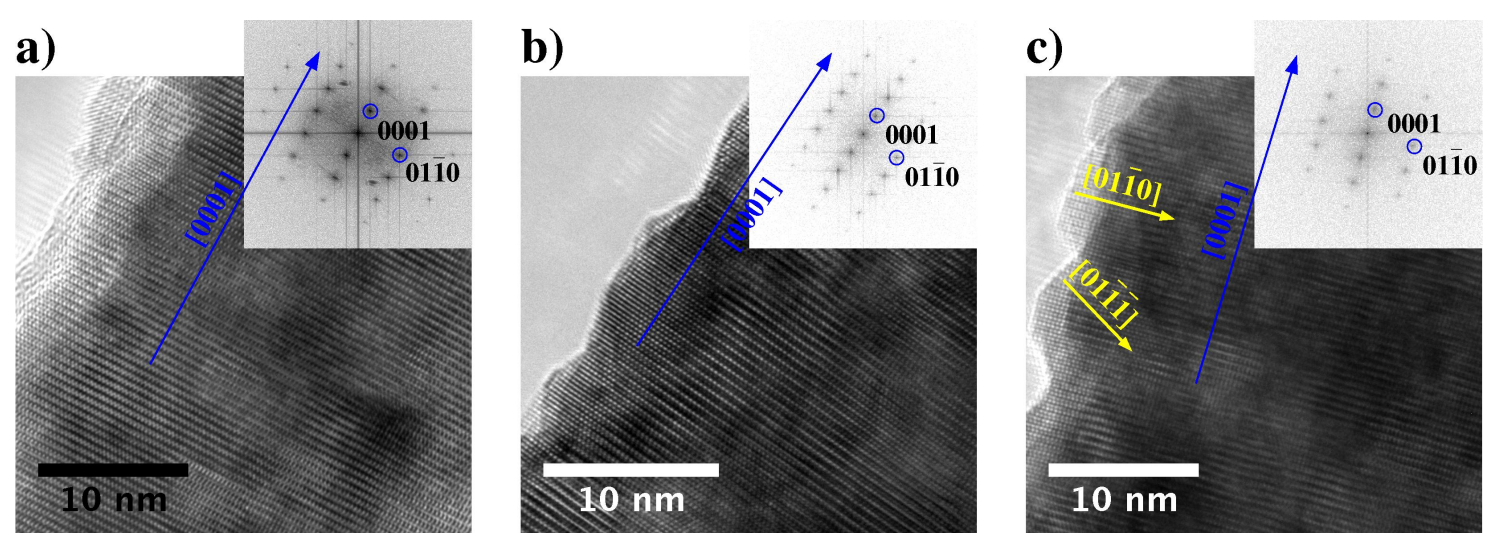

Abbildung 4.8: Hochaufösende TEM-Aufnahmen mit FFT von Zinkoxid-Nanodrähten, die mit unterschiedlichen Fluenzen von Ytterbium implantiert wurden. a) Proben Yb_1, bestrahlt mit einer Dosis von $5,2 \cdot 10^{14} \mathrm{~cm}^{-2}$, b) Proben Yb_2, bestrahlt mit einer Dosis von $1,7 \cdot 10^{15} \mathrm{~cm}^{-2}$ und c) Proben $\mathrm{Yb} \_3$, bestrahlt mit einer Dosis von $5,2 \cdot 10^{15} \mathrm{~cm}^{-2}$. Die Wachstumsrichtung entspricht der [0001]-Richtung des Kristalls. Die gelben Pfeile entsprechen den Oberflächennormalen.

ist die Oberfläche der Drähte nahezu glatt, während bei steigender Ionendosis eine zunehmende Aufrauung zu erkennen ist. Bei der höchsten Ionendosis (vgl. Abbildung 4.7 c)) ist außerdem zu erkennen, dass größere Bereiche des Nanodrahtes abgetragen sind, was sich mit den beobachteten Vertiefungen in den SEM-Bildern deckt. Die hochauflösenden TEM-Aufnahmen weisen zusätzlich darauf hin, dass die aufgeraute Oberfläche von niedrig indizierten Kristallebenen abgeschlossen wird, was zu einer Bildung von Facetten führt. Besonders ausgeprägt tritt dies an den Proben mit der höchsten Konzentration auf (vgl. Abbildung 4.8 c) ). Hier sind die entsprechenden Richtungen als gelbe Pfeile in das TEM-Bild eingetragen.

(2) Desweiteren zeigen Hellfeld- und Hochauflösungsaufnahmen lokal stark variierenden Kontrast. Dieser Effekt nimmt ebenfalls mit der Fluenz der Implantation zu. Die Möglichkeit, dass es sich bei den Gebieten, die in der Hochauflösung besonders dunkel erscheinen, um Ausscheidungen Seltener Erden handeln könnte, kann insofern entkräftet werden, dass diese dunklen Bereiche ebenfalls bei der mit Xenon implantierten Referenzprobe auftreten (vgl. Abbildung 4.9 a und b)). Auch EDX-Messungen in diesen Bereichen zeigen keine erhöhten Konzentrationen Seltener Erden. Entsprechend wird angenommen, dass dieser Kontrast in lokalen Dickeänderung bzw. in nicht vollständiger Ausheilung des Gitters begründet ist. 
a)

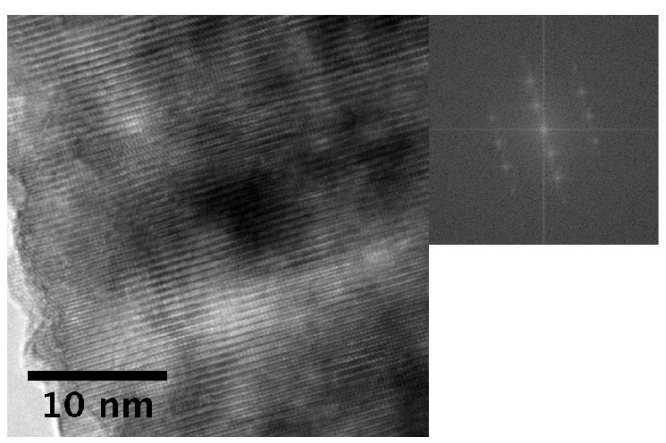

b)

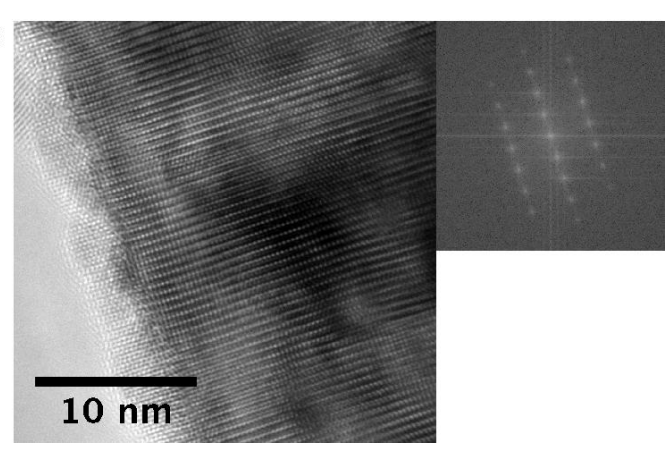

Abbildung 4.9: Hochauflösende TEM-Bilder von Nanodrähten der Proben Yb_3 (a)) und Xe_3(b)). An beiden Proben sind lokal deutlich unterschiedliche Kontraste zu beobachten.

Die hochauflösenden TEM-Aufnahmen zeigen außerdem, dass das Kristallgitter der Nanodrähte nach der Implantation vollständig intakt ist. Die Wachstumsrichtung der Nanodrähte lässt sich aus den FFTs in Übereinstimmung mit der Literatur der [0001]-Richtung des Kristalls zuordnen [Bor06a, Cha04]. Ausgedehnte Defekte des Kristallgitters konnten in diesen Untersuchungen sehr vereinzelt in Form von Stapelfehlern und einzelnen Versetzungen beobachtet werden. Da diese mit zunehmender Dosis nicht häufiger auftreten, wird allerdings vermutet, dass diese wachstumsbedingt und nicht implantationsbedingt sind. Bei Nanodrähten mit größerem Durchmesser kann bei gleichen Bestrahlungsbedingungen beobachtete werden, dass die Seite, die dem Ionenstrahl zugewandt war, die oben beschriebenen Defekte aufweist, während die andere Seite ungeschädigt ist. Ein entsprechendes TEM-Bild ist in Abbildung 4.10 a) dargestellt.

(3) Abbildung 4.10 b) zeigt EDX-Spektren von Zinkoxid-Nanodrähten, die mit verschiedenen Fluenzen von Ytterbiumionen implantiert und anschließend getempert wurden. Die Spektren sind auf einer logarithmischen Skala aufgetragen. Bei der geringsten Fluenz (Yb_1) kann neben den charakteristischen Röntgenlinien von Zink, Sauerstoff und Kupfer (Probenhalter) kein weiteres Element nachgewiesen werden. Bei steigender Fluenz kommt bei 1,6 keV und 7,4 keV eine Linie auf, die der charakteristischen Röntgenstrahlung von Ytterbium zugeordnet werden kann. Die beobachteten Intensitäten entsprechen im Rahmen der Messgenauigkeit den angestrebten Ytterbiumkonzentrationen von $\approx 1$ at.\% für die Probe $Y b \_2$ und $\approx 3$ at.\% für die Probe Yb_3. Es kann folglich davon ausgegangen werden, dass die Seltenen Erden 

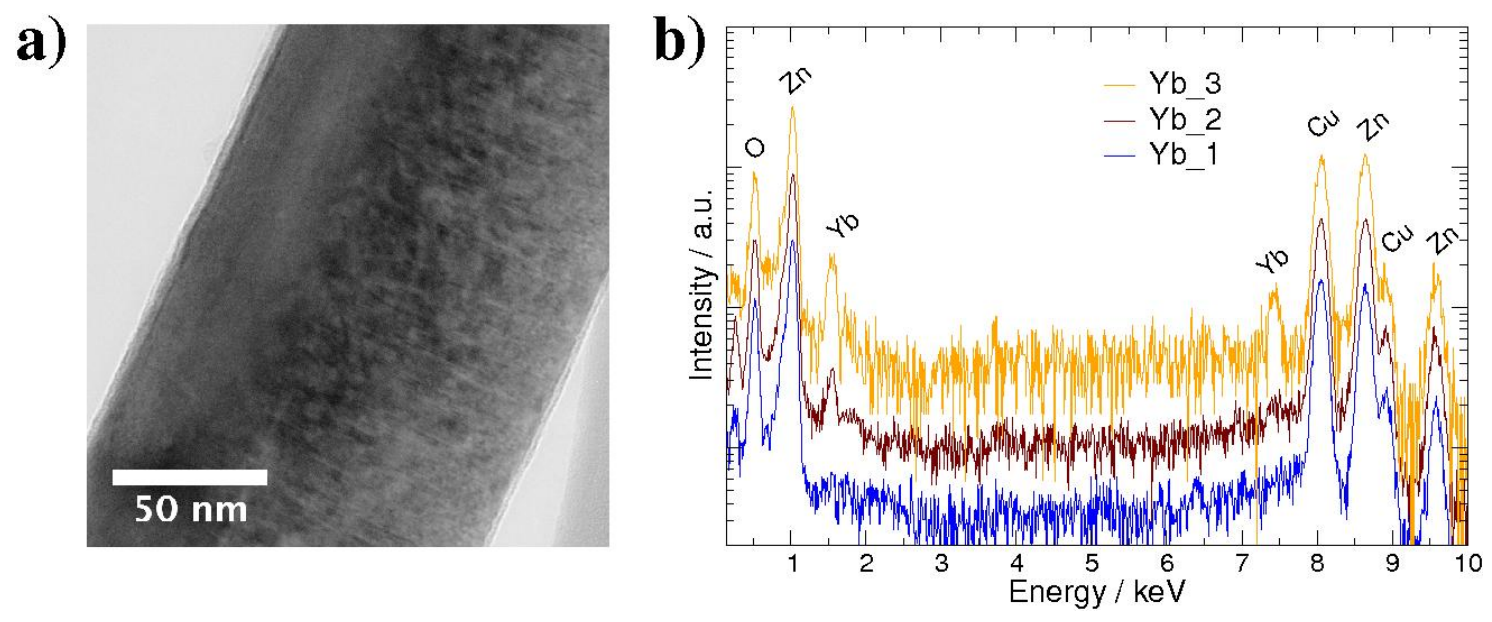

\begin{abstract}
Abbildung 4.10: a) TEM-Hellfeldaufnahmen eines Zinkoxid-Nanodrahtes der Probe Yb_3, dessen Durchmesser etwa $100 \mathrm{~nm}$ beträgt. Der rechte Teil zeigt starke Kontraste, während der linke Teil relativ homogen wirkt. b) EDX-Spektren von Nanodrähten die mit verschiedenen Fluenzen von Ytterbiumionen implantiert und anschließend getempert wurden. Mit steigender Fluenz steigt das Signal der charakteristischen Röntgenlinien des Ytterbiums.
\end{abstract}

auch nach dem Tempern in dem Kristall verbleiben. Diese Beobachtung deckt sich im Wesentlichen mit einem in der Literatur beschriebenem Experiment an Erbiumimplantierten Volumenkristallen [Alv03], bei dem vergleichbare Fluenzen bei der Implantation verwendet wurden $\left(5 \cdot 10^{14} \mathrm{~cm}^{-2}\right.$ und $\left.5 \cdot 10^{15} \mathrm{~cm}^{-2}\right)$. Hier konnte in Rutherford-Rückstreuexperimenten (RBS) gezeigt werden, dass das Erbium nach einem Temperprozess bei $800{ }^{\circ} \mathrm{C}$ vollständig in dem Kristall verbleibt und zwar zu 90\% auf Zink-Gitterplätzen [Rit06]. Analog zu den oben dargestellten TEMErgebnissen konnte auch bei diesen Experimenten nach einer Temperaturbehandlung für 30 min bei $800{ }^{\circ} \mathrm{C}$ mittels RBS-channeling-Messungen noch eine verbleibende Gitterschädigung beobachtet werden. Bei der niedrigen Fluenz wird dieser auf $2 \%$, bei der hohen Dosis auf $22 \%$ beziffert [Alv03]. Nach einer weiteren Temperaturbehandlung bei $1050{ }^{\circ} \mathrm{C}$ wurde beobachtet, dass sich das Gitter vollständig erholt, das Erbium aber zu einem großen Teil ausdiffundiert ist.

Die Arten von Defekten, die in Zinkoxid durch Ionenimplantation entstehen, wird in einigen Publikationen diskutiert [Lor05, Cos04, Aur01, Kuc03]. Zunächst wurde bei Zinkoxid, im Vergleich zu anderen Halbleitern ( $\mathrm{Si}, \mathrm{GaN}$ ), eine deutlich geringere Produktionsrate primärer Defekte (Leerstellen und Zwischengitteratome) durch die 
Bestrahlung mit hochenergetischen Protonen beobachtet [Aur01]. Es konnte gezeigt werden, dass primäre Defekte bei Raumtemperatur mobil sind, während sekundäre Defekte (Doppelleerstellen, Komplexe aus Leerstellen und Verunreinigungen) eine sehr hohe thermische Stabilität besitzen [Aur01, Lor05, Cos04, Alv03]. Die Produktionsrate sekundärer Defekte ist ebenfalls vergleichsweise gering [Aur01]. Eine detaillierte Untersuchung der Schädigung des Kristallgitters von Zinkoxid durch Ionen unterschiedlicher Massen (Erbium, Argon und Stickstoff) als Funktion der Ionenfluenzen wurde in Ref. [Lor05] mittels RBS-channeling durchgeführt. Die Implantationen wurden bei tiefer Temperatur (15 K) durchgeführt, um ein instantanes, thermisch aktiviertes Ausheilen zu verhindern. Es ergeben sich, in Übereinstimmung mit einem Modell der Defektwechselwirkung und Amorphisierung [Hec86], vier charakteristische Stufen der Kristallschädigung. Beginnend bei kleinen Fluenzen nimmt in einem ersten Bereich die Gitterschädigung linear mit der Fluenz zu, was auf die Erzeugung von Primärdefekten zurückzuführen ist. Darauf folgt ein Plateaubereich bei einer Gitterschädigung von etwa 1\%, was auf eine Überlappung der Defektkaskaden einzelner Ionen und ein damit verbundenes dynamisches Ausheilen von Defekten während der Implantation zurückzuführen ist. Bei steigender Fluenz wächst die Gitterschädigung überproportional an, was auf die oben beschriebenen, stabilen Defektkomplexe hinweist. Der vierte Bereich bei den höchsten Fluenzen ist wiederum ein Plateau und kann in Zinkoxid nur bei der Implantation mit Argon und bei sehr hohen Fluenzen $\left(10^{16}-10^{17} \mathrm{~cm}^{-2}\right)$ beobachtet werden. Üblicherweise wird dieser Bereich der vollständigen Amorphisierung eines Materials zugeordnet; bei Zinkoxid hingegen ist die Schädigung im Bereich von $50 \%$. In keinem der in der Literatur beschriebenen Experimente wurde eine Amorphisierung von Zinkoxid beobachtet, was in Übereinstimmung mit dem Kriterium der Bindungsart ist [Nag75]. Bei welchen Fluenzen welcher Bereich einsetzt hängt von der Masse und Energie der Ionen ab. So reicht der erste Plateaubereich für Erbium bis zu einer Fluenz von $\approx 10^{13} \mathrm{~cm}^{-2}$, während er für Stickstoff bis $\approx 10^{15} \mathrm{~cm}^{-2}$ reicht. Dies ist ein weiteres Argument dafür, dass die Stickstoff-Implantationsexperimente in Abschnitt 3.7 bei höherer Dosis keine Verstärkung der DAP zur Folge hatte. Die hier beschriebenen Experimente mit Seltenen Erden und hoher Fluenz sind deutlich im dritten Bereich, der vermehrten Entstehung thermisch stabiler Defektkomplexe, anzusiedeln. Im Übrigen zeigen weitere Experimente, dass der erste Bereich bei einer Implantati- 
on bei Raumtemperatur nicht auftritt [Lor05]. Primäre Defekte werden in Zinkoxid zwischen $80 \mathrm{~K}$ und $130 \mathrm{~K}$ mobil.

\subsection{Strukturelle Veränderungen von Galliumarsenid- Nanodrähten durch lonenbestrahlung}

Das Material Galliumarsenid mit einer Bindungsionizität von 0,31 amorphisiert im Gegensatz zu Zinkoxid bereits bei der Bestrahlung mit niedrigen Ionenfluenzen [Nag75, Wes92]. Im Folgenden werden Implantationsexperimente an Nanodrähten vorgestellt, die in der Duisburger Kollaboration hergestellt wurden. Zunächst werden die strukturellen Eigenschaften der hergestellten Nanodrähte dargestellt und die Implantationsparameter beschrieben. Im zweiten Unterabschnitt werden die strukturellen Veränderungen der Nanodrähte nach der Implantation mit Zinkionen diskutiert.

\subsubsection{Präparation und strukturelle Eigenschaften der Proben}

Die verwendeten Nanodrähten wurden in einem MOCVD-Prozess hergestellt, der in Abschnitt 2.1.4 beschrieben ist. Als Wachstumssubstrat wurden Galliumarsenid$\langle 100\rangle$-Kristalle verwendet. Die Wachstumszeit betrug 10 min bei einer Temperatur von $450{ }^{\circ} \mathrm{C}$, einem V/III-Gasangebot von etwa 5 und einem Gasfluss von 3,4 l/min bei 50 mbar. Weitere Details zum Wachstum sind in Ref. [Sti08b] zu finden.

Abbildung 4.11 b) zeigt die SEM-Aufnahme einer Galliumarsenid-Nanodrahtprobe nach dem Wachstum. Im SEM-Bild lassen sich charakteristische Winkel zwischen Nanodraht und Substrat beobachten, was auf eine erfolgreiche Epitaxie hinweist. Die Aufnahme wurde unter einem flachen Winkel aufgenommen, wobei die Probe im SEM so orientiert wurde, dass eine der auftretenden Wachstumsrichtungen eine senkrechte Projektion in der Bildebene hat. Der Durchmesser der Nanodrähte ist monodispers und beträgt $\approx 150 \mathrm{~nm}$, wobei dieser zum unteren Ende der Drähte leicht zunimmt. Dies wird, wie in Abschnitt 2.1.2 beschrieben, auf eine direkte Deposition an den Seitenflächen zurückgeführt. Alle Drähte haben eine Länge von $\approx 13 \mu \mathrm{m}$.

Untersuchungen im TEM, wie sie in Abbildung 4.11 a) dargestellt sind, zeigen in 

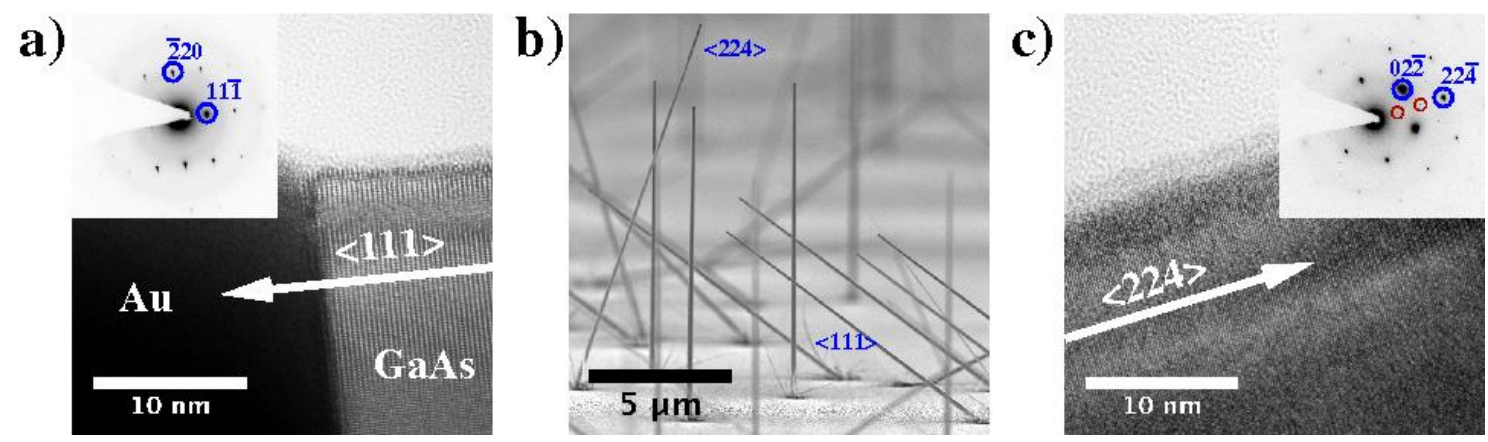

Abbildung 4.11: Elektronenmikroskopische Aufnahmen von Galliumarsenid-Nanodrähten. a) Hochauflösende TEM-Aufnahme eines Galliumarsenid-Nanodrahtes mit einem Goldkügelchen an dessen Ende. Die Wachstumsrichtung kann aus dem Beugungsbild zu einer $\langle 111\rangle$-Richtung bestimmt werden. b) SEM-Aufnahmen der gewachsenen Nanodrähte unter flachem Winkel. Es treten charakteristische Winkel auf, was auf eine erfolgreiche Epitaxie hinweist. c) Hochauflösende TEMAufnahme eines Galliumarsenid-Nanodrahtes, dessen Wachstumsrichtung aus dem Beugungsbild im Einsatz zu $\langle 112\rangle$ bestimmt werden kann.

Übereinstimmung mit der Literatur [Wac06, Per04], dass bei einem sehr großen Anteil der Nanodrähte die Wachstumsrichtung einer 〈111〉-Richtung des Kristalls entspricht. Am Ende aller Nanodrähte können im TEM Goldpartikel beobachtet werden, wie es entsprechend dem Wachstumsprozess zu erwarten war. Es ergeben sich lediglich sehr geringe Einschnürung zwischen Goldpartikel und Nanodraht. Dies weist darauf hin, dass das Ende des Wachstumsprozesses wohl definiert ist. Weiterhin konnten in TEM-Untersuchungen sehr vereinzelt Nanodrähte mit einer zweiten Wachstumsrichtung beobachtet werden (vgl. Abbildung $4.11 \mathrm{c}$ )). Die Wachstumsrichtung der Nanodrähte konnte entsprechend dem Beugungsbild einer $\langle 112\rangle$ Richtung des Kristalls zugeordnet werden. Die Beugungsbilder in Abbildung 4.11 a) und c) wurden an einzelnen Nanodrähten aufgenommen und zeigen, dass diese Einkristalle sind. Die unterschiedlichen Winkel zwischen Substrat und Nanodrähten, die in Abbildung 4.11 b) beobachtet werden, lassen sich folglich auf die vier $\langle 111\rangle$ Richtungen und zu einem geringen Anteil auch auf die zwölf $\langle 112\rangle$ Richtungen zurückführen, die die (100)-Ebene des Wachstumssubstrats durchstoßen.

In den Beugungsbildern der Wachstumsrichtung $\langle 112\rangle$ in Abbildung $4.11 \mathrm{c}$ ) treten relativ schwache Reflexe bei $\frac{1}{3}\{224\}$ auf, die zunächst nicht erwartet werden. Eine nähere Betrachtung zeigt, dass aufgrund der Stapelfolge von $\{112\}$-Ebenen nur jede dritte Einheitszelle an einer $\{111\}$-Ebene abgeschlossen wird. Wenn nun $\{111\}$ - 
a)

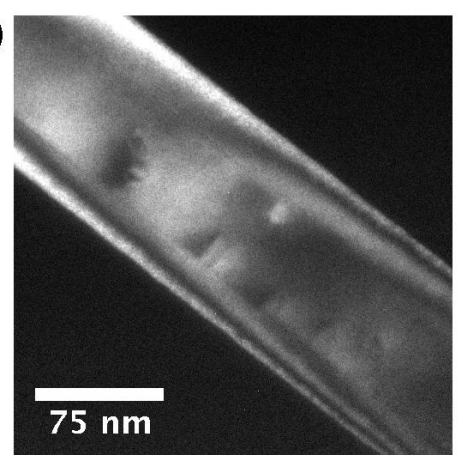

b)

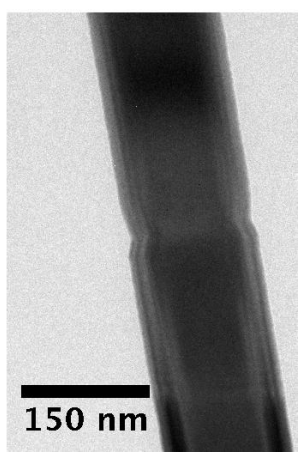

Abbildung 4.12: Defekte in Galliumarsenid-Nanodrähten. a) Die Dunkelfeldaufnahme zeigt lokale Defekte am Fuße eines Galliumarsenid-Nanodrahtes. b) In der Hellfeldaufnahme eines Galliumarsenid-Nanodrahtes ist eine leichte Einschnürung zu erkennen.

Ebenen Seitenfläche der Nanodrähte sind, ist dort die Braggbedingung für $\frac{1}{3}\{224\}$ Reflexe erfüllt. Dies führt zu dem Schluss, dass die Seitenflächen der Nanodrähte $\{111\}$-Ebenen sind, und dass diese über größere Bereiche atomar glatt sind, was ein weiteres Indiz für eine hohe Kristallqualität und ein wohldefiniertes Wachstum der Galliumarsenid-Nanodrähte ist.

In der Literatur werden häufig Kristalldefekte in Form von Stapelfehlern [Per04] und Zwillingsgrenzen [Joh06] an Galliumarsenid-Nanodrähten beobachtet. An den hier untersuchten Nanodrähten konnten hingegen nur sehr vereinzelt Kristalldefekte beobachtet werden. Diese traten in Form von einzelnen Stapelfehlern am Ende der Drähte auf. Neben den Kristalldefekten konnten bei niedrigen Vergrößerungen vereinzelt lokale Defekte am unteren Ende der Nanodrähte beobachtet werden, wie sie in einer Dunkelfeldaufnahme in Abbildung 4.12 a) gezeigt sind. Außerdem wurden gelegentlich leichte Einschnürungen an den Nanodrähten beobachtet. Eine entsprechende Aufnahme ist in Abbildung 4.12 b) gezeigt. Von diesen Einschnürungen konnten bis zu drei entlang eines Nanodrahtes beobachtet werden.

Die hergestellten Nanodrähte wurden im Folgenden bei Raumtemperatur mit Zink implantiert. Dabei wurde ein Teil der Probe abgedeckt, was einen direkten Vergleich der Nanodrähte nach der Implantation ermöglicht. Um eine Kanalisierung der Ionen im Kristallgitter zu verhindern, wurde die Implantation unter einem Winkel von $\approx 6^{\circ}$ zur Oberflächennormale des Substrats durchgeführt. Die Implantation erfolgte mit verschiedenen Ionenenergien zwischen 20 und $450 \mathrm{keV}$. Die resultierende 
a)

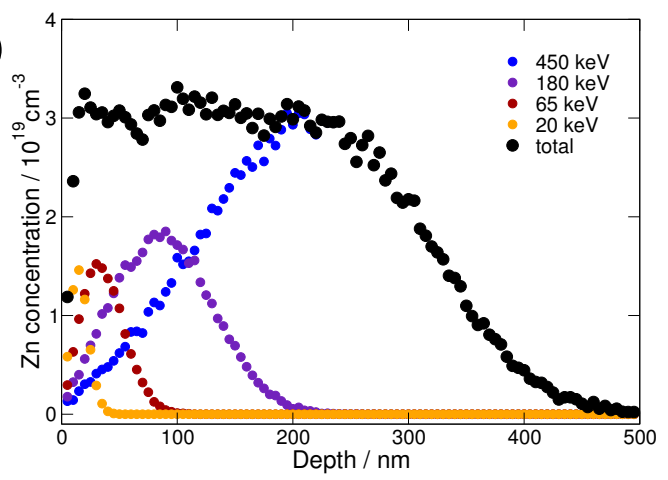

b)

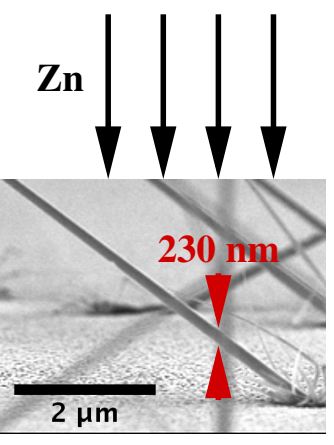

Abbildung 4.13: Implantationsparameter für die Zinkimplantation in GalliumarsenidNanodrähte. a) Das Implantationsprofil, wie es mit TRIM für die gewählte Ionenfluenz von $10^{15} \mathrm{~cm}^{-2}$ berechnet wurde. Die Überlagerung der Tiefenverteilung von vier Ionenenergien ergibt ein homogenes Konzentrationsprofil von $3 \cdot 10^{19} \mathrm{~cm}^{-3}$ über eine Tiefe $250 \mathrm{~nm}$. Dies entspricht der projizierten Dicke der gewachsenen Galliumarsenid-Nanodrähte. b) SEM-Aufnahme am unteren Ende eines Galliumarsenid-Nanodrahtes. Die projizierte Dicke beträgt etwa $230 \mathrm{~nm}$.

Zinkkonzentration ist über eine Tiefe von $250 \mathrm{~nm}$ konstant, was in etwa dem projizierten Durchmesser der Nanodrähte entspricht (vgl. Abbildung 4.13 b)). So wurde der Draht über seine gesamte Dicke homogen implantiert. Die verwendete Ionenfluenz betrug $10^{15} \mathrm{~cm}^{-2}$, was zu einer Zinkkonzentration von $3 \cdot 10^{19} \mathrm{~cm}^{-3}$ führet. Das mit TRIM berechnete Implantationprofil ist in Abbildung 4.13 a) dargestellt.

\subsubsection{Amorphisierung durch lonenimplantation und Ausrichtung der Nanodrähte}

Abbildung 4.14 a) zeigt eine SEM-Aufnahme der Galliumarsenid-Nanodrähte direkt nach der Implantation. Die Aufnahme erfolgte wiederum unter einem flachen Winkel, wobei die Probe so orientiert wurde, dass eine der Wachstumsrichtungen eine senkrechte Projektion in der Bildebene hat. Das SEM-Bild zeigt den Übergangsbereich zwischen dem Teil der Probe, der während der Implantation abgedeckt war (oberer Bildbereich) und dem Teil, der implantiert wurde. Während die Nanodrähte im nicht implantierten Bereich nach wie vor gerade sind und charakteristische Winkel mit der Substratoberfläche einschließen, sind die Nanodrähte im implantierten Bereich der Probe verbogen. Eine solche Verbiegung konnte in einem Referenzexperiment, in dem die Nanodrähte mit einer um eine Größenordnung 

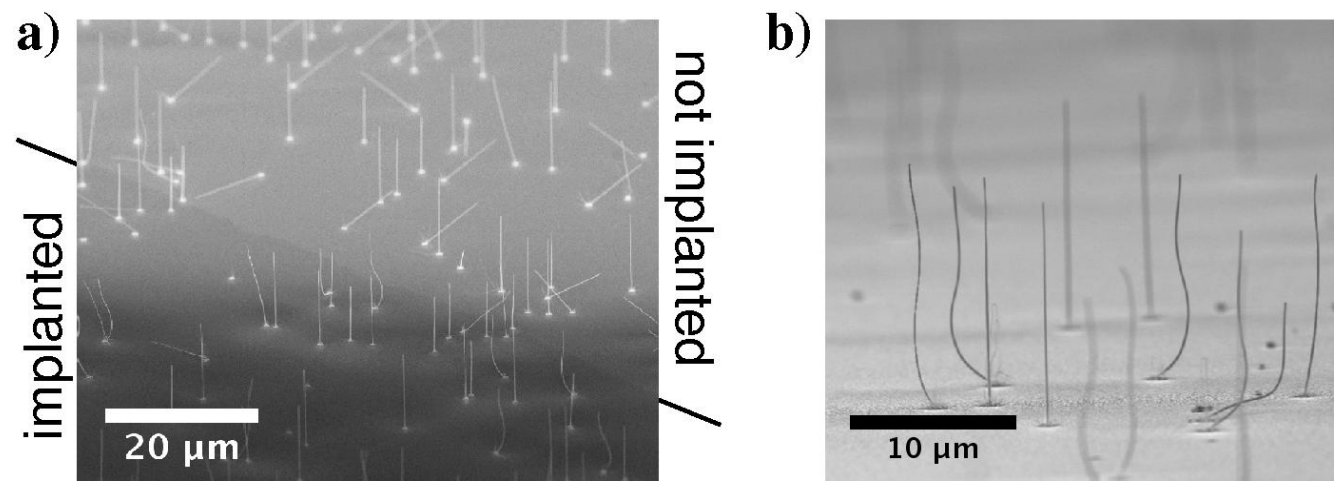

Abbildung 4.14: SEM-Aufnahme der mit Zink implantierten Galliumarsenid-Nanodrähte. a) Die Aufnahme zeigt einen Übergangsbereich zwischen implantiertem (unten) und nicht implantiertem Bereich (oben) der Probe. b) Die Galliumarsenid-Nanodrähte sind nach der Implantation in Richtung des einfallenden Ionenstrahls verbogen.

geringeren Zink-Ionenfluenz bestrahlt wurden, nicht beobachtet werden.

Abbildung 4.14 b) zeigt den implantierten Bereich der Probe mit einer höheren Vergrößerung. Die Verbiegung erfolgte bei allen Nanodrähten nach oben in Richtung des Ionenstrahls. Die Drähte, die in den Aufnahmen gerade wirken, sind in die Bildeben hinein verbogen. Dies wird in der SEM-Aufnahme durch die Tiefenschärfe deutlich, kann im SEM aber auch unter einem veränderten Betrachtungswinkel direkt beobachtet werden. Diese SEM-Aufnahme zeigt, dass die Verbiegung der Nanodrähte nahezu ausschließlich in der Ebene stattfindet, die von der Einfallsrichtung des Ionenstrahls (in etwa senkrecht zur Substratoberfläche) und der Wachstumsrichtung aufgespannt wird.

Um einen Aufschluss über die Ausrichtung der Nanodrähte durch die Ionenimplantation zu bekommen, wurden TEM-Untersuchungen an den implantierten Nanodrähten durchgeführt.

Dazu wurden zunächst Nanodrähte auf ein TEM-Netz übertragen. Abbildung 4.15 d) zeigt eine Übersichtsaufnahme eines typischen Nanodrahtes auf dem TEM-Netz. Der Draht hat eine Länge von $\approx 11 \mu \mathrm{m}$, was in etwa der Länge der Drähte vor der Implantation entspricht und ist von links nach rechts leicht verjüngt, was mit den Beobachtungen in SEM-Untersuchungen an den nicht implantierten Nanodrähten übereinstimmt. Desweiteren kann eine deutliche Krümmung des Drahtes beobachtet werden, ähnlich wie sie sich in den SEM-Bildern in Abbildung 4.14 

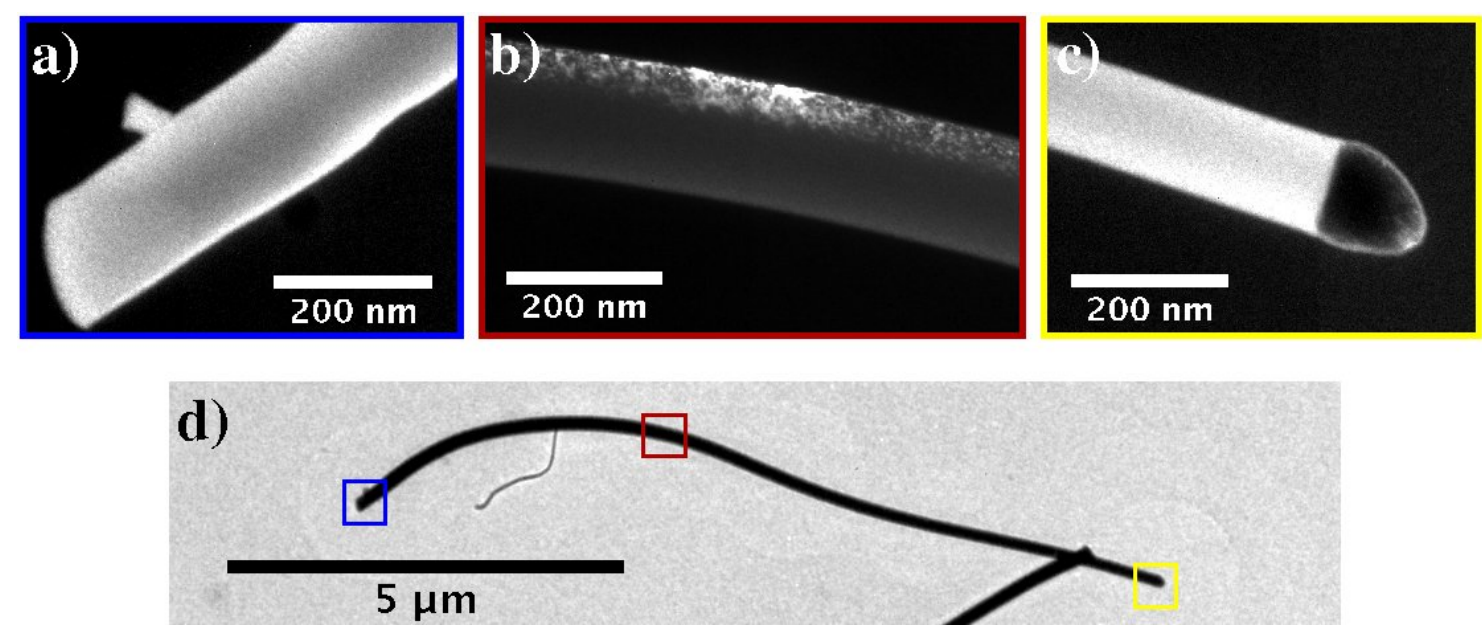

Abbildung 4.15: TEM-Dunkelfeldaufnahmen an unterschiedlichen Positionen entlang eines mit Zink implantierten Nanodrahtes. Der Draht ist am Anfang und Ende vollständig amorph, während in der Mitte, im Bereich der größten Krümmung, eine gewisse Restkristallinität vorhanden ist. Im unteren Bildteil ist eine Übersichts-Hellfeldaufnahmen gezeigt, an der die Positionen der Aufnahme farbig markiert sind.

darstellt. Dunkelfeldaufnahmen an unterschiedlichen Positionen entlang des Drahtes sind in Abbildung 4.15 a) bis c) dargestellt. An beiden Enden der Drähte kann kein Kontrast in den Nanodrähten beobachtet werden, was ein Indiz dafür ist, dass die Drähte hier vollständig amorphisiert sind. Im mittleren Teil dieses Nanodrahtes konnte eine gewisse Restkristallinität beobachtet werden. Untersuchungen an mehreren Nanodrähten zeigten, dass diese Restkristallinität vorwiegend dort, wo die Krümmung des Nanodrahtes am größten ist, auf der konvexen Seite auftritt. Die konvexe Seite der Nanodrähte war entsprechend der SEM-Bilder vor der Implantation dem Wachstumssubstrat zugewandt. Bezüglich der eintreffenden Ionen war dies die Rückseite der Nanodrähte. Es ergibt sich also eine ähnliche Situation, wie sie bereits bei der Implantation der Seltenen Erden in Zinkoxid beobachtet wurde (vgl. Abbildung 4.10 a)). Unterstützt wird diese Vermutung dadurch, dass bei dickeren Nanodrähten entlang der gesamten Länge eine Restkristallinität auftritt (vgl. Abbildung 4.17 b)).

Abbildung 4.16 zeigt hochauflösende TEM-Aufnahmen an unterschiedlichen Positionen entlang des Drahtdurchmessers in dem Bereich, in dem eine Kontraständerung in den Dunkelfeldaufnahmen zu beobachten ist. In dem Bereich 

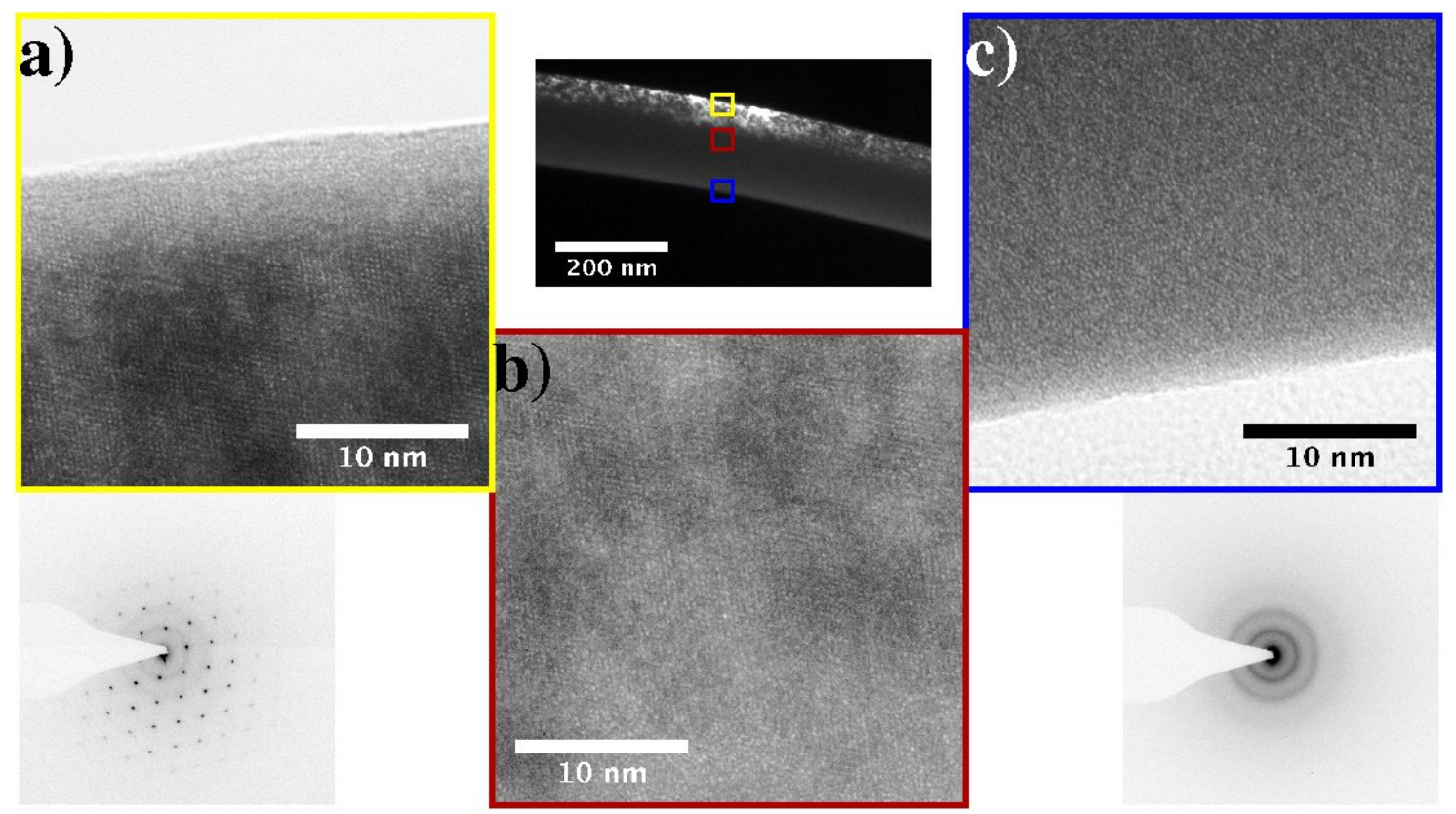

Abbildung 4.16: Hochauflösende TEM-Untersuchung und Elektronenbeugung an verschiedenen Positionen entlang des Drahtdurchmessers in dem Bereich, wo ein starker Kontrast in den Dunkelfeldaufnahmen beobachtet wird. a) Im Bereich der hellen Kontraste kann eine Kristallinität beobachtet werden, was durch Elektronenbeugung bestätigt wird. b) Übergangsbereich zwischen kristallinem und amorphem Bereich. Im oberen Teil des Bildes sind Gitterebenen zu erkennen, der untere Teil wirkt amorph. c) In dem Bereich ohne Kontrast in der Dunkelfeldaufnahme kann eine amorphe Struktur beobachtet werden, was durch lokale Elektronenbeugung bestätigt wird. In der Dunkelfeldaufnahme sind die Positionen der hochauflösenden Aufnahmen markiert.

des Nanodrahtes, in dem helle Kontraste zu beobachten sind, kann in dem hochauflösenden TEM-Bild in der Tat eine Kristallstruktur beobachtet werden (vgl. Abbildung 4.16 a)). Dies kann durch eine lokale Elektronenbeugung bestätigt werden. Abbildung 4.16 b) zeigt den Übergang zwischen einem kristallinen und einem amorphen Bereich. Im oberen Bildteil ist das Kristallgitter noch zu erkennen, während der untere Bildteil bereits eine amorphe Struktur zeigt. Eine Aufnahme der unteren Seite des Nanodrahtes, der in der Dunkelfeldaufnahme nahezu keinen Kontrast zeigt, ist in Abbildung 4.16 c) dargestellt. Hier ist in der Tat lediglich eine amorphe Struktur zu erkennen, was auch eine lokale Elektronenbeugung in diesem Bereich bestätigt.

Dass sich der Nanodraht gerade zur amorphen Seite hin krümmt, kann aufgrund der 

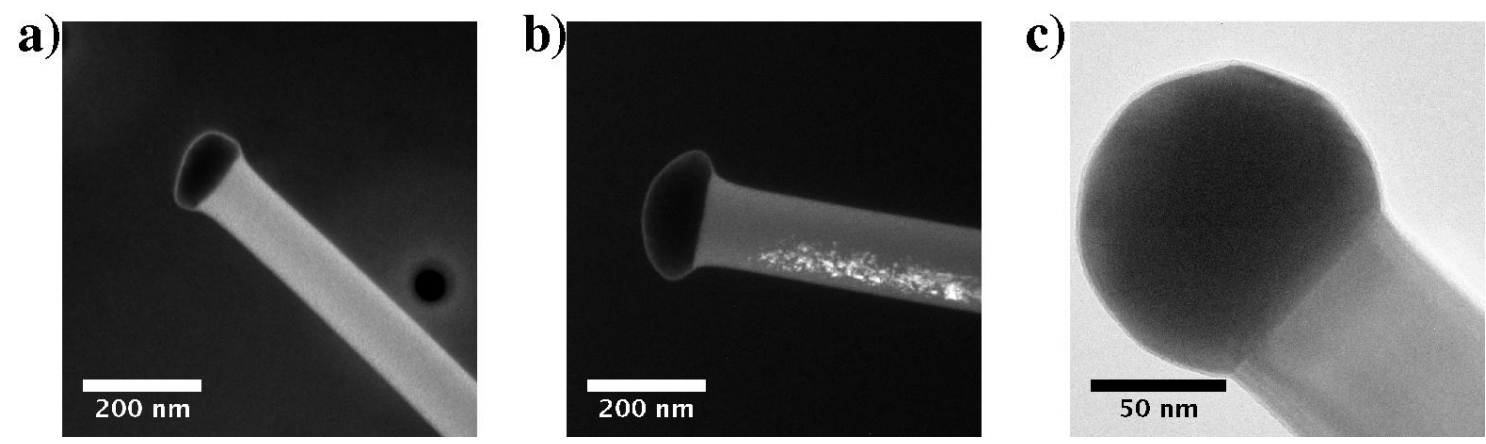

Abbildung 4.17: TEM-Aufnahmen von den Enden der Nanodrähte mit Goldpartikel. a) und b) In den TEM-Dunkelfeldaufnahmen der implantierten Nanodrähte ist eine Abflachung des Goldpartikels zu erkennen. Die Drähte selbst sind zum großen Teil amorph, wobei an dem dickeren Draht eine Restkristallinität beobachtet werden kann. c) Das Ende eines Galliumarsenid-Nanodrahtes vor der Implantation zum Vergleich.

TEM-Untersuchungen allerdings nicht verstanden werden, denn die Amorphisierung von Galliumarsenid ist mit einer Vergrößerung des spezifischen Volumen um 4\% verbunden [Rid98], wonach gerade eine Krümmung in die entgegengesetzte Richtung zu erwarten wäre.

Neben der Veränderung der Kristallinität und der Verkrümmung kann eine Veränderung des Goldpartikels beobachtet werden. Während dieser vor der Implantation bei allen Drähten eine runde Form hatte (vgl. Abbildung 4.17 c)), ist der Goldpartikel in Abbildung 4.15 c) annähernd elliptisch. Auch an anderen Nanodrähten konnte eine Veränderung des Goldpartikels beobachtet werden, wobei die Formen der Goldpartikel nach der Implantation deutlich verschieden sind. In Abbildung 4.15 a) und b) sind zwei weitere Dunkelfeldaufnahmen von Goldpartikeln anderer Nanodrähte dargestellt. An diesen wirkt der Goldpartikel deutlich abgeflacht. An den beiden hier dargestellten Nanodrähten hat sich eine leichte Auswölbung des Halbleitermaterials zwischen Goldpartikel und Nanodraht ergeben. Dies sollte auf einen unterschiedlichen Materialabtrag durch einen Sputterprozess in der Nähe des Goldpartikels zurückzuführen sein. Insgesamt wird im Vergleich zu dem in Abschnitt 4.1.2 beschriebenen Experiment eine deutlich geringere strukturelle Veränderung durch einen Sputterprozess erwartet. Zwar werden hier ähnlich Fluenzen verwendet, der Sputterkoeffizient ist allerdings deutlich geringer $(\approx 5 \mathrm{At}$./Ion bei senkrechtem Einfall). Dies resultiert bei der verwendeten Fluenz in einem Ma- 
terialabtrag von etwa 5 Monolagen $(2 \mathrm{~nm}$ ), vorausgesetzt der Nanodraht hat einen Großteil der Fluenz inkorporiert und sich nicht bereits zu Beginn der Implantation in Richtung des Ionenstrahls ausgerichtet. Hiervon kann jedoch ausgegangen werden, denn zum einen zeigte ein Referenzexperiment, dass sich die Drähte bei niedrigerer Fluenz nicht verbiegen, zum anderen sind die Nanodrähte vollständig amorphisiert, was ein Hinweis auf eine hohe Ionendosis ist.

\subsubsection{Rekristallisation durch Temperaturbehandlung}

Die implantierten und teilweise amorphisierten Proben wurden einer Temperaturbehandlung unterzogen. Dies ist bei Galliumarsenid ein relativ kritischer Prozess, da das Arsen bereits bei $500{ }^{\circ} \mathrm{C}$ flüchtig wird [Wes92]. Zwar wird eine Rekristallisation amorpher Schichten auf einem kristallinen Substrat bereits bei Temperaturen zwischen $150{ }^{\circ} \mathrm{C}$ und $400{ }^{\circ} \mathrm{C}$ beobachtet, für die angestrebte elektrische Aktivierung des Zinks sind allerdings Temperaturen über $750^{\circ} \mathrm{C}$ notwendig [Wes92, Kul80]. Um eine Veränderung der Stochiometrie der amorphen Galliumarsenid-Nanodrähte durch die Temperaturbehandlung zu vermeiden, wurde die Probe im MOVPE-Reaktor unter TBAs-Gegendruck getempert. Die Temperaturbehandlung erfolgte bei $800{ }^{\circ} \mathrm{C}$ für 30 min. Anschließend wurden wiederum TEM-Untersuchungen an den Nanodrähten durchgeführt.

Abbildung 4.18 a) zeigt eine invertierte Dunkelfeldaufnahme eines implantierten und getemperten Nanodrahtes. Es zeigen sich deutliche Kontraste in der Aufnahme, was auf eine Rekristallisation der Nanodrähte hinweist. Hochaufösende TEMAufnahmen zeigen, dass die Drähte lokal vollständig rekristallisiert sind (vgl. Abbildung 4.18 b)). Eine Elektronenbeugung an einzelnen Nanodrähten zeigt weiterhin, dass diese wiederum Einkristalle sind (vgl. Abbildung $4.18 \mathrm{c}$ )). Allerdings bleiben in den Nanodrähten Versetzungen mit einer Dichte von $\approx 5 \cdot 10^{9} \mathrm{~cm}^{-2}$ zurück, was sich durch die dunklen Linien senkrecht zur Nanodrahtachse in Abbildung 4.18 a) äußert. Bei den Versetzungen handelt es sich um Reissverschlussversetzungen [Jon88], die vermutlich auf den Ausheilprozess zurückzuführen sind. Die Rekristallisation beginnt an den kristallinen Bereichen der Nanodrähte, die den Implantationsprozess überstanden haben. In dem Bereich, an dem zwei Kristallisationsfronten aufeinander treffen, ist ein defektfreier Übergang nicht sehr wahrscheinlich. Auch bei epitakti- 


\title{
a)
}

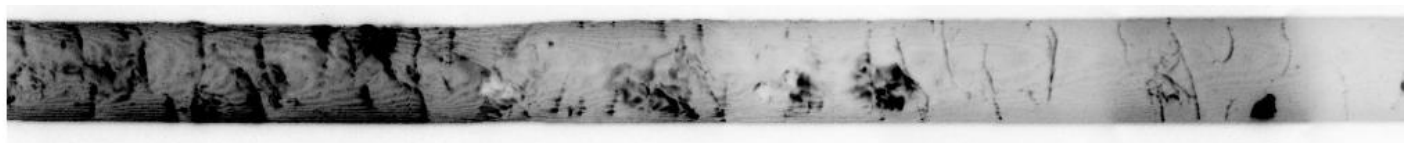

$500 \mathrm{~nm}$

b)

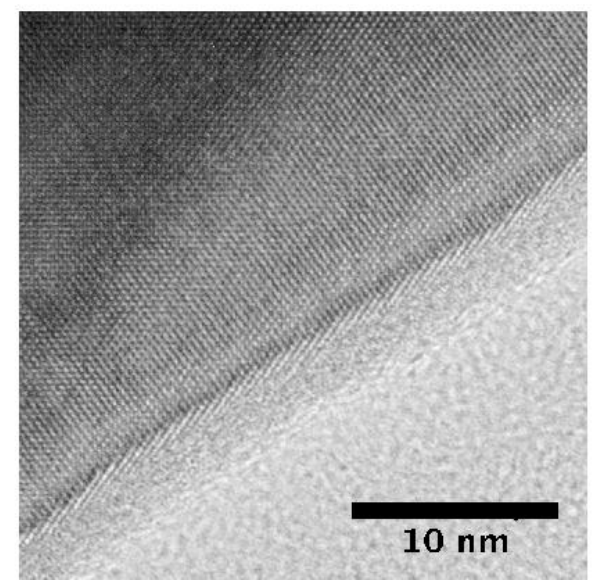

c)

\begin{abstract}
Abbildung 4.18: TEM-Untersuchungen an zinkimplantierten Galliumarsenid-Nanodrähten nach einer Temperaturbehandlung bei $800{ }^{\circ} \mathrm{C}$. a) In der invertierten Dunkelfeldaufnahme sind insbesondere Reissverschlussversetzungen zu erkennen. b) Die hochaufösende TEM-Aufnahme zeigt die vollständige Wiederherstellung des Kristallgitters. c) Eine Elektronenbeugung an einem Nanodraht zeigt, dass dieser wieder ein Einkristall ist.
\end{abstract}

scher Rekristallisation amorpher Galliumarsenid-Schichten wurden nach Temperaturbehandlungen zwischen $700{ }^{\circ} \mathrm{C}$ und $800{ }^{\circ} \mathrm{C}$ Versetzungen beobachtet [Kul80]. Obwohl die Ausgangsbedingungen im Vergleich zu der in der Literatur diskutierten epitaktischen Rekristallisation ungleich schlechter sind, konnte in dem beschriebenen Prozess eine vergleichbare Kristallinität erreicht werden.

\subsection{Zusammenfassung}

In diesem Kapitel wurden Untersuchungen zu den Auswirkungen einer Ionenbestrahlung auf die Morphologie und Kristallinität von Halbleiternanodrähten vorgestellt. Es wurden Experimente an den Materialsystemen Galliumarsenid und Zinkoxid vorgestellt, in denen stark unterschiedliche Implantationsbedingungen hinsichtlich der verwendeten Ionenmasse, -energie, -dosis und Geometrie verwendet wurden. Im ersten Abschnitt wurden Untersuchungen an Zinkoxid-Nanodrähten mit ver- 
gleichsweise geringem Durchmesser von $\approx 40 \mathrm{~nm}$ vorgestellt, die mit hohen Fluenzen schwerer Ionen (Seltenen Erden und Xenon) und geringer Ionenenergie bestrahlt wurden. Die implantierten Nanodrähte befanden sich dabei dispergiert auf einem Siliziumsubstrat. Mit zunehmender Ionenfluenz wurde an den Nanodrähten insbesondere eine charakteristische Veränderung der Morphologie und eine zunehmende Rauigkeit der Oberfläche beobachtet, was auf einen Abtrag von Oberflächenatomen durch einen Sputterprozess zurückgeführt wurde. Während die Nanodrähte vor der Implantation eine ansatzweise runde Querschnittsfläche hatten, konnte nach der Implantation eine ovale Form beobachtet werden. Diese Beobachtung konnte in einer ersten Näherung durch das Sputtermodell von Sigmund [Sig69] beschrieben werden. Im Detail erweist sich das betrachtete System für eine konsistente Beschreibung als sehr komplex. Da die Nanodrähte einen geringen Durchmesser haben unterscheiden sich die Winkel unter denen die Ionen auftreffen und damit auch die Sputterkoeffizienten lokal erheblich. Bereits durch dem Einschlag eines einzelnen Ions kann sich die Krümmung lokal erheblich verändern. Dies stellt für die weitere Bestrahlung eine vollständig neue Ausgangsbedingungen dar. Hinzu kommt die Readsorption von Atomen, die vom Siliziumsubstrat oder vom Nanodraht an anderer Stelle abgetragen wurden, der Einfluss der instantanen Rekristallisation, eine Oberflächendiffusion sowie die Änderung der Zusammensetzung des Nanodrahtes durch die Implantation. Damit unterliegt der Sputterprozess einer großen Dynamik.

Untersuchungen zur Kristallinität an implantierten und bei $700{ }^{\circ} \mathrm{C}$ getemperten Nanodrähten zeigen, dass die Kristallstruktur der Nanodrähte trotz der großen Ionendosis erhalten ist. Dies wurde insbesondere auf die hohe Bindungsionizität in Zinkoxid zurückgeführt. Dennoch zeigen hochauflösende TEM-Aufnahmen der implantierten Nanodrähte lokal starke Kontrastunterschiede, was auf eine lokale Störung des Kristallgitters durch Defektkomplexe hinweist. Ausscheidungen der implantierten Spezies konnten nicht beobachtet werden. Außerdem zeigte eine Elementanalyse, dass die Seltenen Erden in der erwünschten Konzentration in den Nanodrähten vorhanden sind.

Im zweiten Abschnitt wurden Untersuchungen an Galliumarsenid-Nanodrähten vorgestellt, die mit moderater Fluenz und hohen Energien mit Zinkionen implantiert wurden. Die implantierten Nanodrähte wurden in einem epitaktischen Wachstumsprozess hergestellt. TEM-Untersuchungen zeigten eine hervorragende Kristall- 
qualität der Nanodrähte. Außerdem konnten die $\langle 111\rangle$ und $\langle 112\rangle$-Richtungen als Wachstumsrichtungen der Nanodrähte bestimmt werden. Entsprechend schlossen die Nanodrähte mit dem Wachstumssubstrat charakteristische Winkel ein.

Nach der Implantation mit vergleichsweise hoher Ionenfluenz konnte interessanterweise eine Ausrichtung der Nanodrähte zum Ionenstrahl beobachtet werden, wobei die Verbiegung in der Ebene stattfand, die von dem Nanodraht und dem Ionenstrahl aufgespannt wurde. TEM-Untersuchungen an diesen Drähten zeigten, dass diese zu einem großen Teil amorphisiert sind, was aufgrund der geringen Bindungsionizität auch zu erwarten war. Lediglich im dem Bereich, in dem der Nanodraht die stärkste Krümmung aufweist, konnte eine Restkristallinität auf der dem Ionenstrahl abgewandten Seite festgestellt werden.

Nachdem die Nanodrähte einer Temperaturbehandlung unterzogen wurden, konnte eine vollständige Rekristallisation der Nanodrähte zu einem Einkristall beobachtet werden. Lediglich eine gewisse Anzahl von Versetzungen, die im Wesentlichen senkrecht zur Nanodrahtachse verlaufen, konnten beobachtet werden. 


\section{Kapitel 5}

\section{Leuchtzentren in Halbleiternanodrähten}

\subsection{Intra-3d-Lumineszenz im System in ZnS:Mn}

Das System ZnS:Mn gehört wohl zu den am Besten erforschten Systemen von Leuchtzentren in Halbleitermaterialien überhaupt und findet aufgrund einer Lumineszenz mit hoher Effizienz seit langer Zeit Anwendung in WechselstromDünnfilmelektrolumineszenz-Bauteilen (ACTFEL) [Mue00]. Ursprung dieser intensiven gelb-orangen Lumineszenz ist ein interner Übergang im $\mathrm{Mn}^{2+} 3 d^{5}$-System. An mangandotierten Zinksulfid-Kristallen wurde zum Teil beobachtet, dass die bandkantennahe Lumineszenz vollständig zugunsten dieser intra-3d-Lumineszenz ausgelöscht wird. Der Übergang selbst findet zwischen dem ersten angeregten Zustand ${ }^{4} T_{1}$ und dem Grundzustand ${ }^{6} A_{1}$ des $\mathrm{Mn}^{2+} 3 d^{5}$-Systems statt und hat eine Energie von $\approx 2,1 \mathrm{eV}$ (vgl. Abbildung $5.1 \mathrm{a})$ ). Der Spin des ersten angeregten Zustands beträgt 3/2, der des Grundzustand 5/2. Damit ist der Übergang entprechend der Auswahlregeln der Dipolnäherung für freie Manganionen spinverboten.

Ist das Manganion allerdings in einem Kristall eingebaut, so wird dieser Übergang unter anderem durch eine Wechselwirkung der Manganionen mit dem Kristallfeld und durch eine s, p-d Hybridisierung der Bindungen teilweise erlaubt [Bha94a]. Charakteristisch für teilweise erlaubte Übergänge sind lange Lebenszeiten - für das oben beschriebene System wurde bei sehr geringen Mangankonzentration eine intrinsische Lebenszeiten von 1,8 ms gemessen [Gum81]. Eine einfache exponentielle Entvölkerung des angeregten Zustands kann allerdings nur für ZnS:Mn-Kristalle 


\section{a)}

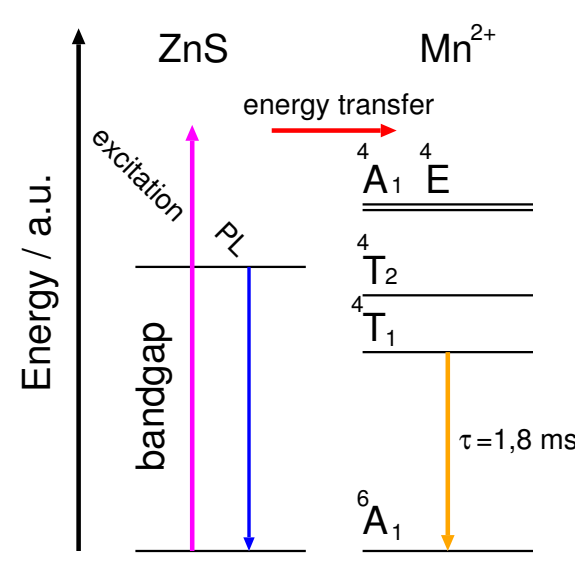

b)

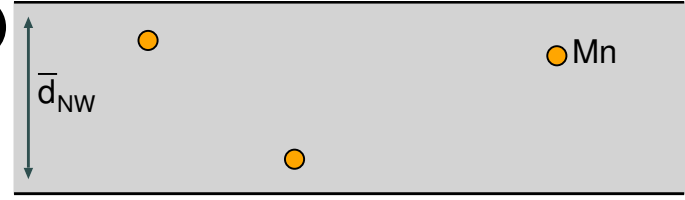

c)

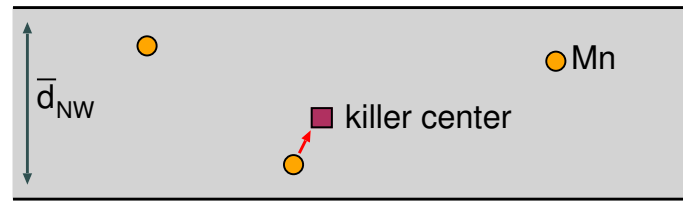

d)

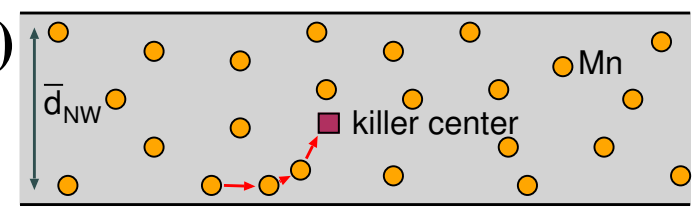

Abbildung 5.1: a) Schema der Energieniveaus von Wirtskristall und Mangansystem. b) - d) Abhängigkeit der Rekombinationsmechanismen von charakteristischen Abständen zwischen Manganionen und Zentren nichtstrahlender Rekombination (killer center). b) Isolierte Manganionen in einem defektfreien Kristall. c) Energietransfer zu Zentren nichtstrahlender Rekombination bei geringer Mangankonzentration und Defekten im Wirtskristall. d) Zusätzlicher Energietransfer zwischen Manganionen bei größerer Mangankonzentration.

mit ausgesprochen hoher Kristallqualität und geringer Mangankonzentration beobachtet werden (vgl. Abbildung 5.1 b)). In Material mit höherer Defektdichte wird eine kürzere und nicht exponentielle Lebenszeit der Manganlumineszenz beobachtet. Dies wird üblicherweise auf einen Energietransfer aus dem System angeregter Manganionen zu Zentren nichtstrahlender Rekombination (Killerzentren) zurückgeführt und lässt sich mit dem Modell des Förster-Energietransfers beschreiben [För49] (vgl. Abbildung 5.1 c)). Diese Zentren nichtstrahlender Rekombination treten üblicherweise im Zusammenhang mit Defekten im Kristall auf. Desweiteren zeigt die Lebenszeit eine starke Abhängigkeit von der Mangankonzentration im Kristall. Mit steigender Mangankonzentration nimmt die Lebenszeit typischerweise um mehr als zwei Größenordnungen ab [DV92]. Diese Beobachtung wird auf einen zusätzlichen Energietransfer zwischen den Manganionen zurückgeführt. Dadurch wird die Anregung des Systems effektiver zu den Killerzentren transportiert (vgl. Abbildung $5.1 \mathrm{~d})$ ).

Die typische Lebenszeit der Manganlumineszenz wird also durch zwei charakte- 
ristische Längen bestimmt: den mittleren Abstand zwischen den Manganionen im Wirtskristall und den mittleren Abstand zwischen Manganionen und Killerzentren. Beide Längen liegen im Bereich der typischen Ausdehnung von Nanostrukturen. Entsprechend ist es sinnvoll anzunehmen, dass die Strukturgröße des Wirtskristalls einen Einfluß auf die Lebenzeit der Manganlumineszenz hat.

Bisher wurde insbesondere die Lumineszenz sphärischer ZnS:Mn-Nanopatikel untersucht. Bei Nanopatikeln mit geringer Mangankonzentration wurde eine starke Reduktion der Lebenszeit um fünf Größenordnungen sowie eine sehr hohe Quanteneffizienz von 18\% beobachtet [Bha94a, Bha94b]. Mögliche Ursachen dieser Beobachtung sind Gegenstand einer langjährigen Diskussion. Als mögliche physikalische Ursachen der verkürzten Lebenszeit werden eine Vielzahl von Phänomenen angeführt. In einer ursprünglichen Arbeit wurde eine stärkere s, p-d Hybridisierung aufgrund der reduzierten Strukturgröße [Bha94a, Bha94b] als Ursache der verkürzten Lebenszeit und der hohen Quanteneffizienz angeführt [Bha96]. Neben der kurzen Lebenszeit ( $\approx 100 \mathrm{~ns}$ ), die auch in Ref. [Bha94a, Bha94b] beschrieben wurde, wird in Ref. [Bol98, Bol00, Smi00, Che05] eine Überlagerung mit der intrinsischen Lebenszeit $\approx 1,8 \mathrm{~ms}$ berichtet. Die kurze Lebenszeitkomponente wurde hier einer Lumineszenz zugeordnet, die im Zusammenhang mit Defekten im Zinksulfid bzw. mit der Oberfläche der Nanopartikel steht. Desweiteren wurde eine größenabhängige Elektron-Phononkopplung [Che01] sowie eine Mischung von d-Zuständen des Mangans mit Zuständen des Wirtskristalls durch Coulombwechselwirkung [Yan98] als physikalische Ursache der kurzen Lebenszeitkomponente der intra-3d-Lumineszenz des Mangans in Zinksulfid-Nanopatikeln genannt.

Im Folgenden wird systematisch der Einfluss der Morphologie der Wirtskristalle sowie der Mangankonzentration auf die Lebenszeit der Mangan intra-3d-Lumineszenz untersucht. Zur Beschreibung des zeitlichen Abklingens der Manganlumineszenz wird eine Abwandlung des Modells für einen Energietranfer zu Killerzentren nach Förster [För49] und Dexter [Dex53] für niedrige Dimensionen hergeleitet.

\subsubsection{Präparation der Proben}

Einkristalline Zinksulfidnanodrähte und -nanobänder mit unterschiedlichen lateralen Abmessungen wurden in einem VLS-Prozess hergestellt [Sti05, Sti06a]. 
a)

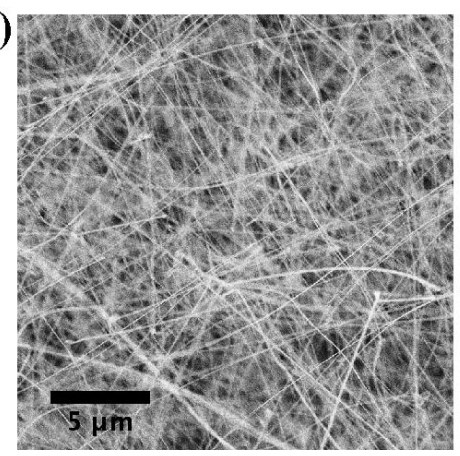

b)

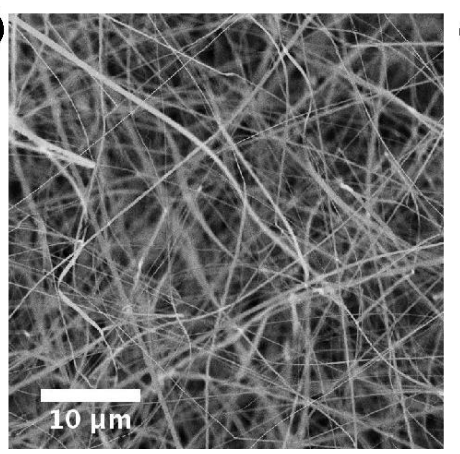

c)

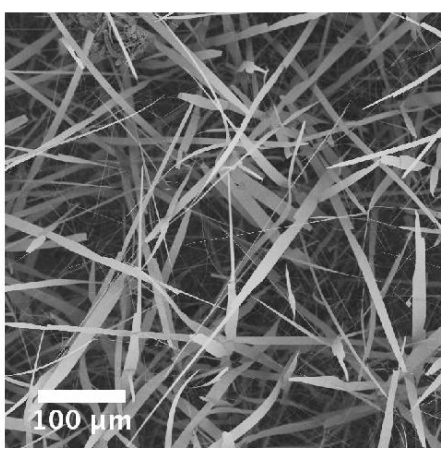

Abbildung 5.2: SEM-Aufnahmen von Zinksulfid-Nanodrähten mit verschiedenem mittleren Durchmesser von a) $100 \mathrm{~nm}$, b) $500 \mathrm{~nm}$ und c) $20 \mu \mathrm{m}$.

Abbildung 5.2 zeigt SEM-Aufnahmen der hergestellten Strukturen. Für identisch präparierte Wachstumssubstrate variiert der mittlere Durchmesser der synthetisierten Strukturen mit dem Abstand zum Ausgangsmaterial in dem Wachstumsprozess über mehr als zwei Größenordnungen zwischen $\approx 100 \mathrm{~nm}$ (Abbildung 5.2 a)) und $\approx 30 \mu \mathrm{m}$ (Abbildung $5.2 \mathrm{c}$ )). Bei den größeren Strukturen handelt es sich um bandartige Strukturen, deren Dicke $\approx 100 \mathrm{~nm}$ beträgt. Transmissionselektronenmikroskopie und Röntgendiffraktometrie zeigen, dass die Nanodrähte und -bänder überwiegend eine Zinkblende-Kristallstruktur haben und Einkristalle sind [Sti05, Sti06a].

Für die folgenden Untersuchungen wurden zwei Proben mit unterschiedlicher Morphologie ausgewählt: Nanodrähte mit einem Durchmesser von $\approx 100 \mathrm{~nm}$ (vgl. Abbildung 5.2 a)) und Nanobänder mit einer Breite von $\approx 30 \mu \mathrm{m}$ (vgl. Abbildung $5.2 \mathrm{c})$ ). In diese Strukturen wurde mittels Ionenimplantation Mangan eingebracht. Dazu wurden aus den Ausgangsproben zunächst jeweils mehrere gleichartige Proben hergestellt, indem die Drähte einer Ausgangsprobe, wie in Abschnitt 2.2.2 beschrieben, auf mehreren sauberen Substraten verteilt wurden. Die Proben wurden mit Manganionen fünf unterschiedlicher Energien zwischen $20 \mathrm{keV}$ und $450 \mathrm{keV}$ implantiert. Das Implantationsprofil resultiert in einer homogenen Mangankonzentration über eine Tiefe von $350 \mathrm{~nm}$; entsprechend ist von einer homogenen Mangankonzentration in den Nanostrukturen auszugehen. Die eingebrachte Mangankonzentration wurde über sechs Größenordnungen zwischen $4 \cdot 10^{-6}$ at.\% und 4 at.\% variiert, was in einem mittleren Manganionenabstand zwischen $\approx 2 \mathrm{~nm}$ und $\approx 200 \mathrm{~nm}$ resultiert. 
a)

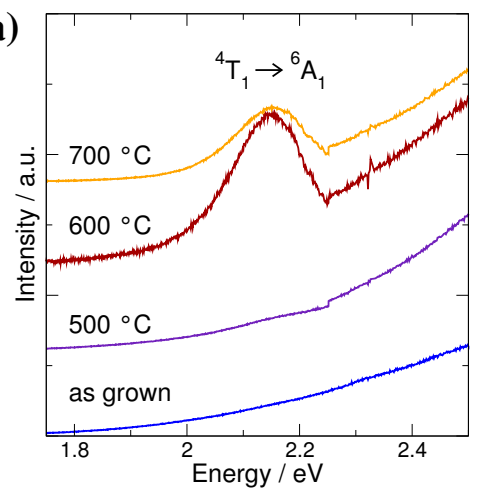

b)

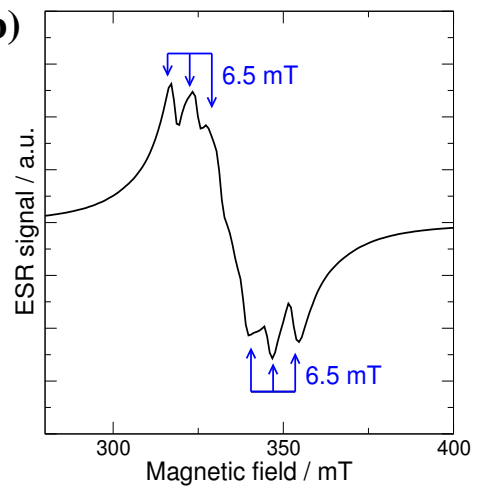

c)

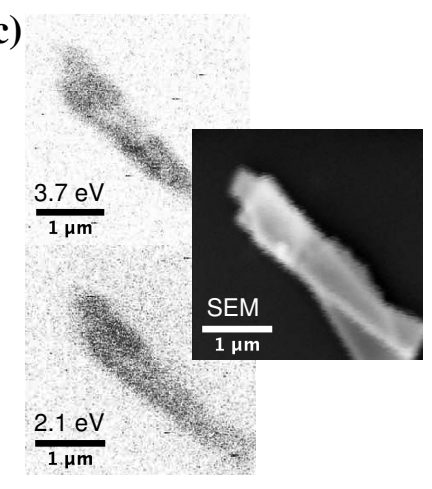

Abbildung 5.3: a) PL-Spektren einer Zinksulfidprobe nach dem Wachstum sowie nach der Implantation und anschließendem Tempern bei unterschiedlichen Temperaturen. b) Elektronspinresonanz-Messung an manganimplantierten und bei $600{ }^{\circ} \mathrm{C}$ getemperten ZinksulfidNanodrähten. c) Kathodolumineszenzuntersuchung im SEM an manganimplantierten und bei $600{ }^{\circ} \mathrm{C}$ getemperten Zinksulfid-Nanodrähten. Die Probentemperatur betrug $77 \mathrm{~K}$.

\subsubsection{Optische Aktivierung des Mangans}

Um eine optische Aktivierung des Mangans zu erlangen, müssen die implantierten Proben zunächst einer Temperaturbehandlung unterzogen werden. Zinksulfid verhält sich bei der Bestrahlung mit Ionen ähnlich wie Zinkoxid; eine Amorphisierung der Nanostrukturen tritt aufgrund der hohen Ionizität der Bindung nicht ein [Sti06b, Nag75]. Allerdings ist eine Temperaturbehandlung bei diesem Material kritischer als bei Zinkoxid, da der Schwefel in der Verbindung bereits bei niedriger Temperatur flüchtig wird [Geo77]. Um eine Oxidation des Zinksulfids zu vermeiden, wurde die Temperaturbehandlung im Vakuum $\left(10^{-6}\right.$ mbar) durchgeführt.

Um ideale Bedingungen für die optische Aktivierung der Manganlumineszenz nach der Ionenimplantation zu finden, wurden zunächst manganimplantierte Referenzproben für 30 min bei unterschiedlichen Temperaturen zwischen $500{ }^{\circ} \mathrm{C}$ und $700{ }^{\circ} \mathrm{C}$ getempert. Die Aktivierung der Manganlumineszenz wurde in Photolumineszenzmessungen überprüft. Abbildung 5.3 a) zeigt die entsprechenden Spektren zusammen mit dem Spektrum einer nichtimplantierten Probe in dem Spektralbereich, in dem die ${ }^{4} T_{1} \rightarrow{ }^{6} A_{1}$ Manganlumineszenz erwartet wird. Die Spektren wurden bei einer Temperatur von $15 \mathrm{~K}$ unter Anregung bei 4,66 eV aufgenommen und sind entlang der Ordinate verschoben aufgetragen. In den Spektren der unimplantierten Probe sowie der implantierten und bei $500{ }^{\circ} \mathrm{C}$ getemperten Probe sind in dem be- 
trachteten Spektralbereich lediglich die Flanken eines Defektbandes zu erkennen. Ab einer Ausheiltemperatur von $600{ }^{\circ} \mathrm{C}$ wird die charakteristische Manganlumineszenz bei $\approx 2,1 \mathrm{eV}$ beobachtet. Bei höheren Ausheiltemperaturen kann die Bande zwar weiter beobachtet werden, allerdings verliert sie deutlich an Intensität. Entsprechend wurde in den folgenden Experimenten zur optimalen Aktivierung eine Temperatur von $600{ }^{\circ} \mathrm{C}$ verwendet.

Um zu bestätigen, dass die Lumineszenz bei $2,1 \mathrm{eV}$ auf einen ${ }^{4} T_{1} \rightarrow{ }^{6} A_{1}$-Übergang des Mangans im Zinksulfid zurückzuführen ist und nicht auf selbigen Übergang in Siliziumoxid, dass sich potentiell während der Temperaturbehandlung an der Substratoberfläche gebildet haben könnte, wurden ortsaufgelöste Kathodolumineszenzmessungen (CL) im SEM durchgeführt. Die Untersuchungen wurden von Prof. A. Gustafsson/ Universität Lund, Schweden durchgeführt. Abbildung 5.3 c) zeigt drei SEM-Aufnahmen eines Zinksulfidnanobandes auf dem mitimplantierten Siliziumsubstrat. Es ist zum einen das typische Sekundärelektronenbild gezeigt ist, zum anderen die Lumineszenz. Die Lumineszenzbilder wurden in zwei unterschiedlichen spektralen Bereichen aufgenommen: im Bereich der Bandlücke von Zinksulfid bei $3,7 \mathrm{eV}$ und im Bereich des ${ }^{4} T_{1} \rightarrow{ }^{6} A_{1}$-Übergangs bei $2,1 \mathrm{eV}$. Die Probentemperatur betug dabei 70 K. Beide Lumineszenzbilder geben die Position des Nanobandes wieder, wie es das Sekundärelektronenbild zeigt. Dies bestätigt, dass die Lumineszenz tatsächlich von den manganimplantierten Nanodrähten stammt.

Um den Einbau der Manganionen in das Zinksulfid-Kristallgitter näher zu untersuchen wurden Elektronspinresonanz (ESR) Messungen an Referenzproben durchgeführt. Die Proben wurden äquivalent zu den Proben mit der höchsten Mangankonzentration von 4 at.\% präpariert, allerdings wurden keine dispergierten Drähte, sondern eine ursprüngliche Probe verwendet. Die Messungen wurden von Dr. H.A. Krug von Nidda in der Gruppe von Prof. Dr. A. Loidl/ Universität Augsburg durchgeführt.

Abbildung $5.3 \mathrm{~b}$ ) zeigt ein ESR-Spektrum der Probe, das bei einer Temperatur von $4 \mathrm{~K}$ bei $9,5 \mathrm{GHz}$ aufgenommen wurde. Das Spektrum zeigt die typische Form von $\mathrm{Mn}^{2+}$ auf Zink-Gitterplätzen in Zinksulfid [Ken95, Bor99, Kla04]. Es setzt sich aus einem Sextet scharfer Linien zusammen, deren Zentrum bei einem LandèFaktor von $g \approx 2$ liegt. Das Sextet ist auf erlaubte magnetische Dipolübergänge $\left(\Delta \mathrm{m}_{\mathrm{S}}= \pm 1, \Delta \mathrm{m}_{\mathrm{I}}=0\right)$ zwischen den durch Hyperfeinwechselwirkung aufge- 
spaltenen Zeemann-Niveaus des ${ }^{6} \mathrm{~S}_{5 / 2}$ Grundzustands der $\mathrm{Mn}^{2+} 3 \mathrm{~d}^{5}$-Elektronen zurückzuführen. Die Hyperfeinaufspaltung kommt durch die Wechselwirkung des $S=5 / 2$ Spins der ungepaarten $3 \mathrm{~d}^{5}$-Elektronen mit dem $I=5 / 2$ Spin der ${ }^{55} \mathrm{Mn}$ Atomkerne zustande [Kla04]. Die Hyperfeinaufspaltung zwischen benachbarten Niveaus beträgt für Mangan in Zinkblende bzw. Wurtzit etwa 6,5 mT [Ken95, Bor99], was in guter Übereinstimmung mit der im Spektrum beobachteten Aufspaltung ist (vgl. Abbildung 5.3 b)). Die relativ hohe Mangankonzentration in der Probe führt zu Dipol-Dipol- und Austauschwechselwirkung zwischen den Manganionen. Dadurch kommt eine breite Resonanz zustande, durch die das Sextet überlagert wird. Außerdem sind die Resonanzen des Sextets durch eine Überlagerung mit schwach auftretenden verbotenen Übergängen $\left(\Delta \mathrm{m}_{\mathrm{S}}= \pm 1, \Delta \mathrm{m}_{\mathrm{I}}= \pm 1\right)$ zwischen den Hyperfeinniveaus leicht verbreitert. Dass die verbotenen Übergänge nur schwach auftreten, ist ein Anzeichen für ein Kristallfeld mit tetraedrischer Symmetrie, wie es für ZinkGitterplätze in der Zinkblende der Fall ist [Kla04].

\subsubsection{Lebenszeitmessungen der Mangan-intra-3d-Lumineszenz}

Lebenszeitmessungen an der ${ }^{4} T_{1} \rightarrow{ }^{6} A_{1}$-intra-3d-Lumineszenz des Mangans wurden in Kollaboration mit der Gruppe Heimbrodt/ Universität Marburg durchgeführt. Zur Anregung der Lumineszenz wurde ein gepulster Nd:YAG-Laser (3,49 eV, 3,5 ns) verwendet. Die Spektren wurden mit einer lichtverstärkten CCD-Kamera mit einer Zeitaufösung von 1,5 ns aufgezeichnet. Die Proben befanden sich in einem HeliumBadkryostaten bei einer Temperatur von $10 \mathrm{~K}$.

Abbildung 5.4 a) zeigt Photolumineszenzspektren des spektralen Bereiches der ${ }^{4} T_{1} \rightarrow{ }^{6} A_{1}$-Lumineszenz zu unterschiedlichen Zeiten nach der Anregung. Die Spektren sind auf die maximale Intensität normiert und entlang der Ordinate verschoben aufgetragen. Die Integrationszeit der Spektren beträgt $1 \mu \mathrm{s}$ bis zu einer Zeit von $20 \mu$ s nach dem Anregungspuls, $10 \mu$ s bis zu einer Zeit von $200 \mu$ s nach dem Anregungspuls, $100 \mu \mathrm{s}$ bis zu einer Zeit von 2 ms nach dem Anregungspuls und $1 \mathrm{~ms}$ für die übrigen Spektren. Die Form und spektrale Position der beobachteten Lumineszenz ist in allen untersuchten Zeitbereichen identisch. Dies zeigt, dass in dem untersuchten Zeitfenstern ausschließlich die intra-3d-Lumineszenz das Spektrum dominiert. Eine Überlagerung mit dem Defektband, wie es das integrale Lu- 

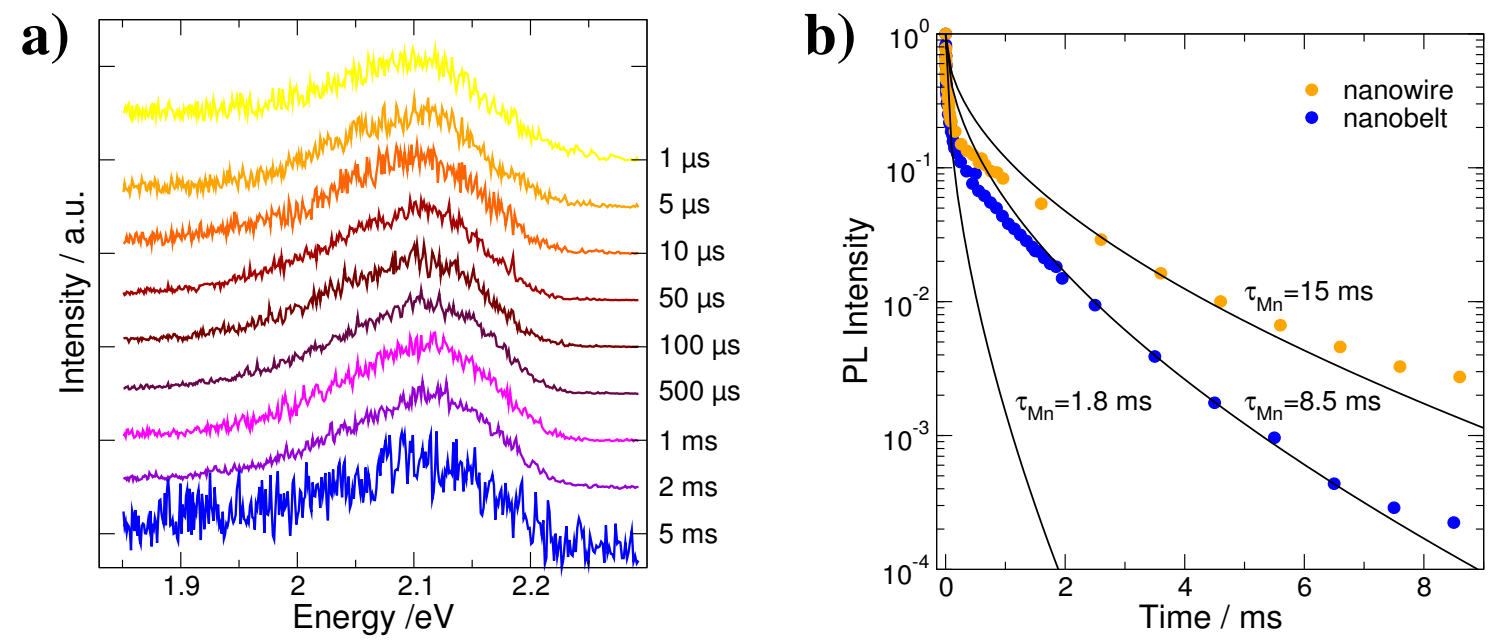

Abbildung 5.4: a) PL-Spektren von Zinksulfid-Nanobändern, die mit 4 at.\% Mangan implantiert wurden. Die Spektren wurden in unterschiedlichen Zeitfenstern nach der Anregung bei einer Probentemperatur von $10 \mathrm{~K}$ aufgezeichnet. b) Transienten der Manganlumineszenz in ZinksulfidNanodähten und Nanobändern mit einer Mangankonzentration von $4 \cdot 10^{-6}$ at.\%. Die durchgezogenen Linien wurden mit Gleichung 5.1 unter Annahme verschiedener intrinsischer Lebenszeiten der Manganlumineszenz $\tau_{M n}$ berechnet.

mineszenzspektrum in Abbildung 5.3 a) zeigt, ist nicht mehr gegeben, da diese wesentlich schneller abklingt. Die Transienten dieser Lumineszenz können mit dieser Methode über vier Größenordnungen gemessen werden, was essentiell für die folgende Auswertung ist.

Zunächst werden die Untersuchungen an den Proben mit der niedrigen Mangankonzentration von $4 \cdot 10^{-6}$ at.\% vorgestellt. Diese Konzentration entspricht einem mittleren Abstand der Manganionen von $\approx 200 \mathrm{~nm}$. Ein Energietransfer im Mangansystem wird in diesem Konzentrationsbereich nicht erwartet. Abbildung 5.4 b) zeigt die Transienten der Manganlumineszenz der Nanodrähte (orange Kreise, vgl. Abbildung 5.2 a)) und Nanobänder (blaue Kreise, vgl. Abbildung 5.2 c)). Beide Transienten zeigen ein deutlich nichtexponetielles Abklingen. Obgleich die Mangankonzentrationen in den Nanodrähten und Nanobändern identisch sind, unterscheiden sich die Transienten deutlich. Dies ist ein erstes Zeichen für eine Abhängigkeit von der Morphologie der Wirtskristalle. Im Bereich kurzer Zeiten nach der Anregung sind die Transienten zunächst durch ein schnelles Abklingen geprägt, dann folgt ein langsamer, nahezu exponentieller Bereich. Bei ZnS:Mn-Volumenkristallen mit 
vergleichbarem Mangangehalt lassen sich die nichtexponetiellen Transienten dieser Lumineszenz durch ein Energietransfer beschreiben, der auf dem Förstermodell beruht [För49]. Demnach kommt es durch eine Dipol-Dipol-Wechselwirkung zwischen angeregten Manganionen und Zentren nichtstrahlender Rekombination zu einem Energietransfer. Das zeitliche Verhalten der Luminesznz ist demnach gegeben durch

$$
\begin{aligned}
\left\langle\frac{I(t)}{I_{0}}\right\rangle & =\exp \left(-\frac{t}{\tau_{M n}}\right)\left[J\left(t, R_{g}\right)\right]^{N} \\
& \approx \exp \left(-\frac{t}{\tau_{M n}}\right) \exp \left(-\sqrt{\pi} \frac{N}{R_{g}^{3}} R_{0}^{3} \sqrt{\frac{t}{\tau_{M n}}}\right) \\
& =\exp \left(-\frac{t}{\tau_{M n}}\right) \exp \left(-\frac{4 \sqrt{\pi}^{3}}{3} n_{k i l l e r} R_{0}^{3} \sqrt{\frac{t}{\tau_{M n}}}\right)
\end{aligned}
$$

Dabei bezeichnet $\tau_{M n}$ die intrinsische Lebenszeit der Manganlumineszenz in Zinksulfid (1,8 ms), N die Anzahl der Zentren nichtstrahlender Rekombination und $R_{g}$ den Radius des als kugelförmig angenommenen Kristalls. Der Term $\frac{N}{R_{g}^{3}}$ lässt sich folglich auch als Dichte der Zentren nichtstrahlender Rekombination $\left(n_{\text {killer }}\right)$ ausdrücken. Die Größe $R_{0}$ bezeichnet eine kritische Länge, bei der die Rate für einen Energietransfer aus dem Mangansystem durch Dipol-Dipol-Wechselwirkung zu Killerzentren genauso groß ist wie die Rate für strahlende Rekombination. Diese Größe wurde in Messungen an ZnS:Mn-Nanopartikeln zu 4,85 nm bestimmt [Che07].

Die durchgezogenen Linien in Abbildung 5.4 b) wurden mit Gleichung 5.1 an die gemessenen Daten angepasst. Um mit Gleichung 5.1 den schnellen Beginn der Transienten beschreiben zu können, müssen relativ hohe Werte für $n_{\text {killer }}$ von $\approx 10^{19} \mathrm{~cm}^{-3}$ angenommen werden. Um eine Übereinstimmung bei größeren Zeiten nach der Anregung zu erlangen, müssen nicht realistische Werte für die intrinsische Lebenszeiten $\tau_{M n}$ gewählt werden. Doch auch mit diesen extremen Werte für die intrinsische Lebenszeit $\tau_{M n}$ können die Transienten nicht über den gesamten Zeitbereich durch Gleichung 5.1 beschrieben werden.

\subsubsection{Abwandlung des Förstermodells für niedrigdimensionale Systeme}

Um diese Abweichung zwischen den gemessenen Transienten und den mit Gleichung 5.1 berechneten Werten näher zu verstehen, wird zunächst das Förstermodell 
näher betrachtet. Das Integral $J\left(t, R_{g}\right)$ in Gleichung 5.1 ist im Modell gegeben durch

$$
J\left(t, R_{g}\right)=\int_{0}^{R_{g}} \exp \left(-\left(\frac{R_{0}}{R}\right)^{6} \frac{t}{\tau_{M n}}\right) w(R) d R
$$

Es wird demnach im Modell angesetzt, dass die Dipol-Dipol-Wechselwirkung zwischen einem angeregten Manganion und einem Killerzentrum proportional zu $R^{-6}$ abnimmt, wobei R den Abstand zwischen Manganion und Killerzentrum im Kristall bezeichnet. Bei einer statistischen Verteilung der Manganionen und Killerzentren in einem ausgedehnten Volumenkristall ist die Wahrscheinlichkeitsdichte $w(R)$, ein Manganion in einem bestimmten Abstand $\mathrm{R}$ zu einem Killerzentrum vorzufinden, gegeben durch

$$
w(R) d R=\frac{4 \pi R^{2}}{V} d R
$$

Dabei bezeichnet $\mathrm{V}$ das Volumen des Kristalls, der als kugelförmig angenommen wird. Es wird folglich eine homogene Verteilung der Manganionen angenommen und über alle drei Raumrichtungen integriert. Werden, wie in diesem Experiment, Wirtskristalle betrachtet, deren Ausdehnung in mindestens eine Raumrichtung gering gegenüber den typischen Wechselwirkungslängen ist, ist die von Förster getroffene Annahme der homogenen Verteilung von Manganionen und Killezentren in einem kugelförmigen Kristall nicht mehr erfüllt. Entsprechend muss die Wahrscheinlichkeitsdichte $w(R)$ angepasst werden. Für ein zweidimensionales (2D) System der Fläche $A=\pi R_{g}^{2}$ reduziert sich die Wahrscheinlichkeitsdichte zu

$$
w(R) d R=\frac{2 \pi R}{A} d R
$$

für ein eindimensionales (1D) System der Länge $R_{g}$ entsprechend zu

$$
w(R) d R=\frac{2}{R_{g}} d R
$$

Nun lassen sich, analog zur Herleitung von Förster, die Gleichungen 5.1 und 5.2 im Limes $R_{g} \rightarrow \infty$ und $N \rightarrow \infty$ lösen. Es ergibt sich ein allgemeiner Ausdruck für den Förster-Energietransfer in Systemen unterschiedlicher Dimensionalität. Die 

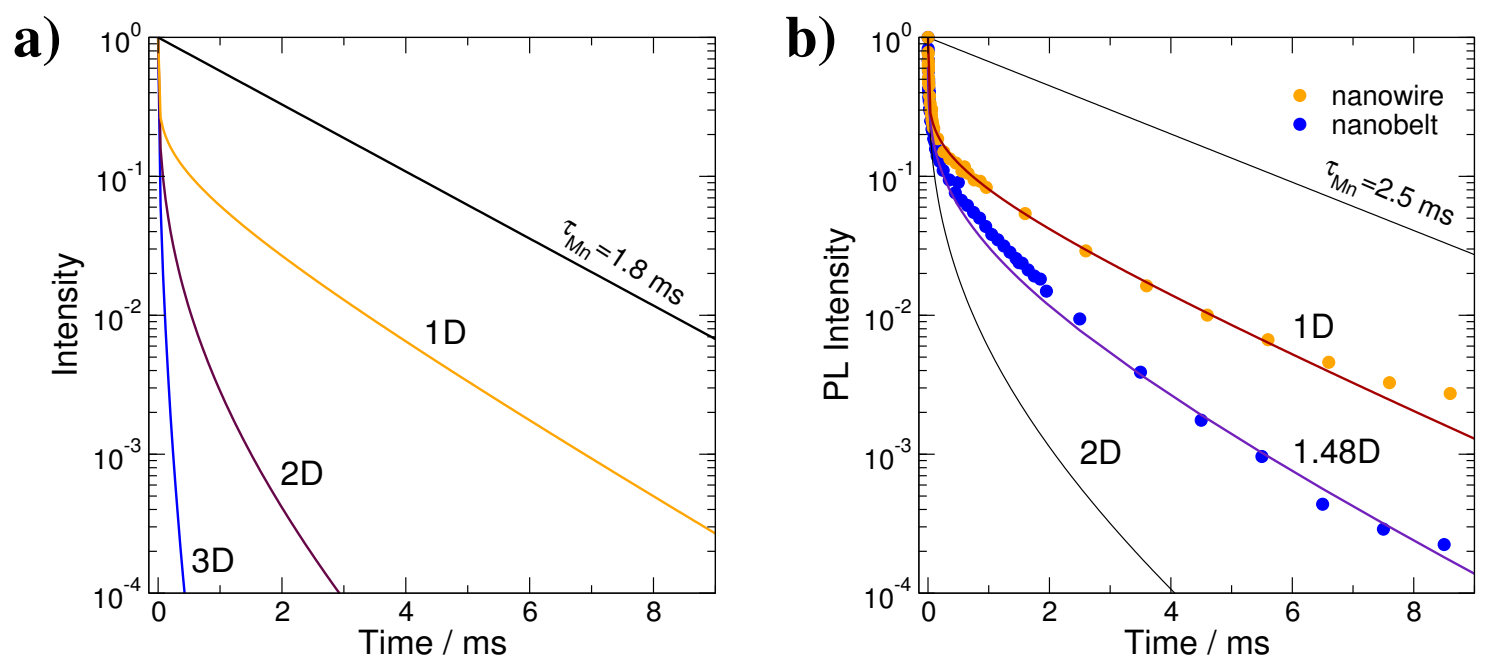

Abbildung 5.5: a) Erwartetes zeitliches Abklingen einer Lumineszenz nach einer Modifikation des Förstertransfers für eindimensionale (1D), zweidimensionale (2D) und dreidimensionale (3D) Systeme. Die Transienten wurden mit Gleichung 5.6 berechnet. Zum Vergleich ist die intrinsische Lebenszeit der Lumineszenz von $\tau_{M n}=1,8$ ms aufgetragen. b) Gemessene Transienten der $\mathrm{Mn}^{2+}$ $3 d^{5}$-Lumineszenz in Zinksulfid-Nanodrähten und -bändern mit einer Mangankonzentration von $1 \cdot 10^{-6}$ at.\%. Die durchgezogenen Linien zeigen eine Anpasung der Messdaten mit Gleichung 5.6.

Transienten lassen sich demnach durch folgenden Ausdruck beschreiben:

$$
\begin{aligned}
\left\langle\frac{I(t)}{I_{0}}\right\rangle & =\exp \left(-\frac{t}{\tau_{M n}}\right)\left[J\left(t, R_{g}\right)\right]^{N} \\
& =\exp \left(-\frac{t}{\tau_{M n}}\right) \exp \left(-\alpha n^{D} R_{0}^{D}\left(\frac{t}{\tau_{M n}}\right)^{\frac{D}{6}}\right) .
\end{aligned}
$$

Dabei bezeichnet $D=1,2,3$ die Dimensionalität des Systems, $n=N / R_{g}$ die Liniendichte von Killerzentren und $\alpha$ nimmt Werte von $\sqrt{\pi}, 1,354$ und 1,129 für den drei-, zwei- und eindimensionalen Fall an. Im Folgenden wird gezeigt, dass die Dimensionalität in dem oben gegebenen Ausdruck durch die Ausdehnung des Kristalls im Verhältnis zu dem mittleren Abstand der Manganionen und Killerzentren bestimmt wird.

Abbildung 5.5 a) zeigt den starken Einfluss der Dimensionalität auf das zeitliche Abklingen in einem Förstertransfer, wobei identische Werte für $\mathrm{n}$ und $\tau_{M n}$ angenommen wurden. Mit kleiner werdender Dimensionalität lassen sich zwei charakteristische Veränderungen der Transienten beobachten. Einerseits prägt sich das 
nichtexponetielle Verhalten der Transienten stärker aus, andererseits klingt die Lumineszenz langsamer ab. Dies lässt sich dadurch verstehen, dass mit sinkender Dimensionalität die relative Anzahl isolierter Manganionen zunimmt. Da von diesen aus kein Energietransfer zu Killerzentren stattfinden kann, werden die Transienten bei großen Zeiten durch ein langsames Abklingen dominiert, dessen Zeitkonstante sich der intrinsischen Lebenszeit annähert. Dieses Verhalten ist bei niedrigen Dimensionen entsprechend stärker ausgeprägt.

Abbildung 5.5 b) zeigt die gleichen Transienten der Manganlumineszenz in Zinksulfid-Nanodrähten und Nanobändern mit einem Mangangehalt von 4 . $10^{-6}$ at.\%, wie sie bereits in Abbildung 5.4 b) gezeigt sind. Die durchgezogenen Linien wurden mit Gleichung 5.6 angepasst. Es zeigt sich, dass die Transienten der Nanodrähte sehr gut mit dem abgewandelten Förstermodell für $\mathrm{D}=1$ beschrieben werden können. Dabei wurde die charakteristische Wechselwirkungslängen zu $R_{0}=4,85 \mathrm{~nm}$ [Che07], die intrinsische Lebenszeit zu $\tau_{M n}=2,5 \mathrm{~ms}$ und die Liniendefektdichte zu 0, $45 \mathrm{~nm}^{-1}$ angesetzt. Wird ein mittlerer Durchmesser der Nanodrähte von $100 \mathrm{~nm}$ angesetzt, so entspricht diese Defektdichte einem mittlerem Abstand der Killerzentren von $25 \mathrm{~nm}$. Der Abstand der Manganionen beträgt, wie oben beschrieben, $200 \mathrm{~nm}$ und ist damit etwa eine Größenordnung höher. Aufgrund des Verhältnises zwischen Nanodrahtdurchmesser, mittlerem Killerzentrenund Manganionenabstand kann also davon ausgegangen werden, dass ein Energietransfer vorwiegend entlang der Nanodrahtachse stattfindet. Das Verhältnis von mittlerem Killerzentren- und Manganionenabstand von $\approx 10$ lässt weiterhin auf einen präferenziellen Energietransfer von angeregten Manganionen zu Killerzentren schließen, was wiederum den Försteransatz rechtfertigt.

Der Transient der $\mathrm{Mn}^{2+}$-intra-3d-Lumineszenz in Nanobändern mit identischer Mangankonzentration wie in den zuvor untersuchten Nanodrähten lässt sich, unter Annahme identischer Werte für $\tau_{M n}, R_{0}$ und $n$, weder mit $\mathrm{D}=1$, noch mit $\mathrm{D}=2$ beschreiben. Es zeigt sich vielmehr, dass der Transient im Bereich zwischen den beiden berechneten Transienten für $\mathrm{D}=1$ und $\mathrm{D}=2$ verläuft. Um den Transienten der Nanobänder dennoch mit Gleichung 5.6 zu beschreiben wurden zur Anpassung auch nicht ganzzahlige Dimensionen zugelassen. Dazu wurden zunächst Werte für $\alpha$ als Funktion von D interpoliert. Die Anpassung ergibt einen Wert für D von 1,48. Dieses Ergebnis lässt sich wie folgt verstehen: Zwischen angeregten Mangan- 
ionen und Killerzentren in der Fläche der Nanobändern kann ein Energietransfer stattfinden; dies trägt zu einem 2D-Verhalten der Transienten bei. Da die Bänder mit einer Breite von 10 - $25 \mu \mathrm{m}$ nicht als unendlich ausgedehnte Schicht aufgefasst werden können, kommt es durch Manganionen an den Seiten der Bänder zu einem 1D-Beitrag zu den Transienten. Gemessen wird die Überlagerung beider Fälle.

Die Anpassung der Transienten der $\mathrm{Mn}^{2+}$-intra-3d-Lumineszenz in ZnS:MnNanodrähten und -bändern mit Gleichung 5.1 ergibt einen Wert für die intrinsische Lebenszeit der Manganlumineszenz von $\tau_{M n}=2,5 \mathrm{~ms}$. Dieser Wert ist deutlich größer als der in der Literatur angegebene Wert von 1,8 ms [Gum81]. In der Dipolnäherug ist die Übergangswahrscheinlichkeit proportional zum Brechungsindex $\mathrm{n}_{r}$ eines Materials. Folglich ist $\tau_{M n}$ proportional $\mathrm{zu} \frac{1}{n_{r}}$. Da die Nanodrähte und Nanobänder mindestens in einer Richtung eine geringere Ausdehnung als die Wellenlänge der Manganlumineszenz haben wird das Lichtwellenfeld nicht ausschließlich in den Nanodrähten geführt, sondern teilweise im umgebenden Vakuum. Es muss also ein relativer Brechungsindex zwischen dem Volumenwert von 2,5 in ZinksulfidVolumenkristallen und 1 im Vakuum angenommen werden, was zu einer effektiven Verkürzung der Lebenszeit führt. Damit ist die verkürzte Lebenszeit der intrinsischen Manganlumineszenz als Dimensionseffekt zu verstehen.

Abbildung 5.6 zeigt Transienten der Manganlumineszenz in ZinksulfidNanostrukturen mit höherer Mangankonzentration. Hier ist der mittlere Abstand zwischen den Manganionen im gleichen Bereich (Abbildung 5.6 a)) bzw. deutlich größer (Abbildung 5.6 b)) als der Abstand der Killerzentren. Folglich kann ein Energietransfers zwischen Manganionen nicht mehr ausgeschlossen werden. Dieser Energietransfer lässt sich empirisch dadurch beschreiben, dass in dem zweiten Exponentialterm in Gleichung 5.6 eine andere Zeitkonstante $\tau_{\text {migr }}$ verwendet wird [DV92]. Diese wird kleiner, je größer die Mangankonzentration ist und ist somit ein Maß für die Effektivität des Energietransfers im Mangansystem. Die Abwandlung von Gleichung 5.6 für hohe Mangankonzentrationen ergibt folglich

$$
\left\langle\frac{I(t)}{I_{0}}\right\rangle=\exp \left(-\frac{t}{\tau_{M n}}\right) \exp \left(-\alpha n^{D} R_{0}^{D}\left(\frac{t}{\tau_{\text {migr }}}\right)^{\frac{D}{6}}\right) .
$$

Die Transienten, die in Abbildung 5.6 a) dargestellt sind, wurden an Nanostrukturen mit einer Mangankonzentrationen von $3,2 \cdot 10^{-2}$ at.\% aufgenommen, was einem 

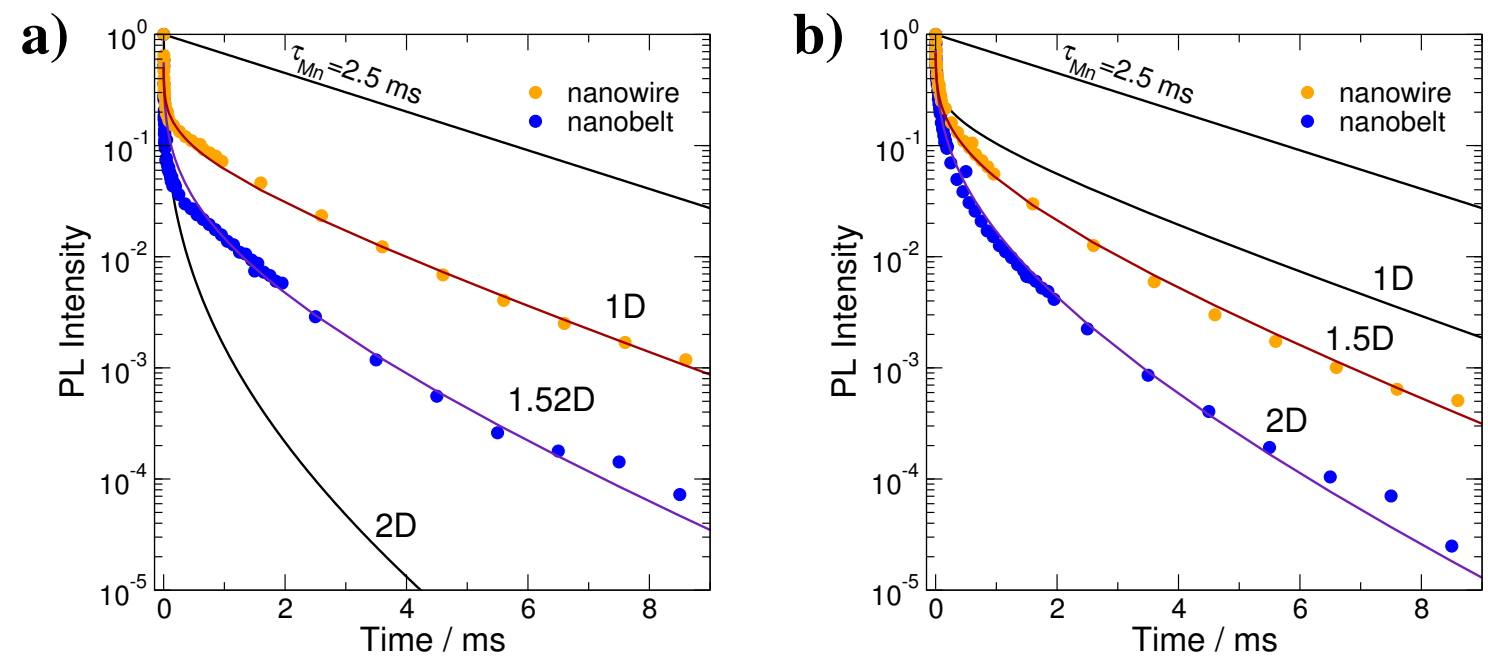

Abbildung 5.6: Gemessene Transienten der $\mathrm{Mn}^{2+}-3 d^{5}$-Lumineszenz in Zinksulfid-Nanodrähten und -bändern mit einer Mangankonzentration von 3,2 $10^{-2}$ at.\% (a)) und 4 at.\% (b)). Die durchgezogenen Linien zeigen eine Anpasung der Messdaten mit Gleichung 5.7.

mittlerem Abstand der Manganionen von $10 \mathrm{~nm}$ entspricht. Die durchgezogenen Linien zeigen eine Anpassung der Messwerte mit Gleichung 5.7, wobei für $R_{0}, n$, und $\tau_{M n}$ die selben Werte wie für die geringe Mangankonzentration eingesetzt, und $\tau_{\text {migr }}$ und D angepasst wurden. Es ergibt sich für die Transienten der Nanodähte und Nanobänder der gleiche Wert für $\tau_{m i g r}=1,2 \mathrm{~ms}$. Dass sich hier eine kürzere Zeitkonstante ergibt als die intrinsische Lebenszeit von 2,5 ms, weist auf einen Energietransfer im Mangansystem hin, wie es aufgrund der vergleichbaren Dichte von Manganionen und Killerzentren zu erwarten war.

Für D ergibt sich aus der Anpassung der Transienten ein Wert von 1 für die Nanodrähte und ein Wert von 1,52 für die Nanobänder. Obgleich die Mangankonzentration bei diesen Proben ungefähr vier Größenordnungen höher ist als bei den zuvor untersuchten Proben, bleiben die angepassten Dimensionsfaktoren D nahezu identisch. Auch die Dichte der Killerzentren bleibt identisch. Dies zeigt, dass die Ausdehnung und Morphologie des Wirtskristalls sowie die Verteilung der Killerzentren darin, entsprechend Gleichung 5.6, die Dimensionalität des Förstertransfers bestimmen.

Abbildung 5.6 b) zeigt die Transienten der Nanodrähte und -bänder mit der höchsten Mangankonzentration von 4 at.\%. Die Anpassung der Transienten er- 
folgte analog mit Gleichung 5.7. Allerdings ließen sich die Transienten nicht, wie zunächst erwartet, ausschließlich durch eine Verkleinerung von $\tau_{m i g r}$ anpassen. Es war zusätzlich notwendig, den Parameter der Defektdichte n zu variieren. Dies lässt sich wie folgt verstehen: Um die hohe Mangankonzentration in den Proben zu erlangen war eine Implantation mit entsprechend hoher Fluenz notwendig. Unter der Annahme, dass sich Zinksulfid bei der Ionenimplantation ähnlich verhält wie Zinkoxid, was zumindest aufgrund der gleichen Bindungsionizität und einem vergleichbarem Schmelzpunkt gegeben ist, sollte die Entstehung typischer Defekten bei ähnlichen Ionenfluenzen und -massen einsetzen. Für Zinkoxid konnte gezeigt werden, dass eine Bestrahlung mit vergleichbarer Ionenfluenz und -masse, wie sie bei den niedrigen Mangankonzentrationen verwendet wurden, in den Bereich 1 und 2 der Defektentstehung fällt [Lor05] (vgl. Abschnitt 4.1.3). Die hohe Mangankonzentration von 4 at.\% ist allerdings im Bereich 3 der Defektentstehung anzusiedeln - der Entstehung von Defektkomplexen. Diese Defektkomplexe sind üblicherweise wesentlich stabiler, heilen im Gegensatz zu Primärdefekten erst bei deutlich höheren Temperaturen aus und können als zusätzliche Killerzentren wirken. Entsprechend wurde bei der Anpassung der Transienten zusätzlich der Parameter der Defektdichte n variiert. Die entsprechenden Anpassungen sind in Abbildung 5.6 b) als durchgezogene Linien aufgetragen. Für $\tau_{\text {migr }}$ ergibt sich ein kleinerer Wert von $\approx 1 \mathrm{~ms}$, was entsprechend der höheren Mangankonzentration zu verstehen ist. Für n ergibt sich ein Wert von $0,34 \mathrm{~nm}^{-1}$, was einer erhöhten Dichte von Killerzentren in dem Material entspricht und vermutlich auf Defektkomplexe zurückzuführen ist. Der Dimensionsparameter D ergibt sich aus der Anpassung zu 2 und 1,5 für Nanobänder und -drähte, was eine Veränderung gegenüber den Anpassungen der Transienten mit geringerer Mangankonzentrationen bedeutet. Dies zeigt erneut, dass ein Wechselspiel zwischen der Ausdehnung und Morphologie des Wirtskristalls und der Konzentration der Killerzentren die Dimensionalität des Förstertransfers bestimmen.

\subsection{Intra-4f-Lumineszenz im System ZnO:RE}

Die Lumineszenz von Seltenen Erden im $3^{+}$-Ladungszustand ist durch scharfe Emissionslinien geprägt [Fre31, Hom90]. Ursprung dieser Linien sind Übergänge zwischen 4f-Schalen der Seltenen Erdionen, die durch die besetzten 5s und 5d Schalen stark 
abgeschirmt sind. Aufgrund dieser Abschirmung ist die spektrale Position der Emissionsbanden nahezu unabhängig vom Wirtskristall. Emissionslinien von Seltenen Erden lassen sich über den gesamten spektralen Bereich, vom Ultraviolett (Gadolinium) bis ins Infrarote (Erbium), finden. Diese intra-4f-Übergänge sind, genauso wie die im letzten Abschnitt diskutierten intra-3d-Übergänge, nach der Laporteschen Regel für das freie Ion paritätsverboten. Eingebaut in einen Wirtskristall wird dieses Verbot aufgrund einer Wechselwirkung zwischen Seltenem Erdion und dem Kristallfeld teilweise aufgehoben [Vle37]. Für die teilweise erlaubten Übergänge sind lange Lebenszeiten charakteristisch. Durch die langen Lebenszeiten und die spektrale Schärfe finden mit Seltenen Erden dotierte, transparente Kristalle Anwendung als optisch aktives Medium in Lasern (z.B. Nd:YAG). Dieses Prinzip auf der Nanometerskala zu realisieren ist von großem Interesse für Anwendungen in der Mikroanalytik oder der optischen Datenspeicherung.

Wie bereits in Abschnitt 4.1 beschrieben, wurden Zinkoxid-Nanodrähte mit Seltenen Erden implantiert. Der Vorteil dieses Systems ist, dass die Nanodrähte zugleich optisch aktives Medium und, aufgrund des großen Brechungsindexes, auch Resonator sein können. In der Literatur sind bereits einige Arbeiten zu Nanodraht-basierten Lasern zu finden [Hua01a, Joh02, Joh03]. Die dort aufgeführte Laseraktivität beruht auf Band-zu-Band-Übergängen oder der Bildung eines Elektron-Loch-Plasmas, wobei vergleichsweise hohe Laserschwellwerte auftreten.

Im Folgenden wird zunächt die optische Aktivierung von Ytterbium, Thulium und Europium in Zinkoxid-Nanodähten untersucht. Die am stärksten ausgeprägte intra4f-Lumineszenz von Ytterbium wird anschließend näher untersucht.

\subsubsection{Aktivierung der intra-4f-Lumineszenz}

Die optische Aktivierung von Seltenen Erden im $3^{+}$-Ladungszustand in II-VIHalbleitern ist wesentlich schwerer zu verstehen, als die in Abschnitt 5.1 diskutierte Aktivierung von Übergangsmetallen. In der Literatur wurde gezeigt, dass Seltene Erden im Kristallgitter Gruppe-II-Plätze einnehmen [Rit06], wo sie entsprechend $2^{+}$-Ionen ersetzen. Um die brillianten intra-4f-Übergänge zu zeigen, müssen die Seltenen Erden jedoch einen $3^{+}$-Ladungszustand haben, wozu eine Ladungskompensation notwendig ist [Boy88, Hom90]. Elektronspinresonanz- sowie Lumineszenz- 
a)

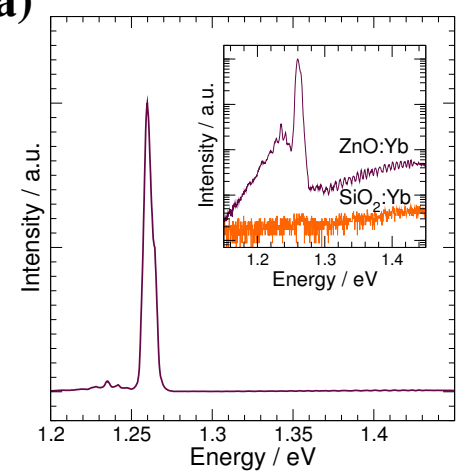

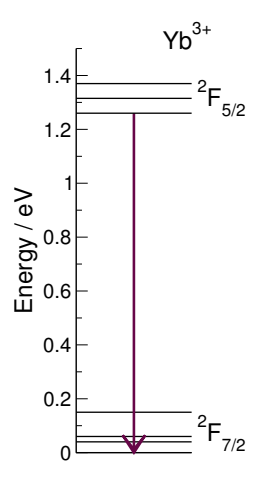

b)

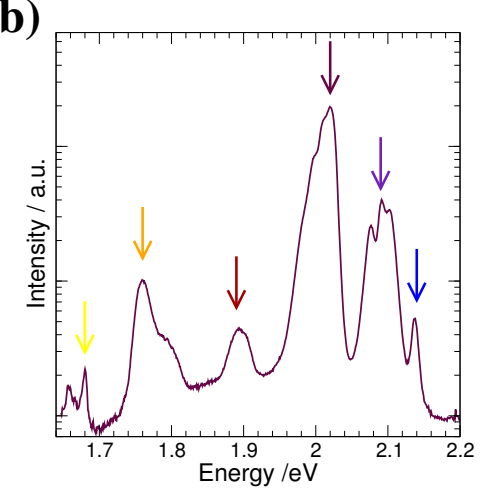

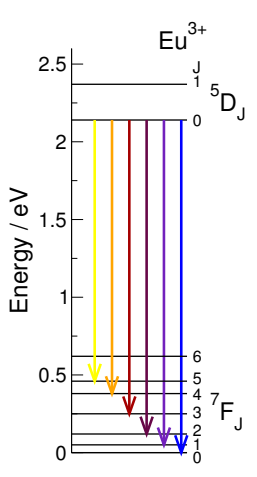

Abbildung 5.7: Lumineszenzspektren von Zinkoxid-Nanodrähten die mit Ytterbium und Europium implantiert und anschließend bei $700{ }^{\circ} \mathrm{C}$ getempert wurden sowie die Zuordnung der Lumineszenzbanden zu den entsprechenden intra-4f-Übergängen. Alle Spektren wurden bei einer Probentemperarur von $15 \mathrm{~K}$ aufgenommen. a) Das Spektrum der Probe Yb_2, die mit $\approx 1,1$ at.\% Ytterbium implantiert wurde zeigt den typischen ${ }^{2} \mathrm{~F}_{5 / 2} \rightarrow{ }^{2} \mathrm{~F}_{7 / 2}$ Übergang. Im Einsatz ist das gleiche Spektrum auf einer logarithmischen Skala zusammen mit dem Spektrum eines Ytterbiumimplantierten Siliziumsubstrats dargestellt. b) Im Lumineszenzspektrum der Probe Eu_3, die mit $\approx 3,3$ at.\% Europium implantiert wurde, konnten die Übergänge ${ }^{5} \mathrm{D}_{0} \rightarrow{ }^{7} \mathrm{~F}_{\mathrm{J}}$ beobachtet werden.

messungen zeigen allerdings, dass Seltene Erden in II-VI-Halbleitern zumeist im $3^{+}$-Ladungszustand eingebaut werden [Boy88]. Die Ladungskompensation kann eigentlich nur über Kristalldefekte in der direkten Umgebung der Seltenen Erden stattfinden. Dafür spricht in jedem Fall, dass die Kristallqualität keinen großen Einfluss auf die intra-4f-Lumineszenz hat. So konnte gezeigt werden, dass die intra4f-Lumineszenz bei resonanter Anregung von einer Schädigung des Kristalls unbeeinflusst bleibt [Hom92]. Insbesondere treten keine anderen Starkniveaus durch veränderte lokale Umgebungen auf. Lediglich bei nichtresonanter Anregung konnte eine geringere Effizienz der Lumineszenz beobachtet werden, da der Anregungskanal über den Kristall durch die Schädigung des Gitters eingeschränkt wurde. Die Komplexe aus Seltenen Erden und Defekten können wie stabile Moleküle betrachtet werden, die in der Halbleitermatrix eingebettet sind.

Abbildung 5.7 zeigt Lumineszenzspektren von Zinkoxid-Nanodrähten, die mit Ytterbium bzw. Europium implantiert und anschließend für $30 \mathrm{~min}$. bei $700{ }^{\circ} \mathrm{C}$ getempert wurden. Strukturelle Untersuchungen an den Proben wurden in Abschnitt 4.1 vorgestellt. Sowohl Europium als auch Ytterbium haben aufgrund der halb bzw. vollständig gefüllten $4 \mathrm{f}$-Schale einen $2^{+}$-Ladungszustand in II-VI- 
Halbleitern [Boy88, Swi89, Che00]. Allerdings reicht die Anregungsenergie bei den Lumineszenzuntersuchungen für eine Photoionisation in den $3^{+}$-Zustand aus.

Abbildung 5.7 a) zeigt ein Photolumineszenzspektrum der Probe Yb_2 (1,1 at.\% Ytterbium) im Spektralbereich der für das Ytterbium charakteristischen intra4f-Lumineszenz. In der Tat ist eine intensive und schmale Lumineszenzbande bei 1,260 eV zu beobachten. Entsprechend der Literatur wird dieser Bande dem ${ }^{2} F_{5 / 2} \rightarrow{ }^{2} F_{7 / 2}$ Übergang zugeordnet, der auch der einzig mögliche intra-4fÜbergang in $\mathrm{Yb}^{3+}$ ist [Die68, Geb08]. Insbesondere in der logarithmischen Auftragung des Spektrums im Einsatz von Abbildung 5.7 a) ist zu erkennen, dass zu niedriger Energie $(1,235 \mathrm{eV})$ eine weitere Bande folgt. Die Modulation auf dieser Bande ist auf ein apparatives Artefakt in diesem Wellenlängenbereich zurückzuführen. Das Auftreten dieser Bande ist vermutlich auf eine Aufspaltung der ${ }^{2} F_{7 / 2}$ und ${ }^{2} F_{5 / 2}$ in mehrere Starkniveaus durch das umgebende Kristallfeld zurückzuführen. Unter der Annahme, dass die Ytterbiumionen Zinkplätze im Kristallgitter einnehmen, spaltet das $\mathrm{J}=5 / 2$ Niveau dreifach und das $\mathrm{J}=7 / 2$ Niveau vierfach auf. In anderen Halbleitermaterialien werden typischerweise scharfe Übergänge (FWHM $\approx 1 \mathrm{meV}$ ) aus dem niedrigsten 5/2 Niveau in die vier $7 / 2$ Niveaus beobachtet [Cas06, Sat04, Kör88]. Die Breite der Aufspaltung wurde in anderen Materialien $\mathrm{zu} \approx 100 \mathrm{meV}$ bestimmt [Bou07], sodass die zweite beobachtete Bande durchaus auf einen Übergang in ein anderes Starkniveau zurückgeführt werden kann. Die hier beobachtete Linienbreite der intra-4f-Übergänge ist mit einer Halbwertsbreite von $\approx 10 \mathrm{meV}$ um einen Faktor 10 größer als in anderen Kristallen beobachtet wurde. Außerdem ist insbesondere die Form der intensivsten Linie stark asymmetrisch. Dies weist darauf hin, dass es aufgrund unterschiedlicher lokaler Umgebungen des Ytterbiums im Zinkoxid zu unterschiedlichen Kristallfeldaufspaltungen kommt.

Um auszuschließen, dass die beobachtete Lumineszenz von Ytterbiumionen in der natürlichen $\mathrm{SiO}_{2}$-Deckschicht des Siliziumsubstrate herrührt, wurden Referenzmessungen an einer auf gleiche Weise präparierten Probe durchgeführt, auf die zuvor keine Zinkoxid-Drähte aufgebracht wurden. Ein entsprechendes Referenzspektrum ist im Einsatz von Abbildung 5.7 a) aufgetragen. Selbst auf logarithmischer Skala ist kein Indiz für eine Lumineszenz in diesem Wellenlängenbereich zu erkennen.

Eine nähere Analyse des Kristallfeldes kann anhand der intra-4f-Lumineszenz des Europiums durchgeführt werden. Europium zeigt typischerweise ein Multiplett an 
Emissionslinien im roten Spektralbereich, wobei die relativen Intensitäten Aufschluss über die Symmetrie des Kristallfeldes geben können. Abbildung 5.7 b) zeigt ein Kathodolumineszenzspektrum der Probe Eu_3 (3,3 at.\% Europium) im spektralen Bereich der intra-4f-Lumineszenz des Europiums. Das beobachtete Multiplett an Emissionsbanden lässt sich den intra-4f-Übergängen aus dem ${ }^{5} \mathrm{D}_{0}$-Niveau in die ${ }^{7} F_{J}$-Niveaus mit $J=0-5$ zuordnen [Car68, Per07, Liu08]. Übergänge aus höheren Niveaus werden nicht beobachtet, was zu der Annahme führt, dass nach dem Energietransfer in das intra-4f-System zunächst eine Relaxation in das ${ }^{5} D_{0}$-Niveau stattfindet [Liu08].

Die intensivste Bande konnte dem Übergang ${ }^{5} D_{0} \rightarrow{ }^{7} F_{2}$ zugeordnet werden. Bei diesem Übergang handelt es sich um einen elektrischen Dipolübergang, der bei einem stark asymmetrischen Kristallfeld besonders intensiv auftritt [Liu08]. Anders verhält es sich mit dem ${ }^{5} D_{0} \rightarrow{ }^{7} F_{1}$ Übergang. Dabei handelt es sich um einen magnetischen Dipolübergang, dessen Übergangswahrscheinlichkeit relativ unabhängig von der Symmetrie des Kristallfeldes ist [Zha05]. Da die Intensität der ${ }^{5} D_{0} \rightarrow{ }^{7} F_{2}$ Bande im Verhältnis größer ist als die der ${ }^{5} D_{0} \rightarrow{ }^{7} F_{1}$ Bande, kann davon ausgegangen werden, dass die typischen Kristallfelder der Europiumionen asymmetrisch sind. Dies ist für Europium auf einem ungestörten Zink-Gitterplatz nicht zu erwarten. Ein weiteres Anzeichen für ein stark asymmetrisches Kristallfeld ist das Auftreten des ${ }^{5} D_{0} \rightarrow{ }^{7} F_{0}$ Übergangs. Dieser unterliegt nach den Auswahlregeln $(\Delta J=0)$ einem besonders starken Verbot [Boy88, Liu08]. Dass er in den Spektren dennoch auftritt, bestätigt die Asymmetrie des Kristallfeldes.

Neben dem Intensitätsverhältnis und dem Auftreten einzelner Übergänge kann auch bei Europium eine Aufspaltung der einzelnen Emissionslinien beobachtet werden. Für die intensivste Bande für $\mathrm{J}=2$ wird eine Aufspaltung in drei Starkniveaus erwartet, wenn das Europiumion einen Zinkplatz in Zinkoxid einnimmt [Liu07]. Diese Aufspaltung wird auch beobachtet. Allerdings zeigt auch der Übergang in das ${ }^{7} F_{1}$-Niveau mindestens eine dreifache Aufspaltung, was bei der Symmetrie eines Zn-Gitterplatzes nicht erwartet wird. Zusammen mit der großen Breite aller Linien ist demnach davon auszugehen, dass mehrere Kristallfelder, d.h. unterschiedliche lokale Umgebungen, zu der Aufspaltung der Linien beitragen [Nye03, Liu07].

Abbildung 5.8 zeigt ein Photolumineszenzspektrum von Zinkoxid-Nanodähten, die mit $\approx 1,1$ at. $\%$ Thulium implantiert und bei $700{ }^{\circ} \mathrm{C}$ getempert wurden. Der beobach- 

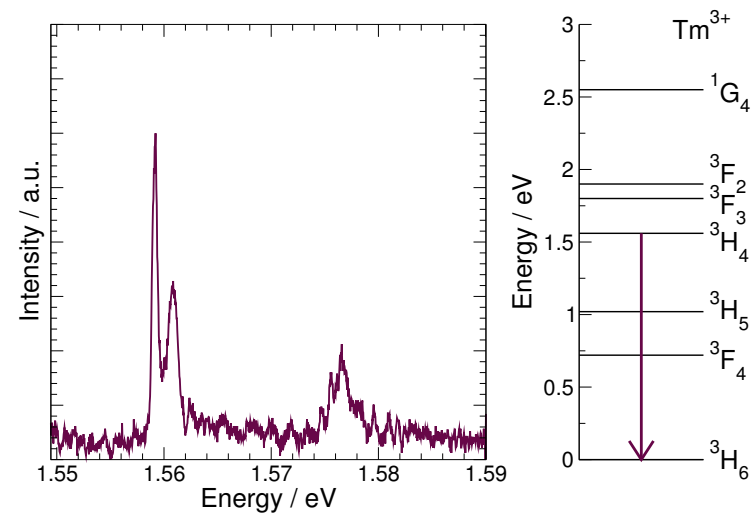

Abbildung 5.8: Lumineszenzspektrum der Probe Tm_2 $(\approx 1,1$ at.\% Thulium, getempert bei $700{ }^{\circ} \mathrm{C}$ ). Das Spektrum wurden bei einer Probentemperarur von $15 \mathrm{~K}$ aufgenommen. Im rechten Teil der Abbildung ist das entsprechende Dieckediagramm dargestellt. Die beobachtete Lumineszenz wurde dem Übergang ${ }^{3} \mathrm{H}_{4} \rightarrow{ }^{3} \mathrm{H}_{6}$ zugeordnet.

tete Übergang wird aufgrund seiner energetischen Lage dem ${ }^{3} \mathrm{H}_{4} \rightarrow{ }^{3} \mathrm{H}_{6}$ Übergang zugeordnet [Bac93]. Zwar ist demnach auch eine Identifizierung als ${ }^{1} \mathrm{G}_{4} \rightarrow{ }^{3} \mathrm{H}_{5}$ möglich, da aber keine weiteren Übergänge aus dem ${ }^{1} \mathrm{G}_{4}$-Niveau beobachtet werden, ist die getroffene Zuordnung wahrscheinlicher. Im Übrigen werden Übergänge aus dem ${ }^{1} \mathrm{G}_{4}$-Niveau in Zinkoxid in den meisten Fällen nicht beobachtet [Rit03, Mon04]. Im Lumineszenzspektrum sind zwei scharfe Linien bei $\approx 1,560 \mathrm{eV}$ sowie eine breitere, schwache Bande bei $\approx 1,577 \mathrm{eV}$ zu erkennen. Diese werden wiederum auf eine Aufspaltung des ${ }^{3} H_{6}$ in unterschiedliche Starklevel sowie auf unterschiedliche Kristallfelder zurückgeführt [Mon06, Per04].

Zusammenfassend ist aufgrund der Breite, der unterschiedlichen Aufspaltung sowie dem Intensitätsverhältnis zwischen einzelnen Linien davon auszugehen, dass sich ein Großteil der optisch aktiven Seltenen Erdionen in unterschiedlichen, asymmetrischen Kristallfeldern befindet. Zwar wurde in der Literatur gezeigt, dass ein Großteil der Seltenen Erdionen nach Ionenimplantation und einer Temperaturbehandlung Zinkgitterplätze einnehmen [Rit03, Rit06], allerdings wurde auch gezeigt, dass nur ein kleiner Teil der Seltenen Erdionen optisch aktiv ist [Rit03]. Aufgrund der beobachteten geringen Intensität der intra-4f-Lumineszenz und vergleichbarer experimenteller Voraussetzungen kann auch in diesem Experiment davon ausgegangen werden, dass nur ein kleiner Teil der Seltenen Erdionen optisch aktiv ist. 


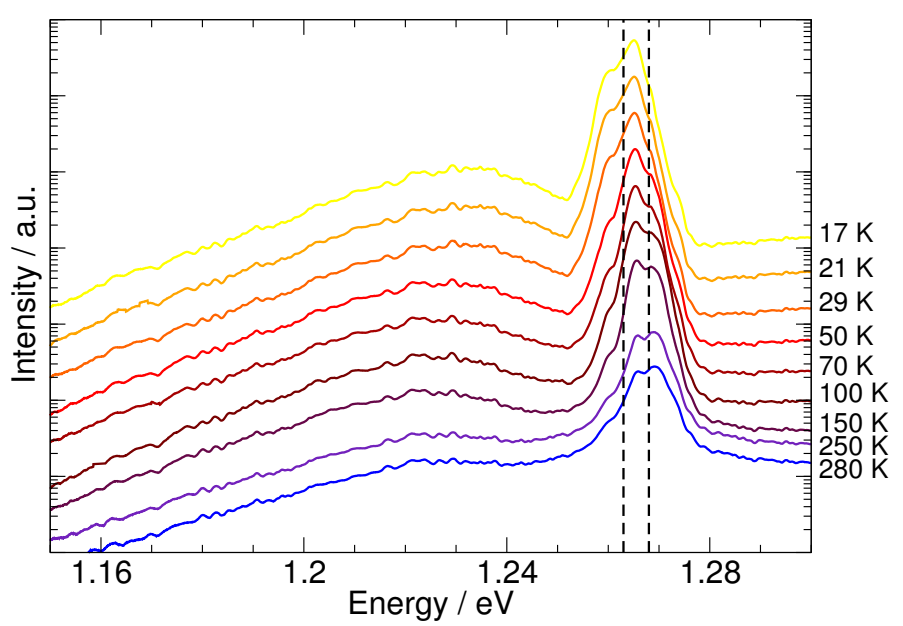

Abbildung 5.9: Lumineszenzspektren der Probe Yb_2 bei unterschiedlichen Temperaturen im spektralen Bereich der $\mathrm{Yb}^{3+}$-intra-4f-Lumineszenz. Die Lumineszenz verschiebt mit steigender Temperatur zu höherer Energie und ist auch bei Raumtemperatur noch zu beobachten.

Die umgebenden unterschiedlichen Kristallfelder stehen vermutlich im Zusammenhang mit der verbleibenden Gitterschädigung (vgl. Abschnitt 4.1).

\subsubsection{Temperaturabhängigkeit der $\mathrm{Yb}^{3+}$-intra-4f-Lumineszenz}

Abbildung 5.9 zeigt eine Serie von Lumineszenzspektren der Probe Yb_2, die bei verschiedenen Probentemperaturen unter der Anregung mit einem HeCd-Laser aufgenommen wurde. Es ist zu erkennen, dass die intensivste Bande bei 1,263 eV bei steigender Temperatur zu höherer Energie verschiebt. Die Modulation auf den Banden ist auf ein apparatives Artefakt zurückzuführen, sodass die spektrale Position in der Mitte der Bande angenommen wird. Die Verschiebung beträgt bis Raumtemperatur $\approx 5 \mathrm{meV}$. In der Literatur wurde diese Verschiebung der thermisch aktivierten Bevölkerung höherer Starklevel des ${ }^{2} F_{5 / 2}$-Niveaus zugeordnet [Lup99]. Der Übergang in die ${ }^{2} F_{7 / 2}$-Grundniveaus erfolgt dann, wie beobachtet, blauverschoben. Eine weitere wichtige Beobachtung aus der Temperaturabhängigkeit der Photolumineszenz ist, dass die intra-4f-Lumineszenz des Ytterbiums auch bei Raumtemperatur noch zu beobachten ist. Dies ist essentiell für potentielle Anwendungen. 
a)

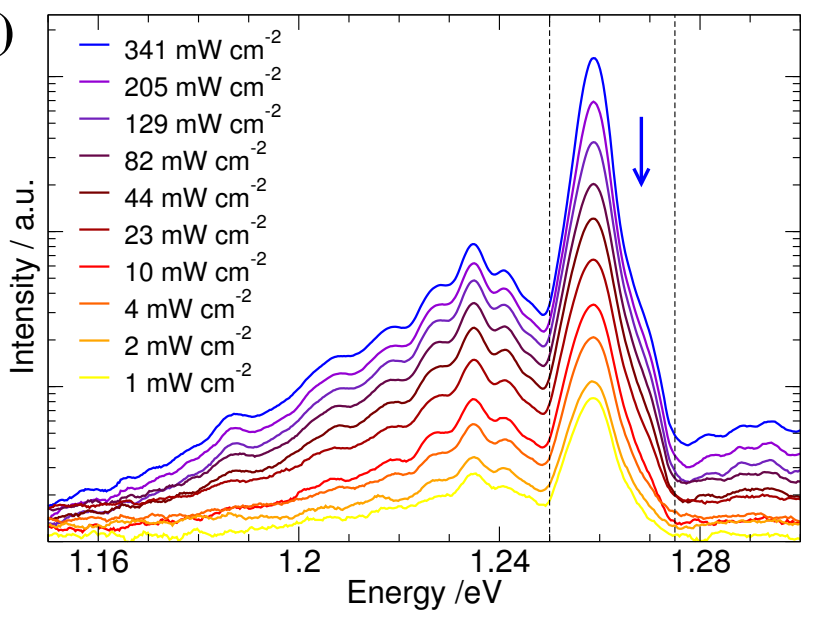

b)

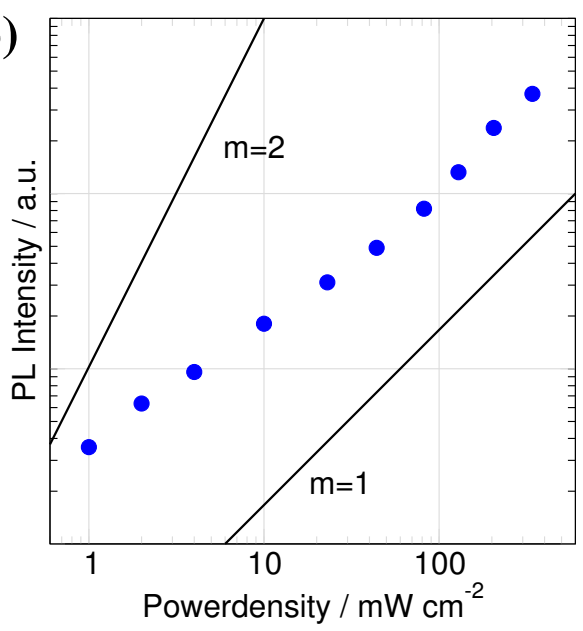

Abbildung 5.10: Untersuchungen der $\mathrm{Yb}^{3+}$-intra-4f-Lumineszenz bei unterschiedlichen Anregungsdichten. a) Lumineszenzspektren der Probe Yb_2 bei unterschiedlichen Anregungsdichten im spektralen Bereich der $\mathrm{Yb}^{3+}$-intra-4f-Lumineszenz, die bei einer Probentemperatur von $15 \mathrm{~K}$ unter Anregung mit einem HeCd-Laser aufgenommen wurden. b) Integrale Intensität im spektralen Bereich von 1,250 eV - 1,275 eV als Funktion der Anregungsdichte.

\subsubsection{Leistungsabhängigkeit der $\mathrm{Yb}^{3+}$-intra-4f-Lumineszenz}

Abbildung 5.10 a) zeigt Lumineszenzspektren der Probe Yb_2, die bei einer Temperatur von $15 \mathrm{~K}$ bei unterschiedlichen, moderaten Anregungsdichten aufgenommen wurden. Die Anregung erfolgte mit einem HeCd-Laser, wobei die Anregungsdichte über einen Neutraldichtefilter variiert wurde. In der logarithmischen Auftragung ist zu erkennen, dass bei steigender Anregungsdichte an der hochenergetischen Flanke der intra-4f-Bande eine weitere Schulter herauswächst (blauer Pfeil). Analog zu den Beobachtungen in den temperaturabhängigen Messungen ist dies vermutlich ebenfalls auf eine zunehmende Besetzung höherer Starkniveaus zurückzuführen.

In Abbildung 5.10 b) ist die integrale Intensität der intra-4f-Bande über den spektralen Bereich von 1,250 eV - 1,275 eV als Funktion der Anregungsdichte doppellogarithmisch aufgetragen. Mit steigender Anregungsdichte nimmt die Intensität der Lumineszenz zunächst unterproportional zu (Steigung kleiner 1 in der doppellogarithmischen Auftragung). Ab einem Schwellwert bei etwa $100 \mathrm{~mW} \mathrm{~cm}{ }^{-2}$ nimmt die Intensität dann schwach überproportional zu (Steigung größer 1 in der doppellogarithmischen Auftragung). Dieses Schwellenverhalten ist relativ schwach ausgeprägt, 
aber dennoch deutlich zu erkennen und lässt im Grunde zwei Interpretationen zu: (1) Bei geringen Anregungsintensitäten werden zunächst Störstellen abgesättigt, wodurch es $\mathrm{zu}$ einer unterproportionalen Intensitätszunahme kommt. Ab dem Schwellwert sind die Defekte abgesättigt und die Intensität wächst linear mit der Anregungsdichte. (2) Alternativ können strahlungslose Rekombinationsmechanismen im intra-4f-System wie Kreuzrelaxationen, intra-4f-Multiphononenübergänge oder Energietransfer zu Killerzentren mit der Anregungsdichte skalieren [Boy88], was zu einer unterproportionalen Zunahme der Intensität bei geringen Anregungsdichten führen kann. Bei höheren Anregungsdichten kann es, begünstigt durch die große Lebenszeit der intra-4f-Übergänge, zu einer Besetzungsinversion und einer einsetzenden stimullierten Emission kommen. Dies resultierende optische Verstärkung würde dann die überproportionale Intensitätszunahme der Lumineszenz erklären. Welcher Prozess Ursache des beobachteten Verhaltens ist, oder ob eine Überlagerung beider Prozesse zu dem beobachteten Verhalten führt, kann aus diesen Untersuchungen nicht geklärt werden.

\subsection{Zusammenfassung}

In diesem Kapitel wurden Untersuchungen an optischen Übergängen von Übergangsmetallen und Seltenen Erden in Zinksulfid bzw. Zinkoxid-Nanodrähten dargestellt.

Es wurde gezeigt, dass Manganionen, die durch Ionenimplantation in einkristalline Zinksulfid-Nanostrukturen eingebracht wurden, nach einer geeigneten Temperaturbehandlung optisch aktiv im $2^{+}$-Zustand auf Zinkgitterplätzen eingebaut sind. Außerdem wurde gezeigt, dass die Lebenszeit der $\mathrm{Mn}^{2+}$-intra-3d-Lumineszenz in Zinksulfid-Nanodrähten ein stark nichtexponetielles Verhalten zeigt. Wie es bereits für ZnS:Mn-Nanopartikel gezeigt wurde, lässt sich dieses nichtexponentielle Abklingen der ${ }^{4} T_{1} \rightarrow{ }^{6} A_{1}$ Lumineszenz, anders als in ZnS:Mn-Volumenkristallen, nicht durch das Förstermodell bescheiben. In einer systematischen Untersuchung an Zinksulfid-Nanostrukturen unterschiedlicher Geometrie, bei der die Mangankonzentration über sechs Größenordnungen variiert wurde, konnte gezeigt werden, dass sowohl die Geometrie der Wirtskristalle als auch die Mangankonzentration einen starken Einfluss auf das zeitliche Abklingverhalten hat. 
Aufbauend auf dem Förstermodell, in dem ein Energietransfer zu Zentren nichtstrahlender Rekombination (Killerzentren) bzw. bei hoher Mangankonzentration auch ein Energietransfer zwischen Manganionen als Ansatz für das nichtexponentielle Abklingen dient, konnte hier ein Modell entwickelt werden, mit dem die gemessenen Transienten konsistent beschrieben werden konnten. Die Idee des entwickelten Modells besteht darin, dass die Effektivität des Energietransfers von angeregten Manganionen zu Killerzentren auch durch die Verteilung der Killerzentren bestimmt wird. Diese Verteilung, die in Försters Berechnungen als statistisch in einem ausgedehnten Volumen angenommen wurde, verändert sich deutlich, wenn die Abmessungen des Wirtskristalls vergleichbar mit dem mittleren Abstand der Killerzentren sind. Entsprechend wurde das Förstermodell um einen Parameter erweitert, der als Dimensionsparameter aufgefasst werden kann.

Die untersuchten Nanostrukturen haben in mindestens einer Raumrichtung eine geringere Ausdehnung als die Wellenlänge der Manganlumineszenz. Folglich kann diese sich nicht mehr frei in dem Kristall ausbreiten, sondern wird zum Teil als evaneszentes Feld außerhalb der Nanostrukturen im Vakuum geführt. Dieser Effekt kann als effektive Verringerung des Brechungsindexes aufgrund der eingeschränkten Dimension aufgefasst werden und resultiert im Experiment in einer vergrößerten intrinsischen Lebenszeit der $\mathrm{Mn}^{2+}$-Lumineszenz.

Es wurde außerdem gezeigt, dass auch Seltene Erdionen, die durch Ionenimplantation in einkristalline Zinkoxid-Nanodrähte eingebracht wurden, nach einer geeigneten Temperaturbehandlung optisch aktiv im $3^{+}$-Zustand sind. Die beobachteten intra-4f-Übergänge weisen durch ihre spektrale Breite und ihre relativen Intensitäten auf unterschiedliche und zum Teil asymmetrische Kristallfelder der Seltenen Erden hin. Dies ist in Übereinstimmung mit der in Abschnitt 4.1 beschriebenen verbleibenden Gitterschädigung.

In temperaturabhängigen Photolumineszenzmessungen an einer mit Ytterbium implantierten Probe konnte gezeigt werden, dass die Lumineszenz auch bei Raumtemperatur noch $\mathrm{zu}$ beobachten ist. Eine spektrale Blauverschiebung mit steigender Temperatur wurde auf die thermisch-aktivierte Bevölkerung höherer Starkniveaus zurückgeführt. In leistungsabhängigen Messungen an der intra-4f-Lumineszenz des Ytterbiums konnte ein Schwellenverhalten bei sehr geringen Leistungsdichten beobachtet werden. Dies kann als Hinweis auf einsetzende stimulierte Emission aufgefasst 
werden. Entsprechend stellen mit Seltenen Erden dotierte Zinkoxid-Nanodrähte ein vielversprechendes System nanodrahtbasierter Laser dar. 


\section{Kapitel 6}

\section{Funktionalisierung von Nanodrähten als elektronische Bauelemente}

\section{1 p-Dotierung von Galliumarsenid-Nanodrähten}

Die Möglichkeit der Dotierung von Halbleiternanodrähten ist essentiell für deren Funktionalisierung als elektronisches und optoelektronisches Bauteil. Die Dotierung von Nanodrähten während der Synthese gestaltet sich aufgrund des Wachstumsprozesses als schwierig (vgl. Kapitel 2.1). Selbst wenn sie glückt, fehlt eine Kontrolle über die Dotierkonzentration. Im Rahmen dieser Arbeit wurde eine alternative Methode verwendet: zur p-Dotierung von Galliumarsenid-Nanodrähten wurden diese nach der Synthese mit Zinkionen implantatiert. Strukturelle Untersuchungen an den implantierten Nanodrähten wurden bereits in Abschnitt 4.2 vorgestellt. Die Prozessierung der Nanodrähte und die elektrischen Messungen sind in Zusammenarbeit mit der Gruppe Tegude, Universität Duisburg durchgeführt wurden.

\subsubsection{Präparation der Proben}

Die Herstellung der verwendeten Nanodrähte wurde bereits in Abschnitt 4.2.1 beschrieben. Leitfähigkeitsmessungen wurden sowohl an mit Zink implantierten als auch an unbehandelten Galliumarsenid-Nanodrähten durchgeführt. Die Implantation resultiert in einer homogenen Volumenkonzentration von $\approx 3 \cdot 10^{19} \mathrm{~cm}^{-3}$ Zinkatomen (vgl. Abbildung 4.13). Anschließend wurden die Nanodrähte bei einer Temperatur von $800{ }^{\circ} \mathrm{C}$ im MOVPE-Reaktor unter TBAs Gegendruck ausgeheilt. Um 
a)

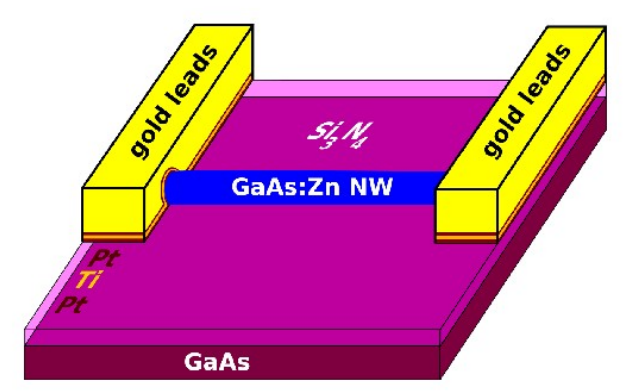

b)

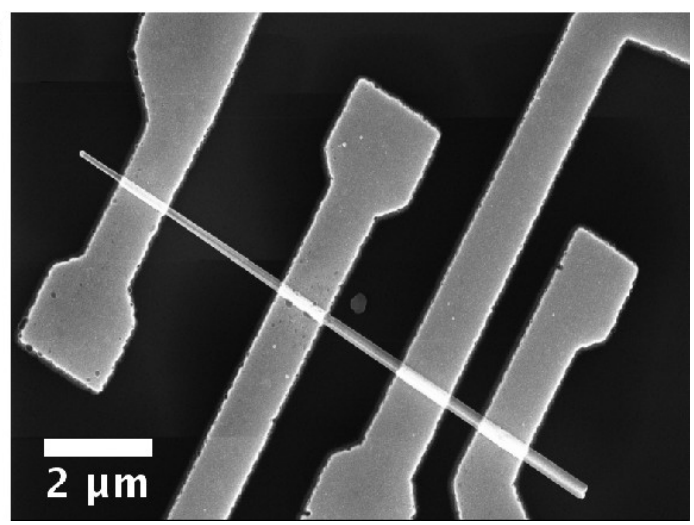

Abbildung 6.1: a) Schema der kontaktierten Galliumarsenid-Nanodrähte. Als Leiterbahnen wurden Standard p-typ Kontakte verwendet: (5 nm Platin, $10 \mathrm{~nm}$ Titan, $5 \mathrm{~nm}$ Platin und $300 \mathrm{~nm}$ Gold). Als Substrat diente Galliumarsenid (i-GaAs), auf das eine isolierende $\mathrm{Si}_{3} \mathrm{~N}_{4}$-Schicht aufgebracht wurde. b) SEM-Aufnahmen eines in TLM-Geometrie kontaktierten GalliumarsenidNanodrahtes.

elektrische Kontakte an die Nanodrähte anzubringen, wurden diese zunächst, wie in Abschnitt 2.2.2 beschrieben, von dem Wachstumssubstrat entfernt und auf einem isolierenden Substrat mit geringer Dichte dispergiert. Bei den isolierenden Substraten handelt es sich um undotierte Galliumarsenid-Kristalle, auf die eine $250 \mathrm{~nm}$ dicke, isolierende $\mathrm{Si}_{3} \mathrm{~N}_{4}$ Schicht aufgebracht wurde. Auf den Substraten wurden mittels optischer Lithographie Messfelder mit makroskopischen Gold-Kontakten aufgebracht, die bei den elektrischen Messungen an einem Spitzenmessplatz adressiert wurden.

An die dispergierten Nanodrähte wurden mittels Elektronenstrahllithographie elektrische Kontakte angebracht (vgl. Abschnitt 2.2.3). Dazu wurde ein Standardschichtpaket für p-typ Kontakte an Galliumarsenid verwendet, das aus $5 \mathrm{~nm}$ Platin, $10 \mathrm{~nm}$ Titan, $5 \mathrm{~nm}$ Platin und $300 \mathrm{~nm}$ Gold besteht. Die Kontakt wurden in einer TLM-Geometrie (transission line meassurement) angeordnet. Ein in der beschriebenen Geometrie kontaktierter Nanodraht ist in Abbildung 6.1 a) schematisch und in Abbildung b) als SEM-Aufnahme zu sehen.

Auf diese Weise wurden insgesamt 22 Nanodrähte vermessen. Die Ergebnisse der Messungen sind im Wesentlichen konsistent. Entsprechend werden im Folgenden repräsentative Strom-Spannungskennlinien eines nichtimplantierten Nanodrahtes, 

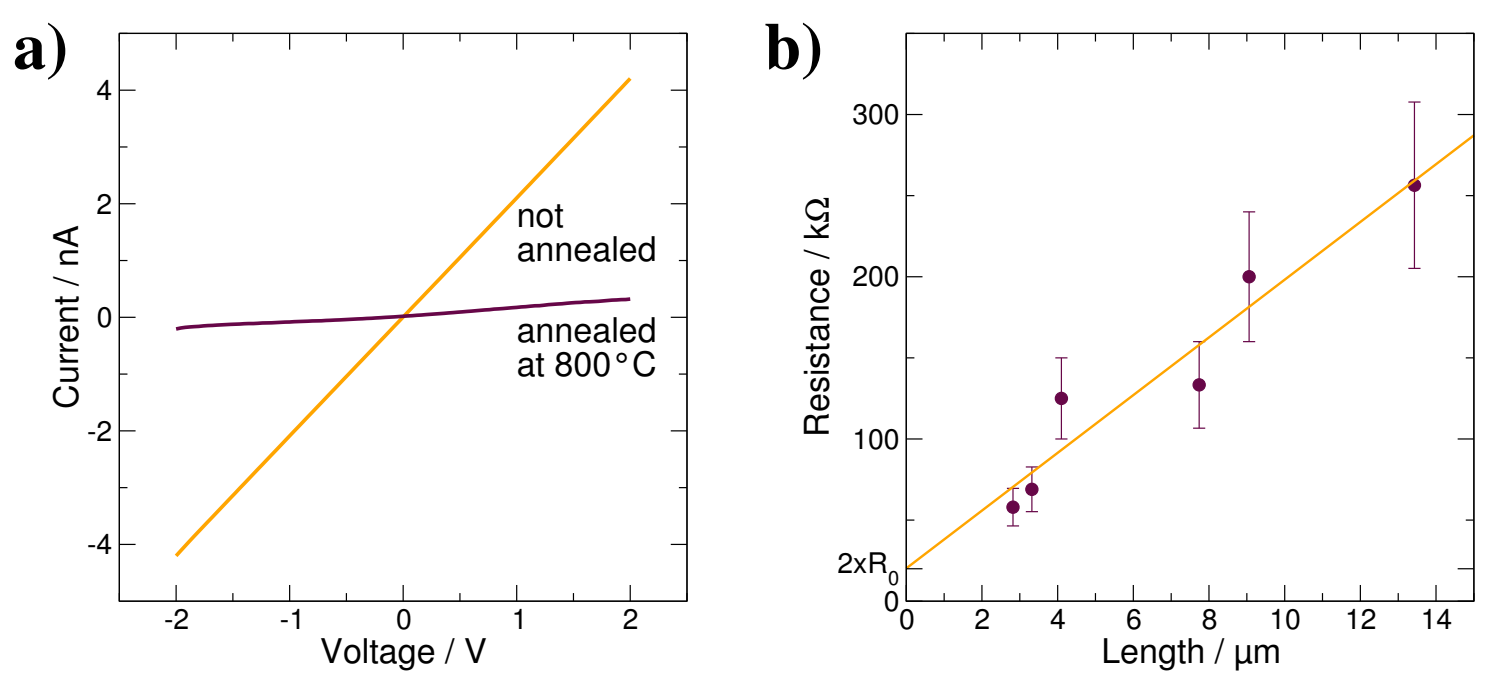

Abbildung 6.2: a) Strom-Spannungskennlinien von Galliumarsenid-Nanodrähten, wie sie synthetisiert wurden und nach einer Temperaturbehandlung im MOVPE-Reaktor bei $800{ }^{\circ} \mathrm{C} . \mathbf{b}$ ) Widerstände von verschiedenen Abschnitten eines Galliumarsenid-Nanodrahtes als Funktion der Länge des vermessenen Abschnitts. Der Achsenabschnitt hat die Größe des doppelten Kontaktwiderstandes.

eines nichtimplantierten und getemperten Nanodrahtes sowie eines implantierten und getemperten Nanodrahtes vorgestellt.

\subsubsection{Leitfähigkeitsmessungen Galliumarsenid-Nanodrähten} an

nichtimplantierten

Abbildung 6.2 zeigt die Kennlinie eines nichtimplantierten GalliumarsenidNanodrahtes zusammen mit der Kennlinie eines Galliumarsenid-Nanodrahtes der einer Temperaturbehandlung $\left(800{ }^{\circ} \mathrm{C}, 30 \mathrm{~min}\right)$ unterzogen wurde. Der Abstand der Kontakte war bei beiden Kennlinien identisch und betrug $\approx 1 \mu \mathrm{m}$. Beide Kennlinien zeigen eine lineare Charakteristik, was auf einen ohmschen Kontakt zwischen Leiterbahn und Nanodraht hinweist.

Bei einer Spannung von $4 \mathrm{~V}$ fließt durch die Nanodrähte ein Strom von $4 \mathrm{nA}$, was einem Widerstand von 0,5 G $\Omega$ entspricht. Nach einer Temperaturbehandlung der Drähte bei $800{ }^{\circ} \mathrm{C}$ im MOVPE-Reaktor steigt der Widerstand bis auf $10 \mathrm{G} \Omega$ an. Da die Temperaturbehandlung bei einer deutlich höheren Temperatur durchgeführt wurde als der Wachstumsprozess der Nanodrähte $\left(450{ }^{\circ} \mathrm{C}\right)$, ist eine Verbesserung 


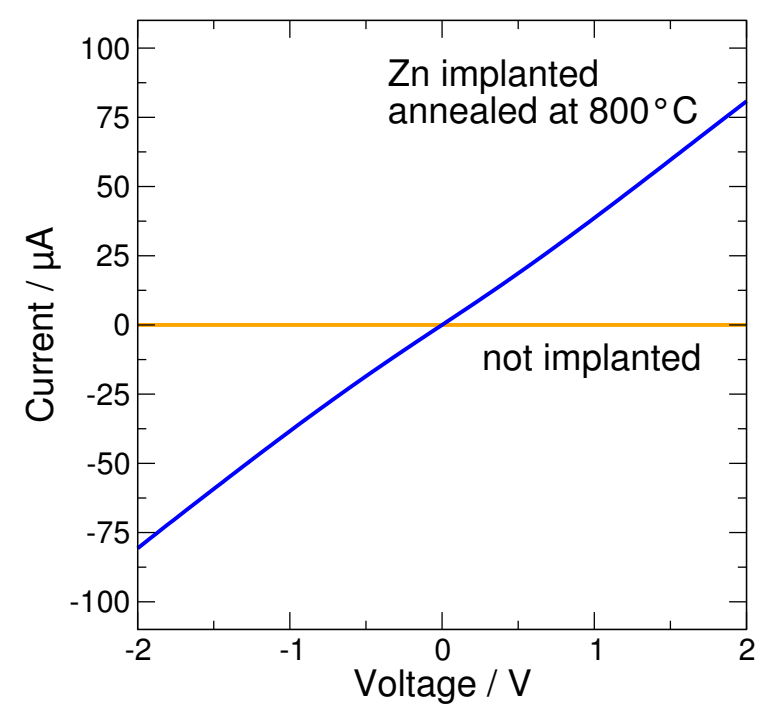

Abbildung 6.3: Strom-Spannungskennlinien von Galliumarsenid-Nanodrähten, die mit Zink implantiert wurden im Vergleich zu nichtimplantierten Nanodrähte aus dem gleichen Wachstumsprozess.

der Kristallinität eine mögliche Erklärung für diese Beobachtung. Zusätzlich können evtl. vorhandene Verunreinigungen aus dem Wachstumsprozess, wie Wasserstoff oder Kohlenstoff, bei der Temperaturbehandlung ausdiffundieren, was ebenfalls zu der beobachteten Verringerung des Widerstandes führen würde [Che92, Lat99].

Um den Kontaktwiderstand der Leiterbahnen an dem Nanodraht zu ermitteln, wurden an einem Nanodraht Kontakte mit unterschiedlichem Abstand angebracht (vgl. Abbildung 6.1 b)). In Abbildung 6.2 b) ist der Widerstand als Funktion der Länge der zugehörigen Teilabschnitte aufgetragen. Die durchgezogene Linie ist eine lineare Regression der Messwerte. Aus dieser Regressionsgerade kann durch Extrapolieren der Kontaktwiderstand $\mathrm{zu} \approx(10 \pm 3) k \Omega$ bestimmt werden.

\subsubsection{Leitfähigkeitsmessungen an Zink-implantierten Galliumarsenid-Nanodrähten}

Abbildung 6.3 zeigt die Strom-Spannungskennlinien eines mit Zink implantierten Galliumarsenid-Nanodrahtes und eines nichtimplantierten GalliumarsenidNanodrahtes. Bei einer Spannung 2 V fließt bei dem implantierten Draht ein Strom von $\approx 80 \mu \mathrm{A}$. Der resultierende Widerstand von $25 \mathrm{k} \Omega$ ist folglich um mehr als fünf 
Größenordnungen geringer als der Widerstand der nichtimplantierten und getemperten Nanodrähte aus dem selben Wachstumsprozess. Der hier ermittelte Widerstand ist in der Größenordnung des Kontaktwiderstandes, der $\mathrm{zu} \approx 8 \mathrm{k} \Omega$ angenommen wird. Die deutlich erhöhte Leitfähigkeit der vermessenen Nanodrähte weist auf eine erfolgreiche Aktivierung des implantierten Zinks als Akzeptor hin.

Um die Ladungsträgerkonzentration abzuschätzen wurde die Form des Nanodrahtes als Zylinder mit der Länge L und Radius $r_{0}$ angenommen. Der entsprechende Widerstand R ergibt sich dann zu

$$
R=\frac{L}{\sigma \pi\left(r_{0}-d\right)^{2}}
$$

Dabei bezeichnet $\sigma=q p \mu_{h}$ die Leitfähigkeit des Halbleiters. Die Dicke d des Verarmungsbereichs lässt sich durch das Oberflächenpotenzial $V_{S}$ entsprechend Gleichung 6.2, die Mobilität der Löcher $\mu_{h}$ entsprechend Gleichung 6.3 bestimmen [Sze81].

$$
\begin{gathered}
d=\sqrt{\frac{2 \epsilon_{0} \epsilon_{r} V_{S}}{q N_{A}}} \\
\mu_{h}=\frac{\mu_{0}}{1+\sqrt{\frac{N_{A}}{10^{18} \mathrm{~cm}^{-3}}}}
\end{gathered}
$$

Werden typische Werte für p-Galliumarsenid angesetzt $\left(\mu_{0}=450 \frac{\mathrm{cm}^{2}}{\mathrm{Vs}_{\mathrm{s}}}, \mathrm{V}_{\mathrm{S}}=0,45 \mathrm{~V}\right.$ und $\epsilon_{\mathrm{r}}=13,1$ [Mei78]) sowie die vermessene Nanodrahtlänge $\mathrm{L}=1 \mu \mathrm{m}$ und der gemessene Widerstand um den Kontaktwiderstand korrigiert, so ergibt sich die Konzentration der Löcher $\mathrm{zu} \mathrm{p} \approx 3,5 \cdot 10^{18} \mathrm{~cm}^{-3}$. Dieser berechnete Wert ist allerdings als Abschätzung zu verstehen, da die extrem hohe Dotierkonzentration zu einer Entartung der Dotierniveaus zu Bändern führt und so das Modell nicht mehr zutrifft [Fis69]. Außerdem kann ein Sättigungseffekt der Ladungsträgerkonzentration mit steigender Defektkonzentration im hochdotierten Halbleiter eintreten. Grund hierfür kann beispielsweise die lokale Anreicherung von Dotiersubstanzen sein [Cha07b]. Desweiteren sind bei Raumtemperatur in hochdotierten Halbleitern nur ein Bruchteil der Akzeptoren ionisiert [Fis69] und der Kontaktwiderstand nicht exakt bekannt. Entsprechend kann davon ausgegangen werden, dass die Löcherkonzentration in etwa der Konzentration der implantierten Zinkionen entspricht. Die Ladungsträgerkonzentration der undotierten Nanodrähte ist hingegen so gering, dass diese als vollständig verarmt angenommen werden können. 


\subsection{Feldeffekttransistoren basierend auf Zinkoxid- Nanodrähten}

Wie bereits in Abschnitt 4.1 beschrieben eignet sich Zinkoxid aufgrund seiner Strahlenhärte insbesondere für den Einsatz als elektronisches Bauteil in strahlenbelasteten Bereichen [Ju06]. Da eine erfolgreiche p-Dotierung von Zinkoxid noch nicht erreicht wurde, muss hier auf unipolare Bausteine wie den Feldeffekttransistor (FET) zurückgegriffen werden, der ein grundlegendes Bauelement in der Halbleiterelektronik darstellt. An einer Vielzahl von Nanodraht-basierten Feldeffekttransistoren wurde gezeigt, dass sie konventionelle CMOS-Bausteine in vielen Leistungsmerkmalen übertreffen [Jav02, Win02]. Feldeffekttransistoren eignen sich außerdem dazu Aufschluss über wesentliche elektrische Eigenschaften der verwendeten Nanodrähte zu erlangen.

In Kollaboration mit der Gruppe Tegude/ Universität Duisburg und Lu/ UCI wurden Zinkoxid-Nanodrähte, die in Göttingen und an der UCI hergestellt wurden, zu Feldeffekttransistoren prozessiert und elektrisch vermessen. In den folgenden Abschnitten wird zunächst der Aufbau und die Herstellung von NanodrahtFeldeffekttransistoren sowie die Extraktion wesentlicher elektrischer Kenngrößen beschrieben, dann der Einfluss der Oberfläche und des Durchmesser der Nanodrähte sowie der Geometrie der Basis anhand der Messergebnisse diskutiert.

\subsubsection{Herstellung der Feldeffekttransistoren}

Zur Herstellung der Feldeffekttransistoren wurden zwei unterschiedliche Prozesse verwendet: Zum einen wurden elektrische Leiterbahnen mittels optischer Lithographie an Zinkoxid-Nanodrähte angebracht. Die Nanodrähte befanden sich dabei auf einem hochdotiertem Siliziumsubstrat $\left(\mathrm{p}^{++}-\mathrm{Si}\right)$, auf dem zuvor thermisch eine $500 \mathrm{~nm}$ dicke Siliziumoxidschicht hergestellt wurde. Während die Kontakte als Emitter und Kollektor dienten, wirkte dass leitfähige Substrat als rückseitige Basis (Backgate). Zum anderen wurden die Nanodrähte auf einem isolierendem Substrat (Siliziumoxid auf einem Galliumarsenidsubstrat) dispergiert. Mittels Elektronenstahllithographie wurden ebenfalls Kontakte an deren Enden angebracht. Anschließend wurden die Nanodrähte in einem CVD-Prozess mit einer $30 \mathrm{~nm}$ dicken, isolierenden Siliziumnitridschicht $\left(\mathrm{SiN}_{x}\right)$ überzogen. In einem weiteren Lithographie- 
a)

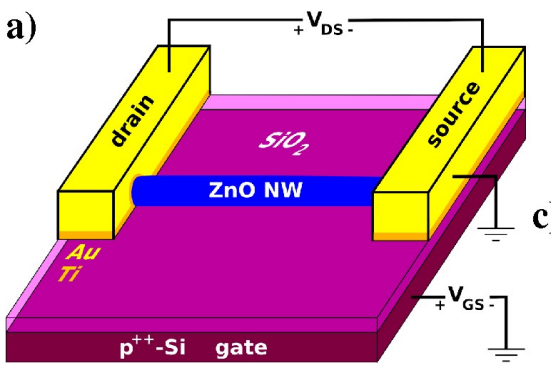

b)

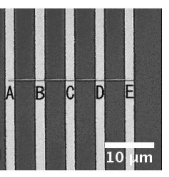

c)

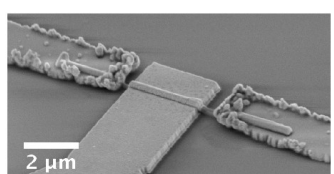

d)

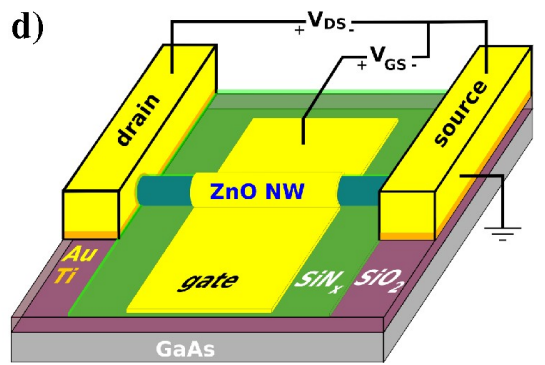

Abbildung 6.4: Aufbau der verwendeten Feldeffekttransistoren. a) Schematische Darstellung der Messgeometrie bei der Verwendung einer rückseitigen Basis. b) SEM-Aufnahme der verwendeten Messgeometrie. c) SEM-Aufnahme eines Zinkoxid-Nanodraht-Feldeffekttransistors mit frontseitiger Basis. c) Schematische Darstellung der Messgeometrie bei der Verwendung einer frontseitigen Basis.

schritt wurde zwischen den Kontakten auf die $S i N_{x}$-Schicht ein weiterer Kontakt aufgebracht, der als frontseitige Basis diente (Topgate). Die Kontakte bestehen jeweils aus einem Schichtpaket aus Titan (10 nm) und Gold (100 nm) [Ju07, Ip06]. Eine schematische Darstellungen sowie SEM-Aufnahmen der prozessierten Nanodrähte sind in Abbildung $6.4 \mathrm{zu}$ sehen.

\subsubsection{Eigenschaften der Feldeffekttransistoren}

Abbildung 6.5 zeigt Kennlinien eines Nanodraht-Feldeffekttransistors mit rückseitiger Basis (vgl. Abbildung 6.4 a)). Der verwendete Zinkoxid-Nanodraht hat einen Durchmesser von $\approx 80 \mathrm{~nm}$ und der Abstand zwischen Emitter- und Kollektorelektrode beträgt $4 \mu \mathrm{m}$. In Abbildung 6.5 a) sind Strom-Spannungskennlinien zwischen Emitter und Kollektor (Ausgangskennlinie) im linearen/ohmschen Bereich $\left(V_{D S}<V_{G S}\right)$ für Basisspannungen $\left(V_{G S}\right)$ zwischen +1 und $+10 \mathrm{~V}$ aufgetragen. In diesem ohmschen Bereich verhält sich der Feldeffekttransistor wie ein ohmscher Leiter, dessen Widerstand sich durch die Basisspannung regulieren lässt [Sze81]. Der lineare Verlauf der gemessenen Kennlinien ist ein gutes Indiz für ohmsche Kontakte an den Nanodrähten. Desweiteren ist ein Einfluss der Basisspannung auf den Kollektorstrom $\left(I_{D S}\right)$ zu beobachten, was ein Hinweis auf die Funktionstüchtigkeit des Bauelements ist. Da mit steigender Basisspannung der Kollektorstrom zunimmt, sind Elektronen Majoritätsladungsträger in dem Nanodraht. Dies ist in Übereinstimmung mit Lumineszenzuntersuchungen an diesen Nanodrähten (vgl. 

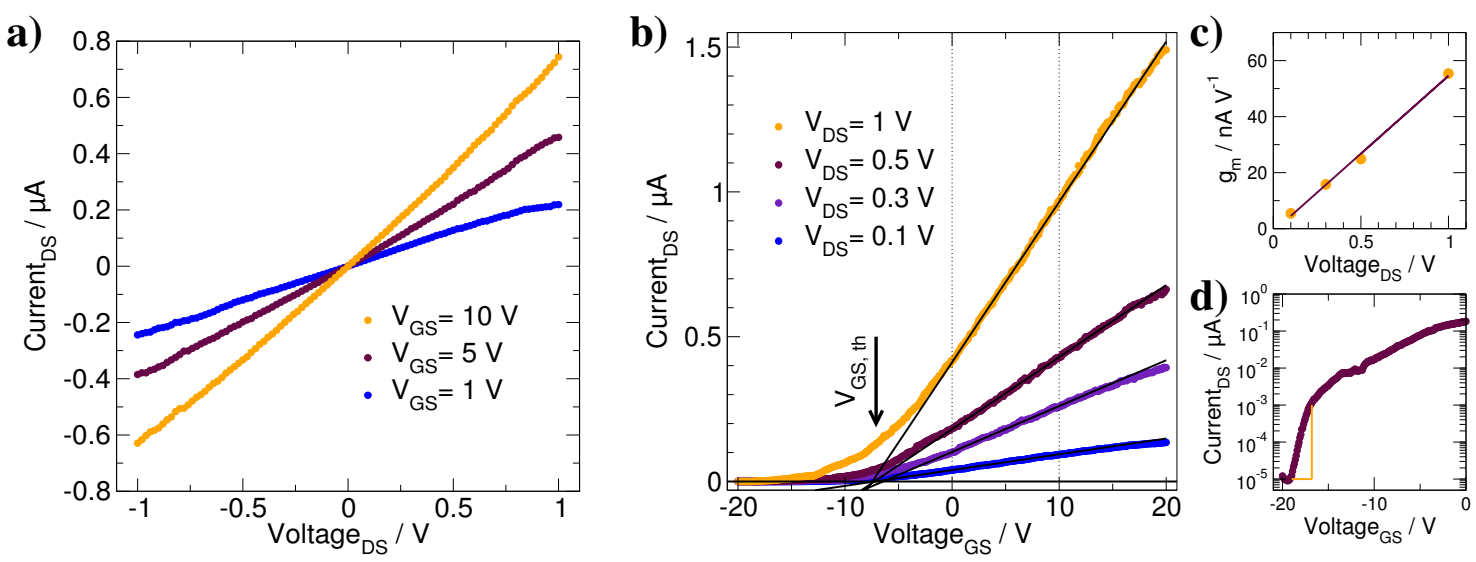

Abbildung 6.5: Kennlinien eines Zinkoxid-Nanodraht-Feldeffekttransistors in BackgateGeometrie. a) Ausgangskennlinien für unterschiedliche Basisspannungen im linearen Bereich. b) Steuerkennlinien des Feldeffekttransistors für unterschiedliche Kollektorspannungen. c) Transkonduktanz $g_{m}$ des FETs als Funktion der Kollektorspannung. d) Steuerkennlinie des Feldeffekttransistors bei $\mathrm{V}_{\mathrm{DS}}=0,5 \mathrm{~V}$ auf einer halblogarithmischen Skala.

Kapitel 3) - alle Spektren waren von donatorgebundenen Exzitonen dominiert, was auf eine entsprechend hohe Donatorkonzentration in Zinkoxid-Nanodrähten hinweist.

Abbildung 6.5 b) zeigt den Kollektorstrom als Funktion der Basisspannung $\left(I_{D S}\left(V_{G S}\right)\right.$, Steuerkennlinie) für unterschiedliche Kollektorspannungen. Die Steuerkennlinien zeigen das typische Verhalten eines n-Kanal-VerarmungstypFeldeffekttransistors (depletion-mode) [Heo04]. Bei großen negativen Basisspannungen werden die Majoritätsladungsträger (Elektronen) aufgrund elektrostatischer Abstoßung stark kanalisiert und es kommt zu einer Verarmung an freien Ladungsträgern, was sich durch einen großen Widerstand des Nanodrahtes äußert. Ab einem negativem Schwellwert $V_{G S, t h}$ steigt der Kollektorstrom nahezu linear mit der Basisspannung an. Bei einem Verarmungstyp-Feldeffekttransistor ist typischerweise auch ohne angelegte Basisspannung ein Kollektorstrom zu messen. Ursache dafür sind bei den verwendeten Zinkoxid-Nanodrähten die bei Raumtemperatur zu großem Teil ionisierten Donatoren.

Aus den Steuerkennlinien lassen sich die wichtigen Kenngrößen des Feldeffekttransistors entnehmen: Die Steilheit $g_{m}\left(=\frac{d I_{D S}}{d V_{G S}}\right.$, Transkonduktanz) der Steuerkennlinie wurde für unterschiedliche Kollektorspannungen durch Anpassen der Steuerkennli- 
nien im linearen Bereich zwischen 0 und $10 \mathrm{~V}$ ermittelt und ist in Abbildung $6.5 \mathrm{c}$ ) als Funktion der Kollektorspannung $V_{\text {DS }}$ dargestellt. Die durchgezogene Linie zeigt eine lineare Regression der Messwerte. Es ergibt sich eine Steigung von $\approx 56 \frac{\mathrm{nA}}{\mathrm{V}^{2}}$. Daraus lässt sich eine gemittelte Transkonduktanz für $V_{D S}=1 \mathrm{~V}$ ableiten, die unter Berücksichtigung der Nanodrahtabmessungen $g_{m}^{*}=\frac{\left.g_{m}\right|_{V_{D S}=1}}{2 r_{0}} \approx 0,7 \frac{\mathrm{S}}{\mathrm{m}}$ beträgt $\left(r_{0}=40 \mathrm{~nm}\right)$. Aus der Anpassung der Steilheit der Steuerkennlinien kann außerdem durch lineares Extrapolieren die Einsatzspannung des Transistors zu $\mathrm{V}_{\mathrm{GS}, \mathrm{th}} \approx(-7,1 \pm 0,2) \mathrm{V}$ bestimmt werden.

Eine weitere wichtige Kenngröße eines Transistors ist die Steilheit der Steuerkennlinie unterhalb der Einsatzspannung S (Subthresholdswing). In diesem Spannungsbereich liegt idealerweise eine vollständige Verarmung an Ladungsträgern im Nanodraht vor. Allerdings ist durch die thermische Aufweichung der Besetzungsdichte nach Boltzmann- bzw. Fermistatistik auch unterhalb der Schwellenspannung noch ein Strom zu messen, der entsprechend der Besetzungsstatistik exponentiell mit der Basisspannung abnimmt [Tsi87, Sze81]. Die Steilheit der Steuerkennlinie unterhalb von $V_{G S, t h}$ ist folglich definiert zu $S=d V_{G S} / d\left(\log \left(I_{D S}\right)\right)$. In der oben gezeigten Kennlinie beträgt $\mathrm{S}$ etwa 1,1 V/Dekade (vgl. Abbildung 6.5 d)). Da S im Idealfall nur durch die Besetzungsstatistik vorgegeben ist, sollte bei Raumtemperatur gelten $\mathrm{S}=\frac{\mathrm{k}_{\mathrm{B}} \cdot 300 \mathrm{~K}}{\mathrm{q}_{\mathrm{e}}} \ln (10) \approx 60 \mathrm{mV} /$ Dekade. Reale Bauelemente weisen allerdings durch Defektzustände an der Oberfläche [Hon03] sowie durch einen Überlapp der Basiskapazität mit der Kapazität zwischen Emitter und Kollektor [Sze81, Win02] größere Werte auf. Diese Größe ist in mehrfacher Hinsicht von großer Bedeutung für die Leistungsfähigkeit eines Feldeffekttransistors: je niedriger der Leckstrom unterhalb der Einsatzspannung des Transistors ist, desto geringer sind auch die thermischen Verluste des Transistors. Bei einer hohen Integrationsdichte von Transistoren ist die Wärmeproduktion eine entscheidende Größe. Die zweite Bedeutung kommt S bei hochfrequenter Schaltung des Transistors bei niedrigen Leistungen und Strömen zu - hier bestimmt S die Schaltzeit des Transistors [Sze81]. Typische Werte für S von Hochleistungstransistoren auf Silizium- bzw. Kohlenstoff-Nanodrahtbasis betragen 70 - $100 \mathrm{mV} /$ Dekade und sind damit deutlich besser als dieser Zinkoxid-NanodrahtFET [Win02, Jav02, Rau99].

Aus den ermittelten Kenngrößen lassen sich weitere Eigenschaften der Nanodrähte ableiten, insbesondere die Ladungsträgermobilität und -konzentration. Entspre- 
chend den Berechnungen von Shockley gilt für den Ausgangsstrom $I_{D S}$ eines Feldeffekttransistors mit langem Kanal folgende Näherung [Sho52]:

$$
I_{D S}=\frac{\mu \cdot C_{G}}{L_{G}^{2}}\left[\left(V_{G S}-V_{G S, t h}\right) V_{D S}-\frac{1}{2} V_{D S}^{2}\right] .
$$

Dabei bezeichnet $\mu$ die Ladungsträgermobilität und $L_{G}$ die Basislänge. Die Kapazität der Basis $C_{G}$ lässt sich mit Gleichung 6.5 unter der Annahme folgender Geometrie berechnen [Ju07]: eingebettet in ein Dielektrikum $\left(\epsilon_{r}\right)$ befindet sich ein zylinderförmiger Nanodraht (Länge $L=L_{G}$, Radius $r_{0}$ ) im Abstand $h$ von einer unendlich ausgedehnten Fläche. Die Basiskapazität ergibt sich damit zu:

$$
C_{G}=\frac{2 \pi \epsilon_{r} \epsilon_{0} L_{G}}{\operatorname{acosh}\left(1+\frac{h}{r_{0}}\right)} .
$$

Mit den Eigenschaften des verwendeten Feldeffekttransistors $\left(\epsilon_{r}\left(\mathrm{SiO}_{2}\right)=3,9\right.$, $L_{G}=4 \mu \mathrm{m}, \mathrm{h}=500 \mathrm{~nm}, r_{0}=40 \mathrm{~nm}$ ) berechnet sich die Basiskapazität $\mathrm{zu} \approx 0,20 \mathrm{fF}$. Es sei an dieser Stelle darauf hingewiesen, dass die Berechnung der Kapazität zwar exakt ist, die Geometrie allerdings nur eine Annahme. Insbesondere ist der Nanodraht nicht wie im Modell in ein Dielektrikum eingebettet. Entsprechend sind die im Folgenden daraus abgeleiteten Größen als Abschätzung zu verstehen.

Durch Ableitung von Gleichung 6.4 nach der Basisspannung lässt sich ein Ausdruck für die Ladungsträgermobilität finden [Sze81]:

$$
\begin{aligned}
g_{m} & =\frac{d I_{D S}}{d V_{G S}}=\frac{\mu \cdot C_{G}}{L_{G}^{2}} V_{D S} \\
\Rightarrow \mu & =\frac{L_{G}^{2}}{C_{G}} \cdot \frac{g_{m}}{V_{D S}} .
\end{aligned}
$$

Mit der oben bestimmten Transkonduktanz bei $V_{D S}=1 \mathrm{~V}$ ergibt sich eine Ladungsträgermobilität von $\approx 45 \frac{\mathrm{cm}^{2}}{\mathrm{Vs}}$.

Auf ähnliche Weise lässt sich auch die Ladungsträgerkonzentration n abschätzen. Da bei der Schwellenspannung $\mathrm{V}_{\mathrm{GS} \text {,th }}$ gerade eine Ladungsträgerverarmung einsetzt, lässt sich die Ladungsträgerkonzentration im Nanodraht mit Hilfe der Kapazität der Basis wie folgt bestimmen [Mar98, Sze81]:

$$
\begin{aligned}
Q & =C_{G} \cdot V_{G S, t h} \\
\Rightarrow n & =\frac{C_{G} \cdot V_{G S, t h}}{\pi r_{0}^{2} L_{G} q_{e}} .
\end{aligned}
$$


Unter Annahme der oben gegebenen Parameter ergibt sich eine Ladungsträgerkonzentration von $\approx 5 \cdot 10^{17} \mathrm{~cm}^{-3}$. Alternativ lässt sich die Ladungsträgerkonzentration auch aus der Leitfähigkeit des Drahtes abschätzen.

$$
\sigma=n \cdot q_{e} \cdot \mu
$$

Die Leitfähigkeit $\sigma$ lässt sich aus den Achsenabschnitten der Steuerkennlinien und der Geometrie des Drahtes zu $\approx 305 \frac{S}{m}$ extrahieren. Mit dem zuvor bestimmten Wert für die Mobilität ergibt sich im Rahmen des Fehlers in etwa der gleiche Wert für die Ladungsträgerkonzentration. Sowohl die Ladungsträgerkonzentration als auch die -mobilität sind vergleichbar mit Werten, die in der Literatur für ähnliche Messanordnungen berichtet wurden: $1 \cdot 10^{17}-1 \cdot 10^{18} \mathrm{~cm}^{-3}$ [Hon03], $2 \cdot 10^{17} \mathrm{~cm}^{-3}, 50-200 \frac{\mathrm{cm}^{2}}{V s}$ [Ju05].

\subsubsection{Einfluss des Nanodrahtdurchmessers}

In optischen Untersuchungen an Zinkoxid-Nanodrähten mit geringem Durchmesser konnte vor allem die Auswirkung des erhöhten Oberflächen-zu-Volumenverhältnises beobachtet werden. Auch auf die elektrische Leitfähigkeit hat die ZinkoxidOberfläche einen starken Einfluss [Hon03]. Insbesondere durch eine Wechselwirkung zwischen Sauerstoffleerstellen an der Oberfläche und Adsorbaten kommt es zu einer starken Veränderung des Oberflächenpotenzials und der Leitfähigkeit - entsprechend findet Zinkoxid neben Zinnoxid breite Anwendung in der Gassensorik [Era04, Fan04]. Außerdem wurde eine deutliche Verbesserung der Leistungsmerkmale von Zinkoxid-Nanodraht-Feldeffekttransistoren nach einer Oberflächenbehandlung mit Ozon beobachtet [Ju05]. Um den Einfluss des Oberflächenzu-Volumenverhältnises auf die Leitfähigkeit zu überprüfen, wurden analog zu dem oben beschriebenen Prozess Nanodrähte mit deutlich geringerem Durchmesser von 10 bis $20 \mathrm{~nm}$ zu Feldeffekttransistoren prozessiert [Cha07a].

Abbildung 6.6 zeigt Ausgangskennlinien eines Feldeffekttransistors, bei dem der verwendete Nanodraht einen Durchmesser von $18 \mathrm{~nm}$ hat. Die Kennlinien sind ebenso wie die oben diskutierten Kennlinien linear, was auf gute ohmsche Kontakte an den Nanodrähten hinweist. Außerdem weist die Linearität der Kennlinien auf den Betrieb im ohmschen Bereich des Transistors hin, was aufgrund der Spannungsverhältnisse $\left(V_{D S} \ll V_{G S}\right)$ auch zu erwarten war. Im Gegensatz zu den Nano- 


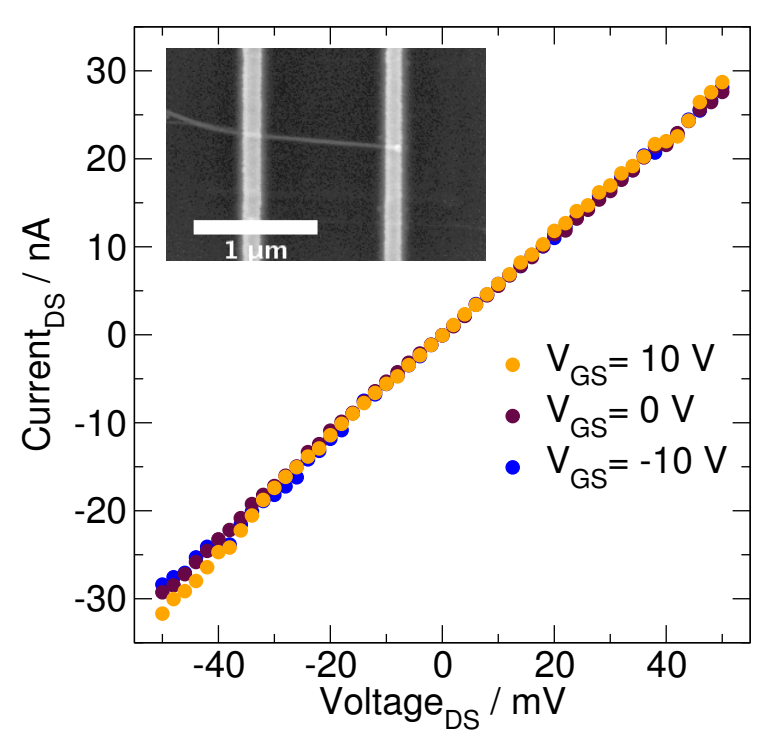

Abbildung 6.6: Ausgangskennlinien eins Zinkoxid-Nanodraht-Feldeffekttransistors bei unterschiedlichen Basisspannungen. Im Einsatz ist eine SEM-Aufnahme der untersuchten Probe zu sehen.

drähten mit größerem Durchmesser zeigen die Kennlinien in Abbildung 6.6 nur eine sehr geringe Abhängigkeit von der angelegten Basisspannung. Aus der Steigung der Strom-Spannungskennlinien kann die Leitfähigkeit des Nanodrahtes zu $\sigma \approx 2300 \frac{\mathrm{S}}{\mathrm{m}}$ bestimmt werden $\left(r_{0}=9 \mathrm{~nm}, L_{G}=1 \mu \mathrm{m}\right)$. Aufgrund der geringen Abhängigkeit von der Basisspannung kann die Transkonduktanz lediglich aus den Ausgangskennlinien zu $g_{m}^{*}=0,3 \frac{\mathrm{S}}{\mathrm{m}}$ abgeschätzt werden. Mit der Basiskapazität des verwendeten Transistors von $\approx 35 \mathrm{aF}$ und der Basislänge von $1 \mu \mathrm{m}$ (vgl. Einsatz in Abbildung 6.6) lässt sich mit Gleichung 6.6 die Ladungsträgermobilität $\mathrm{zu} \mu=1,5 \frac{\mathrm{cm}^{2}}{\mathrm{Vs}}$ berechnen. Ein Vergleich der Werte für die Leitfähigkeit und Mobilität mit den Werten, die für Nanodrähte mit größerem Durchmesser ermittelt wurden, zeigt, dass die Leitfähigkeit der dünnen Nanodrähte um eine Größenordnung höher ist, während die Mobilität um mehr als eine Größenordnung geringer ist. Entsprechend Gleichung 6.8 ist die Ladungsträgerkonzentration in den dünnen Nanodrähten mit $\approx 10^{20} \mathrm{~cm}^{-3}$ um mehr als zwei Größenordnungen höher als bei Nanodrähten größeren Durchmessers.

Die dünnen Zinkoxid-Nanodrähte mit extrem hoher Ladungsträgerkonzentration, geringer Ladungsträgermobilität und sehr geringem Einfluss der Basisspannung 
zeigen nahezu metallischen Charakter. Dieses Verhalten wird hauptsächlich auf zusätzliche Ladungsträger an der Oberfläche der Nanodrähte zurückgeführt. Die zusätzlichen Ladungsträger an der Oberfläche führen dann zu einer geringeren Bandverbiegung [Hon03] was wiederum gute ohmsche Kontakte ermöglicht.

Im Vergleich zu den Lumineszenzuntersuchungen an Zinkoxid-Nanodrähten mit unterschiedlichem Durchmesser zeigt sich auch in den hier vorgestellten elektrischen Messungen ein deutlicher Einfluss von Oberflächenzuständen. Interessanterweise tritt die starke Veränderung der Eigenschaften bei vergleichbaren Durchmessern auf. Eine direkte Korrelation der verursachenden Defekte ist allerdings schwierig zu treffen.

\subsubsection{Passivierung der Nanodrahtoberfläche}

Um den Einfluss der Oberfläche zu reduzieren und die Leistungsfähigkeit der Transistoren zu erhöhen wurden Nanodraht-Feldeffekttransistoren hergestellt, deren Oberfläche passiviert ist [Cha06]. Eine Verbesserung der Leistungsmerkmale durch organische Beschichtungen wurde bereits in der Literatur an Silizium-Nanodrähten und Zinkoxid-Nanodrähten gezeigt [Cui03, Par04a, Ju05], allerdings ist dabei die Langzeitbeständigkeit unklar. Hier wurde die Nanodrähte zur Passivierung ins Vakuum gebracht um Oberflächenadsorbate zu entfernen. Anschließend wurden die Nanodrähte in ein Schichtpaket aus $100 \mathrm{~nm} \mathrm{SiO}_{2}$ und $50 \mathrm{~nm} \operatorname{SiN}_{x}$ eingebettet.

Abbildung 6.7 a) zeigt Strom-Spannungskennlinien zwischen Emitter und Kollektor bei unterschiedlichen Basisspannungen im linearen Bereich. Auch hier werden lineare Kennlinien beobachtet, was auf einen ohmschen Kontakt an den Nanodrähten hinweist. Die Abhängigkeit von der Basisspannung bestätigt wiederum die n-typ Leitfähigkeit der Nanodrähte. Die Steuerkennlinien der passivierten Feldeffekttransistoren in Abbildung 6.7 b) zeigen ein wesentlich verbessertes Schaltverhalten. Der Transistor lässt sich bei deutlich geringeren Kollektorspannungen schalten und zeigt ein ausgeprägtes Sättigungsverhalten. Die Steilheit unterhalb der Einsatzspannung von $500 \mathrm{mV} /$ Dekade konnte durch die Oberflächenpassivierung deutlich verbessert werden (vgl. Abbildung $6.7 \mathrm{~d}$ )). In der halblogarithmischen Darstellung der Kennlinie ist bei großen negativen Basisspannungen wieder ein Anstieg des Kollektorstroms zu beobachten. Dies ist vermutlich auf eine einsetzende Löcherleitung 

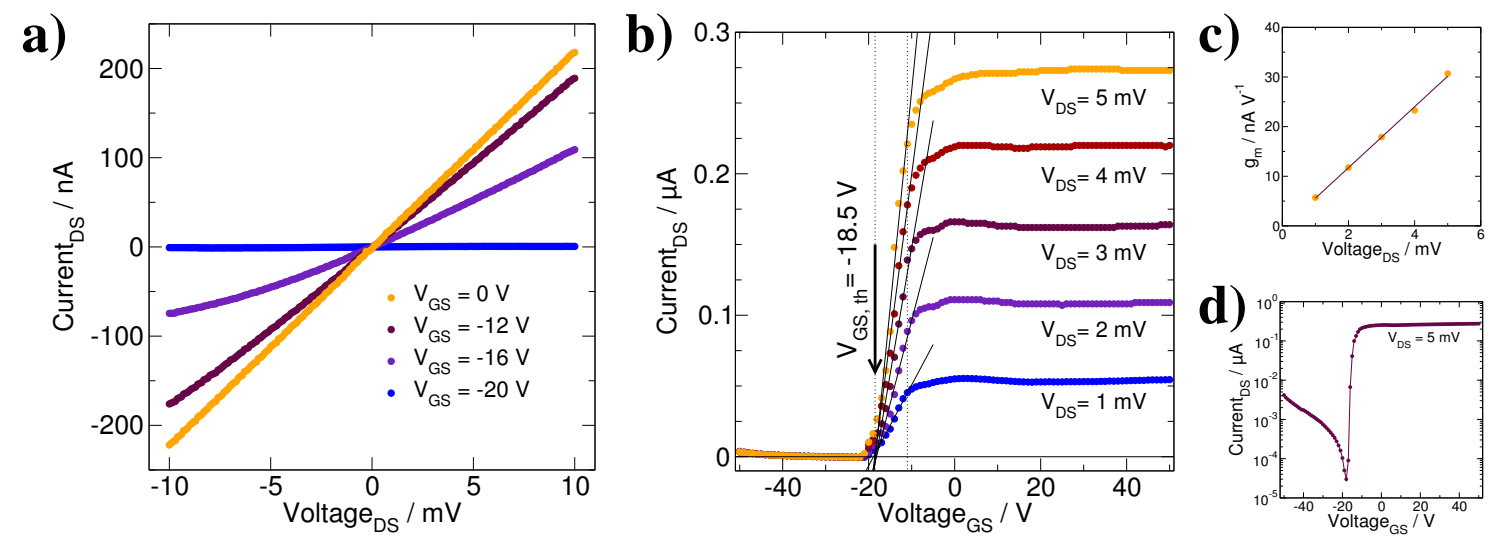

Abbildung 6.7: Kennlinien eines Zinkoxid-Nanodraht-Feldeffekttransistors mit Oberflächenpassivierung in Backgate-Geometrie. a) Ausgangskennlinien für unterschiedliche Basisspannungen im linearen Bereich. b) Steuerkennlinien des Feldeffekttransistors für unterschiedliche Kollektorspannungen. c) Transkonduktanz $g_{m}$ des FETs als Funktion der Kollektorspannung. d) Steuerkennlinie des Feldeffekttransistors bei $\mathrm{V}_{\mathrm{DS}}=5 \mathrm{mV}$ auf einer halblogarithmischen Skala.

zurückzuführen.

Aus der Anpassung der Transkonduktanz $g_{m}$ ergibt sich eine relativ große Einsatzspannung von $V_{G S, t h}=-18,5 \pm 0,1 \mathrm{~V}$. Der Durchmesser des Nanodrahtes wurde zu $\approx 90 \mathrm{~nm}$ bestimmt, woraus sich mit Gleichung 6.5 eine Basiskapazität von $\approx 0,21 \mathrm{fF}$ berechnet. Die Ladungsträgerkonzentration lässt sich mit Gleichung $6.7 \mathrm{zu}$ $1 \cdot 10^{18} \mathrm{~cm}^{-3}$ bestimmen und ist damit mehr als doppelt so hoch wie in dem nichtpassivierten Nanodraht. In Abbildung 6.7 c) ist die Transkonduktanz als Funktion der Kollektorspannung aufgetragen. Auch bei diesem Feldeffekttransistor ergibt sich ein guter linearer Zusammenhang. Die Steigung lässt sich in einer linearen Regression $\mathrm{zu} \approx 6,2 \cdot 10^{-6} \frac{A}{V^{2}}$ bestimmen. Auf den Durchmesser des Nanodrahtes normiert ergibt sich eine Transkonduktanz von $g_{m}^{*}=68 \mathrm{~S} / \mathrm{m}$, die zwei Größenordnungen höher ist als bei dem nichtpassivierten Nanodraht. Entsprechend ist auch die Ladungsträgermobilität, die sich mit Gleichung $6.3 \mathrm{zu} \approx 4800 \frac{\mathrm{cm}^{2}}{V S}$ berechnet, um mehr als zwei Größenordnungen höher als bei den nichtpassivierten Feldeffekttransistoren.

Abgesehen von der großen negativen Einsatzspannung und dem recht großen Subthresholdswing zeigt der Zinkoxid-Nanodraht-Transistor hervorragende Eigenschaften. 

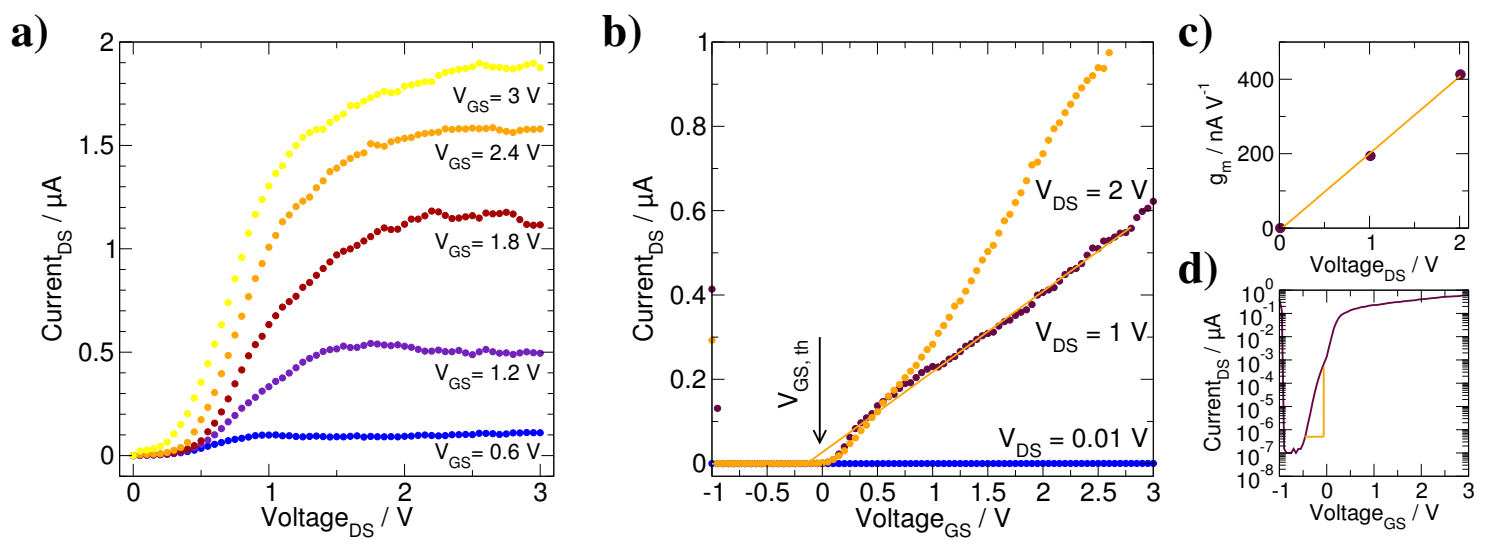

\begin{abstract}
Abbildung 6.8: Kennlinien eines Zinkoxid-Nanodraht-Feldeffekttransistors mit Oberflächenpassivierung in Topgate-Geometrie. a) Ausgangskennlinien für unterschiedliche Basisspannungen zeigen ein typisches Sättigungsverhalten (pinch-off). b) Steuerkennlinien des Feldeffekttransistors für unterschiedliche Kollektorspannungen. c) Transkonduktanz $g_{m}$ des FETs als Funktion der Kollektorspannung. d) Steuerkennlinie des Feldeffekttransistors bei $V_{D S}=1 \mathrm{~V}$ auf einer halblogarithmischen Skala.
\end{abstract}

\title{
6.2.5 Topgate-Feldeffekttransistor
}

Um die Einsatzspannung weiter zu reduzieren ist ein besserer elektrostatischer Einfluss auf die Ladungsträger im Nanodraht notwendig. Dies lässt sich insbesondere erreichen, indem die Dicke des Dielektrikums verkleinert und die Einhüllung des Nanodrahtes durch die Basis vergrößert wird [Win02]. Realisiert wird dies, indem die Basiselektrode in Topgate-Geometrie aufgebracht wird (vgl. Abbildung 6.4 b)). Neben einer geringen Steuerspannung hat die Topgate-Geometrie eine Vielzahl weiterer Vorteile: bei mehreren Feldeffekttransistoren auf einem Substrat kann jeder separat angesteuert werden. Die Geometrie eignet sich für Hochfrequenzanwendungen, was für die Backgate-Variante aufgrund des großen Überlapps mit der KollektorEmitterkapazität nicht in Frage kommt. Zusätzlich ist der Nanodraht in dieser Geometrie grundsätzlich in ein Dielektrikum eingebettet. Damit ist die Kapazität besser definiert und die Drahtoberfläche passiviert [Win02].

Abbildung 6.8 a) zeigt die Ausgangskennlinien eines Zinkoxid-NanodrahtFeldeffekttransistors mit frontseitiger Basis. Die Kennlinien zeigen bei großen Kollektorspannungen $\left(V_{D S} \gtrsim V_{G S}\right)$ ein typisches Sättigungsverhalten. Durch den Spannungsabfall zwischen Emitter und Kollektor über die Basislänge kommt es zu ei- 
nem zusätzlichen elektrischen Feld, dass bei hohen Kollektorspannungen zu einer Einschnürung des leitfähigen Kanals im Nanodraht führt (pinch-off) [Sze81]. Bei niedrigen Kollektorspannungen zeigen die Kennlinien einen S-förmigen Verlauf, was vermutlich auf die Kontakte zu den Zinkoxid-Nanodrähten zurückzuführen ist. Die Kennlinien zeigen ein deutliches n-Kanal-Verhalten: der Kollektorstrom steigt mit zunehmend positiver Basisspannung an.

In Abbildung 6.8 b) sind Steuerkennlinien des Feldeffekttransistors für unterschiedliche Kollektorspannungen dargestellt. Die Transkonduktanz wurde durch lineare Regression der Steuerkennlinien im linearen Bereich ermittelt und ist in Abbildung 6.8 c) als Funktion der Kollektorspannung dargestellt. Auch hier ergibt sich wie erwartet ein linearer Zusammenhang, der normiert auf den Nanodrahtdurchmesser zu einer Transkonduktanz von $g_{m}^{*}=1,9 \frac{S}{m}$ führt. Die Einsatzspannung des Transistors lässt sich durch Extrapolieren der Transkonduktanz zu $V_{G S, t h}=(-0,02 \pm 0,02) \mathrm{V}$ ermitteln und ist deutlich geringer als bei den Feldeffekttransistoren mit rückseitiger Basis.

Zur Berechnung der Ladungsträgermobilität und -konzentration muss zunächst die Basiskapazität bestimmt werden. Diese lässt sich aufgrund der veränderten Geometrie mit dem oben beschriebenen Modell nicht berechnen. Die Kapazität für diese Geometrie ergibt sich wie folgt [Pro07]:

$$
C_{G}=\frac{2 \pi \epsilon_{r} \epsilon_{0} L_{G}}{\ln \left(\alpha \frac{h+r_{0}}{r_{0}}\right)} .
$$

Dabei bezeichnet $\alpha$ einen Faktor, der als Maß für die Ummantelung des Nanodrahtes durch die Basis aufgefasst werden kann. Für eine vollständige Ummantelung ist $\alpha=1$; hier wird $\alpha$ zu 1,55 angenommen [Pro07]. Mit den entsprechenden Eigenschaften und Abmaßen des vorliegenden Feldeffekttransistors ergibt sich eine Kapazität von $\approx 1,04 \mathrm{fF}\left(L_{G}=3 \mu \mathrm{m}, r_{0}=50 \mathrm{~nm}, h=30 \mathrm{~nm}, \epsilon_{r}=7,5\right)$. Die Ladungsträgermobilität und Konzentration ergibt sich dann aus Gleichung 6.6 und $6.7 \mathrm{zu} n=6 \cdot 10^{15} \frac{1}{\mathrm{~cm}^{3}}$ und $\mu=17 \frac{\mathrm{cm}^{2}}{V s}$. Dass die ermittelten Werte für die Ladungsträgermobilität deutlich geringer sind als bei dem passivierten Feldeffekttransistoren mit rückseitiger Basis ist vermutlich auch auf einen größeren Kontaktwiderstand zurückzuführen. Die geringere Ladungsträgerkonzentration lässt sich vermutlich auf den abweichenden Herstellungsprozess zurückführen: die hier verwendeten 


\begin{tabular}{lllllll}
\hline \hline & & & & & & \\
& $\begin{array}{l}\text { ZnO FET } \\
\text { not pass. }\end{array}$ & $\begin{array}{l}\text { ZnO FET } \\
\text { ultrathin }\end{array}$ & $\begin{array}{l}\text { ZnO FET } \\
\text { passivated }\end{array}$ & $\begin{array}{l}\text { ZnO FET } \\
\text { topgate }\end{array}$ & $\begin{array}{l}\text { CNT FET } \\
\text { Ref. [Jav02] }\end{array}$ & $\begin{array}{l}\text { ZnO FET. [Ju07] } \\
\text { Ref. }\end{array}$ \\
\hline$L_{g}(\mu \mathrm{m})$ & 4 & 1 & 4 & 3 & 2 & 2 \\
$h(\mathrm{~nm})$ & 500 & 500 & 500 & 30 & 8 & 60 \\
$C_{G}(\mathrm{fF})$ & 0,20 & 0,035 & 0,21 & 1,04 & 0,46 & $0,25^{*}$ \\
$V_{G s, t h}(\mathrm{~V})$ & $-7,1$ & - & $-18,5$ & $-0,02$ & +1 & $+0,2$ \\
$g_{m}^{*}\left(\frac{S}{m}\right)$ & 0,69 & 0,28 & 68 & 1,9 & 3000 & 125 \\
$\mu\left(\frac{c m^{2}}{V S}\right)$ & 45 & 1,5 & 4800 & 17 & 3000 & 1175 \\
$n\left(\frac{1}{c m^{-3}}\right)$ & $5 \cdot 10^{17}$ & $1 \cdot 10^{20}$ & $1 \cdot 10^{18}$ & $6 \cdot 10^{15}$ & $5 \cdot 10^{20 *}$ & $1,4 \cdot 10^{16 *}$ \\
$\sigma\left(\frac{S}{m}\right)$ & 305 & 2300 & 34500 & 2 & $2 \cdot 10^{7 *}$ & $530^{*}$ \\
$S\left(\frac{m V}{\text { Dekade }}\right)$ & 1100 & - & 550 & 130 & 70 & 130 \\
\hline
\end{tabular}

Tabelle 6.1: Zusammenfassung der Kenngrößen untersuchter Zinkoxid-NanodrahtFeldeffekttransistoren im Vergleich mit ausgewählten Beispielen aus der Literatur. Die mit * gekennzeichneten Werte wurden nicht explizit in den Referenzen genannt, sondern aus den gegebenen Werten berechnet.

Nanodrähte wurden in Göttingen bei einer höheren Temperatur und ohne Zugabe von Kohlenstoff hergestellt, was zu einer geringeren Donator- und damit auch Ladungsträgerkonzentration in den Nanodrähten führen kann.

Abbildung $6.8 \mathrm{~d}$ ) zeigt die Steuerkennlinie für $V_{D S}=1 \mathrm{~V}$ auf einer logarithmischen Skala. Hier lässt sich der Subthresholdswing $\mathrm{zu} \approx 130 \frac{\mathrm{mV}}{\text { Dekade }}$ ablesen, was eine deutliche Verbesserung gegenüber dem Feldeffekttransistor mit rückseitiger Basis ist. Dieser Wert liegt im Bereich technisch relevanter Bauelemente [Win02].

Die Ladungsträgermobilität und der Subthresholdswing können weiter verbessert werden, indem eine möglichst vollständige Basisummantelung mit Isolator und Metall gewählt wird und indem die Oberfläche des Nanodrahtes vor der Ummantelung gezielt passiviert wird. Beide Prozesse werden in der Literatur behandelt. Eine perfekte Ummantelung wird insbesondere durch einen ALD-Prozess (atomic layer deposition) erreicht [Jav02], eine effektive Oberflächenpassivierung kann durch die Behandlung mit Ozon erreicht werden [Ju07].

Ein Vergleich der Kenngrößen mit Werten, die für hochwertige Feldeffekttransistoren auf Kohlenstoffnanodrahtbasis (CNT) bzw. Zinkoxid-Basis erreicht wurde ist in Tabelle 6.2.5 aufgelistet. Der Vergleich zeigt, dass sowohl der Topgate-Transistor als auch der passivierte Backgate-Transistor sehr gute Leistungsmerkmale zeigen. 


\subsection{Nanodraht-Leuchtdioden}

Zur Funktionalisierung von Nanodrähten als elektronisches oder optoelektronisches Bauteil ist das Anbringen von elektrischen Kontakten an die Nanodrähte notwendig. Obgleich eine Vielzahl von Methoden entwickelt wurde, um Nanodrähte ausgerichtet und an zuvor bestimmten Ort auf einem Substrat zu synthetisieren [Fan06] bzw. um diese nach der Synthese auszurichten [Dua01b], werden zur elektrischen Kontaktierung meist individuelle Nanodrähte ausgewählt und an deren Enden mittels optischer Lithographie oder Elektronenstrahllithographie Kontaktflächen definiert, so wie es in den beiden Abschnitten zuvor erläutert wurde. Diese Methode kann aufgrund ihrer Aufwendigkeit nicht zu technisch relevanten Bauelementen führen. Die hier vorgestellte Methode, die in Zusammenarbeit mit M. Zimmler aus der Gruppe von Prof. Dr. F. Capasso an der Harvard University entstanden ist, führt in wenigen Prozessschritten zu einer Vielzahl von Zinkoxid-Nanodraht-Leuchtdioden, die bei Raumtemperatur im UV-Wellenlängenbereich Licht emittierten und deren Kennlinien ein gleichrichtendes Verhalten zeigen [Zim08].

Die typische Methode um Leuchtdioden herzustellen besteht darin, einen pn-Übergang im Halbleiter zu erzeugen. Dies ist bei Zinkoxid-Nanodrähten in zweifacher Hinsicht schwierig: zum einen gestaltet sich bereits die p-Dotierung von Zinkoxid-Volumenkristallen als außerordentlich schwierig (vgl. Abschnitt 3.7, [Tsu05]). Zusätzlich unterliegt die gezielte Dotierung von Nanodrähten im Allgemeinen einer besonderen Schwierigkeit (vgl. Abschnitt 6.1 und 2.1). Entsprechend wurde hier eine alternative Geometrie gewählt: die Erzeugung eines Heteroübergangs zwischen einem p-Siliziumsubstrat und den n-Zinkoxid-Nanodrähten. In der Literatur wurde bereits gezeigt, dass ein solcher p-n-Heteroübergang zu einem gleichrichtenden Verhalten und unter besonderen Voraussetzungen auch zur Lichtemission führt [Dua03, Bao06, Zim07b, Zim07a, Mot07]. Die Schwierigkeit in dieser Geometrie besteht darin, Kontakte auf den Nanodrähten anzubringen ohne dabei auch das Substrat zu kontaktieren [Bao06]. Um dies zu erreichen ist eine isolierende Zwischenschicht notwendig, die das Substrat bedeckt und in der die Nanodrähte eingebettet werden - allerdings muss dabei die Oberseite der Nanodrähte unbedeckt bleiben. Während diese Struktur in der Literatur größtenteils durch Elektronenstrahllithographie erreicht wurde, beruht der hier vorgestellte Prozess auf der planarisierenden 
a)

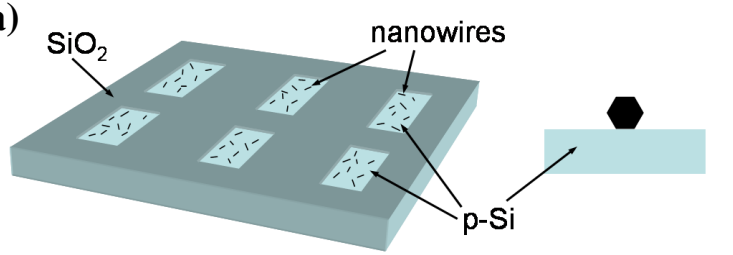

c)

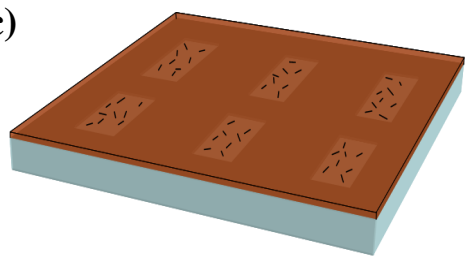

b)
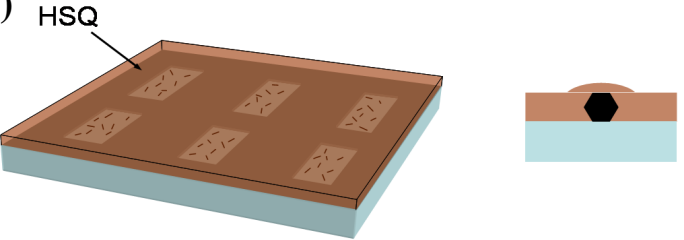

d)

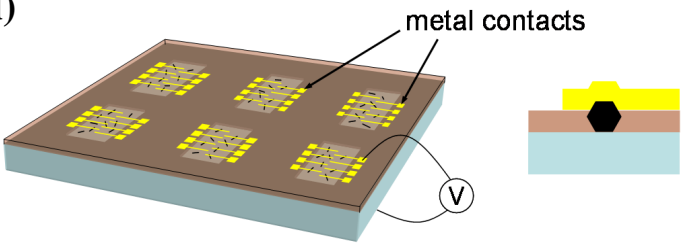

Abbildung 6.9: Schematische Darstellung der Prozessschritte zur Herstellung von NanodrahtLeuchtdioden. a) Deposition von Nanodrähten auf einem vorstrukturierten Substrat. b) Aufschleudern des planarisierenden HSQs. c) Freilegen der Nanodrahtoberfläche durch reaktives Ionenätzen. d) Aufbringen periodischer Titan-Gold-Kontakte nach einer Strukturierung mittels Photolithographie.

Eigenschaft der Substanz HSQ (hydrogen silsequioxane) - ein Resist, der durch eine Temperaturbehandlung in Siliziumoxid umgewandelt werden kann [Lio98].

\subsubsection{Präparation der Proben}

In Abbildung 6.9 ist schematisch der verwendete Prozess zur Herstellung von Zinkoxid-Nanodraht-Leuchtdioden nachgezeichnet. Ausgangspunkt ist ein hochdotiertes p-Siliziumsubstrat $\left(\approx 10^{19} \mathrm{~cm}^{-3}\right)$ auf dem thermisch eine $200 \mathrm{~nm}$ dicke Oxidschicht hergestellt wurde. Mittels Photolithographie und gepufferter Flusssäure wurden Öffnungen von $\approx 400 \times 2100 \mu \mathrm{m}$ in die Oxidschicht bis auf das p-Silizium geätzt. In einem PECVD-Prozess wurde das vorbereitete Substrat mit einer $\approx 7 \mathrm{~nm}$ dicken $\mathrm{SiO}_{2}$-Schicht übezogen. Anschließend wurden die n-Zinkoxid-Nanodrähte, die in Göttingen hergestellt wurden, auf das vorbereitete Substrat übertragen, indem das Wachstumssubstrat mit der Substratoberfläche in Kontakt gebracht wurde (vgl. Abschnitt 2.2.2). Auf die präparierten Proben wurde im zweiten Schritt HSQ (FOx-12, Dow Corning) mit einer Schichtdicke von $\approx 80 \mathrm{~nm}$ aufgeschleudert (vgl. Abbildung $6.9 \mathrm{~b})$ ) und anschließend thermisch gehärtet $\left(1 \mathrm{~min}\right.$ bei $100{ }^{\circ} \mathrm{C}$, dann $1 \mathrm{~h}$ bei $350{ }^{\circ} \mathrm{C}$ ). Durch die planarisierende Eigenschaft des HSQs ist die Schichtdicke auf den Nanodrähten deutlich geringer als auf dem Substrat. Um die Oberfläche 

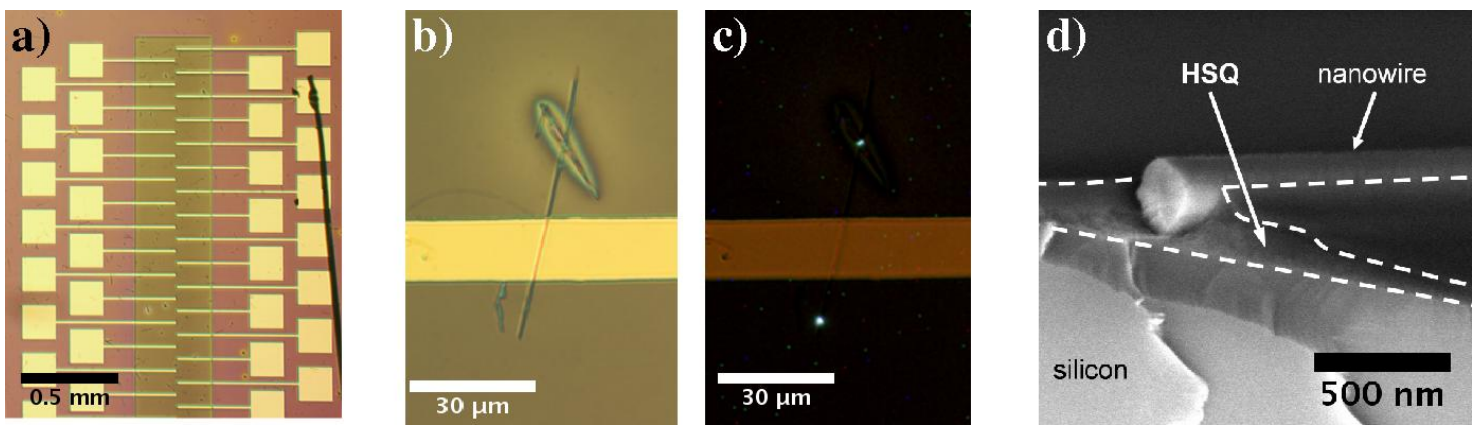

Abbildung 6.10: Licht- und elektronenmikroskopische Aufnahmen der prozessierten Nanodrähte. a) Lichtmikroskopische Aufnahme eines Messfeldes. b) Zinkoxid-Nanodraht unter einer Leiterbahn im Messfeld. c) Der gleiche Nanodraht bei abgedunkelter Beleuchtung und in Flussrichtung angelegter Spannung zwischen der Leiterbahn und dem Substrat. d) SEM-Aufnahme eines prozessierten Nanodrahtes unter flachem Winkel.

der Nanodrähte freizulegen, wurde die isolierende HSQ-Schicht mittels reaktiven Ionenätzens (RIE, $35 \mathrm{sec}, \mathrm{CF}_{4}$ at $5 \mathrm{sccm}, \mathrm{H}_{2}$ at $4 \mathrm{sccm}, 10 \mathrm{~m}$ Torr, $150 \mathrm{~W}$ Mikrowellenleistung und $50 \mathrm{~W}$ RF-Leistung) isotrop gedünnt (vgl. Abbildung 6.9c)). Abbildung 6.10 d) zeigt eine SEM-Aufnahme eines prozessierten Zinkoxid-Nanodrahtes auf dem Substrat nach dem RIE-Prozess. Es ist zu erkennen, dass die Oberseite des Nanodrahtes bereits freigelegt, während der übrige Nanodraht im HSQ eingebettet ist. Im letzten Schritt (vgl. Abbildung 6.9d)) werden periodische Strukturen von Leiterbahnen auf die Probe aufgebracht. Dazu wurden die Kontaktfelder mit Hilfe von Photolithographie definiert und thermisch ein Titan-Gold-Schichtpaket $(10 \mathrm{~nm} / 100 \mathrm{~nm})$ aufgebracht [Ip06]. Ein entscheidender Schritt besteht darin, vor dem Aufbringen des Photoresists die HSQ-Oberfläche einer Sauerstoffplasmabehandlung zu unterziehen - dies führt zur Bildung einer stabilen $\mathrm{SiO}_{2}$-Schicht auf dem HSQ und macht es so resistent gegenüber dem Photoresist-Lösungsmittel. Da die Nanodrähte auf dem Substrat statistisch verteilt sind, werden sie auch statistisch durch die Leiterbahnen adressiert. Nun lässt sich zwischen der Nanodrahtoberfläche und dem Substrat eine Spannung anlegen.

In Abbildung 6.10 a) - c) sind lichtmikroskopische Aufnahmen der prozessierten Proben zu sehen. Abbildung 6.10 a) zeigt eine Übersicht über ein prozessiertes Feld. Im zentralen Bereich des Bildes, in dem die Leiterbahnen aufeinander treffen, wirkt das Substrat leicht grünlich. Hier befindet sich die Öffnung in der isolierenden 
$\mathrm{SiO}_{2}$-Schicht. Im rechten Teil der Abbildung ist ein dunkler Draht zu sehen. Dabei handelt es sich um eine elektrische Zuleitung, die zu einem makroskopischen Kontakt führt. In Abbildung 6.10 b) ist eine Vergrößerung der elektrisch verbundenen Leiterbahn in dem zum p-Silizium geöffneten Bereich zu sehen. Neben der Leiterbahn ist auch ein Zinkoxid-Nanodraht in der Aufnahme zu erkennen. Im oberen Bereich des Bildes liegt ein größerer Kristallit über dem Draht. Unter einer Vielzahl der Leiterbahnen konnte nach der Prozessierung mindestens ein Nanodraht beobachtet werden. Wird nun eine Spannung zwischen Substrat und den Leiterbahnen auf den Nanodrähten angelegt, kann in der Tat eine Lichtemission an den Enden der Drähte beobachtet werden. Dies ist im Lichtmikroskop bei abgedimmter Beleuchtung, wie in Abbildung 6.10 c) dargestellt, gut zu beobachten.

\subsubsection{Leitfähigkeit und Elektrolumineszenz}

Eine spektrale Analyse des emittierten Lichts ist in Abbildung $6.11 \mathrm{c}$ ) dargestellt. Das Spektrum wurde bei Raumtemperatur unter Anlegen einer moderaten Spannung $\left(\approx 2 E_{g} / e\right)$ aufgenommen und wird von einer relativ schmalen Bande im UV-Bereich dominiert. Im Gegensatz zu anderen Untersuchungen [Bao06, Tsu05, Par04b, Sun06, Yan06] kann hier eine schwache Defektlumineszenz beobachtet werden. Im Einsatz von Abbildung 6.11 c) ist die Intensität der Lumineszenz als Funktion des Stroms für verschiedene prozessierte Nanodrähte aufgetragen. Die Mehrzahl der untersuchten Nanodrähte zeigt einen linearen Anstieg der Lumineszenz bei zunehmendem Stromfluss.

Ein entscheidender Schritt, um eine Elektrolumineszenz zu erhalten ist das Auftragen der dünnen Isolatorschicht auf das freigelegte p-Silizium, bevor die Nanodrähte auf die strukturierte Probe aufgebracht werden. Werden die Nanodrähte direkt auf das p-Silizium übertragen wird keine Elektrolumineszenz beobachtet. Abbildung 6.11 a) und b) zeigen Strom-Spannungskennlinien von mehreren kontaktierten Nanodrähten. In beiden Auftragungen ist ein gleichrichtendes Verhalten zu beobachten. Abbildung 6.11 a) zeigt sechs Kennlinien ohne die isolierende Schicht zwischen Nanodraht und p-Silizium. Alle Kennlinien zeigen eine gute Übereinstimmung, was auf die Reproduzierbarkeit des Prozesses hinweist. Der Strom in Durchlassrichtung beträgt etwa $30 \mu \mathrm{A}$ bei $2 \mathrm{~V}$. Auch bei deutlich höheren Strömen $(\approx 1 \mathrm{~mA})$ konnte 

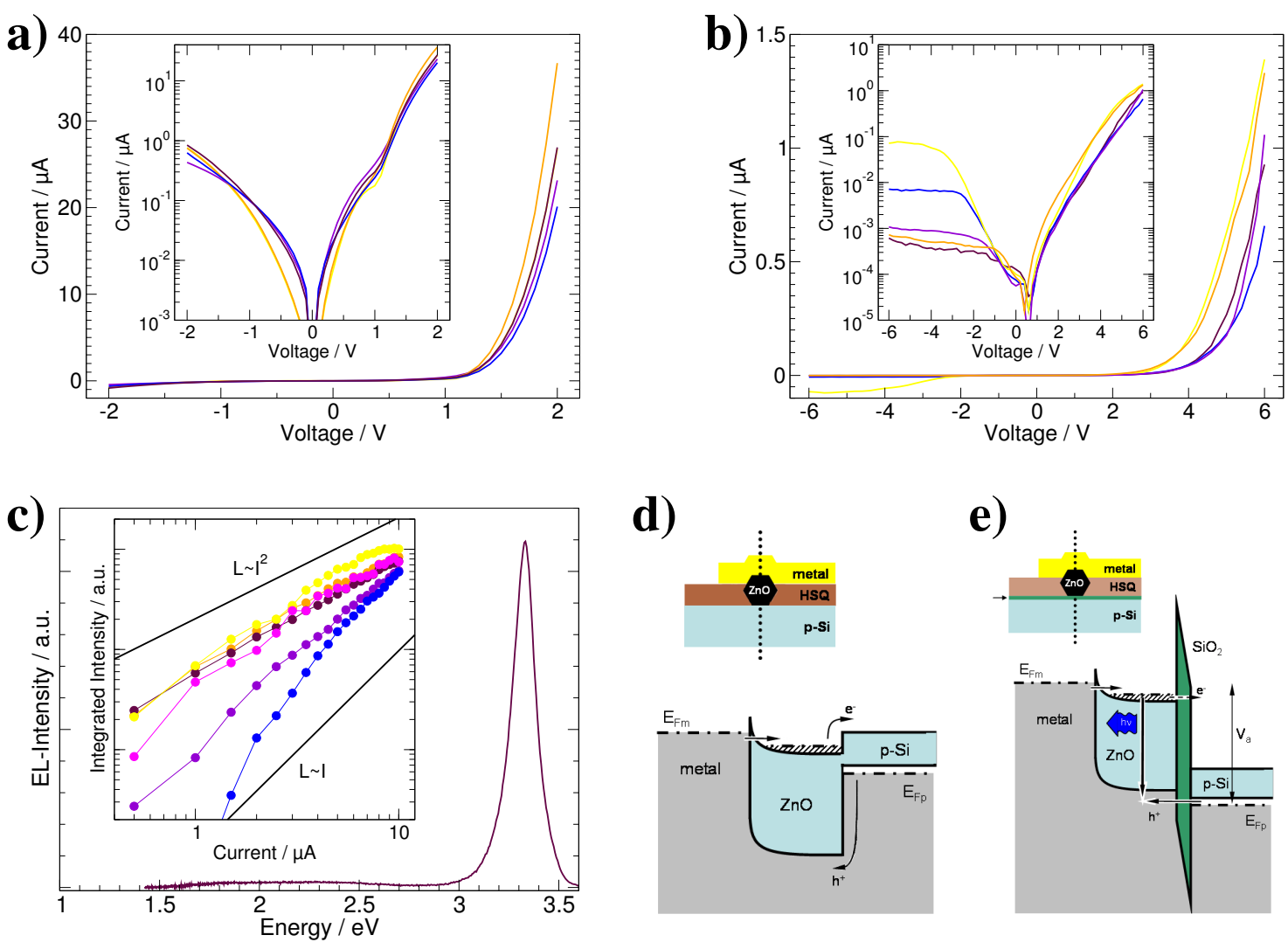

d)
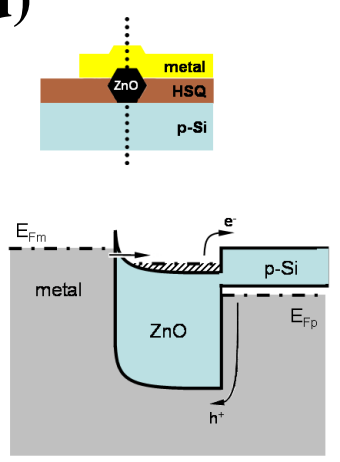

e)

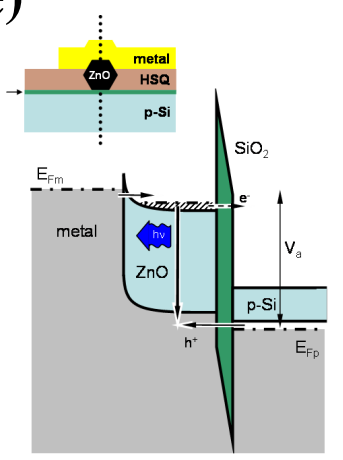

Abbildung 6.11: Charakteristik der präparierten n-Zinkoxid-Nanodrähte/p-SiliziumsubstratHeteroübergänge bei Raumtemperatur. a) Strom-Spannungskennlinien für sechs unterschiedliche Nanodrähte ohne Tunnelbarriere zwischen Nanodraht und Substrat. b) StromSpannungskennlinien für sechs unterschiedliche Nanodrähte mit einer $\approx 7 \mathrm{~nm}$ dicken Tunnelbarriere zwischen Nanodraht und Substrat. Im Einsatz sind die Kennlinien jeweils auf einer halblogarithmischen Skala aufgetragen. c) Elektrolumineszenzspektrum eines Zinkoxid-NanodrahtHeteroübergangs bei moderater Spannung. Im Einsatz ist die Intensität der Lumineszenz als Funktion des Stroms für sechs unterschiedliche Nanodrähte aufgetragen. d) und e) Bänderschemata für den Heteroübergang mit und ohne Tunnelbarriere.

keine Elektrolumineszenz beobachtet werden. Die Kennlinien des Heteroübergangs mit isolierender Zwischenschicht zeigt hingegen einen deutlich geringeren Strom von etwa $1 \mu \mathrm{A}$ bei $6 \mathrm{~V}$. An jedem untersuchten Nanodraht konnte eine Lichtemission beobachtet werden.

Diese Beobachtungen lassen sich mit den Bändermodellen für die beiden Versuchsgeometrien verstehen, die in Abbildung $6.11 \mathrm{~d}$ ) und e) dargestellt sind. In Abbildung $6.11 \mathrm{~d}$ ) ist die Anordnung ohne isolierende Zwischenschicht dargestellt [Sun06]. 
In diesem Fall beträgt die Barrierenhöhe für die Injektion von Löchern in das Zinkoxid $\approx 2,5 \mathrm{eV}$. Wird eine Spannung angelegt ändert sich die Barrierenhöhe allerdings nicht, wodurch eine Injektion von Löchern nicht möglich ist. Entsprechend wird auch kein Licht emittiert. Wird nun ein Dielektrikum zwischen die beiden Halbleiter gebracht kann über dessen Dicke ein großer Potentialunterschied aufrechterhalten werden. Entsprechend können Elektronen aus dem Valenzband des Zinkoxids durch das Dielektrikum in das Silizium tunneln. Dies entspricht einer Injektion von Löchern in das Zinkoxid in entgegengesetzte Richtung. Durch eine Rekombination der injizierten Löcher mit Leitungsbandelektronen im Zinkoxid kommt es zu einer Emission von UV-Licht. Ein ähnlicher Prozess wurde bei einem Heteroübergang zwischen nGalliumnitrid-Nanodrähten und p-Silizium vorgeschlagen, wobei dort die natürliche Oxidschicht auf dem Substrat sowie um die Galliumnitrid-Nanodrähte als Tunnelbarriere diente [Zim07b, Zim07a].

\subsection{Zusammenfassung}

In diesem Kapitel wurden Experimente vorgestellt, die im Zusammenhang mit den elektrischen Eigenschaften und der Funktionalisierung von Halbleiternanodrähten als elektronische und optoelektronische Bauteile stehen.

Im ersten Abschnitt wurde am Beispiel von Galliumarsenid gezeigt, dass sich Halbleiternanodrähten mittels Ionenimplantation elektrisch dotieren lassen. Dies ist von entscheidender Bedeutung, da eine Dotierung während der Synthese sich insbesondere aufgrund des Wachstumsmechanismus als außergewöhnlich schwierig erweist. Eine Abschätzung der Ladungsträgerkonzentration aus der Leitfähigkeit der Nanodrähte zeigt, dass ein Großteil der implantierten Zinkionen elektrisch aktiv ist.

Im zweiten Abschnitt wurde gezeigt, dass sich Zinkoxid-Nanodrähte vergleichsweise einfach zu Feldeffekttransistoren prozessieren lassen, indem ein leitfähiges Substrat mit isolierender Zwischenschicht als Basis und Kontakte an den Enden eines Nanodrahtes als Emitter und Kollektor dienen. Die Nanodrähte haben nach der Synthese eine relativ große Hintergrundkonzentration von Donatoren. Dies legt die Klassifizierung des Feldeffekttransistors als n-Kanal-Verarmungstyp fest. Bestätigt wird diese Klassifizierung durch eine negative Einsatzspannung und eine zunehmende Leitfähigkeit mit steigender Basisspannung positiver Polarität. 
Ein Vergleich zwischen Feldeffekttransistoren bei denen Nanodrähte mit einem Durchmesser von $100 \mathrm{~nm}$ und Nanodrähte mit $18 \mathrm{~nm}$ Durchmesser als aktiver Kanal verwendet wurde zeigt, dass die Feldeffekttransistoren mit geringerem Durchmesser des aktiven Kanals eine deutlich größere Ladungsträgerkonzentration, gleichzeitig aber auch eine stark reduzierte Ladungsträgermobilität aufweisen. Die jeweiligen Größen nehmen annähernd Werte an, die typisch für Metalle sind. Diese Beobachtung wird auf einen zunehmenden Einfluss von Oberflächenzuständen zurückgeführt, für die insbesondere eine geringe Ladungsträgermobilität typisch ist. Dies äußert sich in den Ausgangskennlinien in einem sehr geringen Einfluss der Basisspannung auf die Leitfähigkeit des Nanodrahtes.

Da die Oberfläche von Zinkoxid die Leitfähigkeit stark beeinflusst findet Zinkoxid Anwendung in der Gassensorik. Als Ursache für die Änderung der Leitfähigkeit wird in diesem Bereich insbesondere auf eine Veränderung des Oberflächenpotenzials durch die Wechselwirkung von Sauerstoffleerstellen mit Adsorbaten verwiesen. Da sich der Effekt von Oberflächendefekten und -potenzialen negativ auf die Leistungsmerkmale eines Feldeffekttransistors auswirkt wurde eine Passivierung und Abdeckung der Nanodrahtoberfläche vorgenommen. In der Tat konnten durch dieses Vorgehen die Leistungsmerkmale des Transistors deutlich verbessert werden. Im Detail wurde neben einer um zwei Größenordnungen höheren Ladungsträgermobilität vor allem auch eine um zwei Größenordnungen höhere Transkonduktanz und ein um die Hälfte reduzierter Subthresholdswing ermittelt.

Das einzige Leistungsmerkmal, das für eine technische Relevanz eines solchen Feldeffekttransistors absolut ungeeignet ist, ist die große negative Schwellenspannung. Diese kommt geometriebedingt durch den geringen elektrostatischen Einfluss der Basis auf die Ladungsträger im Nanodraht zustande. Entsprechend wurde in einem weiteren Experiment ein Nanodraht-Feldeffekttransistor in Topgate-Geometrie hergestellt. Hier konnten deutlich geringere Einsatzspannungen im Bereich von -0,2 V realisiert werden. Auch der Wert des Subthresholdswing konnte um einen Faktor fünf weiter verbessert werden und befindet sich in einem Bereich, in den gerade auch gute CMOS-basierte Feldeffekttransistoren vorstoßen. Allerdings sind bei dem in Topgate-Geometrie gefertigten Bauteil die übrigen Werte, insbesondere die Ladungsträgermobilität, etwas schlechter als bei dem zuvor gefertigten Bauteil. Dies wurde insbesondere durch einen abweichenden Herstellungsprozess der Nanodrähte 
begründet.

Im letzten Abschnitt dieses Kapitels wurde ein einfacher Prozess zur großflächigen Herstellung von individuell adressierbaren Leuchtdioden vorgestellt, die auf einem Heteroübergang zwischen n-Zinkoxid-Nanodrähten und p-Silizium beruhen. Es wurde gezeigt, dass das Elektrolumineszenzspektrum von einer Bande im bandkantennahen Bereich dominiert wird, dessen Intensität wie erwartet linear mit dem Strom zunimmt. Die Strom-Spannungskennlinien der Heterostruktur zeigen ein gleichrichtendes Verhalten. Es konnte weiterhin gezeigt werden, dass eine dünne isolierende Schicht zwischen dem Halbleiternanodraht und dem p-Silizium eine entscheidende Rolle für die Lichtemission spielt. Es wird angenommen, dass die Elektrolumineszenz auf einer Tunnelinjektion von Löchern in das Zinkoxid beruht. 
146 Funktionalisierung VON NANOdRÄHTEN ALS ELEKTRONiSChe BAUELEMENTE 


\section{Kapitel 7}

\section{Zusammenfassung und Ausblick}

In dieser Arbeit wurde die Synthese, Charakterisierung und Funktionalisierung von Halbleiternanodrähten dargestellt und diskutiert. Dabei wurden insbesondere die Materialsysteme Zinkoxid, Zinksulfid und Galliumarsenid untersucht. Es wurde gezeigt, dass sich Halbleiternanodrähte in einem relativ einfachem Prozess herstellen lassen. Der Wachstumsprozess beruht auf dem von Wagner und Ellis vorgeschlagenen VLS-Mechanismus. Für die unterschiedlichen Materialsysteme und Syntheseprozesse zur Herstellung der Nanodrähte wurde die Gültigkeit des VLSMechanismus diskutiert und zum Teil Abwandlungen vorgeschlagen. Die synthetisierten Nanodrähte haben die Form von Nanodrähten und Nanobändern mit einem Durchmesser zwischen $4 \mathrm{~nm}$ und $200 \mathrm{~nm}$.

An Zinkoxid-Nanodrähten wurden detaillierte und systematische optische Untersuchungen vorgestellt. Es konnte gezeigt werden, dass Lumineszenzspektren von Zinkoxid-Nanodrahtensemblen mit einem mittleren Durchmesser von $100 \mathrm{~nm}$ im Wesentlichen durch scharfe Banden im bandkantennahen Bereich geprägt sind, die auf donatorgebundene Exzitonen zurückzuführen sind. Eine Lumineszenz tiefer Störstellen blieb größtenteils aus, was auf eine hohe Güte der hergestellten Strukturen hindeutet. Durch diese Eigenschaften unterscheiden sich die Lumineszenzspektren von Nanodrähten nicht zu sehr von Volumenkristallen. In Lumineszenzuntersuchungen an einzelnen Nanodrähten konnte gezeigt werden, dass die optischen Untersuchungen am Ensemble die wesentlichen Merkmale der einzelnen Nanodrähte wiedergeben, was auf homogene Proben hinweist.

Bei der optischen Charakterisierung der Nanodrähte konnten hingegen auch Di- 
mensionseffekte beobachtet werden - das heißt Eigenschaften, durch die sich die Nanodrähte von Volumenkristallen unterscheiden. In systematischen Photolumineszenzuntersuchungen an Zinkoxid-Nanodrähten mit unterschiedlichem Durchmesser wurde die zunehmende Dominanz einer asymmetrischen Lumineszenzbande bei 3,366 eV bei kleiner werdendem Drahtdurchmesser beobachtet. Diese Lumineszenz wurde Exzitonen zugeordnet, die in einer Mantelschicht des Drahtes an unterschiedlichen Defekten gebunden sind, wobei die Defekte im Zusammenhang mit der Oberfläche der Nanodrähte stehen. Dieser Effekt ist folglich als Einfluss des vergrößerten Oberflächen-zu-Volumenverhältnis auf die exzitonische Lumineszenz zu verstehen. Ultradünne Nanodrähte mit einem Durchmesser von $\approx 4 \mathrm{~nm}$ zeigten Hinweise auf ein Quantum confinement. Weiterhin konnte gezeigt werden, dass der nichtlineare optische Prozess der Exziton-Exzitonstreuung bei dünnen Nanodrähten aufgrund der reduzierten Geometrie effektiv unterdrückt wird. In Untersuchungen an ZinkoxidNanodrähten, die mittels Ionenimplantation mit Stickstoff dotiert wurden, konnte nach einer Temperaturbehandlung ein Donator-Akzeptor-Paarübergang beobachtet werden. In vergleichenden, detaillierten Untersuchungen an Volumenkristallen konnte dieser eindeutig als solcher identifiziert werden.

Die Veränderung der Morphologie und der Kristallinität durch Ionenimplantation sowie das Ausheilen von Kristalldefekten in einer Temperaturbehandlung wurde detailliert an den Materialsystemen Zinkoxid und Galliumarsenid vorgestellt.

Nach der Bestrahlung von Zinkoxid-Nanodrähten mit schweren Ionen niedriger Energie und hoher Dosis konnte eine charakteristische strukturelle Veränderung der Nanodrähte beobachtet werden. Diese konnte in erster Näherung mit einem Sputtermodell beschrieben werden. Obgleich eine hohe Ionendosis verwendet wurde, konnte keine Amorphisierung der Nanodrähte beobachtet werden. Dies wurde auf die hohe Ionizität der Bindung zurückgeführt. Außerdem konnte gezeigt werden, dass die eingebrachten Ionen auch nach der Temperaturbehandlung in der erwünschten Konzentration in den Nanodrähten vorhanden sind.

Im Gegensatz zu den implantierten Zinkoxid-Nanodrähten wurde nach der Implantation von Galliumarsenid-Nanodrähten mit vergleichsweise leichten Ionen höherer Energie und geringerer Dosis eine nahezu vollständige Amorphisierung beobachtet. Außerdem wurde beobachtet, dass sich die Nanodrähte in Richtung des Ionenstrahls ausrichten. Nach einer geeigneten Temperaturbehandlung konnte eine vollständige 
Rekristallisation der Nanodrähte erreicht werden - lediglich einige Versetzungen blieben zurück.

Weiterhin wurde gezeigt, dass sich durch Ionenimplantation aktive Leuchtzentren in Nanostrukturen erzeugen lassen. Im Detail wurden Untersuchungen an mit Seltenen Erden implantierten Zinkoxid-Nanodrähten und an mit Mangan implantierten Zinksulfid-Nanodrähten und -bändern vorgestellt. In beiden Systemen konnte eine optische Aktivierung von internen Übergängen im 3d- bzw. 4f-System der Dotanden beobachtet werden. Die Lumineszenz der mit Seltenen Erden implantierten Zinkoxid-Nanodrähte ist vergleichsweise schwach ausgeprägt und die Charakteristik verweist auf gestörte lokale Umgebungen der Dotanden im Kristallgefüge. Im Gegensatz dazu zeigen die mit Mangan implantierten Zinksulfid-Strukturen eine intensive Mangan-intra-3d-Lumineszenz. In systematischen Untersuchungen der Lebenszeit dieser Lumineszenz, bei der sowohl die Mangankonzentration als auch die Morphologie der Nanokristalle variiert wurde, zeigte sich ein starker Einfluss der Morphologie der Zinksulfid-Nanostrukturen. Es wurde gezeigt, dass sich das zeitliche Abklingen dieser Lumineszenz für alle untersuchten Nanostrukturen mit einer Abwandlung des Förstermodells hinsichtlich der Dimension der Wirtskristalle konsistent beschreiben lässt.

Desweiteren wurde gezeigt, dass sich auch die elektrischen Eigenschaften von Halbleiternanodrähten durch Ionenimplantation modifizieren lassen. Im Experiment wurden Zinkatome mittels Ionenimplantation in Galliumarsenid-Nanodrähte eingebracht. Es wurde gezeigt, dass sich die Leitfähigkeit der Nanodrähte nach einer geeigneten Temperaturbehandlung um Größenordnungen ändert, was auf eine elektrische Aktivierung der Zinkatome als Akzeptoren zurückgeführt wurde. Eine Abschätzung der Ladungsträgerkonzentration aus der Leitfähigkeit zeigte, dass ein Großteil der Zinkatome elektrisch aktiv ist.

Weiterhin wurde gezeigt, dass sich Zinkoxid-Nanodrähte vergleichsweise einfach zu Feldeffekttransistoren prozessieren lassen. Für die Leistungsmerkmale des Transistors ist essentiell, dass die Oberfläche der Nanodrähte passiviert ist. Dies wurde insbesondere an Untersuchungen deutlich, bei denen dünne Nanodrähte mit entsprechend großem Oberflächen-zu-Volumenverhältnis zu Feldeffekttransistoren prozessiert wurden. Die Oberflächenpassivierung wurde durch die Einbettung des Nanodrahtes in ein Dielektrikum realisiert. Außerdem ist für die Leistungsmerkma- 
le des Transistors ein möglichst großer elektrostatischer Einfluss der Basiselektrode auf den aktiven Kanal wichtig. Dies konnte durch die Verwendung einer TopgateGeometrie realisiert werden. Die hergestellten Transistoren zeigten hervorragende Eigenschaften.

Schließlich wurde ein einfaches Verfahren vorgestellt, mit dessen Hilfe sich großflächig Zinkoxid-Nanodraht-basierte Leuchtdioden herstellen lassen. Bei dem Verfahren kommt lediglich optische Lithographie zum Einsatz. Das Funktionsprinzip der Leuchtdioden basiert auf einem Heteroübergang zwischen p-Silizium und nZinkoxid-Nanodrähten, wobei sich zwischen den Materialien ein dünnes Dielektrikum befindet. Auf diese Weise kommt es bei dem Anlegen einer Spannung zu einer Löcherinjektion aus dem p-Silizium in die Nanodrähte und somit zu einer Lichtemission im ultravioletten Spektralbereich.

Obgleich hier ein skalierbarer und einfacher Prozess zur Herstellung einer Vielzahl von Leuchtdioden vorgestellt wurde, so bleibt die großfächige und gezielte Adressierung von Nanodrähten mit hoher Integrationsdichte die zentrale Problematik. Entsprechend wäre es naiv anzunehmen, dass Nanodraht-basierte Elektronik zukünftigt die CMOS-Technologie verdrängen könnte. Vielmehr wird angenommen, dass die Nanodraht-basierte Technologie ihre Anwendung in speziellen Gebieten findet, wo ihre Eigenschaften überlegen sind. Das Beispiel der Nanodraht-basierten Leuchtdioden kann ein solches Gebiet sein - eine Lichtquelle auf dieser Größenskala lässt sich mit reinen top-down-Methoden vermutlich nicht realisieren. An dieser Stelle kann sicher angeknüpft werden, um Nano-Lichtquellen in unterschiedlichen Wellenlängenbereichen herzustellen, beispielsweise unter Verwendung von Leuchtzentren wie den Seltenen Erden oder Mangan. Diese Systeme weisen vergleichsweise lange Lebenszeiten der internen Übergänge auf. Dies ermöglicht eine einfachere Besetzungsinversion und ermöglicht so eine stimulierte Emission, wie sie sich in leistungsabhängigen Lumineszenzmessungen an mit Seltenen Erden implantierten Zinkoxid-Nanodrähten abzeichnete. Zusammen mit dem vorgestellten Verfahren zur Herstellung der Leuchtdioden führt dies näher zu elektrisch gepumpten Nanolasern einstellbarer Wellenlänge. Dabei können die Nanodrähte selbst als Kavität wirken. Der Prozess zur Herstellung der Nanodraht-Leuchtdioden zeigt ebenfalls, dass die Nanodraht-Technologie mit Standard-CMOS Methoden und der Siliziumtechnologie kombinierbar ist und somit von Fortschritten in diesem Bereich mit profitiert. 


\section{Literaturverzeichnis}

[Ada04] M. Adachi, Y. Murata, J. Takao, J. Jiu, M. Sakamoto, und F. Wang, Highly Efficient Dye-Sensitized Solar Cells with a Titania Thin-Film Electrode Composed of a Network Structure of Single-Crystal-Like TiO 2 Nanowires Made by the Oriented Attachment Mechanism, J. Am. Chem. Soc. 126, 14943 (2004).

[Ali96] A. P. Alivisatos, Semiconductor Clusters, Nanocrystals, and Quantum Dots, Science 271, 933 (1996).

[Alv03] E. Alves, E. Rita, U. Wahl, J. G. Correia, T. Monteiro, J. Soares, und C. Boemare, Lattice Site Location and Optical Activity of Er Implanted ZnO, Nucl. Instrum. Meth. B 206, 1047 (2003).

[Ari05] A. S. Arico, P. Bruce, B. Scrosati, J. M. Tarascon, und W. van Schalkijk, Nanostructured Materials for Advanced Energy Conversion and Storage Devices, Nature Materials 4, 366 (2005).

[Aur01] F. D. Auret, S. A. Goodman, M. Hayes, M. J. Legodi, H. A. van Laarhoven, und D. C. Look, The Influence of High Energy Proton Bombardment on the Electrical and Defect Properties of Single-Crystal ZnO, J. Phys.: Condens. Matter 13, 8989 (2001).

[Bac93] S. Bachir, J. C. Ronfasrd-Haret, K. Azuma, D. Kouyate, und J. Kossanyi, Direct Impact Excitation of Thulium(III) Luminescence in Polycrystalline $\mathrm{ZnO}: \mathrm{Tm}^{3+}$ Electrodes in Contact with Aqueous Electrolyte, and Attribution of the Luminescence Spectrum, Chem. Phys. Lett. 213, 54 (1993).

[Bac00] A. Bachtold, P. Hadley, T. Nakanishi, und C. Dekker, Logic Circuits with Carbon Nanotube Transistors, Science 294, 1317 (2000).

[Bao06] J. Bao, M. A. Zimmler, F. Capasso, X. Wang, und Z. F. Ren, Broadband ZnO Single-Nanowire Light-Emitting Diode, Nano Lett. 6, 1719 (2006). 
[Bau99] R. H. Baughman, Carbon Nanotube Actuators, Science 284, 1340 (1999).

[Bäu00] P. Bäume, M. Behringer, J. Gutowski, und D. Hommel, Calculation of the Coulomb Broadening of Donor-Acceptor Pair Emission in Compensated Semiconductors, Phys. Rev. B 62, 8023 (2000).

[Bha94a] R. N. Bhargava, D. Gallagher, X. Hong, und A. Nurmikko, Optical Properties of Manganese-Doped Nanocrystals of ZnS, Phys. Rev. Lett. 72, 416 (1994).

[Bha94b] R. N. Bhargava, D. Gallagher, und T. Welker, Doped Nanocrystals of Semiconductors - a New Class of Luminescent Materials, J. Lumin. 60, 275 (1994).

[Bha96] R. N. Bhargava, Doped Nanocrystalline Materials - Physics and Applications, J. Lumin. 70, 85 (1996).

[Bjö02] M. T. Björk, B. J. Ohlsson, T. Sass, A. I. Persson, C. Thelander, M. H. Magnusson, K. Deppert, L. R. Wallenberg, und L. Samuelson, OneDimensional Steeplechase for Electrons Realized, Nano Lett. 2, 87 (2002).

[Bol98] A. A. Bol und A. Meijerink, Long-Lived $\mathrm{Mn}^{2+}$ Emission in Nanocrystalline ZnS:Mn ${ }^{2+}$, Phys. Rev. B 58, R15 997 (1998).

[Bol00] A. A. Bol und A. Meijerink, Doped Semiconductor Nanoparticles - a New Class of Luminescent Materials?, J. Lumin. 87, 315 (2000).

[Bor99] P. H. Borse, D. Srinivas, R. F. Shinde, S. K. Date, W. Vogel, und S. K. Kulkarni, Effect of $\mathrm{Mn}^{2+}$ Concentration in ZnS Nanoparticles on Photoluminescence and Electron-Spin-Resonance Spectra, Phys. Rev. B 60, 8659 (1999).

[Bor06a] C. Borchers, S. Müller, D. Stichtenoth, D. Schwen, und C. Ronning, Catalyst-Nanostructure Interaction in the Growth of 1-D ZnO Nanostructures, J. Phys. Chem. B 110, 1656 (2006).

[Bor06b] C. Borchers, D. Stichtenoth, S. Müller, D. Schwen, und C. Ronning, Catalyst-Nanostructure Interaction and Growth of ZnS Nanobelts, Nanotechnology 17, 1067 (2006).

[Bou07] G. Boulon und V. Lupei, Energy Transfer and Cooperative Processes in $\mathrm{Yb}^{3+}{ }_{-}$Doped Cubic Sesquioxide Laser Ceramics and Crystals, J. Lumin. 125, 45 (2007). 
[Boy88] R. Boyn, 4f-4f Luminescence of Rare-Earth Centers in II-VI Compounds, Phys. Stat. Sol. B 148, 11 (1988).

[But98] T. V. Butkhuzi, T. G. Chelidze, A. N. Georgobiani, D. L. Jashiashvili, T. G. Khulordava, und B. E. Tsekvava, Exciton Photoluminescence of Hexagonal ZnO, Phys. Rev. B 58, 10692 (1998).

[Car68] W. T. Carnall, P. R. Fields, und K. Rajnak, Electronic Energy Levels of the Trivalent Lanthanide Aquo Ions IV. Eu ${ }^{3+}$, J. Chem. Phys. 49, 4450 (1968).

[Cas06] C. Cascales, M. D. Serrano, F. Esteban-Betegon, C. Zaldo, R. Peters, K. Petermann, G. Huber, L. Ackermann, D. Rytz, C. Dupre, M. Rico, J. Liu, U. Griebner, und V. Petrov, Structural, Spectroscopic, and Tunable Laser Properties of $\mathrm{Yb}^{3+}$-Doped $\mathrm{NaGd}\left(\mathrm{WO}_{4}\right)_{2}$, Phys. Rev. B 74, 174114 (2006).

[Cha04] P. C. Chang, Z. Fan, D. Wang, W. Y. Tseng, W. A. Chiou, J. Hong, und J. G. Lu, ZnO Nanowires Synthesized by Vapor Trapping CVD Method, Chem. Mater. 16, 5133 (2004).

[Cha06] P. C. Chang, Z. Fan, C. J. Chien, D. Stichtenoth, C. Ronning, und J. G. Lu, High-Performance ZnO Nanowire Field Effect Transistors, Appl. Phys. Lett. 89, 133113 (2006).

[Cha07a] P. C. Chang, C. J. Chien, D. Stichtenoth, C. Ronning, und J. G. Lu, Finite Size Effect in ZnO Nanowires, Appl. Phys. Lett. 90, 113101 (2007).

[Cha07b] T. Chavanapranee und Y. Horikoshi, Characterization of Heavily SnDoped GaAs Grown by Migration-Enhanced Epitaxy, J. Cryst. Growth 301, 225 (2007).

[Che92] J. Chevallier, B. Machayekhi, C. M. Grattepain, R. Rahbi, und B. Theys, Diffusion and Electric States of Hydrogen in n-Type GaAs and n-Type Ga $a_{1-x} A l_{x} A s$, Phys. Rev. B 45, 8803 (1992).

[Che00] W. Chen, J. O. Malm, V. Zwiller, Y. Huang, S. Liu, R. Wallenberg, J. O. Bovin, und L. Samuelson, Energy Structure and Fluorescence of Eu ${ }^{2+}$ in ZnS:Eu Nanoparticles, Phys. Rev. B 61, 11021 (2000).

[Che01] W. Chen, R. Sammynaiken, Y. Huang, J. O. Malm, R. Wallenberg, J. O. Bovin, V. Zwiller, und N. A. Kotov, Crystal Field, Phonon Coupling and Emission Shift of $\mathrm{Mn}^{2+}$ in ZnS:Mn Nanoparticles, J. Appl. Phys. 89, 1120 (2001). 
[Che02] Y. Chen, J. Li, Y. Han, X. Yang, und J. Dai, The Effect of Mg Vapor Source on the Formation of MgO Whiskers and Sheets, J. Cryst. Growth 245, 163 (2002).

[Che05] W. Chen, V. F. Aguekian, N. Vassiliev, A. Y. Serov, und N. G. Filosofov, New Observations on the Luminescence Decay Lifetime of $\mathrm{Mn}^{2+}$ in ZnS:Mn ${ }^{2+}$ Nanoparticles, J. Chem. Phys. 123, 124707 (2005).

[Che07] L. Chen, F. J. Brieler, M. Fröba, P. J. Klar, und W. Heimbrodt, Quantitative Description of the Temporal Behavior of the Internal Mn $3 d^{5}$ Luminescence in Ensembles of $Z n_{0.99} M n_{0.01} S$ Quantum Dots, Phys. Rev. $B$ 75, 241303(R) (2007).

[Col99] C. P. Collier, E. W. Wong, M. Belohradski, F. M. Raymo, J. F. Stoddart, P. J. Kuekes, R. S. Williams, und J. R. Heath, Electronically Configurable Molecular-Based Logic Gates, Science 285, 391 (1999).

[Col00] P. G. Collins und P. Avouris, Nanotubes for Electronics, Sci. Am. 283, $62(2000)$.

[Col01] P. G. Collins, M. S. Arnold, und P. Avouris, Engineering Carbon Nanotubes and Nanotube Circuits Using Electrical Breakdown, Science 292, 706 (2001).

[Cos04] C. Coskun, D. C. Look, G. C. Farlow, und J. R. Sizelove, Radiation Hardness of ZnO at Low Temperatures, Semicond. Sci. Technol. 19, 752 (2004).

[Cry] Personal communication, CrysTec GmbH, Berlin.

[Cui01a] Y. Cui, X. Duan, J. Hu, und C. M. Lieber, Doping and Electrical Transport in Silicon Nanowires, J. Phys. Chem. B 104, 5213 (2001).

[Cui01b] Y. Cui, Q. Wei, H. Park, und C. M. Lieber, Nanowire Nanosensors for Highly Sensitive and Selective Detection of Biological and Chemical Species, Science 293, 1289 (2001).

[Cui03] Y. Cui, Z. Zhong, D. Wang, W. U. Wang, und C. M. Lieber, High Performance Silicon Nanowire Field Effect Transistor, Nano Lett. 3, 149 (2003).

[Dai96] H. Dai, J. H. Hafner, A. G. Rinzler, D. T. Colbert, und R. E. Smalley, Nanotubes as Nanoprobes in Scanning Probe Microscopy, Nature 384, 147 (1996). 
[DB04] (15. Wahlperiode) Deutscher Bundestag, Bericht des Ausschusses für Bildung, Forschung und Technikfolgenabschätzung - TA Projekt Nanotechnologie, Bundestags-Durcksache 15/2713 (2004).

[Der01] V. Derycke, R. Martel, J. Appenzeller, und P. Avouris, Carbon Nanotube Inter- and Intramolecular Logic Gates, Nano Lett. 1, 453 (2001).

[Dev00] M. H. Devoret und R. J. Schoelkopf, Amplifying Quantum Signals with the Single-Electron Transistor, $N$ 406, 1039 (2000).

[Dew07] W. Dewald, Synthese und Charakterisierung von Silizium-Nanodrähten, Diplomarbeit (2007).

[Dex53] D. L. Dexter, A Theory of Sensitized Luminescence in Solids, J. Cryst. Growth 21, 836 (1953).

[Die68] G. H. Diecke, Spectra and Energy Levels of Rare Earth Ions in Crystals, Wiley-Interscience, New York (1968).

[Dou06] T. Douglas und M. Young, Viruses: Making Friends with Old Foes, Science 312, 873 (2006).

[Dua00] X. Duan und C. M. Lieber, General Synthesis of Compound Semiconductor Nanowires, Adv. Mater. 12, 298 (2000).

[Dua01a] X. Duan, Y. Huang, Y. Cui, J. Wang, und C. M. Lieber, Indium Phosphide Nanowires as Building Blocks for Nanoscale Electronic and Optoelectronic Devices, Nature 409, 66 (2001).

[Dua01b] X. Duan, Y. Huang, Y. Cui, J. Wang, und C. M. Lieber, Indium Phosphide Nanowires as Building Blocks for Nanoscale Electronic and Optoelectronic Devices, Nature 409, 66 (2001).

[Dua03] X. Duan, Y. Huang, R. Agarwal, und C. M. Lieber, Single-Nanowire Electrically Driven Lasers, Nature 421, 241 (2003).

[DV92] P. De Visschere und K. Neyts, Concentration Quenching and Luminescent Decay in AC Thin-Film ZnS:Mn Electroluminescent Devices, J. Lumin. 52, 313 (1992).

[Era04] G. Eranna, B. C. Joshi, D. P. Runthala, und R. P. Gupta, Oxide Materials for Development of Integrated Gas Sensors - a Comprehensive Review, Crit. Rev. Solid State Mater. Sci. 29, 111 (2004). 
[Eve60] T. E. Everhart und R. F. M. Thornley, Wide-Band Detector for MicroMicroampere Low-Energy Electron Currents, J. Sci. Instr. 37, 246 (1960).

[Fan04] Z. Fan, D. Wang, P. C. Chang, W. Y. Tseng, und J. G. Lu, ZnO Nanowire Field-Effect Transistor and Oxygen Sensing Property, Appl. Phys. Lett. 85, 5923 (2004).

[Fan06] H. J. Fan, P. Werner, und M. Zacharias, Semiconductor Nanowires: from Self-Organization to Patterned Growth, Small 2, 700 (2006).

[Far06] O. C. Farokhzad, J. Cheng, B. A. Teply, I. Shrifi, S. Jon, P. W. Kantoff, J. P. Richie, und R. Langer, Targeted Nanoparticle-Aptamer Bioconjugates for Cancer Chemotherapy in Vivo, Proc. Natl. Acad. Sci. USA 103, 6315 (2006).

[Fey59] R. P. Feynman, There Is Plenty of Room at the Bottom (1959), lecture given at the annual meeting of the American Physical Society at Caltech.

[Fis69] V. I. Fistul, Heavily Doped Semiconductors, Plenum, New York (1969).

[Fon06] P. Fons, H. Tampo, A. V. Kolobov, M. Ohkubo, S. Niki, und J. Tominaga, Direct Observation of Nitrogen Location in Molecular Beam Epitaxy Grown Nitrogen-Doped ZnO, Phys. Rev. Lett. 96, 045504 (2006).

[För49] T. Förster, Experimetelle und Theoretische Untersuchungen des Zwischenmolekularen Übergangs von Elektronenanregungsenergie, Z. Naturforschung 4, 321 (1949).

[Fre31] S. Freed, Electronic Transitions Between an Inner Shell and the Virtual Outer Shells of the Ions of the Rare Earths in Crystals, Phys. Rev 38, 2122 (1931).

[Fuh05] B. Fuhrmann, H. S. Leipner, und H. R. Höche, Ordered Arrays of Silicon Nanowires Produced by Nanosphere Lithography and Molecular Beam Epitaxy, Nano Lett. 5, 2524 (2005).

[Geb08] S. Geburt, D. Stichtenoth, S. Müller, W. Dewald, C. Ronning, J. Wang, Y. Jiao, Y. Y. Rao, S. K. Hark, und Q. Li, Rare Earth Doped Zinc Oxide Nanowires, J. Nanosci. Nanotechnol. 8, 244 (2008). 
[Geo77] A. N. Georgobiani, B. P. Dementev, M. B. Kotlyarevskii, und P. E. Ramazanov, Investigation of Defect Formation in ZnS Single Crystals Following Ionic Implantation of Compound Components, Russ. Phys. J. 20, 1305 (1977).

[Gra76] M. Grade, W. Hirschwald, und F. Stolze, Z. Phys. Chem. Neue Folge 100, 165 (1976).

[Gra05] J. Grabowska, A. Meaney, K. K. Nanda, J. P. Mosnier, M. O. Henry, J. R. Duclere, und E. McGlynn, Surface Excitonic Emission and Quenching Effects in ZnO Nanowire/nanowall Systems: Limiting Effects on Device Potential, Phys. Rev. B 71, 115439 (2005).

[Gud02] M. S. Gudiksen, L. J. Lauhon, J. Wang, D. Smith, und C. M. Lieber, Growth of Nanowire Superlattice Structures for Nanoscale Photonics and Electronics, Nature 415, 617 (2002).

[Gum81] H. E. Gumlich, Electro- and Photoluminescence Properties of $\mathrm{Mn}^{2+}$ in ZnS and ZnCdS, J. Lumin. 23, 73 (1981).

[Han52] H. Hanemann und A. Schrader, Ternäre Legierungen des Aluminiums, Atlas Metallographicus, III.2, Verlag Stahleisen MbH, Düsseldorf, Germany (1952).

[Han07] M. Hanke, C. Eisenschmidt, P. Werner, N. D. Zakharov, F. Syrowatka, F. Heyroth, P. Schäfer, und O. Konovalov, Elastic Strain Relaxation in Axial Si/Ge Whisker Heterostructures, Phys. Rev. B 75, 161303(R) (2007).

[Hau06a] R. Hauschild, ZnO-Nanosäulen als Laseremitter, Dissertation (2006).

[Hau06b] R. Hauschild und H. Kalt, Guided Modes in ZnO Nanorods, Appl. Phys. Lett. 89, 123107 (2006).

[Hau06c] R. Hauschild, H. Priller, M. Decker, J. Brückner, H. Kalt, und C. Klingshirn, Temperature Dependent Band Gap and Homogeneous Line Broadening of the Exciton Emission in ZnO, Phys. Stat. Sol. C 3, 976 (2006).

[Hec86] N. Hecking, K. F. Heidemann, und E. Te Kaat, Model of Temperature Dependent Defect Interaction and Amorphization in Crystalline Silicon During Ion Irradiation, Nucl. Instrum. Meth. B 15, 760 (1986). 
[Heo04] Y. W. Heo, L. C. Tien, Y. Kwon, D. P. Norton, S. J. Pearton, B. S. Kang, und F. Ren, Depletion-Mode ZnO Nanowire Field-Effect Transistor, Appl. Phys. Lett. 85, 2274 (2004).

[Hom90] D. Hommel, W. Busse, H. E. Gumlich, D. Suisky, J. Röseler, K. Swiatek, und M. Godlewski, Rare Earths in II-VI Compounds: Non-Linear Optical Excitation Processes at Low and High Doping Levels, J. Cryst. Growth 101, 393 (1990).

[Hom92] D. Hommel, F. J. Bryant, M. J. R. Swift, W. Busse, und H. E. Gumlich, The Influence of II-VI Lattice Damage on Rare Earth Sites as Determined by Site Selective Spectroscopy and Cathodoluminescence: Argon Implanted ZnS:Sm${ }^{3+}$, J. Lumin. 52, 325 (1992).

[Hon03] W. K. Hong, J. I. Sohn, D. K. Hwang, S. S. Kwon, G. Jo, S. Song, S. M. Kim, H. J. Ho, S. J. Park, M. E. Welland, und T. Lee, Tunable Electronic Transport Characteristic of Surface-Architecture-Controlled ZnO Nanowire Field Effect Transistors, Nano Lett. 8, 950 (2003).

[Hua01a] M. H. Huang, S. Mao, H. Feick, H. Yan, Y. Wu, H. Kind, E. Weber, R, Russo, und P. Yang, Room-Temperature Ultraviolet Nanowire Nanolasers, Science 292, 1897 (2001).

[Hua01b] Y. Huang, X. Duan, Y. Cui, L. J. Lauhon, K. H. Kim, und C. M. Lieber, Logic Gates and Computation from Assembled Nanowire Building Blocks, Science 294, 1313 (2001).

[Hva83] J. M. Hvam, G. Blattner, M. Reuscher, und C. Klingshirn, The Biexciton Levels and Nonlinear Optical Transitions in ZnO, Phys. Stat. Sol. B 118, 176 (1983).

[Iij91] S. Iijima, Helical Microtubules of Graphitic Carbon, Nature 354, 56 (1991).

[Ip06] K. Ip, G. T. Thaler, H. Yang, S. Y. Han, Y. Li, D. P. Norton, S. J. Pearton, S. Jang, und F. Ren, Contacts to ZnO, J. Cryst. Growth 287, 149 (2006).

[Jav02] A. Javey, H. Kim, Q. Wang, A. Ural, J. Guo, P. Mcintyre, P. Mceuen, M. Lundstrom, und H. Dai, High- $\kappa$ Dielectrics for Advanced CarbonNanotube Transistors and Logic Gates, Nature Materials 1, 241 (2002). 
[Jia03] Y. Jiang, X. M. Meng, C. S. Lee, und S. T. Lee, ZnS Nanowires with Wurtzite Polytype Modulated Structure, Adv. Mater. 15, 1195 (2003).

[Joa00] C. Joachim, J. K. Gimzewski, und A. Aviram, Electronics Using HybridMolecular and Mono-Molecular Devices, Nature 408, 541 (2000).

[Joh02] J. C. Johnson, H. J. Choi, K. P. Knutsen, R. D. Schaller, P. Yang, und R. J. Saykally, Single Gallium Nitride Nanowire Lasers, Nature Materials 1, 106 (2002).

[Joh03] J. C. Johnson, H. Yan, P. Yang, und R. J. Saykally, Optical Cavity Effects in ZnO Nanowire Lasers and Waveguides, J. Phys. Chem. B 107, 8816 (2003).

[Joh06] J. Johansson, L. S. Karlsson, C. P. T. Svensson, T. Martensson, B. A. Wacaser, K. Deppert, L. Samuelson, und W. Seifert, Structural Properties of $\langle 111\rangle$ B-Oriented III-V Nanowires, Nature Materials 5, 574 (2006).

[Jon88] K. S. Jones, S. Prussin, und E. R. Weber, A Systematic Analysis of Defects in Ion Implanted Silicon, Appl. Phys. A 45, 1 (1988).

[Ju05] S. Ju, K. Lee, D. B. Janes, M. H. Yoon, A. Facchetti, und T. J. Marks, Low Operating Voltage Single ZnO Nanowire Field-Effect Transistors Enabled by Self-Assembled Organic Gate Dielectrics, Nano Lett. 5, 2281 (2005).

[Ju06] S. Ju, K. Lee, D. B. Janes, R. C. Dwivedi, H. Baffour-Awuah, R. Wilkins, M. H. Yoon, A. Facchetti, und T. J. Mark, Proton Radiation Hardness of Single-Nanowire Transistors Using Robust Organic Gate Nanodielectrics, Appl. Phys. Lett. 89, 073510 (2006).

[Ju07] S. Ju, K. Lee, M. H. Yoon, A. Facchetti, T. J. Marks, und D. B. Janes, High Performance ZnO Nanowire Field Effect Transistor with Organic Gate Nanodielectrics: Effect of Metal Contacts and Ozone Treatment, Nanotechnology 18, 155201 (2007).

[Kam01] T. I. Kamins, R. S. Williams, D. P. Basile, T. Hesjedal, und J. S. Harris, Ti-Catalyzed Si Nanowires by Chemical Vapor Deposition: Microscopy and Growth Mechanism, J. Appl. Phys. 89, 1008 (2001).

[Kam03] T. I. Kamins, X. Li, und R. S. Williams, Growth and Structure of Chimically Vapor Deposited Ge Nanowires on Si Substrates, Nano Lett. 4, 503 (2003). 
[Kay88] Y. Kayanuma, Quantum-Size Effects of Interacting Electrons and Holes in Semiconductor Microcrystals with Spherical Shape, Phys. Rev. B 38, 9797 (1988).

[Ken95] T. A. Kennedy, E. R. Glaser, P. B. Klein, und R. N. Bhargava, Symmetry and Electronic Structure of the Mn Impurity in ZnS Nanocrystals, Phys. Rev. B 52, R14356 (1995).

[Kim07a] H. B. Kim, G. Hobler, A. Steiger, A. Lugstein, und E. Bertagnolli, Full Three-Dimensional Simulation of Focused Ion Beam Micro/nanofabrication, Nanotechnology 18, 245303 (2007).

[Kim07b] H. B. Kim, G. Hobler, A. Steiger, A. Lugstein, und E. Bertagnolli, Level Set Approach for the Simulation of Focused Ion Beam Processing on the Micro/nano Scale, Nanotechnology 18, 265307 (2007).

[Kla04] P. J. Klar, L. Chen, W. Heimbrodt, F. J. Brieler, M. Fröba, T. Kurz, H. A. Krug von Nidda, und A. Loidl, Modified Magnetic Properties of Paramagnetic (Zn, Mn)S at Reduced Dimensions, Adv. in Solid State Phys. 44, 491 (2004).

[Kle97] D. L. Klein, R. Roth, A. K. L. Lim, A. P. Alivisatos, und P. L. McEuen, A Single-Electron Transistor Made from a Cadmium Selenide Nanocrystal, Nature 389, 699 (1997).

[Kli75] C. Klingshirn, The Luminescence of $\mathrm{ZnO}$ under High One- and TwoQuantum Excitation, Phys. Stat. Sol. B 71, 547 (1975).

[Kli05] C. Klingshirn, Semiconductor Optics, Springer Verlag Berlin Heidelberg, 2 Aufl. (2005).

[Kli07] C. Klingshirn, ZnO: From Basics towards Applications, Phys. Stat. Sol. B 244, 3027 (2007).

[Kör88] W. Körber und A. Hangleiter, Excitation and Decay Mechanism of the Intra-4f Luminescence of $\mathrm{Yb}^{3+}$ in Epitaxial InP:Yb Layers, Appl. Phys. Lett. 52, 114 (1988).

[Kuc03] S. O. Kucheyev, C. Jagadish, J. S. Williams, P. N. K. Deenapanray, M. Yano, K. Koike, S. Sasa, M. Inoue, und K. Ogata, Implant Isolation of ZnO, J. Appl. Phys. 93, 2972 (2003). 
[Kul80] S. S. Kular, B. J. Sealy, K. G. Stephens, D. Sadana, und G. R. Booker, Electrical, Rutherford Backscatttering and Transmission Electron Microscopy Studies of Furnace Annealed Zinc Implanted GaAs, Solid State Electron. 23, 831 (1980).

[LaB99] "ZnO" in II-VI and I-VII Compounds, Bd. 41 von Landolt-Börnstein, Springer, Heidelberg (1999), supplement to III/17b chap. Semiconductors.

[Lat99] C. D. Latham, M. Haugk, R. Jones, Th. Frauenheim, und P. R. Briddon, Density-Functional Calculations of Carbon Diffusion in GaAs, Phys. Rev. $B$ 60, 15117 (1999).

[Lau02] L. J. Lauhon, M. S. Gudiksen, D. Wang, und C. M. Lieber, Epitaxial Core-Shell and Core-Multishell Nanowire Heterostructures, Nature 420, 57 (2002).

[Law05] M. Law, L. E. Greene, J. C. Johnson, R. Saykally, und P. Yang, Nanowire Dye-Sensitized Solar Cells, Nature Materials 4, 455 (2005).

[Lee00] N. S. Lee, D. S. Chung, J. H. Kang, H. Y. Kim, S. H. Park, Y. W. Jin, Y. S. Choi, I. T. Han, N. S. Park, M. J. Yun, J. E. Jung, C. J. Lee, J. H. You, S. H. Jo, C. G. Lee, und J. M. Kim, Carbon Nanotube-Based FieldEmission Displays for Large-Area and Full-Color Applications, Jpn. J. Appl. Phys. 39, 7154 (2000).

[Lei01] F. H. Leiter, H. R. Alves, A. Hofstaetter, D. M. Hoffmann, und B. K. Meyer, The Oxygen Vacancy as the Origin of a Green Emission in Undoped ZnO, Phys. Stat. Sol. B 226, R4 (2001).

[Li04] D. Li und Y. Xia, Electrospinning of Nanofibers: Reinventing the Wheel?, Adv. Mater. 16, 1151 (2004).

[Lie01] C. M. Lieber, The Incerdible Shrinking Circuit, Scientific American 285, 58 (2001).

[Lin06] K. F. Lin, H. M. Cheng, H. C. Hsu, und W. F. Hsieh, Band Gap Engineering and Spatial Confinement of Optical Phonon in ZnO Quantum Dots, Appl. Phys. Lett. 88, 263117 (2006).

[Lio98] H. C. Liou und J. Pretzer, Effect of Curing Temperature on the Mechanical Properties of Hydrogen Silsesquioxane Thin Films, Thin Solid Films 335, 186 (1998). 
[Liu07] Y. Liu, W. Luo, R. Li, und X. Chen, Spectroscopic Evidence of MultipleSite Structure of $\mathrm{Eu}^{3+}$ Ions Incorpoated in ZnO Nanocrystals, Optics Letters 32, 566 (2007).

[Liu08] Y. Liu, W. Luo, R. Li, G. Liu, M. R. Antonio, und X. Chen, Optical Spectroscopy of Eu ${ }^{3+}$ Doped ZnO Nanocrystals, J. Chem. Phys. C 112, 686 (2008).

[Loo99] D. C. Look, J. W. Hemsky, und J. R. Sizelove, Residual Native Shallow Donor in ZnO, Phys. Rev. Lett. 82, 2552 (1999).

[Loo02] D. C. Look, D. C. Reynolds, C. W. Litton, R. L. Jones, D. B. Eason, und G. Cantwell, Characterization of Homoepitaxial p-Type ZnO Grown by Molecular Beam Epitaxy, Appl. Phys. Lett. 81, 1830 (2002).

[Loo04] D. C. Look und B. Clafin, P-Type Doping and Devices Based on ZnO, Phys. Stat. Sol. B 241, 624 (2004).

[Lor05] K. Lorenz, E. Alves, E. Wendler, O. Bilani, W. Wesch, und M. Hayes, Damage Formation and Annealing at Low Temperatures in Ion Implanted ZnO, Appl. Phys. Lett. 87, 191904 (2005).

[Lu07] J. Lu, Q. Liang, Y. Zhang, Z Ye, und S. Fujita, Improved p-Type Conductivity and Acceptor States in N-Doped ZnO Thin Films, J. Phys. D: Appl. Phys 40, 3177 (2007).

[Luc02] A. Lucca, D. W. Hamby, M. J. Klopfstein, und G. Cantwell, Chemomechanical Polishing Effects on the Room Temperature Photoluminescence of Bulk ZnO: Exciton-LO Phonon Interaction, Phys. Stat. Sol. B 229, 845 (2002).

[Lup99] A. Lupei, V. Lupei, C. Presura, V. N. Enaki, und A. Petraru, ElectronPhonon Coupling Effects on $Y^{3+}$ Spectra in Several Laser Crystals, J. Phys.: Condens. Matter 11, 3769 (1999).

[Ma03] C. Ma, D. Moore, J. Li, und Z. L. Wang, Nanobelts, Nanocombs, and Nanowindmills of Wurtzite ZnS, Adv. Mater. 15, 228 (2003).

[Mäd02] L. Mädler, W. J. Stark, und S. E. Pratsinis, Rapid Synthesis of Stable ZnO Quantum Dots, J. Appl. Phys. 92, 6537 (2002).

[Mak01] T. Makino, Y. Segawa, M. Kawasaki, A. Ohtomo, R. Shiroki, K. Tamura, T. Yasuda, und H. Koinuma, Band Gap Engineering Based on 
$\mathrm{Mg}_{x} Z n_{1-x} \mathrm{O}$ and $C d_{y} Z n_{1-y} O$ Ternary Alloy Films, Appl. Phys. Lett. 78, 1237 (2001).

[Mak03] A. V. Makarov, S. G. Zbezhneva, V. V. Kovalenko, und M. N. Rumyantseva, Mass Spectrometric Study of Nanocrystalline ZnO Vaporization, Inorg. Mater 39, 594 (2003).

[Mar98] R. Martel, T. Schmidt, H. R. Shea, T. Hertel, und Ph. Avouris, Singleand Multi-Wall Carbon Nanotube Field-Effect Transistors, Appl. Phys. Lett. 73, 2447 (1998).

[May70] J. W. Mayer, L. Eriksson, und J. A. Davies, Ion Implantation in Semiconductors: Silicon and Germanium, New York: Acad. Press (1970).

[McC95] D. G. McCulloch und S. Prawer, The Effect of Annealing and Implantation Temperature on the Structure of C Ion-Beam-Irradiated Glassy Carbon, J. Appl. Phys. 78, 3040 (1995).

[Mei78] L. G. Meiners, Surface Potential of Anodized p-GaAs MOS Capacitors, Appl. Phys. Lett. 33, 747 (1978).

[Mei01] J. D. Meindl, Q. Chen, und J. A. Davis, Limits on Silicon Nanoelectronics for Terascale Integration, Science 293, 2044 (2001).

[Mey04] B. K. Meyer, K. Alves, D. M. Hoffmann, W. Kriegseis, D. Forster, F. Bertram, J. Christen, A. Hoffmann, M. Strassburg, M. Dworzak, U. Haboeck, und A. V. Rodina, Bound Exciton and Donor - Acceptor Pair Recombinations in ZnO, Phys. Stat. Sol. B 241, 231 (2004).

[Mey05] B. K. Meyer, J. Sann, D. M. Hoffmann, und A. Zeuner, Shallow Donors and Acceptors in ZnO, Semicond. Sci. Technol. 20, 62 (2005).

[Mil06] S. Milenkovic, A. W. Hassel, und A. Schneider, Effect of the Growth Conditions on the Spatial Features of Re Nanowires Produced by Directional Solidification, Nano Lett. 6, 794 (2006).

[Moe02] W. Moeller und M. Posselt, TRIDYN (2002), Forschungszentrum Rossendorf, Dresden.

[Mon04] T. Monteiro, M. J. Soares, A. Neves, M. Oliveira, E. Rita, U. Wahl, und E. Alves, Optical and RBS Studies in Tm Implanted ZnO Samples, Phys. Stat. Sol. C 1, 254 (2004). 
[Mon06] T. Monteiro, M. J. Soares, A. Neves, S. Pereira, M. R. Correia, M. Peres, E. Alves, D. Rogers, F. Teherani, V. Munzo-SanJose, T. Trindade, und A. Pereira, Optical Active Centres in ZnO Samples, J. Non-Cryst. Sol. 352, 1453 (2006).

[Moo65] G. E. Moore, Cramming More Components onto Integrated Circuits, Electronics 38, 114 (1965).

[Mot07] A. Motayed, A. V. Davydov, M. He, S. N. Mohammad, und J. Meingailis, 365 nm Operation of n-Nanowire/p-Gallium Nitride Homojunction Light Emitting Diodes, Appl. Phys. Lett. 90, 183120 (2007).

[Mue00] G. Mueller, Electroluminescence II, Academic Press (2000).

[Mü105] S. Müller, Wachstum von ZnO Nanodrähten und deren Dotierung durch Ionenstrahlen, Diplomarbeit (2005).

[Mül07] S. Müller, D. Stichtenoth, M. Uhrmacher, H. Hofsäss, C. Ronning, und J. Röder, Unambiguous Identification of the PL-I9 Line in Zinc Oxide, Appl. Phys. Lett. 90, 012107 (2007).

[Mut99] J. F. Muth, R. M. Kolbas, A. K. Sharma, S. Oktyabrsky, und J. Narayan, Excitonic Structure and Absorption Coefficient Measurements of ZnO Single Crystal Epitaxial Films Deposited by Pulsed Laser Deposition, J. Appl. Phys. 85, 7884 (1999).

[Nag75] H. M. Naguib und R. Kelly, Criteria for Bomabardment-Induced Structural Changes in Non-Metallic Sollids, Radiation Effects 25, 1 (1975).

[Nye03] E. E. Nyein, U. Hömmerich, J. Heikenfeld, D. S. Lee, A. J. Steckl, und J. M. Zavada, Spectral and Time-Resolved Photoluminescence Studies of Eu-Doped GaN, Appl. Phys. Lett. 82, 1655 (2003).

[Odo98] T. Odom, J. L. Huang, P. Kim, und C. M. Lieber, Atomic Structure and Electronic Properties of Single-Walled Carbon Nanotubes, Nature 391, 62 (1998).

[Ohl02] B. J. Ohlsson, M. T. Björk, A. I. Persson, C. Thelander, L. R. Wallenberg, M. H. Magnusson, K. Deppert, und L. Samuelson, Growth and Characterization of GaAs and InAs Nano-Whiskers and InAs/GaAs Heterostructures, Physica E 13, 1126 (2002). 
[Oht98] A. Ohtomo, M. Kawasaki, T. Koida, K. Masubuchi, H. Koinuma, Y. Sakurai, Y. Yoshida, T. Yasuda, und Y. Segawa, $M g_{x} Z n_{1-x} O$ as a II-IV Widegap Semiconductor Alloy, Appl. Phys. Lett. 72, 2466 (1998).

[Oka90] H. Okamoto und T. B. Massalski, Binary Alloy Phase Diagrams, Material Park, OH (1990).

[Özg05] Ü. Özgür, Ya. I. Alivov, C. Liu, A. Teke, M. A. Reshchikov, S. Dogan, V. Avrutin, S. J. Cho, und H. Morkoc, A Comprehensive Review of ZnO Materials and Devices, J. Appl. Phys. 98, 041301 (2005).

[Pal97] C. Palacios, M. A. Parada, und A. Lahsen, Upper Jurassic Au-Zn Mineralization in El Faldeo District, Chilean Patagonia, Geol. Rundsch 86, 132 (1997).

[Par67] Y. S. Park und D. C. Reynolds, Growth of ZnO Single Crystals, J. Appl. Phys. 53, 756 (1967).

[Par02] C. H. Park, S. B. Zhang, und S. H. Wei, Origin of p-Type Doping Difficulty in ZnO: The Impurity Perspective, Phys. Rev. B 66, 073202 (2002).

[Par04a] W. I. Park, J. S. Kim, G. C. Yi, M. H. Bae, und H. J. Lee, Fabrication and Electric Charcteristics of High-Performance ZnO Nanorod Field-Effect Transistors, Appl. Phys. Lett. 85, 5052 (2004).

[Par04b] W. I. Park und G. C. Yi, Electroluminescence in n-ZnO Nanorod Arrays Vertically Grown on p-GaN, Adv. Mater. 16, 87 (2004).

[Pat06] F. Patolsky, G. Zheng, und C. M. Lieber, Nanowire Sensors for Medicine and the Life Sciences, Nanomedicine 1, 51 (2006).

[Pee00] P. S. Peercy, The Drive to Miniaturization, Nature 406, 1023 (2000).

[Per82] A. Permogorov, Excitons, Bd. 2, S. 177, North-Holland, Amsterdam (1982).

[Per04] A. I. Persson, W. L. Larsson, S. Stenström, B. J. Ohlsson, L. Samuelson, und L. R. Wallenberg, Solid-Phase Diffusion Mechanism for GaAs Nanowire Growth, Nature Materials 3, 677 (2004).

[Per07] M. Peres, A. Cruz, S. Pereira, M. R. Correia, M. J. Soares, A. Neves, M. C. Carmo, T. Monteiro, A. S. Pereira, M. A. Martins, T. Trindade, E. Alves, S. S. Nobre, und R. A. Sa Ferreira, Optical Studies of ZnO Nanocrystals Doped with Eu ${ }^{3+}$ Ions, Appl. Phys. A 88, 129 (2007). 
[Pri04] H. Priller, J. Bückner, Th. Gruber, C. Klingshirn, H. Kalt, A. Waag, H. J. Ko, und T. Yao, Comparison of Linear and Nonlinear Optical Spectra of Various ZnO Epitaxial Layers and of Bulk Material Obtained by Different Experimental Techniques, Phys. Stat. Sol. B 241, 587 (2004).

[Pro00] R. Provoost, Magneto-Photoluminescence of Stacked Quantum Dots and Stress Analysis of Meltgrown Superconductors by Raman Spectroscopy, Dissertation (2000).

[Pro07] W. Prost, K. Blecker, Q. T. Do, I. Regolin, D. Stichtenoth, S. Müller, K. Wegener, C. Ronning, und F. J. Tegude, Modeling the Carrier Mobility in Nanowire Channel FET, in M. Shim, M. Kuno, X. Lin, R. Pachter, und S. Kuma (eds.), Low-Dimensional Materials - Synthesis, Assembly, Property Scaling, and Modeling, Bd. 1017, pp. DD14-06 (2007).

[Rau99] E. Rauly, O. Potavin, F. Balestra, und C. Raynaud, On the Subthreshold Swing and Short Channel Effects in Single and Double Gate Deep Submicron SOI-MOSFETs, Solid State Electron. 43, 2033 (1999).

[Ree00] M. A. Reed und J. A. Tour, Computing with Molecules, Sci. Am. 282, 86 (2000).

[Rei98] L. Reimer, Scanning Electron Microscopy: Physics of Image Formation and Microanalysis, Optical Science, Springer, Berlin (u.a.) (1998).

[Rid98] M. C. Ridgway, C. J. Glover, G. J. Foran, und K. M. Yu, Characterization of the Local Structure of Amorphous GaAs Produced by Ion Implantation, J. Appl. Phys. 83, 4610 (1998).

[Rit03] E. Rita, E. Alves, U. Wahl, J. G. Correia, A. J. Neves, M. J. Soares, und T. Monteiro, Optical Doping of $\mathrm{ZnO}$ with Tm by Ion Implantation, Physica B 340, 235 (2003).

[Rit06] E. Rita, E. Alves, U. Wahl, J. G. Correia, T. Monteiro, M. J. Soares, A. Neves, und M. Peres, Stability and Luminescence Studies of Tm and Er Implanted ZnO Single Crystals, Nucl. Instrum. Meth. B 242, 580 (2006).

[Roc05] M. C. Roco, National Nanotechnology Initiative at Five Years: From Concept to Reality (2005), NSF, Discovery Lecture Series.

[Rom03] J. F. Rommeluère, L. Svob, F. Jomard, J. Mimila-Arooyo, V. Lusson, A. Nd Sallet, und Y. Marfaing, Electrical Activity of Nitrogen Acceptors 
in ZnO Films Grown by Metalorganic Vapor Phase Epitaxy, Appl. Phys. Lett. 83, 287 (2003).

[Ros05] F. M. Ross, J. Tersoff, und M. C. Reuter, Sawtooth Facetting in Silicon Nanowires 95, $1461 ß 4$ (2005).

[Sam04] L. Samuelson, B. J. Ohlsson, M. T. Björk, und H. Xu, Nanowires and Nanobelts - Materials, Properties and Devices, chap. 3, Kluwer Academic Publishers (2004).

[San96] C. J. Sansonetti, M. L. Salit, und J. Reader, Wavelengths of Spectral Lines in Mercury Pencil Lamps, Applied Optics 35, 74 (1996).

[Sat04] K. Sato, T. Takasmasu, und G. Kido, Photoluminescence Study of Low Temperature Epitaxial Growth Yb-Doped $A l_{x} G a_{1-x} A s$, J. Appl. Phys. 95, $2924(2004)$.

[Sch53] E. Scharowsky, Optische und Elektrische Eigenschaften von ZnOEinkristallen mit Zn-Überschuß, Zeitschrift für Physik 135, 318 (1953).

[Sch04] L. Schubert, P. Werner, N. D. Zakharov, G. Gerth, F. M. Kolb, L. Long, und U. Gösele, Silicon Nanowhiskers Grown on 111 Si Substrates by Molecular-Beam Epitaxy, Appl. Phys. Lett. 84, 24 (2004).

[Sch06a] K. F. Schmidt, Nanofrontiers - Visions for the Future of Nanotechnology, Woodrow Wilson International Center for Scholars, Washington DC (2006).

[Sch06b] M. Schulenburg, Nanotechnologie - Innovation für die Welt von Morgen, Bundesministerium für Bildung und Forschung, Berlin (2006).

[Sen03] R. T. Senger und K. K. Bajaj, Optical Properties of Confined Polaronic Excitons in Spherical Ionic Quantum Dots, Phys. Rev. B 68, 045313 (2003).

[Sha04] I. Shalish, H. Temkin, und V. Narayanamurti, Size-Dependent Surface Luminescence in ZnO Nanowires, Phys. Rev. B 69, 245401 (2004).

[Sha06] M. A. Shannon, P. W. Bohn, M. Elimelech, J. G. Georgiadis, B. J. Marinas, und A. M. Mayes, Science and Technology for Water Purification in the Coming Decades, Nature 452, 301 (2006). 
[Shi01] W. S. Shi, Y. F. Zheng, N. Wang, C. S. Lee, und S. T. Lee, A General Synthetic Route to III-V Compound Semiconductor Nanowires, Adv. Mater. 8, 591 (2001).

[Sho52] W. Shockley, A Unipolar Field-Effect Transistor, Proc. IRE 40, 1365 (1952).

[Sig69] P. Sigmund, Theory of Sputtering. I. Sputtering Yield of Amorphous and Polycrystalline Targets, Phys. Rev. B 184, 383 (1969).

[Smi00] B. A. Smith, J. Z. Zhang, A. Joly, und J. Liu, Luminescence Decay Kinetics of $\mathrm{Mn}^{2+}$-Doped $\mathrm{ZnS}$ Nanoclusters Grown in Reverse Micelles, Phys. Rev. B 62, 2021 (2000).

[Sri04] A. Srivastava, O. N. Srivastava, S. Talapatra, R. Vajtai, und P. M. Ajayan, Carbon Nanotube Filters, Nature Materials 3, 610 (2004).

[Sti05] D. Stichtenoth, Wachstum und Modifikation von Halbleiternanodrähten, Diplomarbeit (2005).

[Sti06a] D. Stichtenoth, Growth and Properties of Zincsulfide Nanowires, in Functional Properties of Nanostructured Materials, NATO-ASI, S. 407, Springer (2006).

[Sti06b] D. Stichtenoth, D. Schwen, S. Müller, C. Borchers, und C. Ronning, Optical Activation of Implanted Impurities in ZnS Nanowires, J. Vac. Sci. Technol. A 24, 1356 (2006).

[Sti07] D. Stichtenoth, C. Ronning, T. Niermann, L. Wischmeier, C. J. Chien, P. C. Chang, und J. G. Lu, Optical Size Effects in Ultrathin ZnO Nanowires, Nanotechnology 2007, 435701 (2007).

[Sti08a] D. Stichtenoth, J. Dürr, C. Ronning, L. Wischmeier, und T. Voss, Characterization of the Donor-Acceptor-Pair Transition in Nitrogen Implanted Zinc Oxide, J. Appl. Phys. 103, 083513 (2008).

[Sti08b] D. Stichtenoth, K. Wegener, C. Gutsche, I. Regolin, F. J. Tegude, W. Prost, und C. Ronning, P-Type Doping of GaAs Nanowires, Appl. Phys. Lett. 92, 163107 (2008).

[Stö00] Arno Stötzler, Identifikation und Charakterisierung von Defektzuständen in GaN durch Radioaktive Isotope, Dissertation (2000). 
[Sun01] H. D. Sun, T. Makino, N. T. Tuan, Y. Segawa, M. Kawasaki, A. Ohtomo, K. Tamura, und H. Koinuma, Temperature Dependence of Excitonic Absorption Spectra in $\mathrm{ZnO} / \mathrm{Zn}_{0.88} \mathrm{Mg}_{0.12} \mathrm{O}$ Multiquantum Wells Grown on Lattice-Matched Substrates, Appl. Phys. Lett. 78, 2464 (2001).

[Sun06] H. Sun, Q. F. Zhang, und J. L. Wu, Electroluminescence from ZnO Nanorods with an $n-Z n O / p$-Si Heterojunction Structure, Nanotechnology 17, 2271 (2006).

[Swi89] K. Swiatek, M. Godlewski, D. Hommel, und H. Hartmann, On the Incorporation of Rare Earth Ions Into II-VI Compounds: ZnS:Eu, Phys. Stat. Sol. a 114, 127 (1989).

[Sze81] S. M. Sze, Physics of Semiconductor Devices, Wiley, New York, 2 Aufl. (1981).

[Tek04] A. Teke, Ü. Özgür, S. Dogan, X. Gu, H. Morkoc, B. Nemeth, J. Nause, und H. O. Everitt, Excitonic Fine Structure and Recombination Dynamics in Single-Crystalline ZnO, Phys. Rev. B 70, 195207 (2004).

[Tho60] D. G. Thomas, The Exciton Spectrum of Zinc Oxide, J. Phys. Chem. Solids 15, 86 (1960).

[Tho65] D. G. Thomas, J. J. Hopfield, und W. M. Augustyniak, Kinetics of Radiative Recombination at Randomly Distributed Donors and Acceptors, Phys. Rev 140, A202 (1965).

[Tho01] K. Thonke, Th. Gruber, N. Teofilov, R. Schönfelder, A. Waag, und R. Sauer, Donor-acceptor Pair Transitions in ZnO Substrate Material, Physica B 308, 945 (2001).

[Tra90] V. V. Travnikov, A. Freiberg, und S. F. Savikhin, Surface Excitons in ZnO Crystals, J. Lumin. 47, 107 (1990).

[Tsi87] Y. P. Tsividis, Operation and Modeling of the MOS Transistor, McGrawHill, New York (1987).

[Tsu05] A. Tsukazaki, A. Ohtomo, T. Onuma, M. Ohtani, T. Makino, M. Sumiya, K. Ohtani, S. F. Chichibu, S. Fuke, Y. Segawa, H. Ohno, H. Koinuma, und M. Kawasaki, Repeated Temperature Modulation Epitaxy for p-Type Doping and Light-Emitting Diode Based on ZnO, Nature Materials 4, 42 (2005). 
[Uhr05] M. Uhrmacher und H. Hofsäss, Ion Accelerator Facilities at the University of Göttingen 240, 48 (2005).

[Var67] Y. P. Varshni, Temperature Dependence of the Energy Gap in Semiconductors, Physica 34, 149 (1967).

[Vec80] J. A. van Vechten, Handbook of Semiconductors, Materials and Preparation, North-Holland, Amsterdam (1980), vol. 3, Kap. 1.

[Vij98] D. R. Vij (ed.), Luminescence of Solids, Plenum Publishing Corporation (1998).

[Vis03] R. Viswanatha, S. Sapra, B. Satpati, P. V. Satyam, B. N. Dev, und D. D. Sarma, Understanding the Quantum Size Effect in ZnO Nanocrystals, J. Mater. Chem. 14, 661 (2003).

[Vle37] J. H. van Vleck, The Puzzle of Rare-Earth Spectra in Solids, J. Phys. Chem. 41, 67 (1937).

[Vos06] T. Voss, C. Bekeny, L. Wischmeier, H. Gafsi, S. Börner, W. Schade, A. C. Mofor, A. Bakin, und A. Waag, Influence of Exciton-Phonon Coupling on the Energy Position of the Near-Band-Edge Photoluminescence of ZnO Nanowires 89, 182107 (2006).

[Vos08] T. Voss und L. Wischmeier, Recombination Dynamics of Surface-Related Excitonic States in Single ZnO Nanowires, J. Nanosci. Nanotechnol. 8, 228 (2008).

[Wac06] B. A. Wacaser, K. Deppert, L. S. Karlsson, L. Samuelson, und W. Seifert, Growth and Characterization of Defect Free GaAs Nanowires, J. Cryst. Growth 287, 504 (2006).

[Wag64] R. S. Wagner und W. C. Ellis, Vapor-Liquid-Solid Mechanism of Single Crystal Growth, Appl. Phys. Lett. 4, 89 (1964).

[Wag70] R. S. Wagner, Whisker Technology, Wiley, New York (1970).

[Wal00] C. G. Van de Walle, Hydrogen as a Cause of Doping in Zinc Oxide, Phys. Rev. Lett. 85, 1012 (2000).

[Wan00] Z. L. Wang, Transmission Electron Microscopy of Shape-Controlled Nanocrystals and their Assemblies, J. Phys. Chem. B 104, 1153 (2000). 
[Wan03] Z. L. Wang, New Developments in Transmission Electron Microscopy for Nanotechnology, Adv. Mater. 15, 1497 (2003).

[Wan04a] X. Wang, Y. Ding, C. J. Summers, und Z. L. Wang, Large-Scale Synthesis of Six-Nanometer-Wide ZnO Nanobelts, J. Phys. Chem. B 108, 8733 (2004).

[Wan04b] Z. L. Wang (ed.), Nanowires and Nanobelts - Materials, Properties and Devices, Bd. 1, Kluwer Academic Publishers, Dordrecht, The Netherlands (2004).

[Wan06] J. W. Wang, M. J. Zhou, S. K. Hark, Q. Li, D. Tang, M. W. Chu, und C. H. Chen, Local Electronic Structure and Luminescence Properties of Er Doped ZnO Nanowires, Appl. Phys. Lett. 89, 221917 (2006).

[Wes92] W. Wesch, Ion Implantation in III-V Compounds, Nucl. Instrum. Meth. B 68, 342 (1992).

[Wes97] J. Westwater, D. P. Gosian, S. Tomiya, S. Usui, und H. Ruda, Growth of Silicon Nanowires via Gold/silane Vapor-liquid-solid Reaction, J. Vac. Sci. Technol. B 15, 554 (1997).

[Win02] S. J. Wind, J. Appenzeller, R. Martel, V. Derycke, und Ph. Avouris, Vertical Scaling of Carbon Nanotube Field-Effect Transistors Using Top Gate Electrodes, Appl. Phys. Lett. 80, 3817 (2002).

[Wis06a] L. Wischmeier, T. Voss, S. Börner, und W. Schade, Comparison of the Optical Properties of as-Grown Ensembles and Single ZnO Nanowires, Appl. Phys. A 84, 111 (2006).

[Wis06b] L. Wischmeier, T. Voss, I. Rückmann, J. Gutowski, A. C. Mofor, A. Bakin, und A. Waag, Dynamics of Surface-Exciton Emission in ZnO Nanowires, Phys. Rev. B 74, 195333 (2006).

[Wis07] L. Wischmeier, ZnO-Nanodrähte: Optische Eigenschaften und Ladungsträgerdynamik, Dissertation (2007).

[Wu00] Y. Wu und P. Yang, Germanium Nanowire Growth via Simple Vapor Transport, Chem. Mater. 12, 605 (2000).

[Wu01] X. C. Wu, W. H. Song, K. Y. Wang, T. Hu, B. Zhao, Y. P. Sun, und J. J. $\mathrm{Du}$, Preparation and Photoluminescence Properties of Amorphous Silica Nanowires, Chem. Phys. Lett. 336, 53 (2001). 
[Wün80] W. Wünstel und C. Klingshirn, Tunable Laser-Emission from WurtziteType II-VI Compounds, Optics Communications 32, 269 (1980).

[Xia03] Y. Xia, P. Yang, Y. Sun, Y. Wu, B. Mayers, B. Gates, Y. Yin, F. Kim, und H. Yan, One-Dimensional Nanostructures: Synthesis, Characterization, and Applications, Adv. Mater. 15, 353 (2003).

[Xio05] G. Xiong, K. B. Ucer, R. T. Williams, J. Lee, D. Bhattacharyya, J. Metson, und P. Evans, Donor-Acceptor Pair Luminescence of NitrogenImplanted ZnO Single Crystal, J. Appl. Phys. 97, 043528 (2005).

[Yag06] P. Yager, T. Edwards, E. Fu, K. Helton, K. Nelson, M. R. Tam, und B. H. Weigl, Microfluidic Diagnostic Technologies for Global Public Health, Nature 442, 412 (2006).

[Yam01] A. Yamamoto, K. Miyajima, T. Goto, H. J. Ko, und T. Yao, Biexciton Luminescence in High-Quality ZnO Epitaxial Thin Films, J. Appl. Phys. 90, 4973 (2001).

[Yan98] K. Yan, C. Duan, Y. Ma, S. Xia, und J. C. Krupa, Photoluminescence Lifetime of Nanocrystalline ZnS:Mn ${ }^{2+}$, Phys. Rev. B 58, 13585 (1998).

[Yan06] W. Q. Yang, H. B. Huo, L. Dai, R. M. Ma, S. F. Liu, G. Z. Ran, B. Shen, C. L. Lin, und G. G. Qin, Electrical Transport and Electroluminescence Properties of n-ZnO Single Nanowires, Nanotechnology 17, 4868 (2006).

[Yao02] B. D. Yao, Y. F. Chan, und N. Wang, Formation of ZnO Nanostructures by a Simple Way of Thermal Evaporation, Appl. Phys. Lett. 81, 757 (2002).

[Zeu02] A. Zeuner, H. Alves, D. M. Hofmann, B. K. Meyer, A. Hoffmann, U. Haboeck, M. Strassburg, und M. Dworzak, Optical Properties of the Nitrogen Acceptor in Epitaxial ZnO, Phys. Stat. Sol. B 234, R7 (2002).

[Zha03] R. Q. Zhang, Y. Lifshitz, und S. T. Lee, Oxide-Assisted Growth of Semiconductor Nanowires, Adv. Mater. 15, 635 (2003).

[Zha05] L. L. Zhang, C. X. Guo, J. J. Zhao, und J. T. Hu, Photoluminescence of Eu(III)-Doped ZnO Nanopowder and Energy Transfer from ZnO to Eu(III) Ions, Chin. Phys. Lett. 22, 1225 (2005).

[Zie08] J. F. Ziegler, J. P. Biersack, und M. D. Ziegler, SRIM - The Stopping and Ranges of Ions in Matter, Chester, Maryland: CSRIM Co. (2008), see also: www.srim.org. 
[Zim07a] M. A. Zimmler, J. Bao, I. Shalish, W. Yi, V. Narayanamurti, und F. Capasso, A Two-Colour Heterojunction Unipolar Nanowire Light-Emitting Diode by Tunnel Injection, Nanotechnology 18, 395201 (2007).

[Zim07b] M. A. Zimmler, J. Bao, I. Shalish, W. Yi, J. Yoon, V. Narayanamurti, und F. Capasso, Electroluminescence from Single Nanowires by Tunnel Injection: an Experimental Study, Nanotechnology 18, 235205 (2007).

[Zim08] M. A. Zimmler, D. Stichtenoth, C. Ronning, W. Yi, V. Narayanamurti, T. Voss, und F. Capasso, Scalable Fabrication of Nanowire Photonic and Electronic Circuits Using Spin-on Glass, Nano Lett. (2008), in press, doi:10.1021/nl080627w. 


\section{Anhang}

\section{Liste der Publikationen}

Teile dieser Arbeit wurden bereits in nachstehenden Artikeln veröffentlicht:

- C. Ronning, D. Stichtenoth, und S. Müller, „Der Draht zur Welt en miniature", Georgia Augusta 4, 83 (2005).

- D. Stichtenoth, D. Schwen, S. Müller, C. Borchers, und C. Ronning, „Growth and properties of zincsulfide nanowires", in NATO Science Series II, "Functional Properties of Nanostructured Materials", R. Kassing, P. Petkov, W. Kulisch, C. Popov (Eds.) 223, 407 (2006).

- P.C. Chang, Z. Fan, C.J. Chien, D. Stichtenoth, C. Ronning, und J.G. Lu, „High-performance ZnO nanowire field effect transistors“, Appl. Phys. Lett. 89, $133113(2006)$.

- C. Borchers, D. Stichtenoth, S. Müller, D. Schwen, und C. Ronning, „Catalystnanostructure interaction and growth of ZnS nanobelts", Nanotechnology 17, 1067 (2006).

- C. Borchers, S. Müller, D. Stichtenoth, D. Schwen, und C. Ronning, „Catalystnanostructure interaction in the growth of 1-D ZnO nanostructures", J. Phys. Chem. B 110, 1656 (2006).

- D. Stichtenoth, D. Schwen, S. Müller, C. Borchers, und C. Ronning, „Optical activation of implanted impurities in ZnS nanowires", J. Vac. Sci. Technol. A 24, 1356 (2006).

- J. Wang, Q. Li, D. Stichtenoth, S. Müller, und C. Ronning „Nanomaterial electronic structure investigation by valence electron energy loss spectroscopy - An example of doped ZnO nanowires“, Micron (2007) in press, doi:10.1016/j.micron.2007.10.015 
- P.C. Chang, Z. Fan, C.J. Chien, D. Stichtenoth, C. Ronning, und J.G. Lu, „Finite size effect in ZnO nanowires“, Appl. Phys. Lett. 90, 133101 (2006).

- S. Müller, D. Stichtenoth, M. Uhrmacher, H. Hofsäss, und C. Ronning, „Unambiguous identification of the PL-I-9 line in zinc oxide“, Appl. Phys. Lett. 90, 012107 (2007).

- D. Stichtenoth, T. Niermann, P.C. Chang, C.J. Chien, J.G. Lu, L. Wischmeier, T. Voss, und C. Ronning, „Optical size effects in ultrathin ZnO nanowires“, Nanotechnology 18, 435701 (2007).

- L. Chen, T. Niebling, W. Heimbrodt, D. Stichtenoth, C. Ronning, und P.J. Klar, „Dimensional dependence of the dynamics of the Mn 3d5 luminescence in (Zn,Mn)S nanowires and nanobelts“, Phys. Rev. B 76, 115325 (2007).

- W. Prost, K. Blecker, Q.-T. Do, I. Regolin, D. Stichtenoth, S. Müller, K. Wegener, C. Ronning, und F.J. Tegude „Modeling the carrier mobility in nanowire channel FET“, in „Low-dimensional materials - synthesis, assembly, property scaling, and modelling“ M. Shim, M. Kuno, X. Lin, R. Pachter, S. Kuma (eds.)Mat. Res. Soc. Sympo. Proc. 1017, DD14-06 (2007).

- S. Geburt, D. Stichtenoth, S. Müller, W. Dewald, J. Wang, Y. Jiao, Y.Y. Rao, S.K. Hark, Q. Li, und C. Ronning, „Rare earth doped ZnO nanowires“, J. Nanosci. Nanochnol. 8, 244 (2008).

- D. Stichtenoth, J. Dürr, L. Wischmeier, T. Voss, und C. Ronning, „Characterization of the donor-acceptor-pair transition in Nitrogen implanted $\mathrm{ZnO}$ “, J. Appl. Phys. 103, 083513 (2008).

- L. Chen, T. Niebling, W. Heimbrodt, P.J. Klar, D. Stichtenoth, und C. Ronning, „Energy transfer and dynamics of the Mn $3 d^{5}$ luminescence in low dimensional (Zn,Mn)S nanostructures“, Journal of the Korean Physical Society (2008) submitted.

- D. Stichtenoth, K. Wegener, C. Gutsche, I. Regolin, F.J. Tegude, W. Prost, und C. Ronning, „P-type doping of GaAs Nanowires“, Appl. Phys. Lett. 92, 163107 (2008).

- M.A. Zimmler, D. Stichtenoth, C. Ronning, W. Yi, V. Narayanamurti, T. Voss, und F. Capasso „Scalable fabrication of nanowire photonic and electronic circuits using spin-on glass", Nano Lett. (2008) in press, doi:10.1021/nl080627w 


\section{Danke}

An dieser Stelle möchte ich allen Personen danken, die zu dem Gelingen dieser Arbeit beigetragen haben. Allen voran gilt mein Dank Carsten Ronning für die Betreuung dieser Arbeit. Danke, dass ich, als dein erster eigener Doktorand, an dem Thema der Nanodrähte so frei arbeiten durfte. Danke für deine ständige Motivation und unermüdliche Unterstützung, insbesondere dabei die vielen Kollaborationen am Laufen zu halten. Danke dafür, dass du die Teilnahme an den vielen Konferenzen und die Forschungsaufenthalte in Hong Kong ermöglicht hast. Danke, dass deine Tür immer offen stand.

Prof. Hans Hofsäss möchte ich dafür danken, dass er das Nanodrahtprojekt uneingeschränkt unterstützt hat. Ihre unkomplizierte Art und die Motivation zur eigenverantwortlichen Arbeit hat immer für ein positives Arbeitsklima gesorgt.

Frau Prof. Cynthia A. Volkert danke ich für die Bereitschaft diese Arbeit zu begutachten.

Wie sich schon abzeichnete, sind viele der vorgestellten Experimente das Ergebnis einer Vielzahl von Kollaborationen, die im Rahmen dieser Arbeit entstanden sind. Einen sehr guten Rahmen dafür hat das Schwerpunktprogramm 1165 der DFG geboten. Den Kollaborationspartnern im SPP gilt mein aufrichtiger Dank.

Danke Tobias Voss und Lars Wischmeier für die vielen Diskussionen über die Lumineszenz von ZnO - von euch habe ich viel gelernt. Danke, für die netten Messaufenthalte in Bremen und danke Tobias für die nette Sightseeing Tour an der East Coast.

Danke Tobias Niebling, Limei Klar, Prof. Peter J. Klar und Prof. Wolfram Heimbrodt für die wunderbare Zusammenarbeit. Die Messbesuche in Marburg und Diskussionen waren immer sehr motivierend.

Danke Christoph Gutsche und Werner Prost für die sehr nette Zusammenarbeit. Danke insbesondere für die Einführung in die Lithographie, den Standpunkt der Ingenieure und den Aufenthalt in Duisburg. Ich habe gelernt, dass auch das Ruhrgebiet seine Reize hat.

Juan Wang und Prof. Quan Li danke ich für die freundliche Zusammenarbeit und die Gastfreundschaft in Hong Kong sowie für die vielen gemeinsamen TEM-Messungen. Pai-Chun Chang und Prof. Jia Grace Lu gilt mein Dank für die sehr nette und produktive Kollaboration an Zinkoxid-Nanodrähten.

Vielen Dank Mariano Zimmler und Prof. Federico Capasso für die großartige Kollaboration, die netten Gespräche und produktiven Diskussionen in Harvard - das alles war überaus motivieren. 
Für die spontane und unkomplizierte Bereitschaft TEM-Untersuchungen durchzuführen danke ich Tore Niermann, Christine Borchers und PD Michael Seibt.

Außerdem gilt mein Dank natürlich auch den Mitstreitern in der Nanogruppe am zweiten Physikalischen Institut - die irgendwie immer größer wurde: Sven Müller, Wilma Dewald, Katharina Wegener, Sebastian Geburt und Joachim Dürr.

Meine Roomies in der Zeit meiner Doktorarbeit darf ich natürlich nicht vergessen: danke Daniel Schwen für die wissenschaftlichen Diskussionen aber auch für die nette Atmosphäre im Büro, für die Abhängigkeit von Serien und die vielen Kickerspiele. Danke Sören Eyhusen für allzeit offene Ohren und die vielen Wahrheiten. Danke Johannes Bosman, dass du trotz Kaffeefleck auf dem Teppich in unser Büro gezogen bist.

Dank gilt darüber hinaus der ganzen Gruppe Hofsäss/Ronning für spontane Diskussionen und Kaffeepausen, Hilfe bei Messungen und die gemeinsamen Mensagänge. Auch dem technischen Personal in den Werkstätten und am Beschleuniger sowie den Angestellten im Sekretariat gilt mein aufrechter Dank. Ohne die schnelle und unkomplizierte Hilfe wäre vieles nicht möglich gewesen.

Schließlich möchte ich mich bei meinen Eltern bedanken, die immer mit ganzem Herzen unterstützt haben, was ich angefangen habe.

Mein größter Dank gilt meiner Frau Ina für ihre Liebe und Unterstützung. 


\title{
Curriculum Vitae
}

\author{
Persönliche Daten:
}

Name Daniel Stichtenoth

Geburtstag 1. Juni 1979

Geburtsort Göttingen, Deutschland

Nationalität deutsch

Familienstand verheiratet

\section{Bildungsweg:}

$\begin{array}{ll}\text { 1985-1989 } & \text { Grundschule Scheden } \\ \mathbf{1 9 8 9 - 1 9 9 1} & \text { Orientierungsstufe Dransfeld } \\ \mathbf{1 9 9 1 - 1 9 9 8} & \text { Grotefend-Gymnasium, Hann Münden (Abitur) } \\ \mathbf{1 9 9 9 - 2 0 0 5} & \text { Studium der Physik an der Universität Göttingen } \\ & \text { Vordiplom 2001 } \\ & \text { (Note: gut) } \\ & \text { Diplom November 2005 ("Wachstum und Modifikation } \\ & \text { von Halbleiter Nanodrähten") } \\ & \text { (Note: sehr gut) } \\ & \text { Wissenschaftlicher Angestellter, 2. Physikalisches Institut, } \\ \text { 2005-2008 } & \text { Universität Göttingen }\end{array}$

